Self-Assembled and Responsive Supramolecular Nanoparticles

Mediated by Cucurbit[8]uril Host-

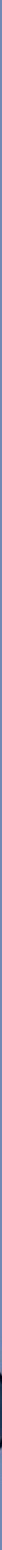

Carmen Stoffelen 


\section{Self-Assembled and Responsive Supramolecular Nanoparticles Mediated by Cucurbit[8]uril Host-Guest Interactions}

Carmen Stoffelen 


\section{Members of the committee:}

Chairman: Prof. dr. ir. J.W.M. Hilgenkamp

Promotor: Prof. dr. ir. J. Huskens

Members: Prof. dr. ir. P. Jonkheijm

Prof. dr. J.F.J. Engbersen

Prof. dr. B.J. Ravoo

Prof. dr. C.A. Schalley

dr. S. Le Gac
(University of Twente)

(University of Twente)

(University of Twente)

(University of Twente)

(Westfälische Wilhelms-Universität Münster)

(Frei Universität Berlin)

(University of Twente)

The research described in this thesis was performed within the laboratories of the Molecular Nanofabrication (MnF) group, the MESA ${ }^{+}$Institute for Nanotechnology and the Department of Science and Technology of the University of Twente. This research was supported by the Council for Chemical Sciences of the Netherlands Organization for Scientific Research (NWO-CW, Vici grant 700.58.443).

Self-Assembled and Responsive Supramolecular Nanoparticles Mediated by Cucurbit[8]uril Host-Guest Interactions

Copyright (c) 2015, Carmen Stoffelen-Janssen, Enschede, The Netherlands

All rights preserved. No part of this work may be reproduced or transmitted in any form, by any means, electronic or mechanical without prior written permission of the author.

ISBN: $\quad 978-90-365-3849-7$

DOI: $\quad 10.3990 / 1.9789036538497$

Cover art: Jan-Stefan Janssen and Carmen Stoffelen

Printed by: Gildeprint Drukkerijen - the Netherlands 


\title{
SELF-ASSEMBLED AND RESPONSIVE SUPRAMOLECULAR NANOPARTICLES MEDIATED BY CUCURBIT[8]URIL HOST-GUEST INTERACTIONS
}

\section{DISSERTATION}

\author{
to obtain \\ the degree of doctor at the University of Twente \\ on the authority of the rector magnificus, \\ Prof. dr. H. Brinksma,
}

on account of the decision of the graduation committee,

to be publicly defended

on Thursday March 26, 2015 at 14.45 h

by

Carmen Stoffelen

Born on June 10, 1983

In Kleve, Germany 
This dissertation has been approved by:

Promotor: $\quad$ Prof. dr. ir. J. Huskens 
"Try to become not a man of success, but try rather to become a man of value."

Albert Einstein

This thesis is dedicated to my family. 



\section{Table of Contents}

Chapter 1: General Introduction 1

1.1 References 3

Chapter 2: Supramolecular Nanoparticles

2.1 Introduction 5

2.2 Self-assembly and non-covalent interactions 6

2.3 Supramolecular nanoparticles by basic non-covalent interactions

2.3.1 Supramolecular nanoparticles stabilized by hydrophobic 9 interactions

2.3.2 Supramolecular nanoparticles stabilized by hydrogen bonding

2.3.3 Supramolecular nanoparticles stabilized by electrostatic interactions

2.4 Supramolecular nanoparticles mediated by host-guest interactions

2.4.1 Cyclodextrin-based supramolecular nanoparticles

2.4.1.1 Monovalent cyclodextrin-stabilized SNPS

2.4.1.2 Multivalent cyclodextrin-stabilized SNPS

2.4.1.3 Cyclodextrin supramolecular nanoparticles stabilized by hydrophobic interactions

2.4.1.4 Cyclodextrin supramolecular nanoparticles stabilized by electrostatic interactions

2.4.2 Calix[n]arene and pillar[n]arene supramolecular nanoparticles

2.4.2.1 Multivalent calix[n]arene/pillar[n]arene-stabilized supramolecular nanoparticles

2.4.2.2 Calix[n]arene and pillar[n]arene supramolecular nanoparticles stabilized by hydrophobic interactions 
2.4.2.3 Calix[n]arene supramolecular nanoparticles stabilized

by electrostatic interactions

2.4.3 Cucurbit[n]uril supramolecular nanoparticles 34

2.4.3.1 Multivalent cucurbit[n]uril-stabilized supramolecular $\quad 34$ nanoparticles

2.4.3.2 Cucurbit[n]uril supramolecular nanoparticles

stabilized by hydrophobic interactions

2.4.3.3 Cucurbit[n]uril supramolecular nanoparticles

stabilized by electrostatic interactions

2.5 Conclusions

Chapter 3: Size-Tunable Supramolecular Nanoparticles Mediated by Ternary Cucurbit[8]uril Host-Guest Interactions

3.1 Introduction

3.2 Results and Discussion

3.2.1 Synthesis of the supramolecular building blocks 50

3.2.2 Supramolecular nanoparticle formation in aqueous solution $\quad 50$

3.2.3 Size tunability of supramolecular nanoparticles 53

3.3 Conclusions $\quad 55$

3.4 Acknowledgements $\quad 55$

3.5 Experimental Section $\quad 55$

3.5.1 Materials 55

3.5.2 Synthetic procedures $\quad 55$

3.5.3 Methods 57

3.5.4 Equipment 58

$\begin{array}{ll}3.6 \text { References } & 59\end{array}$

Chapter 4: Effects of the Molecular Weight and the Valency of 61 Guest-modified Poly(ethylene glycol)s on the Stability, Size and Dynamics of Supramolecular Nanoparticles

4.1 Introduction 62

4.2 Results and Discussion 63 
4.2.1 SNP formation using different shell components

4.2.2 SNP formation with monovalent Np-PEG of varying length 66

4.2.3 Supramolecular nanoparticle formation with stoppers of 70

different valencies

$\begin{array}{ll}4.3 \text { Conclusions } & 74\end{array}$

4.4 Acknowledgements $\quad 75$

4.5 Experimental Section $\quad 75$

4.5.1 Materials $\quad 75$

4.5.2 Synthetic procedures $\quad 75$

$\begin{array}{ll}4.5 .3 \text { Methods } & 79\end{array}$

$\begin{array}{ll}\text { 4.5.4 Equipment } & 79\end{array}$

4.6 References $\quad 80$

Chapter 5: Dual Stimuli-Responsive Self-Assembled 83 Supramolecular Nanoparticles

5.1 Introduction $\quad 84$

5.2 Results and Discussion $\quad 86$

5.2.1 Supramolecular nanoparticle formation in aqueous solution $\quad 86$

5.2.2 Dual responsive supramolecular nanoparticle disassembly 88

$\begin{array}{ll}5.3 \text { Conclusions } & 94\end{array}$

5.4 Acknowledgements $\quad 94$

5.5 Experimental Section $\quad 94$

5.5.1 Materials 94

5.5.2 Synthetic procedures 94

5.5.3 Methods 96

5.5.4 Equipment 98

$\begin{array}{ll}5.6 \text { References } & 99\end{array}$

Chapter 6: Programmed Disassembly of Supramolecular $\quad 101$ Nanoparticles Stabilized by Heteroternary CB[8] Host-Guest Interactions

$\begin{array}{ll}6.1 \text { Introduction } & 102\end{array}$

6.2 Results and Discussion 103

6.2.1 SNP toolbox approach for programmable particle disassembly 103 
6.2.2 SNPs with a photoswitchable shell 105

6.2.3 SNPs with a photoswitchable core 108

6.3 Conclusions $\quad 110$

6.4 Acknowledgements 111

6.5 Experimental Section $\quad 111$

6.5.1 Materials 111

6.5.2 Methods 111

6.5.3 Equipment 112

6.6 References 112

Chapter 7: Zwitterionic Supramolecular Nanoparticles: Self- $\quad 115$ Assembly and Responsive Properties

7.1 Introduction 116

$\begin{array}{ll}7.2 \text { Results and Discussion } & 118\end{array}$

7.2.1 Supramolecular nanoparticle formation in different media $\quad 118$

7.2.2 Responsive properties of zwitterionic supramolecular 123 nanoparticles

$\begin{array}{ll}7.3 \text { Conclusions } & 126\end{array}$

7.4 Acknowledgements $\quad 127$

7.5 Experimental Section 127

7.5.1 Materials 127

7.5.2 Synthetic procedures 127

7.5.3 Methods 128

7.5.4 Equipment 130

$\begin{array}{ll}7.6 \text { References } & 131\end{array}$

Chapter 8: Self-Assembly of Size-Tunable Supramolecular 133 Nanoparticle Clusters in a Microfluidic Channel

8.1 Introduction 134

8.2 Results and Discussion 136

8.2.1 Supramolecular building blocks for SNPC formation 136

8.2.2 Formation of SNPCs in bulk 138

8.2.3 Formation of SNPCs in a microreactor 139

8.2.4 Size tuning of SNPCs by varying the stoichiometry 141 
8.2.5 Size tuning of the SNPCs by varying the residence time 144

8.2.6 Size tuning of the SNPCs by varying the concentrations of the $\quad 147$ building blocks

$\begin{array}{ll}8.3 \text { Conclusions } & 149\end{array}$

8.4 Acknowledgements 150

8.5 Experimental Section $\quad 150$

8.5.1 Materials 150

8.5.2 Synthetic procedures $\quad 150$

8.5.3 Self-assembly procedures 151

8.5.4 Equipment 153

$\begin{array}{ll}8.6 \text { References } & 154\end{array}$

$\begin{array}{ll}\text { Summary } & 157\end{array}$

$\begin{array}{ll}\text { Samenvatting } & 159\end{array}$

$\begin{array}{ll}\text { Acknowledgements } & 161\end{array}$ 


\section{Chapter 1}

\section{General Introduction}

Nanotechnology is an interdisciplinary field that describes the manipulation of objects in small dimensions to provide material properties that are significantly different from those observed in the bulk..$^{1-3}$ In particular, nanoparticles (NPS) of different compositions and designs have attracted much attention in science and society. Despite the concern about their potential toxicity, their new materials properties make them of high interest in electronics, catalysis, textile industry, renewable energy, and health. ${ }^{4-6}$ In particular the application of nanoparticles in biomedicine has provided new strategies for diagnosis and drug delivery. ${ }^{7-9}$

Supramolecular chemistry has gained in importance in chemistry and other disciplines over the past two decades. ${ }^{10}$ The self-assembly of small building blocks by non-covalent interactions, i.e. the process by which individual building blocks organize into ordered structures spontaneously, is the key principle in this area. ${ }^{11}$ Inspired by nature, supramolecular chemistry enables the formation of functional materials in different dimensions from molecular building blocks, based on a toolbox of different non-covalent interactions with variable binding strengths and dynamics. ${ }^{12,13}$ The combination of multiple non-covalent interactions, called multivalency, leads to very stable assemblies in the absence of covalent bonds. ${ }^{14}$ At the same time, the non-covalent origin of the interactions, and its inherent reversibility, enables the design of responsive supramolecular materials.

Supramolecular nanoparticles (SNPs) are formed by multiple building blocks that are brought together by multiple non-covalent supramolecular interactions. SNPs can be divided into two major classes. Soft SNPs employ only organic and/or polymeric building blocks and are used, for example, for drug or gene delivery applications. ${ }^{15,16}$ Alternatively, 
metallic or inorganic nanoparticles, modified with supramolecular binding motifs at the exterior, can be used as hard building blocks in SNPs and can, for example, be used as detection tool in various screening essays ${ }^{17,18}$ or for biomedical applications. ${ }^{19}$

The research described in this thesis aims to understand the design rules of SNPs, their self-assembly and triggered disassembly, and the dynamics of these processes. Therefore the heteroternary complexes of cucurbit[8]uril, methyl viologen and naphthol $(\mathrm{CB}[8] / \mathrm{MV} / \mathrm{Np})$ and of $\mathrm{CB}[8], \mathrm{MV}$ and azobenzene (CB[8]/MV/Azo) have been used for the formation of soft SNPs (Chapters 3-7) and for the clustering of hard SNPs employing MV-functionalized silica NPs as a building block (Chapter 8). Control over the SNP size, the assembly kinetics, and the disassembly pathway are major objectives of the work presented here.

Chapter 2 provides a literature overview of soft supramolecular nanoparticles. Emphasis is placed on the nature of the underlying supramolecular interaction, the valency of the stabilizing interactions and on the potential biomedical applications.

In Chapter 3, a novel strategy for the formation of SNPs is reported based on multiple $\mathrm{CB}[8] / \mathrm{MV} / \mathrm{Np}$ charge transfer complexes, thus employing $\mathrm{CB}[8]$ as a host instead of the typically used cyclodextrin. The self-assembly of the supramolecular building blocks into SNPs in aqueous solution and the size tuning of the SNPs by the stoichiometry of the components are studied. The dynamics of the assembly is investigated as well and is related to the dynamics of the individual ternary complex.

The same SNP formation strategy has been used in Chapter 4 to investigate the role of the guest-modified hydrophilic polymer assembling in the shell of the SNPs thus functioning as a stopper. Therefore naphthol was grafted onto oligo and poly(ethylene glycol)s with different lengths and SNP self-assembly was carried out. The colloidal stability and the size tuning are described, as well as the influence of the valency of the stopper on the SNP morphology and dynamics.

In Chapter 5, dual stimuli-responsive SNPs are presented, which are stabilized by multiple $\mathrm{CB}[8] / \mathrm{MV} /$ Azo complexes. Photo-induced trans-cis isomerization of the Azo and chemical reduction of MV leads to disrupture of the heteroternary complex going and subsequent SNP disassembly. By monitoring the particle size in time, the dynamics of the SNP disintegration is studied in detail.

SNP formation by a toolbox approach is described in Chapter 6. Hereto, SNPs are assembled by ternary $\mathrm{CB}[8] / \mathrm{MV} / \mathrm{Np}$ or $\mathrm{CB}[8] / \mathrm{MV} / \mathrm{Azo}$ interactions exclusively, or by a combination of the two. Photoisomerization of Azo enables disassembly of the $\mathrm{CB}[8] / \mathrm{MV} / \mathrm{Azo}$ moieties. Thereby, the SNP disassembly is studied as a function of the placement of the CB[8]/MV/Azo moieties in the SNP core, shell, or both. 
The formation and responsive character of zwitterionic SNPs is reported in Chapter 7. Based on the supramolecular CB[8]/MV/Azo interactions, SNPs are formed by assembling a relatively short, non-fouling, Azo-functionalized zwitterionic molecule in the SNP shell. The colloidal stabilities of the zwitterionic SNPs in water, in phosphate-buffered saline at different salt concentrations, and at relatively high protein concentrations have been studied in detail. The effect of $\mathrm{pH}$ on the particle morphology was monitored as well.

Finally, the supramolecular assembly of MV-functionalized silica nanoparticles by Npterminated building blocks in the presence of $\mathrm{CB}[8]$ is demonstrated in Chapter 8 . Therein the self-assembly and size control of supramolecular NP clusters is compared for SNP preparation in bulk and in a microfluidic device. The effect of the controlled diffusion time of the supramolecular components in the microfluidic device, the stoichiometry of the host and guest binding partners, and the competition of the monovalent and multivalent Np-bearing building blocks on the size of the supramolecular NP clusters has been studied in detail.

\subsection{References}

1. C. N. R. Rao, G. U. Kulkarni, P. J. Thomas and P. P. Edwards, Chemistry - A European Journal, 2002, 8, 28-35.

2. G. M. Whitesides, Small, 2005, 1, 172-179.

3. F. Sanchez and K. Sobolev, Construction and Building Materials, 2010, 24, 2060-2071.

4. G. Oberdörster, V. Stone and K. Donaldson, Nanotoxicology, 2007, 1, 2-25.

5. H. Bouwmeester, S. Dekkers, M. Y. Noordam, W. I. Hagens, A. S. Bulder, C. de Heer, S. E. C. G. ten Voorde, S. W. P. Wijnhoven, H. J. P. Marvin and A. J. A. M. Sips, Regulatory Toxicology and Pharmacology, 2009, 53, 52-62.

6. A. N. Shipway, E. Katz and I. Willner, ChemPhysChem, 2000, 1, 18-52.

7. N. L. Rosi and C. A. Mirkin, Chemical reviews, 2005, 105, 1547-1562.

8. K. S. Soppimath, T. M. Aminabhavi, A. R. Kulkarni and W. E. Rudzinski, Journal of Controlled Release, 2001, 70, 1-20.

9. G. Y. Tonga, K. Saha and V. M. Rotello, Advanced Materials, 2014, 26, 359-370.

10. G. M. Whitesides and B. Grzybowski, Science, 2002, 295, 2418-2421.

11. J.-M. Lehn, Proceedings of the National Academy of Sciences, 2002, 99, 4763-4768.

12. O. Ikkala and G. ten Brinke, Science, 2002, 295, 2407-2409.

13. T. Aida, E. W. Meijer and S. I. Stupp, Science, 2012, 335, 813-817.

14. A. Mulder, J. Huskens and D. N. Reinhoudt, Organic \& Biomolecular Chemistry, 2004, 2, 3409-3424.

15. K.-J. Chen, M. A. Garcia, H. Wang and H.-R. Tseng, in Supramolecular Chemistry, John Wiley \& Sons, Ltd, 2012.

16. Q.-D. Hu, G.-P. Tang and P. K. Chu, Accounts of Chemical Research, 2014, 47, 20172025.

17. K. Saha, S. S. Agasti, C. Kim, X. Li and V. M. Rotello, Chemical Reviews, 2012, 112, 2739-2779.

18. M.-C. Daniel and D. Astruc, Chemical Reviews, 2004, 104, 293-346. 
19. Z. Li, J. C. Barnes, A. Bosoy, J. F. Stoddart and J. I. Zink, Chemical Society Reviews, 2012, 41, 2590-2605. 


\section{Chapter 2}

\section{Supramolecular Nanoparticles}

Supramolecular chemistry provides a tool for the formation of highly ordered structures by means of non-covalent interactions. Soft supramolecular nanoparticles are selfassembled nanoassemblies, based on small building blocks and stabilized by basic noncovalent interactions, selective host-guest interactions or a combination of different interaction types. This chapter gives an overview of the existing approaches for the formation of supramolecular nanoparticles. The approaches are ordered based on the nature of the stabilizing supramolecular interaction while focusing on the aspects that determine the particle structure. When applicable, the use of these self-assembled nanostructures as vectors in molecular diagnostics and therapeutics is described as well. Generally, the stable yet reversible nature of supramolecular interactions and the chemical flexibility offers great prospects for the development of highly engineered nanoparticles which are compatible with the complexity of living systems. 


\subsection{Introduction}

Nanoparticles (NPs) attract heavy interest in nanoscience, owing to their size-specific chemical and physical properties. Next to catalytic, optical, sensor and/or electronic applications, the use of NPs in biomedicine has gained intense scientific interest over the past two decades. ${ }^{1,2}$ Countless studies have been carried out for the implementation of nanoparticles in biologically and pharmaceutically relevant systems. These achievements have been summarized in numerous reviews, which are giving either a general perspective of the field ${ }^{2-5}$ or are focused on a particular application such as drug delivery, ${ }^{6-9}$ imaging technology ${ }^{10,11}$ or theranostics. ${ }^{12-15}$ Recently, the use of NPs as adhesive material for regenerative wound healing purpose has been reported as well. ${ }^{16}$ The combination of supramolecular chemistry and NP technology provides new prospects in the field of NPs for biomedical applications. ${ }^{17}$ The non-covalent origin and accompanying ability for building blocks to assemble and disassemble spontaneously is one of the main advantages that supramolecular chemistry contributes to this synergism.

\subsection{Self-assembly and non-covalent interactions}

Dynamic self-assembly rules biological processes in vivo. ${ }^{18}$ Cell formation, proliferation and replication are based on complex processes such as DNA (deoxyribonucleic acid) replication, mitosis, microtubule growth and shrinking, all in which selective assembly and disassembly are involved. The exceptional feature of non-covalent interactions is their reversibility; binding and unbinding occurs without rupture or alteration of the initial binding partners. Inspired by the control of matter at the smallest scale, nanotechnology in particular uses self-assembly and self-organization of molecules and bigger entities for the fabrication of desired structures and functional materials. ${ }^{18,19}$

Supramolecular chemistry makes use of a toolbox of different non-covalent interactions. These interactions range from relatively weak interactions such as hydrogen bonding, $\pi$ $\Pi$ stacking, Van der Waals forces or hydrophobic interactions to stronger ionic and dipolar bonds. Next to these basic non-covalent interactions, host-guest chemistry provides a powerful paradigm to supramolecular chemistry. Host-guest formation, leading to the inclusion of a guest molecule into a host, is commonly based on a combination of some of these intermolecular forces. The choice of solvent is very important in synthetically self-assembled systems as it has a big impact on the affinity and selectivity of the formed complex. Naturally occurring self-assembled systems and nanometer-sized assemblies designed for biomedical applications are limited to aqueous media. Non-covalent supramolecular motifs capable of self-assembly in water have been the subject of several recent reviews. ${ }^{20-23}$ 
In all self-assembly processes, thermodynamics plays an important role in explaining the stability and properties of the resulting structure. The key challenge in synthetic selfassembly is to control multiple underlying non-covalent interactions to achieve an energetically more favorable state after assembly. This is achieved by the interplay between entropic and enthalpic factors. When zooming in solely on the interaction between a host and a guest, the interactions between these molecules are enthalpically favorable and have to overcome the loss in entropy caused by complexation. ${ }^{19}$ At the same time, entropically favorable solvent release and solvent rearrangement can contribute positively to the energy gain or even drive the formation of the supramolecular assemblies entirely. Contribution by the solvent is particularly important in polar solvents such as water, in which the interaction amongst solvent molecules and between solvent and solute is of comparable strength as the interaction between the binding partners.

As in covalent chemical reactions, the energy levels and the energy barriers determine whether the thermodynamic or a kinetic product is formed during molecular selfassembly. The thermodynamic product is the most stable product with the lowest achievable energy, irrespective of any intervening barrier. If the supramolecular product is in thermodynamic equilibrium, all intermediate states are reversible under the reaction conditions employed. This state ensures that the self-assembly is dynamic and an equilibrium between association and dissociation is present. In contrast, a kinetic product is associated with the lowest energy barrier to its formation so it is the fastest formed intermediate in the self-assembly process. Nature frequently uses such kinetically trapped states, for example in enzymatic catalysis, but developing artificial out-ofequilibrium self-assembled systems is challenging. ${ }^{24}$

Multivalency, the interaction of multivalent receptors and multivalent ligands, plays an important role in biological processes and provides a simple strategy to increase the binding affinities in synthetic self-assembled structures. ${ }^{25,26}$ Multivalent binding events can occur between complementary functionalities on a single building block or between two complementary building blocks and leads to products bound by multiple inter and intramolecular connections. ${ }^{27}$ For instance, single hydrogen bonds or Van der Waals interactionsare relatively weak $(0.4$ to $20 \mathrm{~kJ} / \mathrm{mol})$ and do not differ strongly from the omnipresent thermal energy at room temperature $(2.5 \mathrm{~kJ} / \mathrm{mol})$. Nevertheless, stable supramolecular structures have been reported that are exclusively formed by hydrogen bonding or Van der Waals interactions. ${ }^{28}$ This could only be achieved by organizing the molecules using a multitude of these relatively weak interactions. A prominent example of multivalency in nature is the formation of the DNA double helix. 
The multivalent character of the binding event bestows unique thermodynamic and kinetic characteristics onto the supramolecular complex. ${ }^{29,30}$ Modeling the thermodynamics of non-covalent binding events has shown that the associationdissociation mechanisms of monovalent and multivalent structures differ strongly from each other. ${ }^{31}$ Taking the effective molarity (E.M., i.e. the probability of binding sites to meet depending on conformation length and flexibility $)^{32}$ into consideration, a concentration dependent binding mode has been introduced for multivalent interactions. When the effective molarity of a guest moiety, already bound to the multivalent receptor in a preceding intermolecular binding step, is higher than the concentration of free guest in solution $\left([\mathrm{G}]_{\text {sol }}\right)$, intramolecular, i.e. multivalent, binding is favored (Figure 2.1). However, when $E M$ is lower than $[G]_{\text {sol, }}$ intermolecular connections, i.e. supramolecular polymerization or cross-linking, take over.

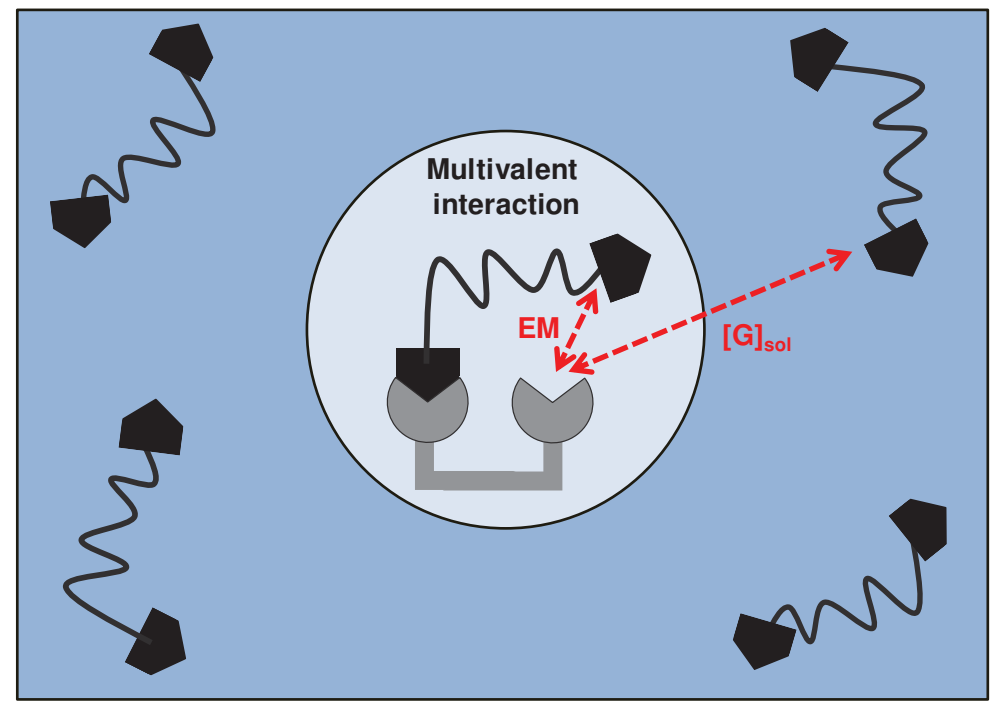

Figure 2.1: Schematic representation of the effective molarity (EM) responsible for favored intramolecular multivalent binding instead of intermolecular binding with guest molecules from solution. ${ }^{29}$

In kinetic terms, the dissociation of supramolecular assemblies connected via multivalent interactions is typically much slower than in assemblies stabilized by monovalent interactions of the same origin. Even though the individual dissociation rate of a single interaction in a multivalently connected supramolecular assembly does not differ from the dissociation rate of the corresponding monovalent interaction, the overall dissociation rate can decrease by orders of magnitude because the probability that all bonds are broken simultaneously dictates the overall dissociation rate. ${ }^{31}$ Thus, the overall dissociation rate of a multivalent supramolecular assembly is determined by the dissociation and concentration of an intermediate in which only one bond remains. As the population of this intermediate is suppressed by rebinding induced by the high effective concentration, extremely low dissociation rates are commonly observed for 
multivalently linked assemblies. The use of multivalency as a chemical organization principle for the fabrication of nanomaterials, scaffold architectures and functional materials was reviewed by $u^{29}$ and, more recently, by Haag et al. ${ }^{33}$

\subsection{Supramolecular nanoparticles by basic non-covalent interactions}

In general, the field of supramolecular NPs (SNPS) can be divided into two different classes. We focus here on soft SNPs which are formed and stabilized by non-covalent interactions of individual molecules. In contrast, hard inorganic or polymeric NPs that are decorated with supramolecular receptors at the shell of the NPs and provide so-called network aggregates, are not covered here. Numerous reviews have appeared which summarize the efforts made for the formation and application of organic-inorganic hybrid materials formed by hard supramolecular nanoparticles. ${ }^{34-37}$ More specifically, Rotello and coworkers have focused on the application of surface-engineered NPs for nanomedicine. . $^{3,38-40}$

\subsubsection{Supramolecular nanoparticles stabilized by hydrophobic interactions}

Hydrophobic interactions constitute the driving force of apolar molecules to aggregate in aqueous solution. In nature, non-covalent hydrophobic interactions are important for protein folding, lipid bilayer formation and the insertion of membrane proteins into bilayers. The formation of such assemblies is driven by entropic and enthalpic gains. In the presence of an apolar molecule, water molecules are bound to the solute but with fewer $\mathrm{H}$-bonds than in the bulk solution. By aggregation of the apolar molecules into higher assemblies, the total interface area between water and solute molecules is reduced and thereby the fraction of bound water molecules decreases, resulting in an overall favorable free energy change.

Scientists have already made use of this hydrophobic effect for a long time, and typically amphiphiles, molecules with hydrophilic and hydrophobic fragments, are used for the formation of distinct structures. In 1913 James W. McBain discovered that the conductivity of a soap solution changes with concentration. In the following century, countless studies have focused on the self-assembly of amphiphiles in aqueous environment. ${ }^{41,42}$ Next to the well-known micelle structure (Figure 2.2), other nanostructures such as liposomes, ${ }^{43,44}$ polymersomes, ${ }^{45,46}$ tubes, $^{47}$ or even helices ${ }^{48}$ can be formed depending on the structure of the amphiphilic molecule. By tuning the amphiphilicity transformation of the formed assemblies can be achieved. ${ }^{49}$ 


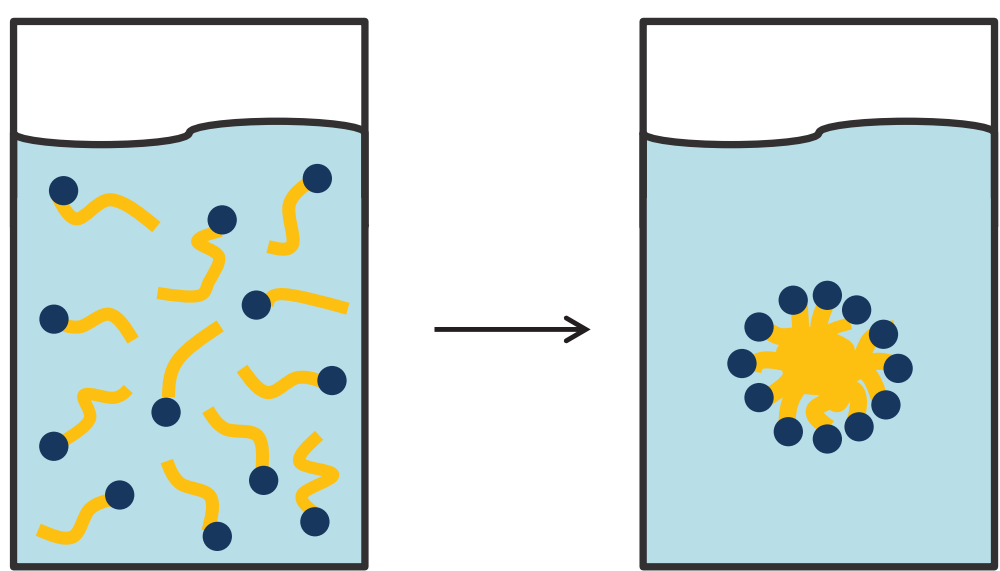

Figure 2.2: Schematic representation of micelle formation in an aqueous environment. The selfassembly of amphiphilic molecules reduces contacting of the hydrophobic segments with water.

\subsubsection{Supramolecular nanoparticles stabilized by hydrogen bonding}

Self-organization of molecular systems via multiple intra- and intermolecular hydrogen bonds is an established strategy for the formation of self-assembled structures. ${ }^{50}$ Taking water molecules as an example, hydrogen bonding is based on the attractive interaction of a hydrogen atom with the electron pair-donating oxygen of another water molecule (Figure 2.3a). This leads to attractive forces that are relatively weak (5 to $30 \mathrm{~kJ} / \mathrm{mol}$ ) and depend on the heteroatoms on either side of the hydrogen atom, but multiplication of these interactions can lead to very stable self-assembled structures, for example, as shown for the formation of rosettes in Figure 2.3c. ${ }^{51}$

a)

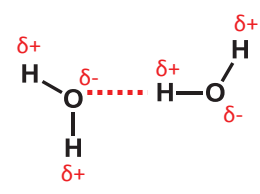

b)<smiles></smiles>

c)

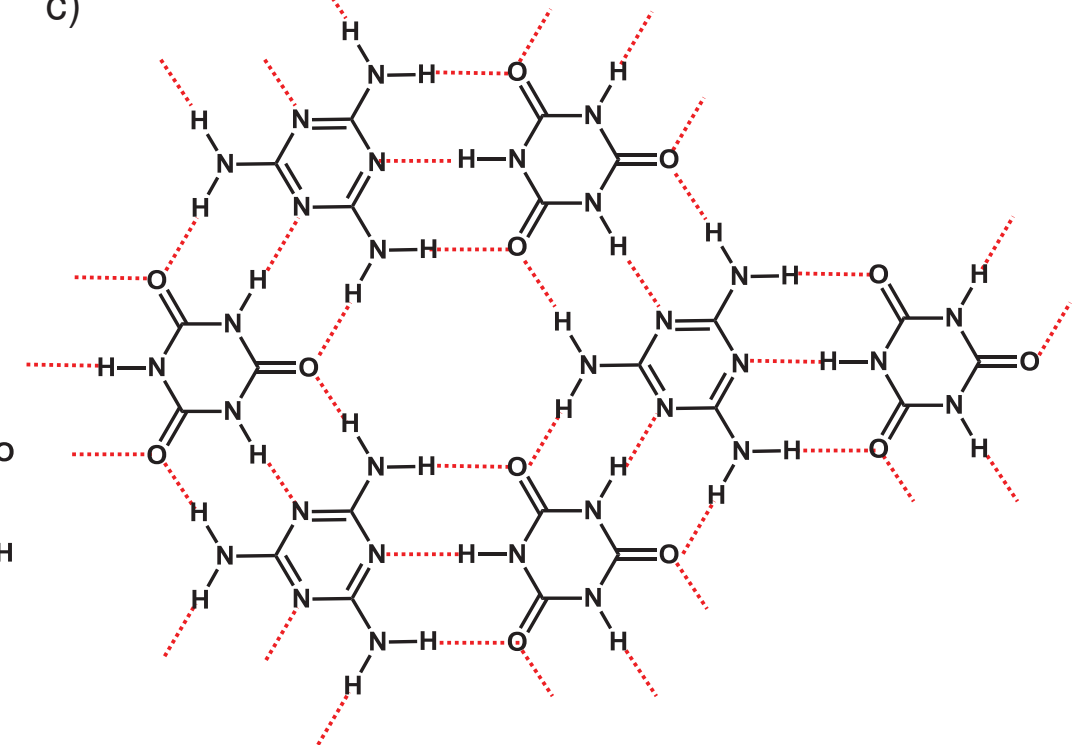

Figure 2.3: a) Model of a single hydrogen bond between two water molecules based on the interaction between hydrogen bond-donating and accepting water molecules. b) Complementary hydrogen bonding motifs of melamine and cyanuric acid. c) Formation of hydrogen-bonded macrocyclic structures called rosettes by complementary melamine and cyanuric acid molecules. 
The use of multiple hydrogen bonding motifs (called dyads) in the formation of stabilized assemblies was applied in NP research for the clustering of dyad-functionalized (hard, inorganic) NPs, ${ }^{52,53}$ micellar structures ${ }^{54,55}$ and the formation of polymeric SNPs. Rotello and coworkers reported the formation of giant supramolecular vesicles by inter-chain hydrogen bonding between a diaminopyridine (DAP)-functionalized polymer and a thymine-based polymer. ${ }^{56} \mathrm{~A}$ comparable type of vesicle structure was formed by the same dyad interaction using DAP and uracil-functionalized poly(norbornene). ${ }^{57}$ Differential interference contrast measurements have shown that the instantaneously formed polymersomes are metastable and fuse into larger vesicles over time. Stable but temperature and competitor responsive microspheres were formed by dissolving DAPfunctionalized poly(styrene) in chloroform at $20^{\circ} \mathrm{C}$ under the formation of a two-fold hydrogen bond. ${ }^{58}$ By heating the microspheres to $50^{\circ} \mathrm{C}$, dissociation was observed which was reversible upon cooling to room temperature. Furthermore, upon addition of thymine-functionalized poly(styrene) these microspheres transformed into vesicles, based on the more stable dyad interactions (Figure 2.4) of the hetero pair formation. This process was found to be reversible as the addition of an excess of DAP polymer reverted the vesicles to microspheres. Interestingly, in contrast to the microsphere structures, uncontrolled aggregation of the dyad-stabilized vesicles occurred upon heating. Another study reported the formation of size-tunable microspheres by mixing PEGylated DAP polymers with a complementary thymine polymer. ${ }^{59}$ The size of the supramolecular structures depended strongly on the polymer length. In this case, an irreversible morphology change was shown by photo-chemical cycloaddition with UV light.

The layer-by-layer (LbL) assembly technique is a powerful strategy for the formation of self-assembled polymeric films and NPs. In the LbL approach, originally developed for polyelectrolytes, ${ }^{60,61}$ films are alternatingly assembled using complementary interactions. Besides films on flat surfaces, hollow supramolecular capsules have been formed by coating solid NPs with polyanions and polycations by LbL assembly based on hydrogen bonding prior to removal of the templating NP. Caruso and coworkers have provided an overview of hydrogen-bonded multilayer films and capsules, and their application in the biomedical field. ${ }^{62}$ 
a)

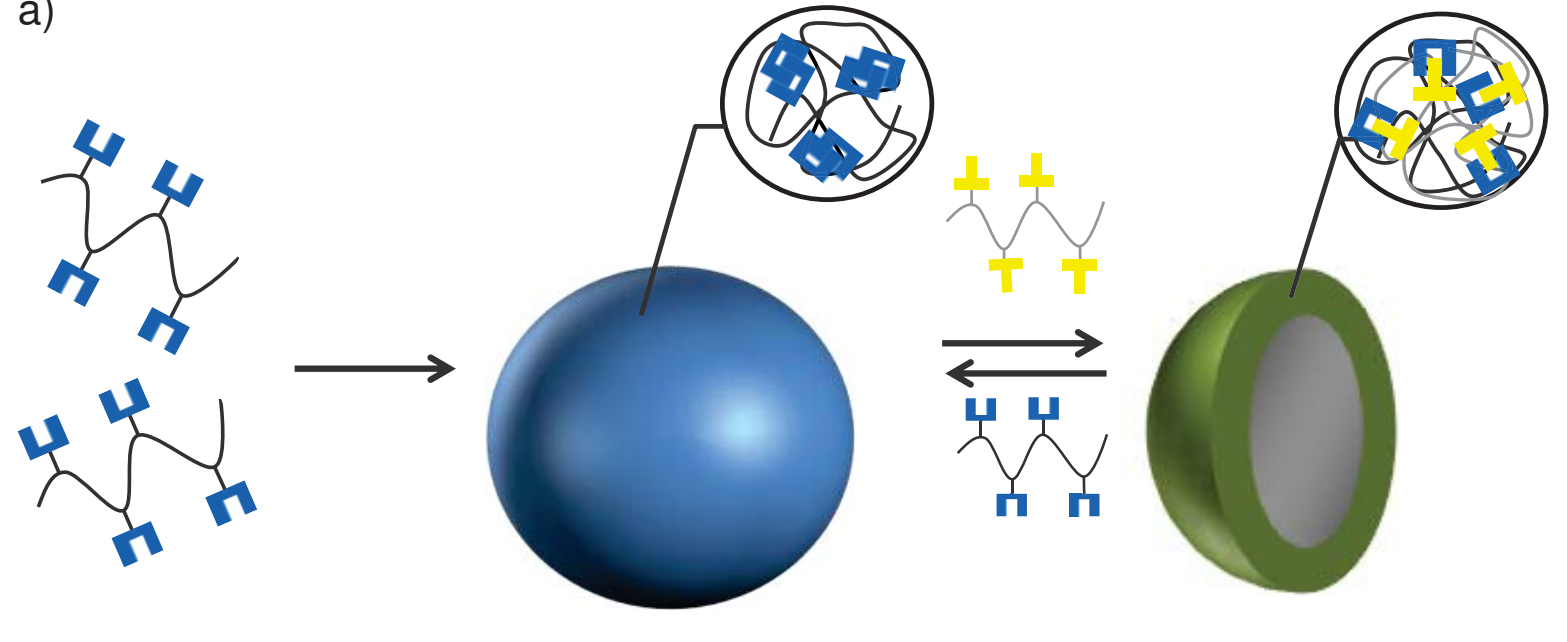

b)<smiles>C=Cc1ccc(COc2ccc(NC(=O)CC)c(NC(=O)CC)c2)cc1C=O</smiles>

$$
=
$$

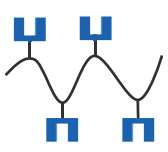

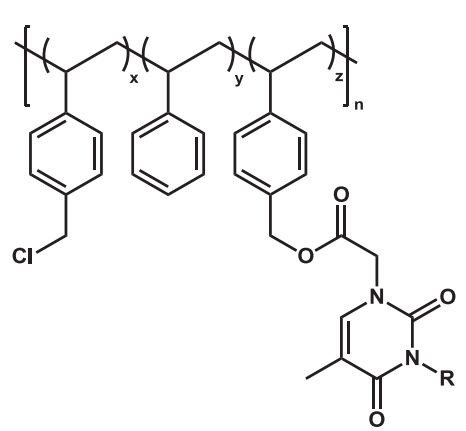

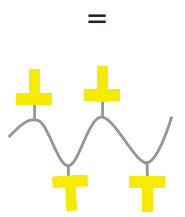

c)

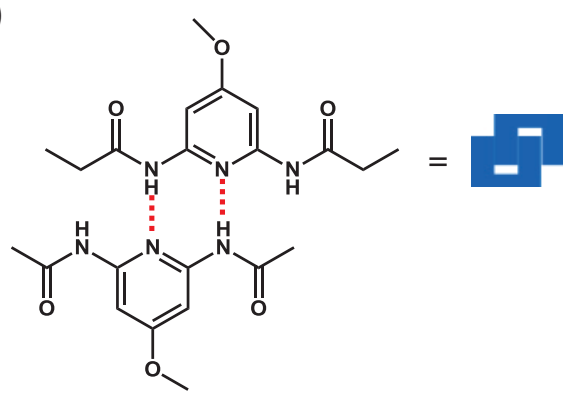

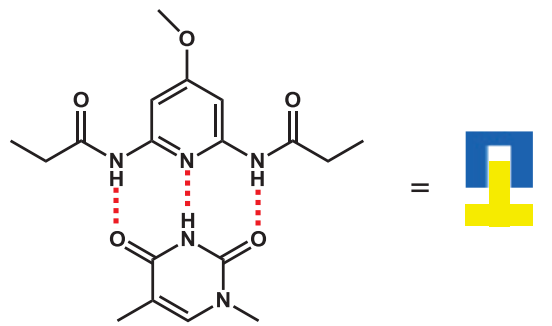

Figure 2.4: a) Schematic illustration of microsphere formation and reversible morphology control from microsphere to vesicle using specific non-covalent interactions. b) Chemical structures of diaminopyridine (DAP) and thymine polymers c) Two-fold hydrogen bonding between two DAP moieties and three-fold hydrogen bonding between DAP and thymine moieties. ${ }^{58}$

\subsubsection{Supramolecular nanoparticles stabilized by electrostatic interactions}

SNPs mediated by electrostatic interactions are based on the attraction of oppositely charged species. Depending on the origin of these building blocks, the individual interaction strength can vary between $5-350 \mathrm{~kJ} / \mathrm{mol}$. The best known approach for the fabrication of SNPs by electrostatic interactions is the formation of non-viral gene delivery systems. These nucleic acid-based therapeutics are formed by the interaction of negatively charged nucleic acids with either cationic lipids forming lipoplexes or cationic polymers forming polyplexes (Figure 2.5). ${ }^{63}$ As these types of SNPs are promising candidates for gene delivery, tremendous efforts have been made within this field, and these have been summarized in several reviews. ${ }^{64-66}$ 
a)
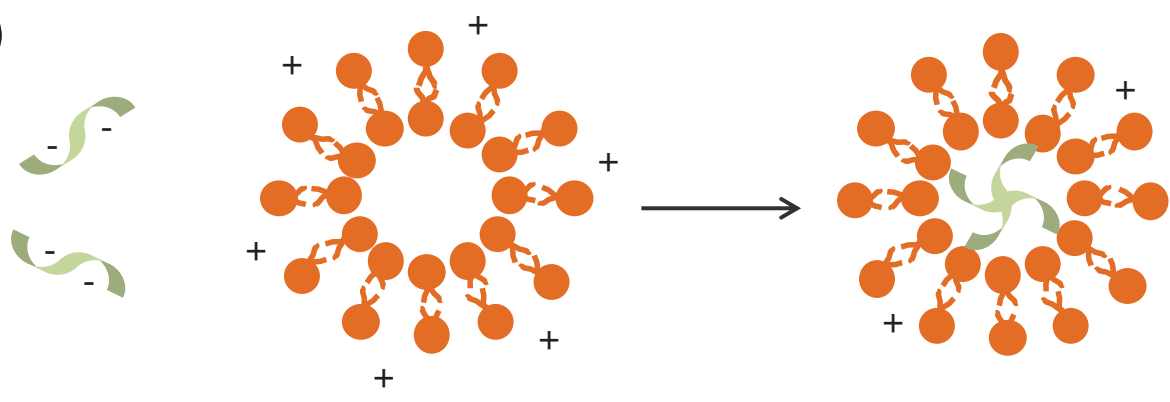

b)
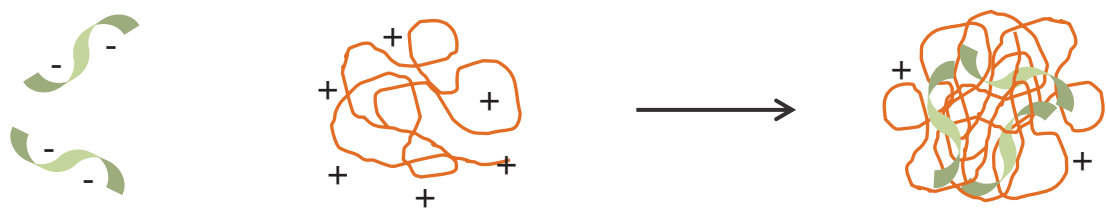

Figure 2.5: Schematic representation of a) lipoplex and b) polyplex formation of negatively charged DNA in the presence of cationic lipids or polymers, respectively.

Chitosan-based polyelectrolyte complexes possess high potential as building material for drug delivery vesicles because of their biocompatibility, biodegradability and low toxicity ${ }^{67}$ In combination with anionic polysaccharides, such as alginate, ${ }^{68}$ carrageenan, ${ }^{69}$ hyaluronic acid, ${ }^{70}$ pectin, ${ }^{71,72}$ xanthan, ${ }^{73,74}$ gum kondagogu ${ }^{75}$ and gum ghatti, ${ }^{76}$ the polycation can form SNPs based exclusively on electrostatic interactions. More specifically, core-shell SNPs resulted from a mixture of chitosan with dextran as the polyanion. ${ }^{77-79}$ Depending on the polymer used in excess, either positively or negatively charged SNPs were formed. The complexed polymer segments accumulate within the core of the particles whereas the excess polymer segments segregate in the shell of the SNPs. This enables selective administration of charges to the particles and ensures colloidal stability. Charge screening for negatively and positively charged particles showed that the stability of all SNPs decreases with the amount of salt added. This clearly proves that both types of SNPs are stabilized by electrostatic interactions. Furthermore, the resulting size of the SNPs could be tuned between 140-240 nm depending on the molecular weight of the constructing polymers. Modification of the chitosan polymer with $N$-(2-hydroxyl)propyl-3-trimethylammonium groups increased the water solubility and the stability of the SNPs at physiological $\mathrm{pH}^{.7}{ }^{7 n}$ Inclusion and release studies of bovine serum albumin (BSA) in chemically modified chitosan-dextran SNPs showed that the SNPs are internalized by cells, and that the release of BSA is $\mathrm{pH}$ dependent.

Electrostatically stabilized SNPs have also been formed by the interaction of two polyelectrolytes, one functionalized with protonated amines and the other with deprotonated carboxylic acids. ${ }^{80}$ SNP formation was visualized by fluorescence resonance energy transfer (FRET) between fluorescein and tetramethyl rhodamine-functionalized 
polymers. By incorporation of the recognition peptide selective for protein kinase $A$ (PKA), the SNPs were used to detect PKA activity. In the presence of PKA, the SNPs were phosphorylated and subsequently dissociated. Thereby the distance between the FRET dye partners increased, and a fluorescence increase of fluorescein isothiocyanate was detected. Another strategy to detect the PKA activity with SNPs uses the same particle recipe, but has incorporated the near-infrared (NIR) fluorophore Cy5.5. ${ }^{81}$ The strong NIR fluorescence intensity of the Cy5.5 dye was quenched upon SNP formation, but was recovered upon SNP disassembly due to the interaction of PKA with the SNPs.

\subsection{Supramolecular nanoparticles mediated by host-guest interactions}

Host-guest chemistry describes the complexation of a guest molecule by a host molecule by intermolecular inclusion. Commonly, the host is a large molecule with convergent binding sites, having a central cavity to enable inclusion complexation. ${ }^{82}$ The guest molecule possesses divergent binding sites and its size can range from small cations or anions to elaborate organic molecules. ${ }^{82}$ Inclusion of the guest molecule into the host can be founded solely on hydrophobic interaction, electrostatic interaction, п-п stacking, hydrogen bonding, Van der Waals interactions or a combination of these.

\subsubsection{Cyclodextrin-based supramolecular nanoparticles}

Cyclodextrins (CDs) are cyclic oligosaccharides linked by a-1,4 glycosidic bonds. The best known examples are a, $\beta$ and $y$-CDs (Figure 2.6), which are composed of 6,7 and 8 glucose units, respectively. The typical cone-shaped structure with the hydrophobic interior enables the inclusion complexation of various hydrophobic guests. Owing to their low toxicity and their excellent biocompatibility, cyclodextrins are the most used hosts for the formation of biomedically relevant assemblies. ${ }^{83-85} \mathrm{~A}$ broad variety of small molecules, ions, proteins or even drugs can be encapsulated into CD cavities. The size of the guest molecule is crucial for the host-guest interactions: too large guests cannot be encapsulated in the interior because of steric hindrance, whereas too small molecules cannot interact efficiently with the cavity of the host. In general, Van der Waals forces between the cyclodextrin cavity and the guest component stabilize encapsulation. However, depending on the guest molecule, other supramolecular interactions such as hydrogen bonds and dipole interactions can be involved. Additional entropically favorable release of water molecules from the $C D$ interior can contribute positively to the relatively strong interactions. 

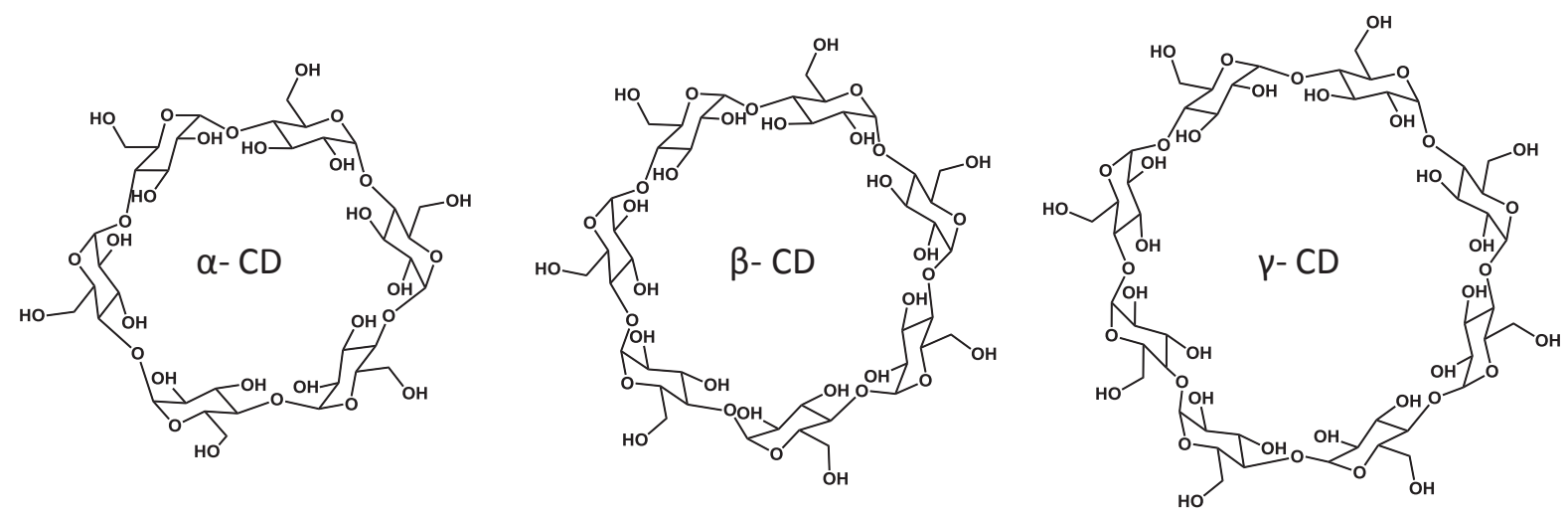

Figure 2.6: Molecular structures of $a$-, $\beta$ - and $y$-cyclodextrin.

\subsubsection{Monovalent cyclodextrin-stabilized SNPs}

Non-viral gene delivery systems, solely stabilized by electrostatic interactions, have been modified with $C D$ to allow for additional stability and functionalization. Davis and coworkers have synthesized linear $\beta$-CD-containing polycations by polymerization of difunctionalized $\beta$-CDs with complementary difunctionalized monomers. ${ }^{86}$ These polymers form polyplexes in the presence of RNA based on electrostatic interactions. Modification of the polymeric structure had strong effects on the polyplex formation, the in vitro transfection efficiency, and the toxicity of the RNA delivery vectors. ${ }^{87-90}$ To overcome the poor stability of these polyplexes under in vitro salt conditions, and to make these gene delivery NPs applicable for therapy, monovalently interacting adamantane end-functionalized poly(ethylene glycol) (Ad-PEG) was added as stabilizer. Upon addition of the hydrophilic Ad-PEG to the preformed polyplex, the Ad moiety interacts with the $\beta$-cyclodextrin cavities, and consequently the Ad-PEG chains assemble at the outside of the polyplexes (Figure 2.7). This surrounding PEG shell leads to stabilization of the particles, decrease of aggregation at physiological ionic strength, ${ }^{91}$ and an increase in cellular uptake of the gene delivery vehicles. ${ }^{92}$

Next to steric stabilization, the interaction of Ad-PEG to the $\beta$-CD-containing polyplex can be used to introduce cell targeting moieties at the shell of the SNPs (Figure 2.7). In general, the NP PEGylation decreases the interaction of the NP surface with the cell. Therefore, targeting moieties are used to bind the delivery vehicles selectively to cell surface receptors and with Ad at one side of the polymer backbone and with galactose, a moiety targeting mediate endocytosis. To introduce targeting moieties, the PEG chains were functionalized with heptacytes, at the other side. ${ }^{91}$ This concept was further expanded to transferrin (Tf), ${ }^{93,94}$ a glycoprotein targeting cancer cells with overexpressed Tf receptors. Generally, the composition of the shell can be tuned by the ratio of the two different stoppers (i.e. with and without targeting moiety). In the case of Tf targeting, 
however, the content of Ad-PEG-Tf was not allowed to exceed $5 \mathrm{~mol} \%$, as intermolecular Tf-Tf interactions led to particle aggregation and destabilization.

a)
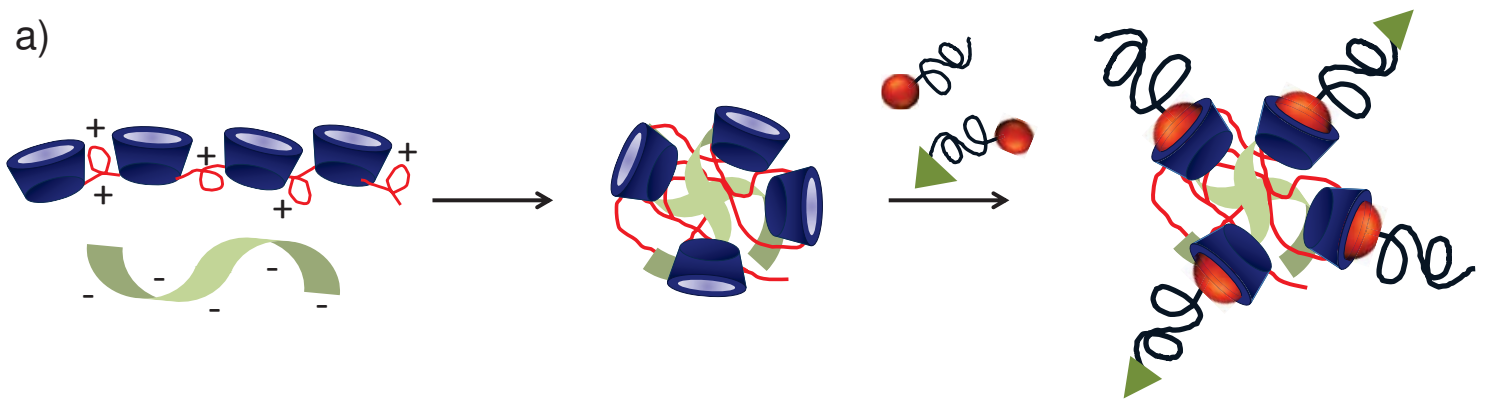

b)
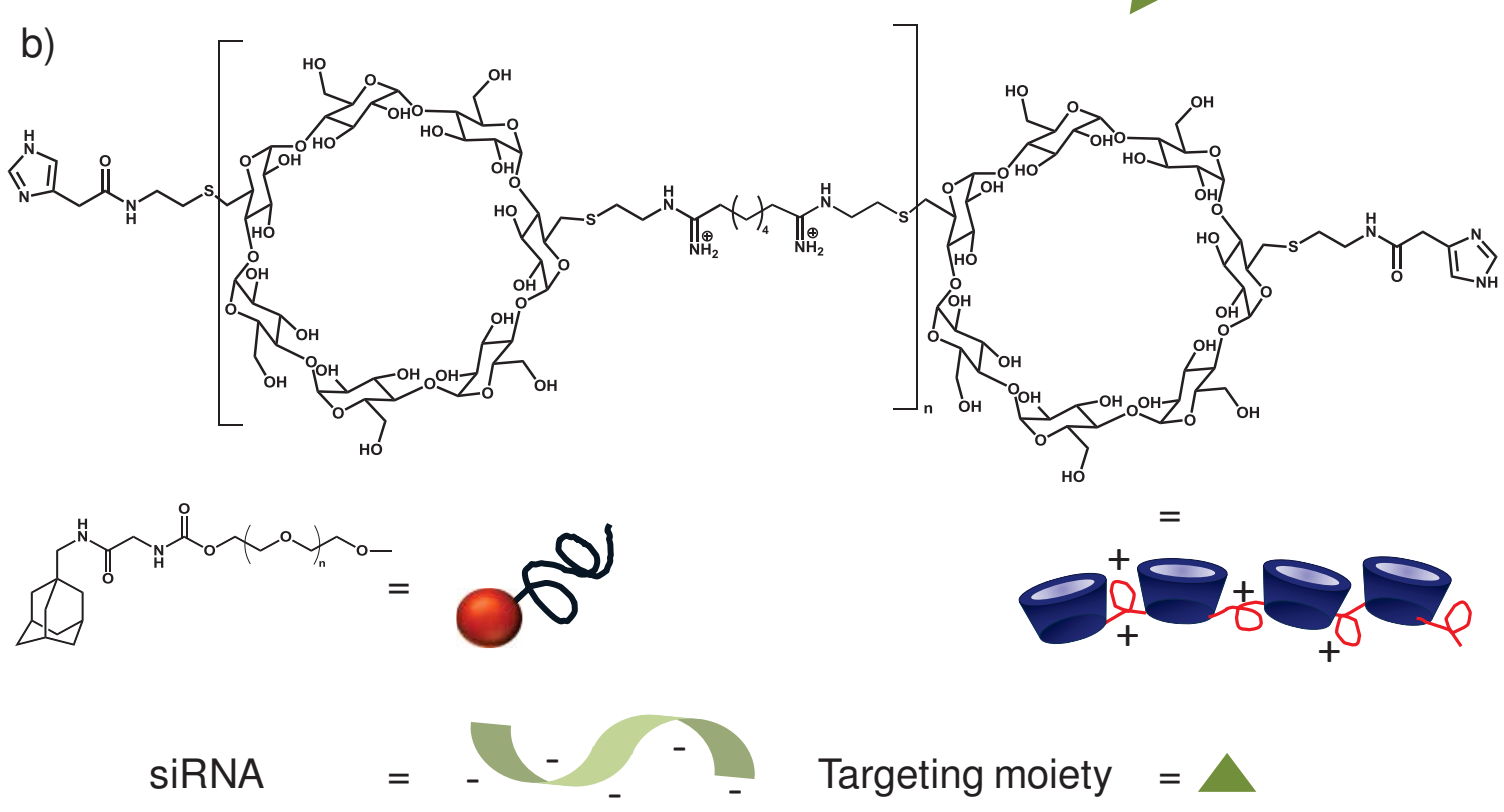

Figure 2.7: a) SNP formation by polyplex formation followed by addition of Ad-PEG and Ad-PEG modified with a cell targeting moiety. b) Chemical structures involved in SNP formation. ${ }^{95}$

In vitro and in vivo studies have been carried out to evaluate the therapeutic performance of these SNP gene delivery vesicles. Modification of the cationic polymer with imidazole groups resulted in a higher in vitro cell transfection efficiency. ${ }^{96}$ Prior to clinical testing in humans, animal studies were carried out in mice ${ }^{97}$ and non-human primates. ${ }^{98}$ Therefore the SNPs were loaded with $\mathrm{Gd}^{3+}$ to evaluate the biodistribution within the animal. Comparative studies with SNPs containing only Ad-PEG or a mixture of Ad-PEG and Ad-PEG-Tf showed that the targeting moiety did not change the biodistribution of the particles in mice but increased the cellular uptake in tumor cells markedly. ${ }^{97}$ Clinical trials, carried out in humans having solid cancer, showed the dosedependent accumulation of SNPs in the tumor tissue which indicates that gene inhibition could be initiated. ${ }^{99}$ Despite the non-immunogenic character of the Ad-PEG-stabilized gene delivery vehicles, studies have shown that the SNPs are rapidly eliminated from circulation. Contrary to normal polyplexes, these SNPs do not disassemble during blood circulation, but the particles accumulate next to the tumor tissue within the glomerular 
basement membrane (GMP), a part of the in vivo renal filtration barrier. ${ }^{100}$ Therein, the positively charged particles are attached to the negative glomerular membrane by electrostatic interactions and SNP disassembly is presumed.

Li and coworkers have reported a similar type of SNPs, in which the monovalent Ad-PEG is used to stabilize and selectively destabilize the DNA and siRNA delivery vehicle. The initial approach uses $\beta$-CD-functionalized chitosan to complex DNA and siRNA electrostatically and form stable gene-containing SNPs in the presence of Ad-PEG. ${ }^{101}$ Modification of this system enabled directed polyplex destabilization in certain cells. ${ }^{102}$ Therefore, SNPs were formed by electrostatic and host-guest interactions of $\beta-C D$ poly(ethylene imine) (CD-PEI), DNA, and a modified type of Ad-PEG. By including an acid-labile disulfide linkage between the host-binding Ad and the shielding PEG polymer, providing Ad-SS-PEG, significant enhancement of in vitro and in vivo gene expression was observed. The disulfide bonds break at lower $\mathrm{pH}$ values, usually observed within tumor cells. The Ad-SS-PEG provides sufficient stabilization of the polyplexes against extracellular salt or BSA-induced aggregation.

A different strategy for the formation of gene delivery vectors was reported by Thompson and coworkers. As seen in Figure 2.8, polyplexes were formed by Ad-modified poly(vinyl alcohol)-PEG (Ad-PVA-PEG), ${ }^{103}$ monovalent cationic $\beta-C D$, and DNA using two different pathways. ${ }^{104,105}$ Either first host-guest interaction of the Ad-PVA-PEG with amino- $\beta-C D$ followed by inclusion of the DNA, or precomplexation of DNA with $C D$ followed by host-guest interaction with the guest polymer led to stable SNPs in solution. The acid-labile character of the benzylidene acetal-linked Ad groups on the PVA backbone enabled SNP degradation and DNA release in the acidic environment of the targeted cancer cells. By functionalization of the guest polymer with a FRET receptor and labeling of the DNA with a FRET donor, SNP assembly and disassembly could be monitored. ${ }^{106}$ The results showed that the SNPs based on the highest host-guest binding affinities had the lowest polydispersity, higher stability, higher cellular uptake and higher transfection efficiency. The preparation method of such non-toxic DNA delivery vectors was further optimized using microfluidic-assisted assembly. ${ }^{107}$ Thereby polyplexes were formed using single $\beta$-CD-modified poly(ethylene imine) (CD-PEI), Ad-conjugated hyaluronic acid (Ad-HA) and DNA. Contrary to bulk mixing, the microfluidic assembly of the SNPs showed a higher reproducibility and enabled flow-rate dependent fine-tuning of the SNP characteristics. This revealed that the SNP formation was not in thermodynamic equilibrium and the microfluidic technique enabled the formation of well-defined, kinetically trapped structures. 
a)

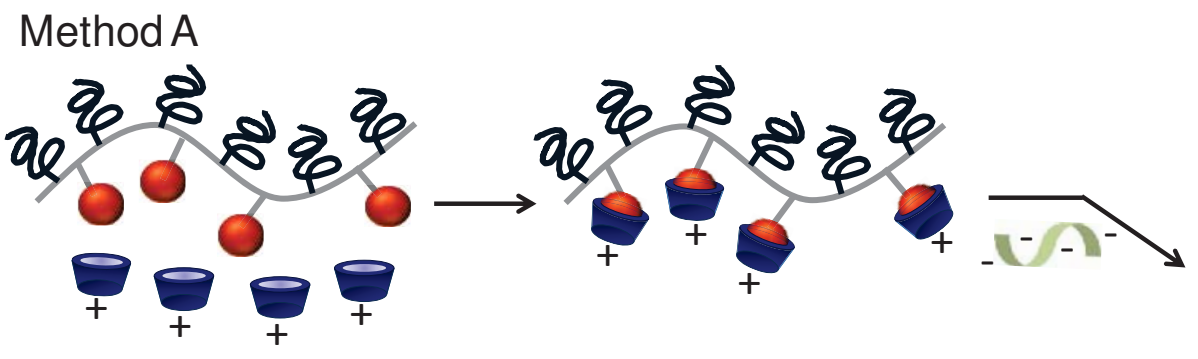

Method B

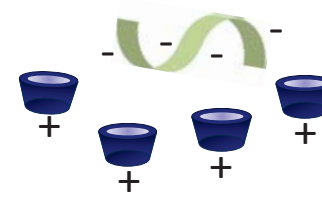
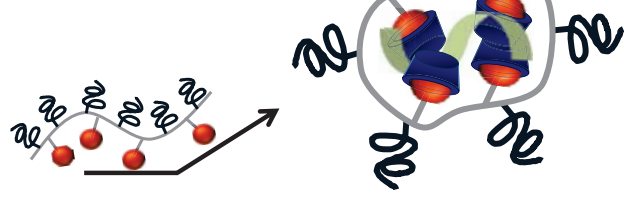

b)
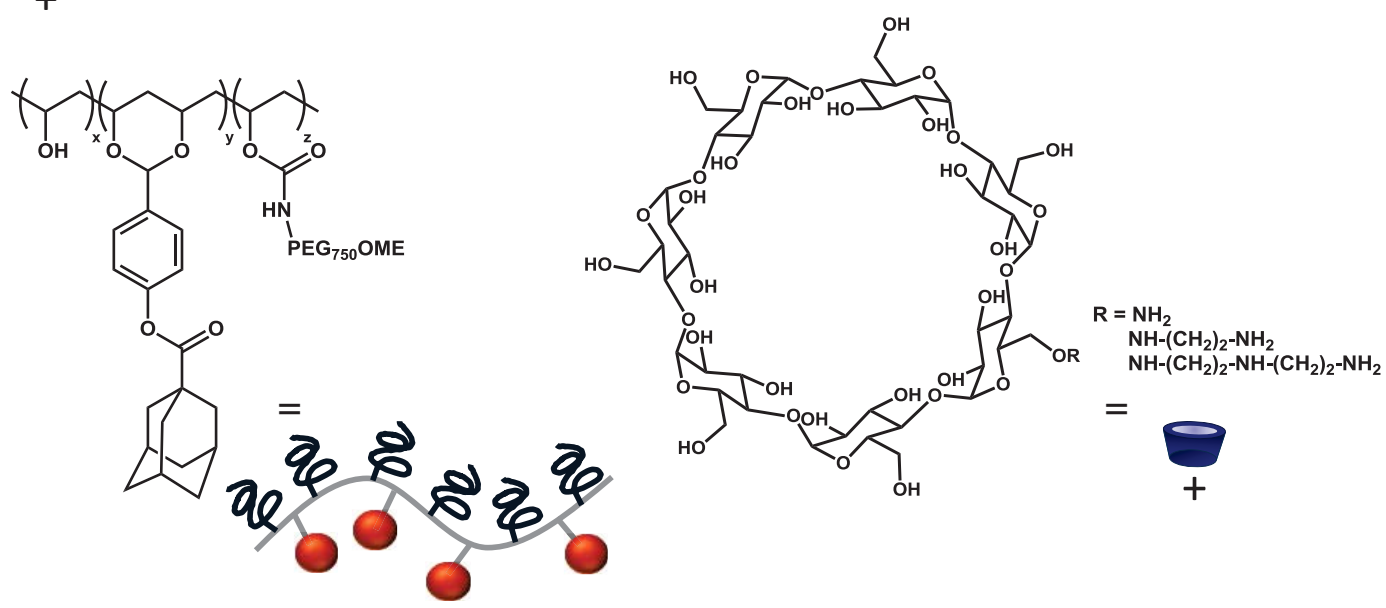

Figure 2.8:a) Formation of a gene delivery vector based on two different formation methods. In method $A$, amino- $\beta-C D$ interacts first with multivalent Ad-functionalized PVA-PEG followed by siRNA condensation. In method $B$, first siRNA condensates with amino- $\beta-C D$ followed by supramolecular interaction with the Ad-bearing polymer. b) Structure of Ad-modified PVA-PEG and amino- $\beta$-CD. ${ }^{104}$

Amiel and coworkers used the interaction of a cationic surfactant, a neutral highly $\beta$-CDmodified polymer and DNA to prepare another type of gene-delivery SNPs. ${ }^{108}$ By supramolecular interactions of $n$-dodecyltrimethylammonium chloride with the $\beta$-CDs grafted to the polymer chain, the hydrophobic tails of the amphiphiles were encapsulated within the cavity of the $\beta-C D$, exposing the cationic heads. These multiple cationic charges led to electrostatic interaction with the DNA going along with SNP formation. To investigate the effect of the amphiphilic head group on the stability of the forming DNASNPs, n-dodecyltrimethylammonium chloride was replaced by different Ad-modified amphiphiles. ${ }^{109}$ Increasing the valency of cationic charges and integration of amide bonds in the hydrophilic tail enhanced the electrostatic interaction and promoted hydrogen bonding with the DNA. This led to an increased stability of the DNA-SNPs and prevented polyplex dissociation under physiological conditions. 


\subsubsection{Multivalent cyclodextrin-stabilized SNPs}

Davis and coworkers have reported the formation of SNPs stabilized by multiple supramolecular host-guest interactions of a single polymer strand (Figure 2.9). ${ }^{110}$ Hereto, a linear, highly water soluble and non-biodegradable polymer containing alternating cyclodextrin and PEG segments was designed. ${ }^{111}$ This polymer was further functionalized with camptothecin (CPT), a drug with anti-cancer activity. Self-assembly of this $\beta-C D$ and CPT-functionalized polymer in aqueous solution resulted in SNP formation with particle sizes of 30-40 nm. Due to the stability of the SNPs in water and DMSO and the selective SNP disassembly by addition of a competing guest molecule, the SNP selfassembly was attributed to host-guest complexation. Drug release experiments have shown that the CPT can be cleaved off from the polymer chain while deforming the SNPs. The supramolecular polymer was designed such that the CD-CPT-SNPs have long in vivo circulation times, whereas the $\mathrm{CD}$ polymer is renally cleared after the elimination of CPT. ${ }^{112}$ These CPT-SNPs, called CRLX101 (previously IT-101), are currently in clinical trials for the treatment of patients with malignancies. ${ }^{113}$ The obtained CPT release kinetics, the overall pharmacokinetics and pharmacodynamic parameters have been correlated to the positive results obtained in animal studies. ${ }^{114}$ Owing to the flexibility of the polymer, the antiproliferative peptide tubulysin could be attached to the SNPs, which enhanced the maximum tolerable medication dose. ${ }^{115}$

a)

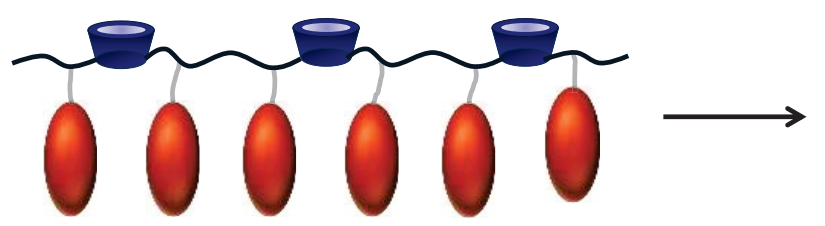

b)
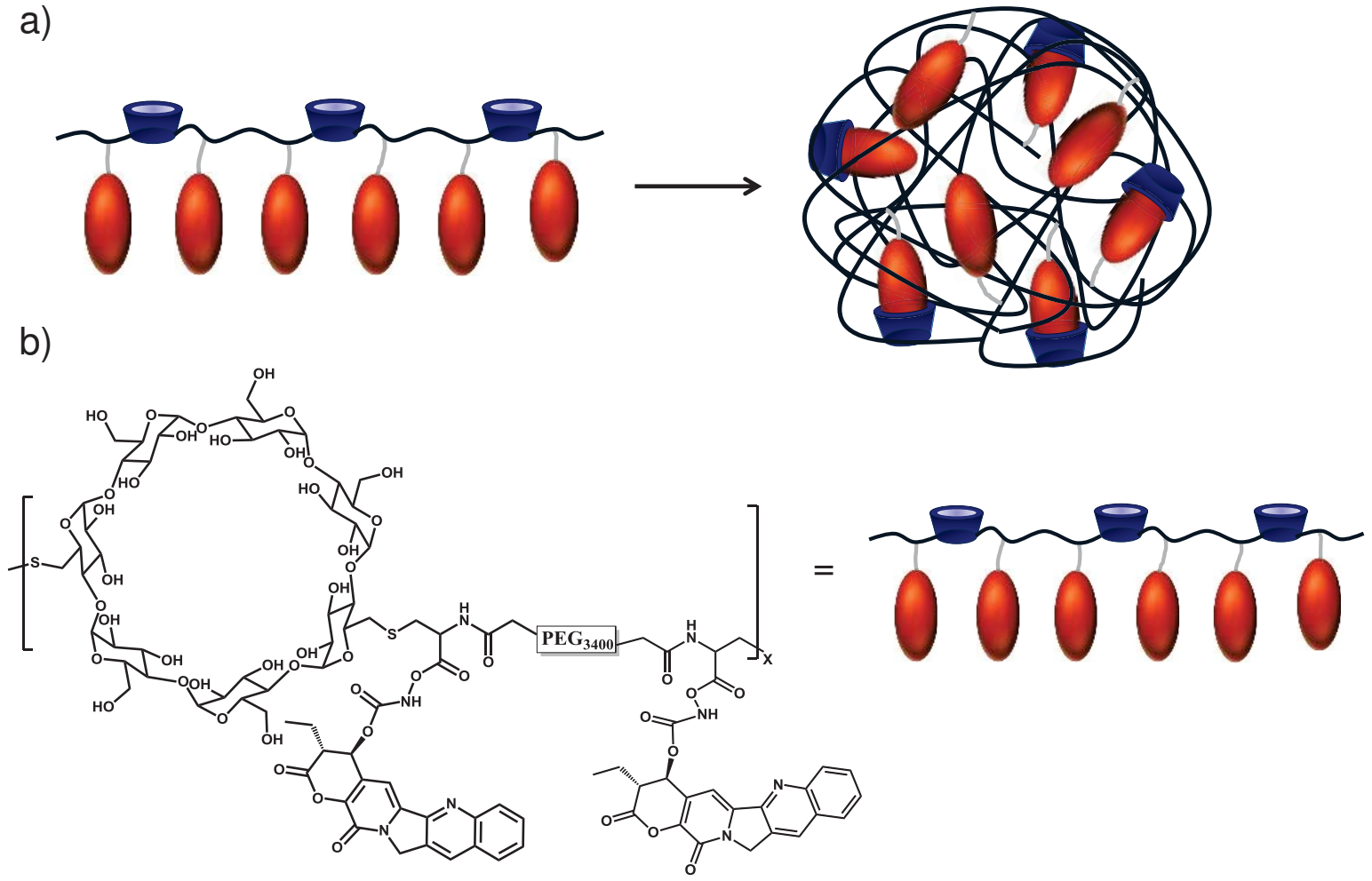

Figure 2.9: a) Formation of a camptothecin (CPT) delivery vector based on intramolecular multivalent interaction of a single CPT- and CD-functionalized polymer chain. b) Structure of CDCPT-PEG. ${ }^{114}$ 
Lu et al. have reported an analogous pathway to prepare CPT and gene co-delivering SNPs. ${ }^{116}$ Low molecular weight PEI was functionalized with $\beta-C D$ and CPT, and intermolecular host-guest interactions led to the formation of SNPs with sizes of approx. $180 \mathrm{~nm}$. The cationic nature of the PEI enabled condensation of p53, a tumor suppressor gene. The size of these co-delivering SNPs ranged from 220-400 nm depending on the content of CPT. Based on the hydrophobic character of CPT, the maximum loading of the polymer with CPT was determined to be $5 \%$. Higher grafting densities resulted in poorly water soluble aggregates. In vivo studies in mice have shown that tumor growth could be suppressed by co-delivery of CPT and p53.

Supramolecular core-shell SNPs stable in aqueous solution are generally prepared by polymers with different hydrophobicities. ${ }^{117}$ To further stabilize such SNPs, Wang et al. have reported core-shell SNPs based on hydrophobic interactions and multiple inclusion complexes between $\beta-C D$ and $A d$ moieties of $\beta-C D$-modified linear poly(glycidyl methacrylate) (PGMA) and Ad-containing poly(tert-butyl acrylate) (PtBA) ${ }^{118}$ As Ad-PtBA is relatively hydrophobic whereas CD-PGMA is hydrophilic, hydrophobic interactions led to the formation of core-shell SNPs, with Ad-PtBA assembled in the core and CD-PGMA in the shell. Residual free $\beta-C D$ moieties in the shell enabled further supramolecular modification of the SNPs by monovalent Ad-containing molecules.

$\mathrm{Ma}$ and coworkers have reported the formation of supramolecular core-shell SNPs by multivalent host-guest interactions. They used the more hydrophobic guest polymer poly( $\beta$-benzyl L-aspartate) (PBLA) assembled in the core of the SNPs while undergoing multiple host-guest interactions with the hydrophilic host polymer $\beta$-CD-polyaspartamidePEG diblock copolymer (Figure 2.10). ${ }^{119,120}$ In addition to the $\beta$-CD moieties also the PEG modification of the $\beta$-CD-diblock copolymer promotes the formation of the SNP shell. Size tunability of these SNPs was observed by varying the ratio of guest to host polymer, the composition of the PEG-CD polymer, and by increasing the molecular weight of the hydrophobic polymer backbone. ${ }^{119,121}$ Temperature-responsive SNPs were obtained by replacement of the poly( $\beta$-benzyl L-aspartate) with poly( $N$-isopropylacrylamide) (PNIPAM). ${ }^{122}$ The same $\beta$-CD-containing diblock copolymer was used as well to encapsulate and release hydrophobic drugs such as pyrene, ibuprofen, indomethacin or dexamethasone upon formation of smaller polymeric aggregates. ${ }^{119,123}$ In contrast, to prepare DNA delivery SNPS, CD-PEI instead of the CD polyaspartamide-PEG diblock copolymer was used in the presence of PBLA to enable electrostatic interactions. The hydrophobic PBLA assembled in the core of the SNPs and was found capable of the inclusion of lipophilic drugs. The hydrophilic CD-PEI assembled at the shell of the SNPS and condensed the DNA. 
a)
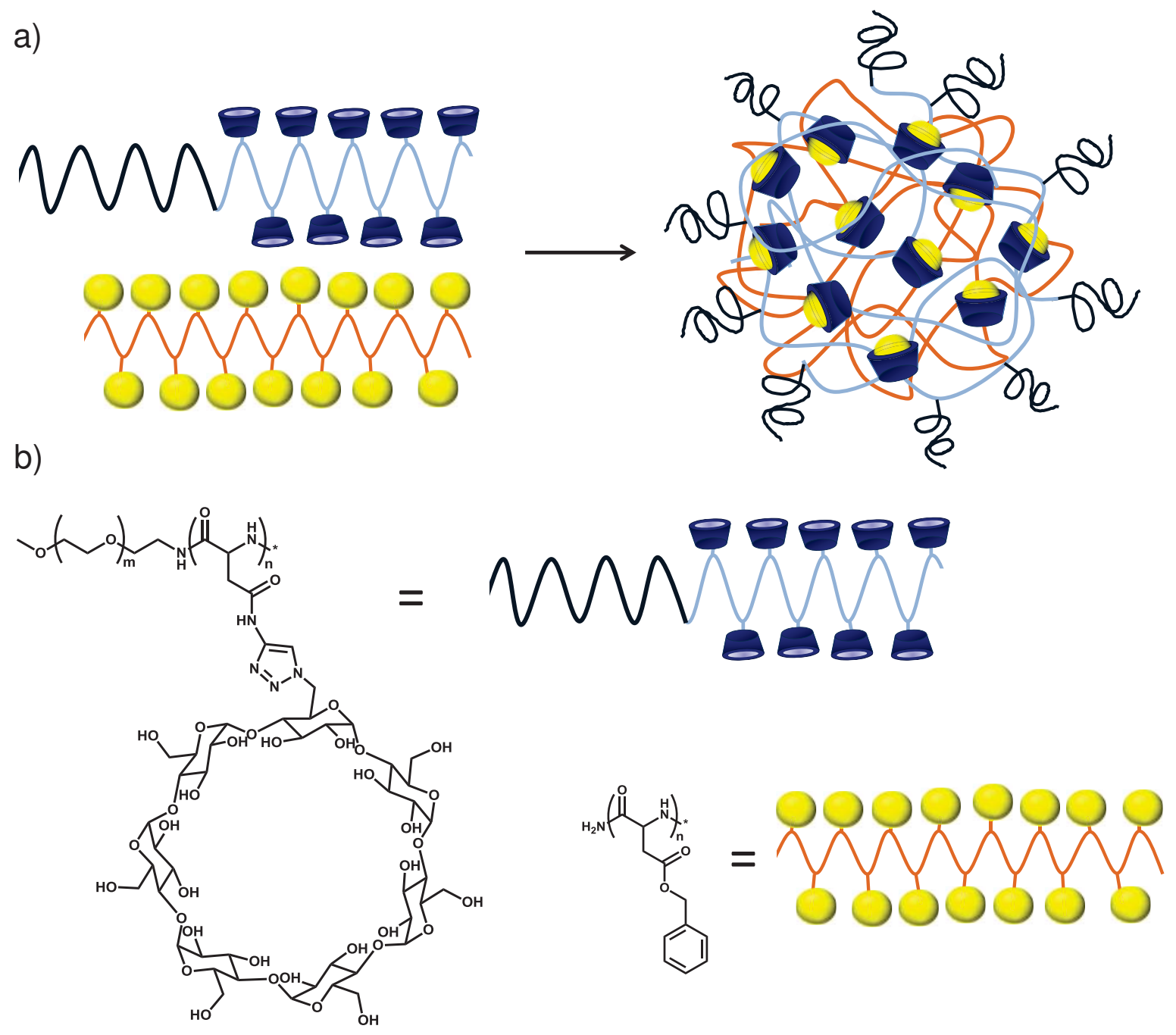

)

Figure 2.10: a) Formation of SNPs by multivalent interactions of B-CD and benzyl groups of a hostand a guest-functionalized polymer. The hydrophilic diblock copolymer contains a PEG block which assembles at the shell of the SNPs and a polyaspartamide block carrying the B-CD units which interact with poly( $\beta$-benzyl L-aspartate) stabilizing the core. b) Structure of poly( $\beta$-benzyl Laspartate) and $\beta$-CD-polyaspartamide-PEG diblock copolymer.

More fundamental studies to understand the formation of multivalently connected SNP assemblies were reported by Couvreur and coworkers. ${ }^{124}$ Here, alkyl chains were grafted onto dextran, and poly- $\beta-C D$ was prepared by crosslinking $\beta-C D$ with epichlorohydrin. Mixing these two polymers led to formation of so-called spherical nanogels. As the size of the formed assemblies was initially smaller than $200 \mathrm{~nm}$, these structures could also be called SNPs. The concentration of the formed SNPs appeared to be crucial for their stability, because the probability of interparticle interaction increases with the overall concentration of building blocks. Furthermore the SNPs were not stable above $60^{\circ} \mathrm{C}$; at this temperature only few alkyl chains were complexed in the $\beta-C D$ cavity, and SNP disassembly occurred. In this study, small drugs were entrapped in the available $\beta$-CDs. This concept was further extended to a magnetic resonance imaging (MRI) contrast agent by integrating $\mathrm{Gd}^{3+}$ in the SNPs. ${ }^{125}$ To this purpose, an Ad-bearing Gd-chelate 
complex was synthesized and integrated into the SNPs by host-guest complexation with the free $\beta$-CDs.

The effects of the molecular structure and the valency of the host-guest interactions on the assembly and stability were studied by using differently modified dextran polymers. To study the effect of guest functionality and charges on the formation of multivalently interconnected SNPS, Amiel and coworkers synthesized $\beta-C D$-functionalized dextran and dextran modified with dodecyl, Ad and cyclohexanecarboxylic acid. ${ }^{126}$ Isothermal titration calorimetry (ITC) experiments showed that, in addition to host-guest interactions of $\beta$ CD with the hydrophobic dodecyl and Ad groups, electrostatic interactions were responsible for SNP stabilization. Exclusively electrostatically stabilized SNPs were highly $\mathrm{pH}$ responsive, and the polymer-polymer association and dissociation mechanism was correlated to the charge densities of the dextran chains. A minimum number of multivalent interactions is apparently required to form stable SNPs, and the size of the SNPs decreases with the valency of the interaction. ${ }^{127}$ By increasing the substitution degree of the host or guest polymer, a smaller number of polymeric chains was involved in the SNP formation, which caused a decrease in SNP size. Simultaneously, the enhanced amount of intermolecular connections led to an increase in SNP stability, which showed that the SNPs were stabilized by multivalent host-guest interactions.

Tseng and coworkers have reported a highly adaptable multi-component approach for the preparation of SNPs by the molecular recognition of Ad and $\beta-C D$ moieties. Based on a three-component system consisting of a multivalent $\beta-C D$ polymer (CD-PEI), Adfunctionalized dendrimer ( $\left.\mathrm{Ad}_{8}-\mathrm{PAMAM}\right)$, and Ad-PEG, size-tunable SNPs were formed (Figure 2.11). ${ }^{128}$ The SNPs assemble by multivalent interactions of CD-PEI with $\mathrm{Ad}_{8}$ PAMAM in the core and an excess of monovalent interactions of CD-PEI with Ad-PEG in the shell. The monovalent hydrophilic polymer enables dispersion of the SNPs in aqueous solution, prevents uncontrolled aggregation, and enables functionalization of the SNP shell. Furthermore, the interplay between multivalent and monovalent $A d-\beta-C D$ interactions enables size tuning of the SNPs by varying the composition: the more monovalent Ad-PEG present in the SNP formulation the smaller the observed SNP size. By increasing the ratio of $\mathrm{Ad}_{8}$-PAMAM to CD-PEI, while keeping the content of Ad-PEG constant, SNP sizes ranging from $30-450 \mathrm{~nm}$ were observed. To improve the size distribution of the SNPs, a digital microfluidic droplet generator was used in which the processing parameters are precisely controlled and multiple samples can be prepared in parallel. ${ }^{129,130}$ The high reproducibility and the narrow size distribution of the particles indicate that SNP formation can be kinetically controlled within the microreactor. 


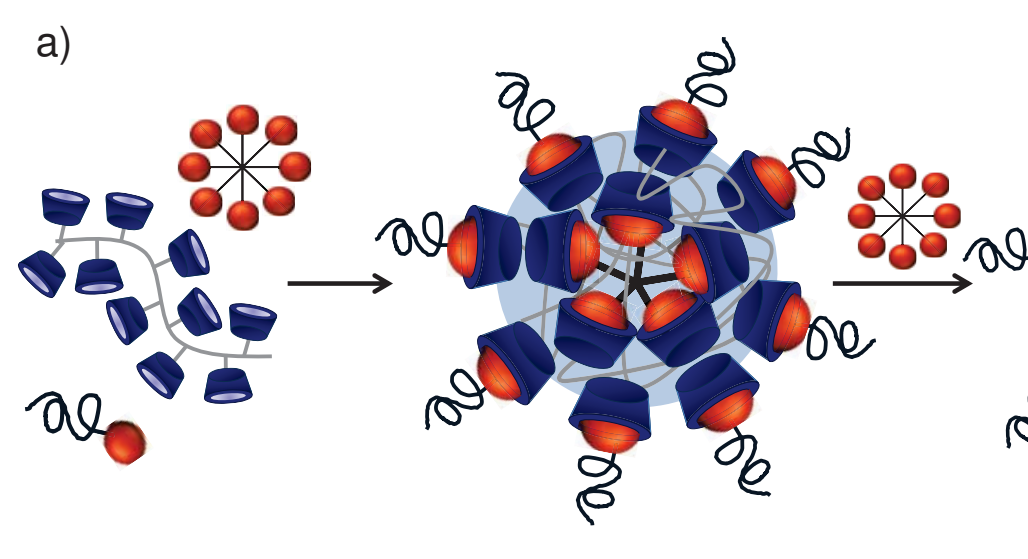

Ad $_{8}$-PAMAM/CD-PEI/Ad-PEG:

b)

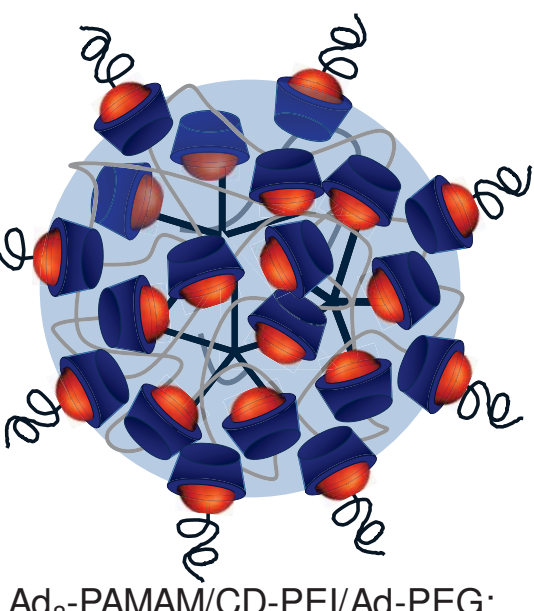

$\mathrm{Ad}_{8}$-PAMAM/CD-PEI/Ad-PEG:

$4: 1: 5$

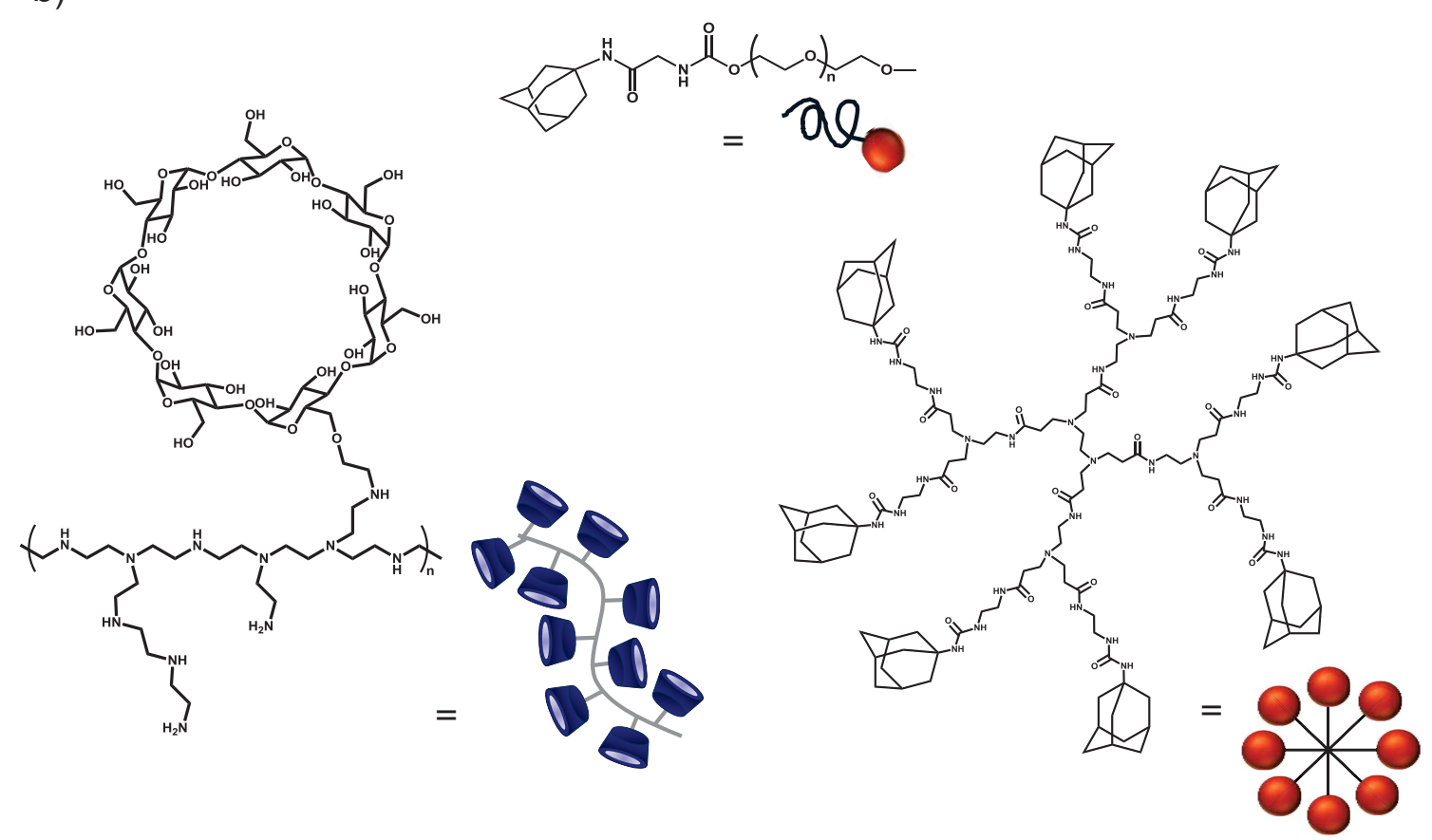

Figure 2.11: a) SNP formation mediated by host-guest interactions of $A d_{8}-P A M A M$ and Ad-PEG with $C D$-PEI. The interplay between multivalent vs. monovalent interactions enables size tuning of the SNPs depending on the ratio of guest building blocks. b) Structures of CD-PEI (average number of 10 CDs per polymer chain), Ad-PEG and $\mathrm{Ad}_{8}-$ PAMAM. ${ }^{128}$

The toolbox versatility of the SNP approach reported by Tseng enabled the implementation of different functional molecules to make these SNPs applicable in biomedical diagnosis and therapy. Chemical modification of CD-PEI with DOTA molecules enabled the introduction of ${ }^{64} \mathrm{Cu}$ and the paramagnetic $\mathrm{Gd}^{3+}$ allowing for application in positron emission tomography ${ }^{128}$ and magnetic resonance imaging, ${ }^{131}$ respectively. A collection of gene delivering SNPs with different sizes and tunable RGD ligand coverage were prepared by DNA condensating using the positively charged CD-PEI and RGDmodified Ad-PEG. ${ }^{132}$ For the delivery of intact transcription factors (TFs), precomplexation of the TF with DNA plasmids containing five matching recognition 
sequences was required to obtain an anionic TF-DNA complex which could be included inside the SNPs. ${ }^{133}$ Electrostatic interactions were used additionally to integrate the negatively charged CPT-modified poly(L-glutamic acid) (CPT-PGA). ${ }^{134}$ The design of CPTPGA enabled controlled drug release by esterase-mediated hydrolysis under physiological conditions in tumor tissue.

The formulation developed for the formation of soft SNPs was extended to the inclusion of inorganic NPs for preparing optically and magnetothermally responsive SNPs. ${ }^{135,136}$ For photothermally responsive SNPs, Ad-grafted Au-NPs with an average size of $2 \mathrm{~nm}$ were prepared. These NPs were included in the SNPs by addition of CD-PEI and Ad-PEG. Similar to the soft SNPs, the size of the Au-SNP clusters is regulated by an equilibrium of monovalent propagation of Ad-PEG and aggregation of the multivalent Ad-Au-NPs. Using Au-SNPs with sizes of $120 \mathrm{~nm}$, generation of microbubbles was observed at a relatively low laser energy. In contrast, no microbubble formation was observed for the individual Ad-Au-NPs at this laser power. The significant enhancement of the photothermal effect in Au-SNPs was attributed to the collective heating effect in the Au-SNPs. To make use of an external alternating magnetic field to selectively disassembly drug-containing SNPs, superparamagnetic $\mathrm{Zn}_{0.4} \mathrm{Fe}_{2.6} \mathrm{O}_{4}$ NPs were modified with Ad and mixed with $\mathrm{Ad}_{8}$-PAMAM, Ad-PEG, CD-PEI and doxorubicine (DOX). ${ }^{136}$ In vivo mouse experiments showed on demand drug delivery and accelerated tumor suppression upon applying an alternating magnetic field.

Our group has recently reported an SNP study in which the function of the monovalently host-guest interacting Ad-PEG was evaluated in more detail. ${ }^{137}$ SNPs were stabilized by multivalent host-guest interaction of $p$-tert-butylphenyl (TBP) and $\beta-C D$ using $\beta-C D$ and TBP-modified poly(isobutyl-alt-maleic acid) (PiBMA) (Figure 2.12). In water, the size and stability of the SNPs was independent of the addition of Ad-PEG, which was attributed to electrostatic repulsion of the polymer backbones preventing the addition of more polymer chains to the outer shell of the SNPs. In contrast, while increasing the ionic strength of the SNP medium, the SNPs destabilized and aggregated. This aggregation process was prevented if Ad-PEG was added to the SNP solution, while keeping the hostguest ratio constant. Thus, it can be concluded that the size and stability of SNPs are controlled by a balance of attractive and repulsive supramolecular host-guest interactions, and that the size tunability reported by Tseng and coworkers is only effective if repulsive electrostatic interactions are not dominating. 
a)

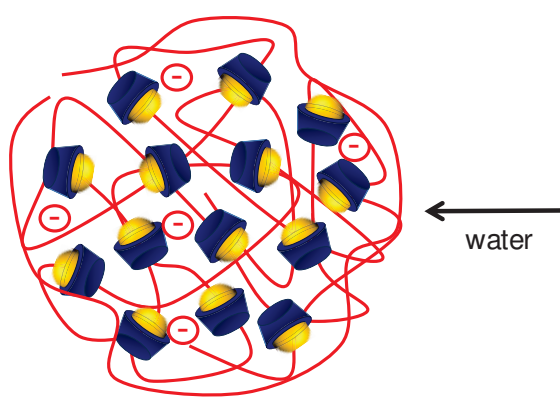

b)

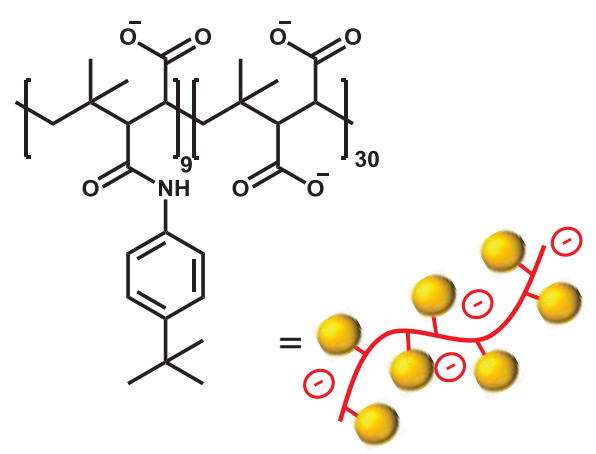
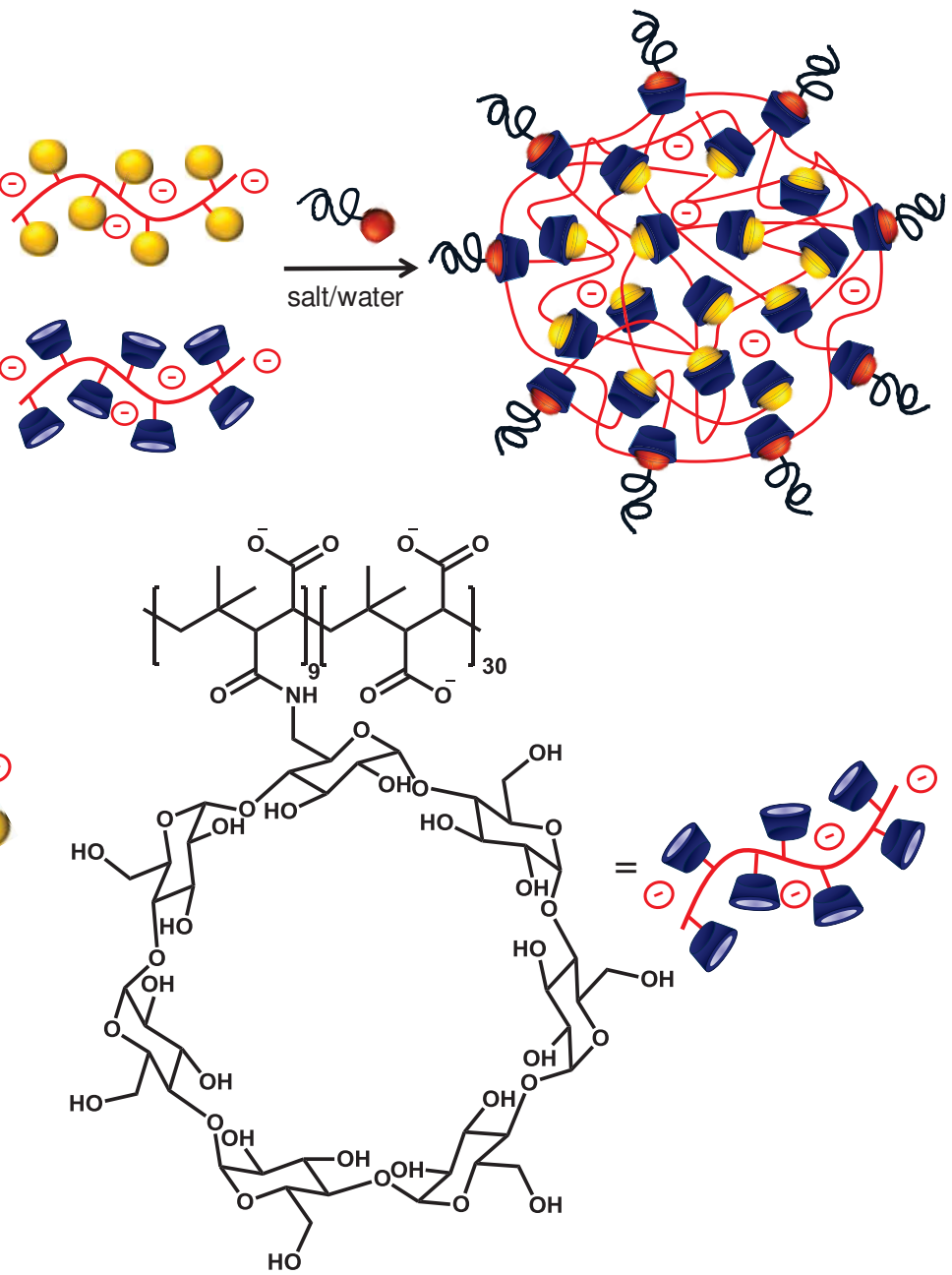

Figure 2.12: a) Schematic representation of SNP formation from poly(isobutyl-alt-maleic acid) (PiBMA) grafted with $\beta$-CD as the host polymer, PiBMA functionalized with p-tert-butylphenyl (TBP) as the guest polymer and Ad-PEG. b) Structures of TBP-PiBMA and CD-PiBMA. ${ }^{137}$

Soft hollow microcapsules were formed by host-guest interaction of two electrostatically repulsive polymers. ${ }^{138}$ Using an $\mathrm{LbL}$ approach, $\beta-C D$ and ferrocene-modified poly(allylamine hydrochloride) (PAH) polymers were coated around $\mathrm{CaCO}_{3}$ particles. To obtain hollow microcapsules, the $\mathrm{CaCO}_{3}$ core was removed by ethylene diamine tetraacetate (EDTA). The thickness of the microcapsule shell was tuned between 5-50 $\mathrm{nm}$ depending on the number of assembly cycles. Based on the underlying electrostatic repulsion and host-guest interactions the formed hollow capsules showed capsule swelling induced by a decrease of $\mathrm{pH}$ and by the addition of a host competitor. To implement switching by light, $\mathrm{a}-\mathrm{CD}$ and azobenzene (Azo) were used as host-guest recognition motif. ${ }^{139}$ By irradiating the capsules with UV light, isomerization of trans-Azo into its cis isomer was induced and the host-guest complex was destroyed because of the resulting steric repulsion between cis-Azo and the CD cavity. 


\subsubsection{Cyclodextrin supramolecular nanoparticles stabilized by hydrophobic interactions}

CD-containing SNPs, stabilized by hydrophobic interactions, are mostly based on covalent amphiphiles or non-covalent CD-based amphiphiles called pseudo-amphiphiles. As schematically shown in Figure 2.13, the CD cavity acts as the hydrophilic fragment and either covalently attached aliphatic chains or the guest molecule act as the hydrophobic fragment in CD amphiphiles. In water, the covalent amphiphile and the pseudoamphiphile can form different supramolecular assemblies based on hydrophobic interactions.

a)
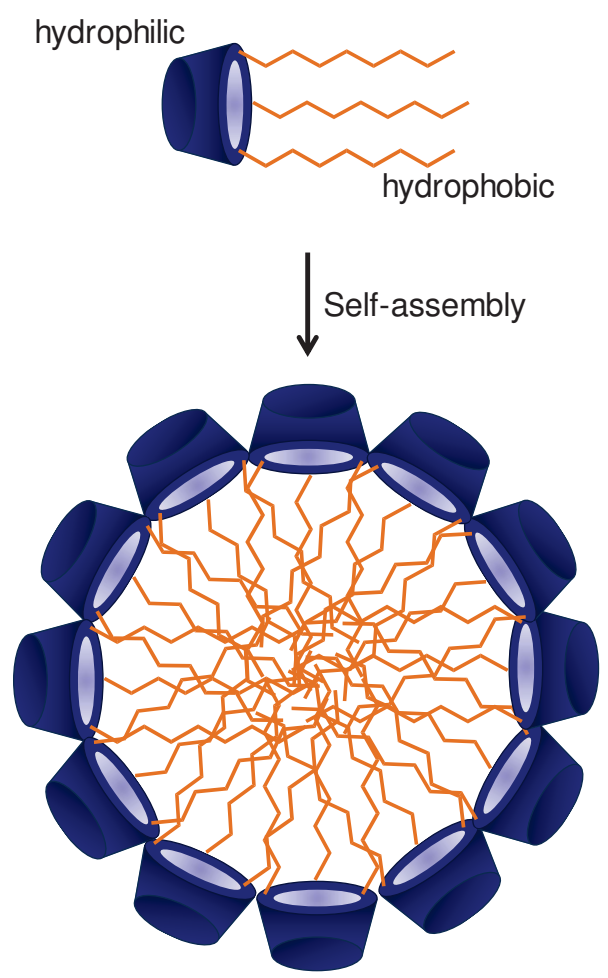

b)
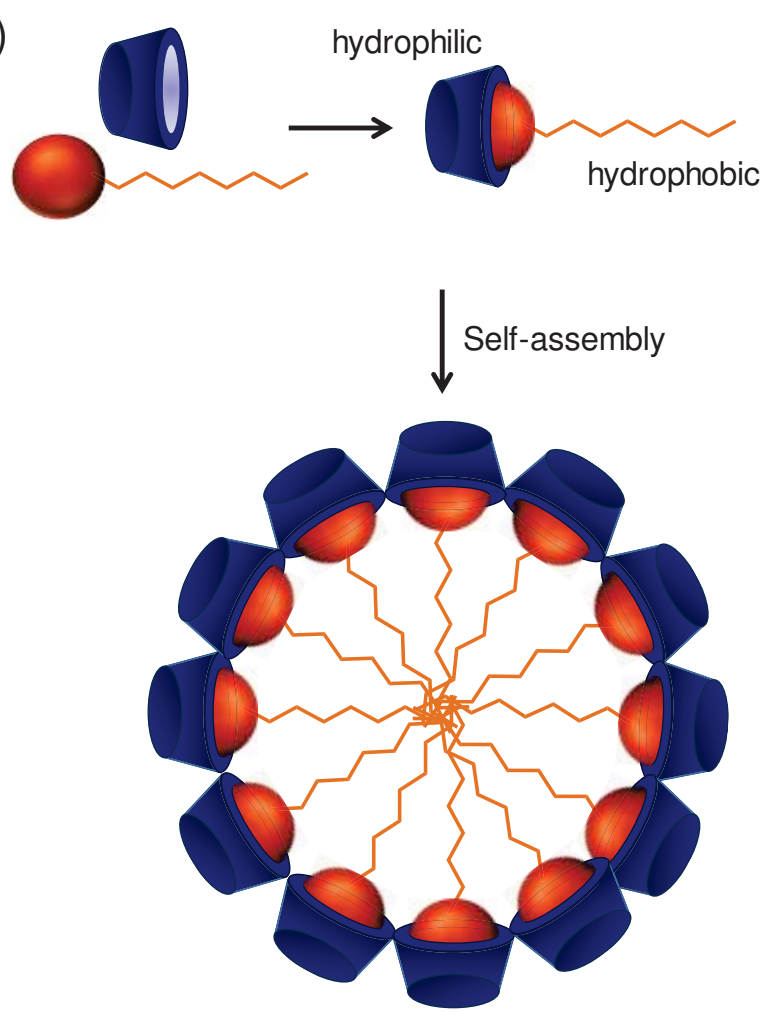

Figure 2.13: Schematic representation of CD-based micelle formation by a) covalently modified $C D$ amphiphiles and b) amphiphiles formed by host-guest inclusion complexation.

Chemical modification of the CD host molecules is not new and the synthesis of supramolecular amphiphiles by grafting one or more hydrophobic groups to the cyclodextrin ring was already reported in $1991 .{ }^{140}$ Accordingly, the aggregation behavior of these structures was studied heavily over the past 20 years. Depending on the conformation of the CD-containing amphiphile, supramolecular micelles, ${ }^{141-143}$ supramolecular vesicles ${ }^{144}$ or rod-like structure are formed in aqueous medium. These structures were used, for example, as drug delivering vesicles ${ }^{145}$ or for the photoresponsive release of entrapped DNA. ${ }^{146}$ In contrast, a pseudorotaxane formed by inclusion complexation can act as an amphiphile, if a considerable part of the 
hydrophobic tail is exposed outside the cavity. Jiang and Chen summarized the efforts made in the field of CD-containing micelles, including systems requiring host-guest precomplexation prior to self-assembly in water. ${ }^{147,148}$

By focusing on responsive pseudorotaxane-based amphiphilic systems, polymer properties and the underlying host-guest mechanism, micelle and vesicle association and dissociation can be controlled selectively. Zeng et al. reported the formation of supramolecular micelles by the formation of host-guest complexes between $\beta$-CD-poly(4vinylpyridine) (CD-P4VP30) and Ad-containing PNIPAM (Ad-PNIPAM). ${ }^{149}$ The association of the formed amphiphiles leading to the supramolecular structure, was found to be strongly dependent on temperature and $\mathrm{pH}$. At room temperature and $\mathrm{pH} 4.8$, the amphiphilic complexes self-assembled into a vesicle structure under the formation of a supramolecular double layer. A decrease to $\mathrm{pH} 2$ or an increase in temperature led to increase of the solubility of P4VP30 in water and thus transformation of the vesicles into micelles. According to this procedure, Liu et al. synthesized $\beta$-CD-PNIPAM and Adfunctionalized poly(2-(diethylamino)ethyl methacrylate) (Ad-PDEA), which formed micelles or vesicles depending on temperature and $\mathrm{pH}$ of the aqueous solution. ${ }^{150}$ Variation of the host-guest motif was used to selectively disassemble micelles formed by host-guest interaction of $\beta$-CD-poly(styrene) (CD-PS) and ferrocene-poly(ethylene glycol) (FC-PEG). ${ }^{151}$ The inclusion complex of FC and $\beta$-CD is stable but can be easily dissociated by oxidation of Fc. Using this principle, reversible assembly and disassembly of supramolecular vesicles was shown by applying an alternating voltage. Furthermore, drug release profiles were tuned by the value of the oxidizing potential.

\subsubsection{Cyclodextrin supramolecular nanoparticles stabilized by electrostatic interactions}

Single CD molecules bearing cationic charges can be used for the so-called compaction of DNA as cationic polymers and lipids. To this purpose, CDs were modified with positively charged functional groups which form SNPs in the presence of negatively charged DNA. ${ }^{152}$ Reineke and coworkers reported the functionalization of $\beta$-CD with oligo(ethylene imine) moieties to form $\beta$-CD cationic molecules in which the number of charges can be tuned by varying the length of the ethylene imine chain. ${ }^{153}$ Complexation of DNA with the different $\beta-C D$ cations led to SNPs with an average diameter of 80-130 nm. Cell transfection studies have shown that the best transfection efficiency is obtained if the $\beta$ $C D$ is modified with oligo(ethylene imine)s bearing four or five protonated amines per chain. This result shows that multiple charges are required to form stable and effective DNA delivery vectors. Next to amino-functionalized CDs, also guanine-functionalized a-, $\beta$ - and $y$-CDs were used to prepare electrostatically stabilized SNPs. ${ }^{154,155}$ Depending on 
the structure of the guanine unit, enhanced SNP cell penetration was reported along with DNA transfection.

DNA and DOX co-delivering SNPs were prepared using PEI modified with multiple $\beta-C D$ host molecules, a monovalent DOX-grafted Ad guest molecule (Ad-DOX) and DNA. ${ }^{156,157}$ The SNPs were prepared in two steps by host-guest interactions of Ad-DOX with CD-PEI, followed by condensation of the DNA, whereby the electrostatic interactions are the main driving force for stabilization of the SNPs. Although drug polymer conjugates with increased drug loading amounts could be prepared, DNA condensation and SNP formation required sufficient positive charges which are diminished by drug loading. The optimized SNP formulation was subsequently studied in order to monitor the drug and DNA co-delivery. Selective dissociation of the electrostatically stabilized SNPs could be monitored at reduced $\mathrm{pH}$ values. DNA release as well as host-guest bound DOX release was shown to be strongly dependent on the $\mathrm{pH}$. In vivo experiments in mice indicated that these DOX-DNA-SNPs have a synergistic effect on tumor treatment, showing prolonged chances of survival.

\subsubsection{Calix[n]arene and pillar[n]arene supramolecular nanoparticles}

Calix[n]arenes are macrocyclic host molecules, which consist of a varying number of phenolic units ( $n=4,5,6$ or 8 repeating units). Their structure is composed of a hydrophobic upper and a hydrophilic lower rim which are surrounding the hydrophobic hollow cavity (Figure 2.14). Stabilized by hydrophobic effects, ion-dipole interactions and/or hydrogen bonding, ions or small molecules, such as sugars, amino acids, peptides, hormones, drugs ${ }^{158}$ and proteins, ${ }^{159}$ can form inclusion complexes with these supramolecular host molecules. A relatively new class of supramolecular structures are pillar[n]arenes which are structurally comparable to calix[n]arenes. The synthesis of the first pillar[n]arene, 1,4-dimethoxypillar[5]arene, was reported in 2008 by Ogoshi et al. ${ }^{160}$ The hydroxyl functionality of the rims enables simple modification of this host and offers therefore a broad scope of host-guest complexes formed by these hosts. ${ }^{161}$

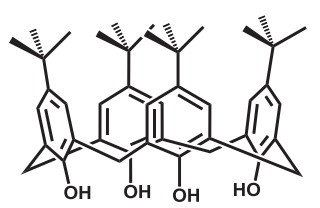

calix[4]arene

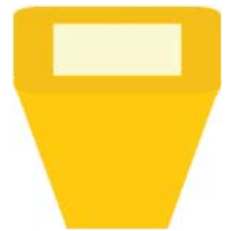

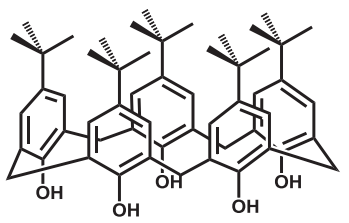

calix[5]arene

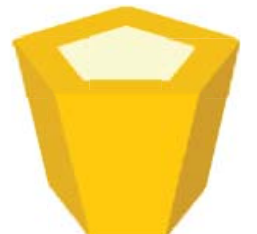

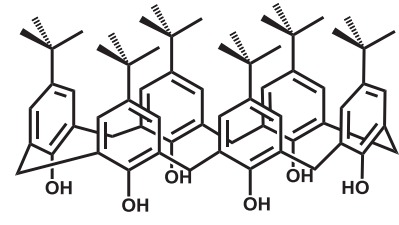

calix[6]arene

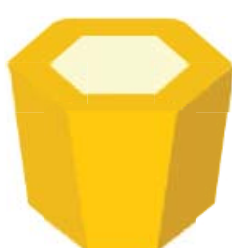

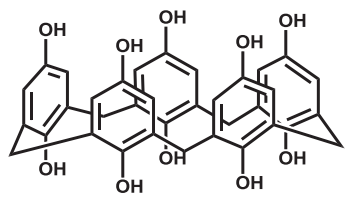

pillar[5]arene

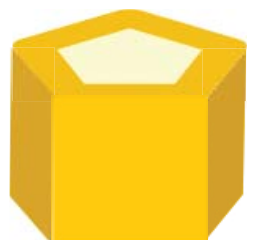

Figure 2.14:Molecular structures and cartoon illustrations of calix[n]arenes $(n=4,5,6)$ and pillar[5]arene 


\subsubsection{Multivalent calix[n]arene/pillar[n]arene-stabilized supramolecular nanoparticles}

Recently, $\mathrm{Xu}$ and coworkers have reported the formation of SNPs for photodynamic therapy by multiple calix[n]arene and methyl viologen (MV) host-guest interactions. ${ }^{162}$ Tetrameric calix[4]arene porphyrin and three different MV guest molecules were synthesized und used to study the selective self-assembly. As schematically shown in Figure 2.15, the tetravalent host molecule forms SNPs in the presence of the divalent MV guest molecule by oligomerization of the building blocks initiated by supramolecular hostguest interaction. To induce SNP formation, the host and guest components were mixed in a 1:4 stoichiometry which led to an overall excess of guest molecules in the system.

a)
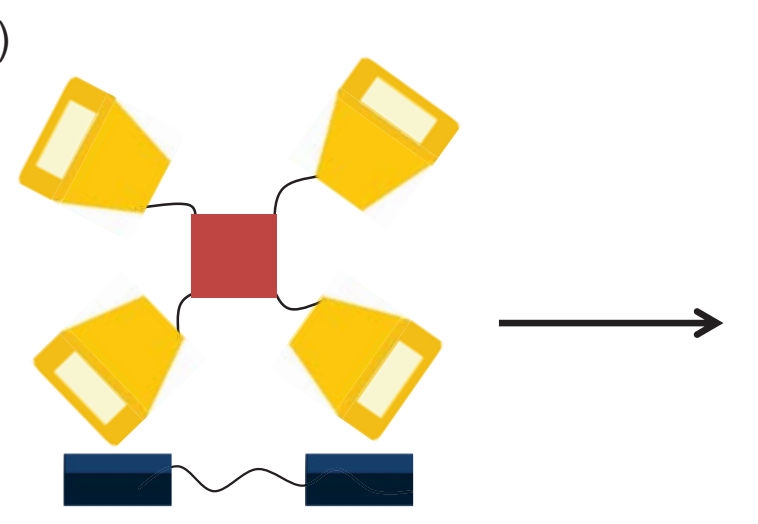

b)
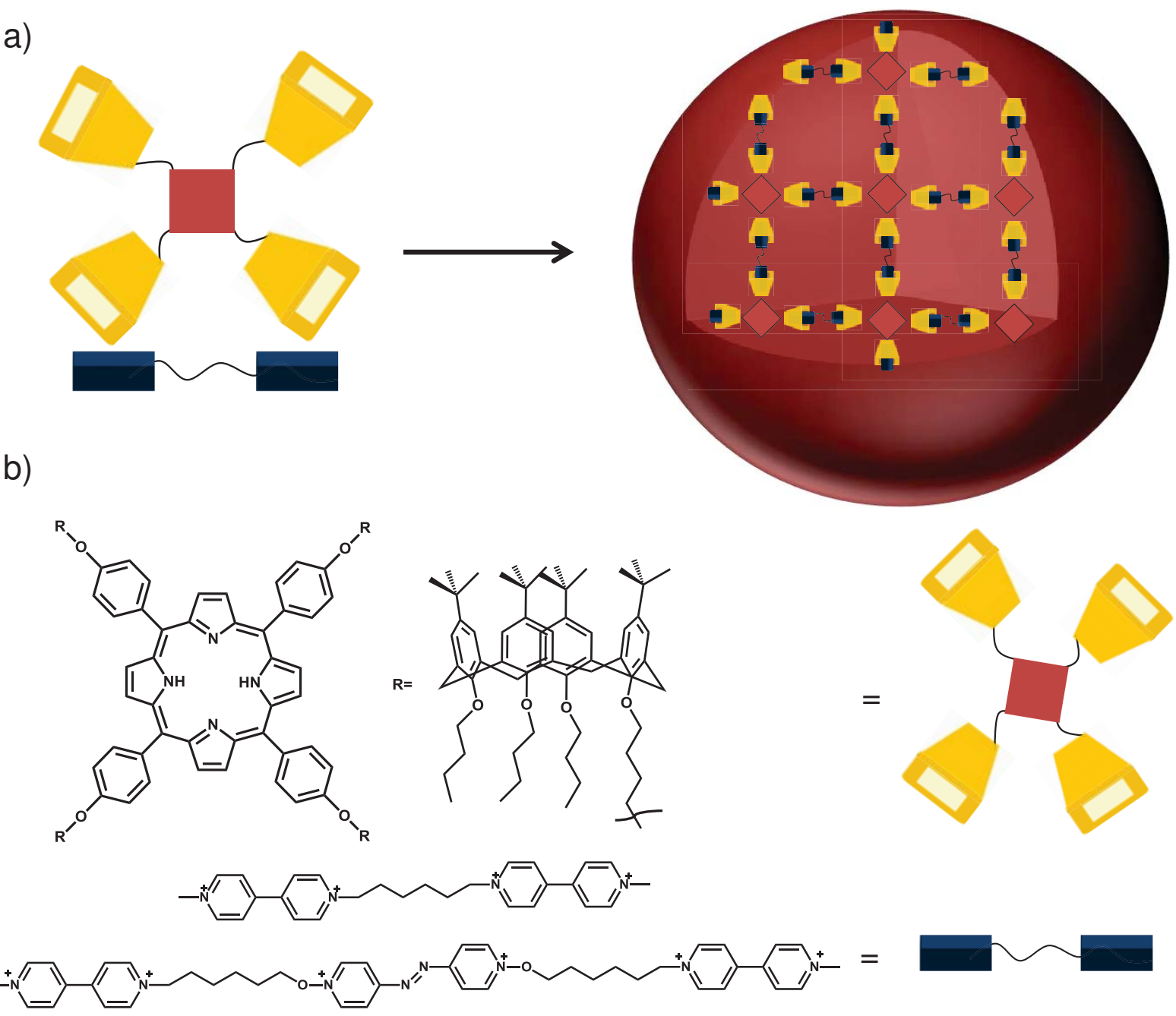

Figure 2.15: a) Schematic representation of the formation of spherical SNPs by host-guest interaction of a tetravalent calix[4]arene-functionalized porphyrin derivative with different bisviologen guest molecules. b) Structure of the tetra-calix[4]arene-substituted porphyrin and the divalent viologen derivatives with different lengths. ${ }^{162}$ 
Irrespective of the stoichiometry and length of the MV guest molecule, size tunability of the SNPs was observed by using different concentrations of both host and guest components. In contrast to previously reported studies carried out with a pillar[5]arene trimer and bis-viologen, ${ }^{163}$ the morphology of the self-assembled structure did not vary by changing the concentration. The covalent integration of porphyrin into the host bearing molecule enabled the application of these SNPs in photodynamic therapy. Generally, porphyrins strongly absorb light which is converted into heat in the illuminated areas. In in vitro cell internalization studies using HeLa cells, the SNPs showed a stronger photo-response than the free porphyrin derivative. Their increased cytotoxicity makes these SNPs a promising candidate for photodynamic therapy.

\subsubsection{Calix[n]arene and pillar[n]arene supramolecular nanoparticles stabilized by hydrophobic interactions}

The formation of amphiphilic structures by covalently modified calix[n]arenes is well known. ${ }^{164-166}$ For example, basic amphiphilic calix[n]arenes which self-aggregate in water into micelles were synthesized by the modification of the lower calix[n]arene rim with four undecyl chains and the upper rim with di- or oligosaccharides. ${ }^{167}$ Also pillar[5]arene was covalently modified with amino groups at the top and alkyl groups at the bottom to form amphiphilic molecules that self-assemble in water into vesicles, sheets and microtubes. ${ }^{168}$ The use of host-guest chemistry enabled thus an enhanced complexity and various applications of amphiphilic calix[n]arene-based SNPs, micelles and vesicles.

Amphiphilic supramolecular complexes were formed by host-guest interaction of the porphyrin-type photosensitizer Chlorin $e_{6}$ with PEG-modified calix[4]arene. ${ }^{169}$ The formation of these host-guest-based amphiphilic structures is illustrated in Figure 2.16. After initial host-guest complexation, the amphiphilic complexes self-assemble into micelles with a size of $140 \mathrm{~nm}$ by hydrophobic interaction. The PEG chains of the modified calix[4]arene ensured water solubility and low toxicity of the micelles which was evaluated by a cell viability assay on HeLa cells. Furthermore, in vitro tests showed the successful uptake of supramolecular micelles by HeLa cells. Cell proliferation could be inhibited by photoirradiation, which makes these supramolecular micelles applicable as drug delivery vehicles for photodynamic therapy. 


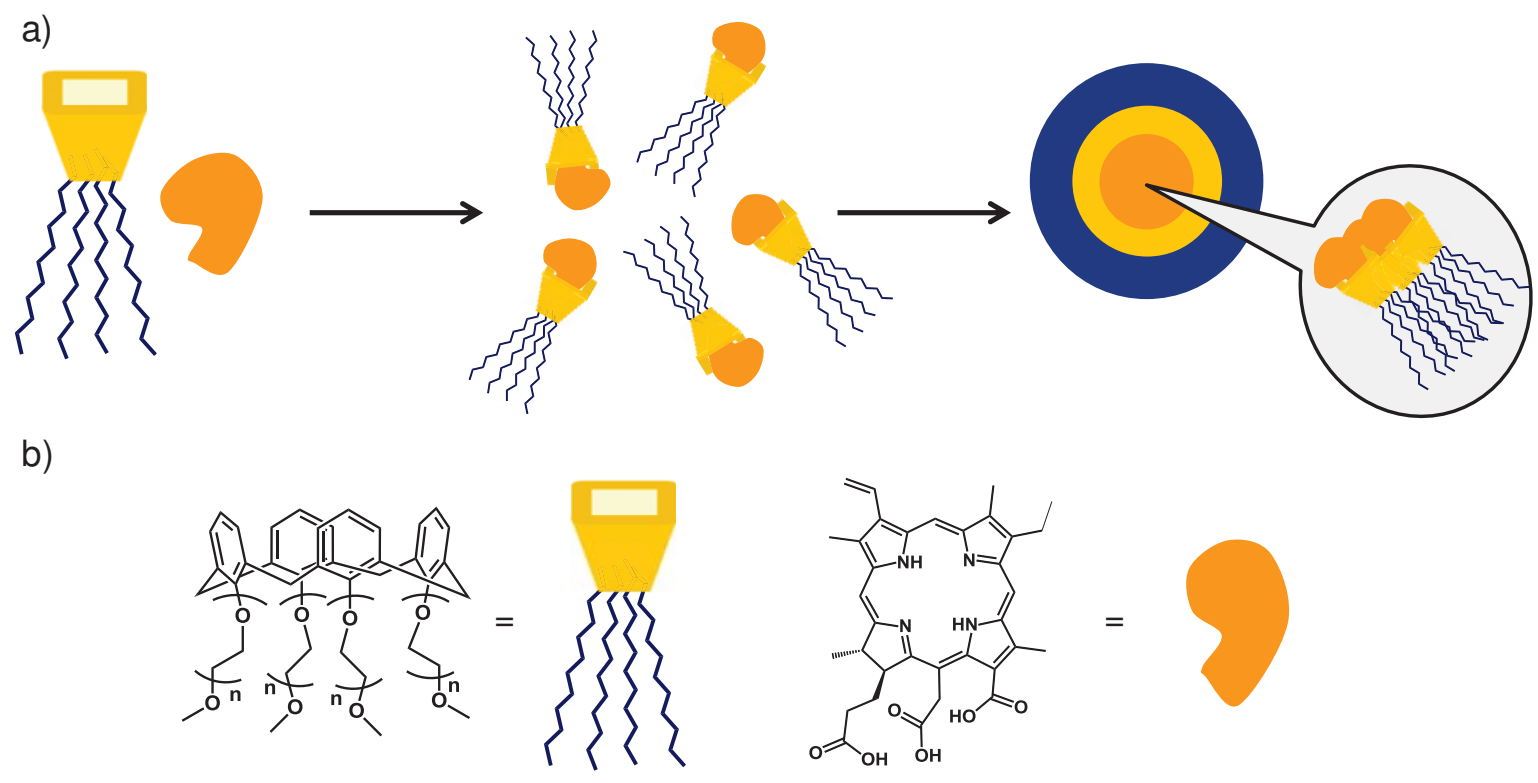

Figure 2.16: a) Schematic representation of the formation of supramolecular micelles by host-guest complexation of PEG-modified calix[4]arene and the porphyrin photosensitizer Chlorin $e_{6}$ followed by micelle formation driven by hydrophobic interactions between the formed supramolecular amphiphiles. b) Chemical structures of PEG-modified calix[4]arene and Chlorin $e_{6}$.

As described for CD-containing amphiphiles, host-guest chemistry enables the modification of calixarene-based supramolecular structures with stimuli-responsive properties. Different systems have been reported in the literature in which the disassembly of amphiphilic micelles and vesicles has been induced by enzymes ${ }^{170,171}$ or a change in temperature. ${ }^{172}$ More interestingly, multi-responsive, drug-encapsulated supramolecular calix[4]arene vesicles were reported by Wang et al. ${ }^{173}$ The formation of these vesicles was induced by the host-guest interaction of $p$-sulfonatocalix[4]arene and decyl-modified viologen. An increase in temperature led to vesicle rupture as the encapsulation of viologen in sulfonatocalix[4]arene and the pi-pi stacking weaken upon heating. In contrast, the reduction of the viologen guest to its radical cation did not lead to disassembly of the vesicles but only to a decrease in vesicle size from 300 to $150 \mathrm{~nm}$. The supramolecular host-guest interaction still persisted, whereas the weakened electrostatic repulsion led to a higher curvature and a concomitant decrease of the vesicle size. Upon a two-step reduction of the viologen unit to the neutral form, the hostguest interaction was completely destroyed and the vesicles fell apart. The same behavior was observed when an excess of calix[4]arene host or a competing a-, $\beta-$, or $\gamma^{-}$ $\mathrm{CD}$ host molecule was added to the system. The CD hosts interacted with the aliphatic chains of the viologen species, which blocked the hydrophobic interaction, essential for vesicle stabilization. This supramolecular approach shows potential for the formation of drug and dye-encapsulating vesicles, for which the release of the encapsulated molecules can be triggered by external stimuli. 
The formation of supramolecular vesicles and fibers based on amphiphilic molecules composed of two different hosts was already reported in $1999 .{ }^{174}$ Recently this concept was further developed to the formation of drug-binding core-shell SNPs based on strong hydrophobic interactions. ${ }^{175}$ Hereto, an amphiphilic heterodimer with a hydrophobic calix[4]arene and a hydrophilic $\beta-C D$ moiety was synthesized. In aqueous solution, the formation of the SNPs is driven by the strong hydrophobic interaction between the multiple carbon chains attached to the calix[4]arene fragment. The SNPs presented the hydrophilic $\beta$-CDs at the SNP shell, which was used for targeting by the interaction with a sugar-modified Ad guest molecule. NP precipitation, caused by a change of solvent, was used to form drug-loaded SNPs of the heterodimer in the presence of the antimitotic agent docetaxel (DXT). Drug release profiles were shown to depend on the length of the hydrophilic alkyl chain grafted onto the calix[4]arene moiety. As DXT was assembled in the SNPs by two different interactions, initial fast release was observed for drugs encapsulated in the shell by host-guest interactions, whereas core-encapsulated molecules were released more slowly.

Zhao and coworkers reported the formation of pillar[5]arene assemblies mediated by hydrophobic interactions of different amphiphilic pillararenes in aqueous solution. ${ }^{176}$ Tadpole- and bola-like pillar[n]arenes were functionalized with hydrophilic ethylene glycol and hydrophobic alkyl groups. Depending on the pillar[n]arene design, the amphiphilic supramolecular hosts assembled in the absence or presence of a supramolecular guest molecule. Interestingly, the structure of the amphiphile also determined the encapsulation efficiency of different dyes and DOX in these vesicles. For example, all supramolecular assemblies could encapsulate rhodamine $B$, whereas only the tadpole-like amphiphilic pillar[n]arene-based assemblies included and delivered the hydrophobic dye fluorescein isothiocyanate to cells in vitro. To prove that these assemblies can be used for drug delivery applications, DOX was encapsulated and delivered to cells in vitro. Apparently, the structure of the amphiphilic pillar[n]arene has a strong impact on the successful formation and performance of supramolecular assemblies for bioimaging and drug delivery.

Recently, the formation of supramolecular amphiphiles stabilized by host-guest interaction of pillar[5]arene with an imidazole-containing rod-coil molecule was reported. ${ }^{177}$ Due to electronic de-coupling of the quinquephenyl aromatic rings in the divalent imidazole-containing guest molecule, the fluorescence intensity increased drastically upon supramolecular recognition. Due to the stimulus-responsive character of the host-guest recognition motif, vesicle disassembly could be induced in three ways: by addition of the supramolecular competitor paraquat, by a decrease in $\mathrm{pH}$, or by addition of silver oxide. ${ }^{178}$ 


\subsubsection{Calix[ $n$ ]arene supramolecular nanoparticles stabilized by electrostatic interactions}

The groups of Casnati and Ungaro have reported the synthesis of a series of guanidinium modified calix[n]arenes for the formation of SNPs in the presence of DNA, driven by hydrophobic and electrostatic interactions. ${ }^{179,180}$ Different calix[n]arene derivatives were synthesized and their DNA condensation and cell transfection efficiencies were tested depending on the calix[n]arene lipophilicity, size, and conformation. AFM studies showed that upper rim guanidinium-functionalized calix[4]arenes, functionalized with lipophilic hexyl or octyl chains at the lower rim, gave DNA condensation and promoted cell infection. ${ }^{180}$ This process is driven by electrostatic interaction of the DNA with the guanidinium units and hydrophobic interaction of the lipophilic tails bound to the calix[4]arene. In contrast, larger guanidinium-calix[n]arene homologues with methoxy functionalization at the lower rim did not result in cell transfection, as these host molecules did not successfully compact the DNA. Only loosely self-assembled structures were formed by electrostatic interaction of the guanidinium groups with DNA filaments. Interestingly, the functionalization of the lower rim of the calix[4]arene with guanidinium instead of the upper rim yielded non-viral vectors with lower toxicity and high transfection efficiency. ${ }^{181}$ More recently, it was shown that DNA condensation, cell transfection and toxicity also depend on the modification of the upper rim of the guanidinium calix[4]arene. ${ }^{182}$ Recently, the same group has shown that a calix[4]arene modified with four arginines at the upper rim and four lipophilic tails at the lower rim enables high DNA condensation efficiency. Moreover, the enhanced transfection efficiency of these SNPs is caused by the host modification with a cell penetrating peptide. ${ }^{183}$

A combination of hydrophobic and electrostatic interactions was used to induce the assembly of calix[4]arene-based, virus-sized DNA NPs. ${ }^{184}$ Calix[4]arene was modified with alkyl chains at the lower rim and cationic groups at the upper rim. Due to hydrophobic interactions these amphiphilic host molecules assembled in water into very small micelles with an average diameter of $6 \mathrm{~nm}$. Upon addition of DNA to these micelles, larger DNA-micelle complexes of approximately $50 \mathrm{~nm}$ were formed by electrostatic interactions of the DNA with multiple cationic groups at the micelle shells. These SNPs showed gene transfection efficiency in cell cultures and low cytotoxicity. As a control, a poor gene delivery composite was obtained for an amphiphilic calix[4]arene modified with short alkyl chains. These structures did not form micelles in water and consequently were not capable of condensing DNA since multivalent electrostatic interactions could not be formed. 


\subsubsection{Cucurbit[n]uril supramolecular nanoparticles}

Relatively recently, the cucurbit[n]uril family (Figure 2.17) has attracted a lot of attention owing to its exceptional molecular recognition properties. The high binding affinities and the tunable association and dissociation properties make them of high interest for responsive SNP systems. Cucurbit[n]urils are pumpkin-shaped macropolycyclic compounds composed of n-glycouril units interconnected with methylene bridges. Their hydrophobic interior is surrounded by two equally sized, carbonyl-laced portals, which allows the inclusion of hydrophobic molecules, and of charged molecules such as various alkyl- and aryl-ammonium ions. In the series of $\mathrm{CB}[5]$ to $\mathrm{CB}[10]$, the internal cavity size increases from $4.4 \AA$ to $10.7-12.6 \AA$ as the cavity entrances increase from $2.4 \AA$ to 9.0 $11.0 \AA{ }^{185,186}$ The host-guest chemistry of the different homologues, as reviewed by the groups of Kim and Isaacs, ${ }^{185,186}$ is strongly dependent on their internal cavity size. Whereas $\mathrm{CB}[6]$ forms stable inclusion complexes with a protonated aminoalkane, $\mathrm{CB}[8]$ and $\mathrm{CB}[10]$ are capable of including two guest molecules simultaneously, either in a homotopic (e.g. an amino acid) or heterotopic fashion. In the latter case, typically an electron-poor and electron-rich guest are co-included as a charge-transfer complex. ${ }^{187}$ Due to its diversity, the cucurbit[n]uril family has emerged as a smart tool kit for the formation of supramolecular architectures in solution. ${ }^{188}$

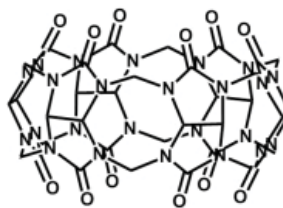

Cucurbit[6]uri

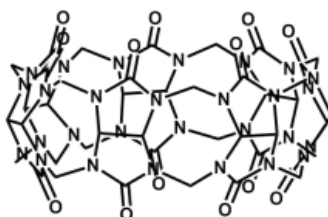

Cucurbit[7]uril

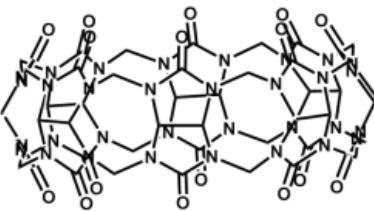

Cucurbit[8]uril

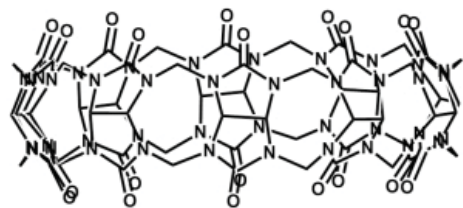

Cucurbit[10]uri

Figure 2.17: Molecular structures of cucurbit[n]urils ( $n=6,7,8,10)$

\subsubsection{Multivalent cucurbit[n]uril-stabilized supramolecular nanoparticles}

SNPs based on $\mathrm{CB}[8]$ crosslinked single polymer chains were reported by the group of Scherman. ${ }^{189}$ A polymer was functionalized with both naphthol (Np) and MV guest moieties, and the formation of multiple, intramolecular, heteroternary charge-transfer complexes induced by $\mathrm{CB}[8]$ led to the collapse of individual polymer molecules into NPs in aqueous solution (Figure 2.18). The size of the resulting SNPs was shown to depend on the molecular weight of the polymer and on the extent of intramolecular host-guest complexation. Similar behavior was shown for a polymer solely functionalized with MV in the presence of nor-seco $\mathrm{CB}[10] .{ }^{190}$ This macrocycle contains two identical cavities and forms 2:1 homo-ternary complexes with two MV guest molecules, which lead to polymer folding going along with SNP assembly. 
a)

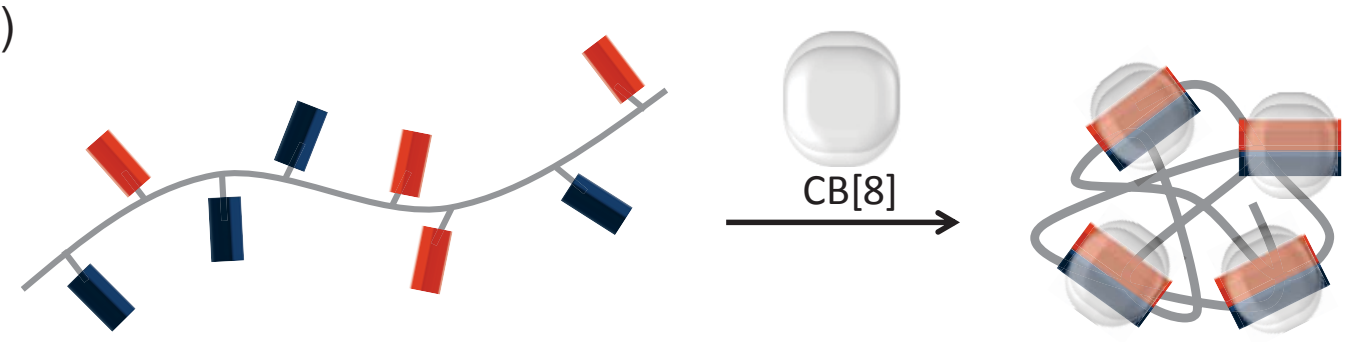

b)

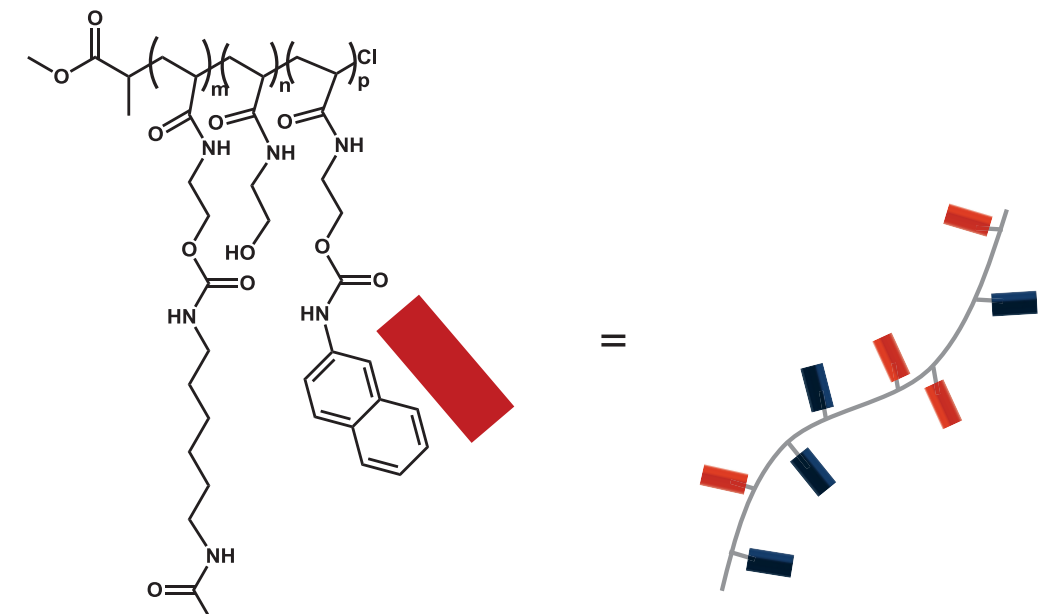

Figure 2.18: a) Single polymer chain SNPs based on multivalent ternary complexation of methyl viologen (MV) and naphthol (Np) moieties with cucurbit[8]uril. b) Polymer structure of methylviologen and naphthol functionalized poly(N-hydroxyethyl acrylamide). ${ }^{189}$

\subsubsection{Cucurbit[n]uril supramolecular nanoparticles stabilized by hydrophobic interactions}

In 2006, Kim and coworkers reported the formation of vesicles using supramolecular complexes of $\mathrm{CB}[6] .{ }^{191}$ This required the covalent modification of (allyloxy) ${ }_{12} \mathrm{CB}[6]$ with 2-(2-(2-methoxyethoxy)ethoxy)ethanethiol to form an amphiphilic host. In general, chemical modification of $C B$ is not trivial, and heavy synthetic and purification efforts are required to obtain pure $\mathrm{CB}[\mathrm{n}]$ homologues with functional groups at the periphery. ${ }^{192}$ Sonication of the modified CB[6] in aqueous environment led to vesicles of 30-1000 nm, as observed by light scattering and TEM. More monodisperse SNPS were prepared by chemical cross-linking of (allyloxy) ${ }_{12} \mathrm{CB}[6]$ with 6-mercaptohexanol ${ }^{193}$ and dithiols ${ }^{194,195}$ followed by sonication induced vesicle formation. The size of the covalently bound nanocapsules depended on the reaction medium and the length of the cross-linker. For 
example, capsules prepared in methanol ranged between $50-110 \mathrm{~nm}$ depending on the length of the dithiol. These types of responsive nanocapsules have high potential in biomedical applications, as the hollow core and the supramolecular shell enables encapsulation of active compounds and decoration with different cell receptors, respectively. ${ }^{196-198}$

Zhou and coworkers have reported the formation of supramolecular vesicles by hostguest complexation of $\mathrm{CB}[6]$ and $N, N$ 'hexamethylene-bis(1-octyl-4-carbamoyl pyridinium bromide) (HBPB-8) in water. ${ }^{199}$ After host-guest complexation, the complexes formed vesicles, in which the hydrophilic $\mathrm{CB}[6]$ host molecules face the aqueous domains, whereas the hydrophobic tails of the pseudorotaxane are directed into the bilayer. Using a modified guest molecule and $\mathrm{CB}[7]$ to form a pseudorotaxane, supramolecular aggregate transformation from micelles to large vesicles was induced by subsequent addition of $\mathrm{CB}[6]{ }^{200}$

The formation of CB-based supramolecular amphiphiles mediated by the ternary hostguest-guest interaction of $\mathrm{CB}[8]$, 2,6-dihydroxynaphthalene and alkylated $\mathrm{MV}$ was reported in 2002. ${ }^{201}$ Initiated by the heteroternary complexation, the formation of supramolecular vesicles was induced by hydrophobic interactions between the alkyl chains. In the absence of $\mathrm{CB}[8]$ and 2,6-dihydroxynaphthalene, micelle formation of the MV derivative was observed. The structure of the MV derivative strongly affected the appearance of the host-guest stabilized vesicles. Relatively monodisperse vesicles of 20 $\mathrm{nm}$ were formed by MV functionalized with dodecyl, whereas vesicles ranging in size from $20 \mathrm{~nm}$ to $1.2 \mu \mathrm{m}$ were observed for structures formed by MV-hexadecyl in the presence of $\mathrm{CB}[8]$ and 2,6-dihydroxynaphthalene.

Using the same CB-induced charge-transfer motif, Scherman and coworkers reported the formation of supramolecular block copolymers, which self-assemble in water into larger supramolecular aggregates. ${ }^{202,203}$ MV-modified poly( $N$-isopropylacrylamide) (PNIPAAm) and Np-modified poly(dimethylaminoethylmethacrylate) (PDMAEMA), provided thermoand $\mathrm{pH}$-responsive supramolecular guest molecules, respectively, which gave supramolecular micelles upon addition of $\mathrm{CB}[8] .{ }^{203}$ The hydrophobic core of the aggregates enabled the encapsulation of DOX. Triggered release of the anti-cancer drug was enabled upon addition of the competing Ad guest molecule, a decrease in temperature or a decrease in $\mathrm{pH}$, thus inhibiting the proliferation of HeLa cells.

Peptide amphiphiles were formed using the ternary complex of pyrene, $\mathrm{MV}$, and $\mathrm{CB}[8]$ as the interaction motif. ${ }^{204,205}$ Supramolecular peptide vesicles with sizes of about $200 \mathrm{~nm}$ were formed from a pyrene-imidazole conjugated peptide and $\mathrm{CB}[8]$ in the presence of MV-octadecyl ${ }^{204}$ or MV-PNIPAAm. ${ }^{205}$ Next to guest integration, the functionalization of the 
hydrophilic peptide with pyrene-imidazole enabled the integration of a fluorescent marker which allowed visualization of the formation and rupture of the vesicles. In contrast to the vesicles formed by MV-octadecyl, the use of MV-PNIPAAm required heating to $37^{\circ} \mathrm{C}$ to render the polymer hydrophobic. Selective dissociation of the vesicles was achieved by addition of competing guest molecules, naphthalene and adamantantylamine. The peptide vesicle formed by MV-PNIPAAm enabled the encapsulation of basic fibroblast growth, which protected the protein against denaturation.

\subsubsection{Cucurbit[n]uril supramolecular nanoparticles stabilized by electrostatic interactions}

Kim, Park and coworkers have reported the formation of non-toxic self-assembled gene delivery vehicles based on electrostatic interactions and $\mathrm{CB}[6]$ inclusion chemistry. ${ }^{206}$ Hereto, different generations of cationic diaminobutane (DAB)-grafted poly(propyleneimine) dendrimers were synthesized. Two DAB head groups constitute a homoternary inclusion complex with $\mathrm{CB}[6]$. The cationic character of the dendrimer led to polyplex formation in the presence of DNA. The size of the observed polyplexes depended strongly on the addition of the supramolecular host components. In the absence of $C B[6]$, polyplexes of $160 \mathrm{~nm}$ were observed for the $D A B$ dendrimer with of DNA. By addition of $\mathrm{CB}[6]$, the size of the electrostatically stabilized SNPs increased from 300 to $420 \mathrm{~nm}$, depending on the amount of $\mathrm{CB}[6]$ inclusion cross-links. Furthermore, molecular dynamic simulation showed that the host-guest stabilized SNPs have a more rigid shell than the polyplexes.

\subsection{Conclusions}

Supramolecular nanoparticles represent a highly attractive platform for the formation of stable, yet reversible nanovectors. Induced by different non-covalent interactions, a broad variety of self-assembled structures has been reported in the literature over the last 20 years. The core and shell of the formed SNPs were modified to allow a variety of functional molecules to be integrated for biomolecular recognition, imaging, drug delivery, and photothermal treatment. The chemical flexibility of SNPs enables selective decoration of the particle shell by cell targeting receptors and the integration of responsive supramolecular clues for triggered SNP disassembly. Full realization of their potential requires better understanding of their assembly thermodynamics and assembly/disassembly mechanisms. Apart from their constituting supramolecular interaction motif, other structure determining parameters often remain obscure. Hence, understanding the formation, stability and disassembly of SNPs requires more efforts and will contribute to the design of functional and biomedically relevant nanodevices. 


\subsection{References}

1. T. M. Allen and P. R. Cullis, Science, 2004, 303, 1818-1822.

2. M. Ferrari, Nature Review Cancer, 2005, 5, 161-171.

3. M. De, P. S. Ghosh and V. M. Rotello, Advanced Materials, 2008, 20, 4225-4241.

4. N. Sanvicens and M. P. Marco, Trends in Biotechnology, 2008, 26, 425-433.

5. E. C. Wang and A. Z. Wang, Integrative Biology, 2014, 6, 9-26.

6. O. C. Farokhzad and R. Langer, ACS Nano, 2009, 3, 16-20.

7. M. Khan, Z. Y. Ong, N. Wiradharma, A. B. E. Attia and Y.-Y. Yang, Advanced Healthcare Materials, 2012, 1, 373-392.

8. L. Brannon-Peppas and J. O. Blanchette, Advanced Drug Delivery Reviews, 2012, 64, Supplement, 206-212.

9. S. Mura, J. Nicolas and P. Couvreur, Nature Materials, 2013, 12, 991-1003.

10. J. Cheon and J.-H. Lee, Accounts of Chemical Research, 2008, 41, 1630-1640.

11. R. Weissleder, M. Nahrendorf and M. J. Pittet, Nature Materials, 2014, 13, 125-138.

12. S. M. Janib, A. S. Moses and J. A. MacKay, Advanced Drug Delivery Reviews, 2010, 62, 1052-1063.

13. J. Xie, S. Lee and X. Chen, Advanced Drug Delivery Reviews, 2010, 62, 1064-1079.

14. Y. Liu and N. Zhang, Biomaterials, 2012, 33, 5363-5375.

15. A. M. Nyström and K. L. Wooley, Accounts of Chemical Research, 2011, 44, 969-978.

16. S. Rose, A. Prevoteau, P. Elziere, D. Hourdet, A. Marcellan and L. Leibler, Nature, 2014, 505, 382-385.

17. M. E. Davis, MRS Bulletin, 2012, 37, 828-835.

18. G. M. Whitesides and B. Grzybowski, Science, 2002, 295, 2418-2421.

19. G. M. Whitesides, J. P. Mathias and C. T. Seto, Science, 1991, 254, 1312-1319.

20. G. V. Oshovsky, D. N. Reinhoudt and W. Verboom, Angewandte Chemie International Edition, 2007, 46, 2366-2393.

21. J.-H. Ryu, D.-J. Hong and M. Lee, Chemical Communications, 2008, 1043-1054.

22. J. M. Zayed, N. Nouvel, U. Rauwald and O. A. Scherman, Chemical Society Reviews, 2010, 39, 2806-2816.

23. E. A. Kataev and C. Müller, Tetrahedron, 2014, 70, 137-167.

24. S. Mann, Nature Materials, 2009, 8, 781-792.

25. J. D. Badjić, A. Nelson, S. J. Cantrill, W. B. Turnbull and J. F. Stoddart, Accounts of Chemical Research, 2005, 38, 723-732.

26. J. Huskens, Current Opinion in Chemical Biology, 2006, 10, 537-543.

27. A. Barnard and D. K. Smith, Angewandte Chemie International Edition, 2012, 51, 65726581.

28. G. R. Desiraju, Accounts of Chemical Research, 1996, 29, 441-449.

29. A. Mulder, J. Huskens and D. N. Reinhoudt, Organic \& Biomolecular Chemistry, 2004, 2, 3409-3424.

30. M. Mammen, S.-K. Choi and G. M. Whitesides, Angewandte Chemie International Edition, 1998, 37, 2754-2794.

31. J. Huskens, A. Mulder, T. Auletta, C. A. Nijhuis, M. J. W. Ludden and D. N. Reinhoudt, Journal of the American Chemical Society, 2004, 126, 6784-6797.

32. W. Kuhn, Kolloid-Zeitschrift, 1934, 68, 2-15. 
33. C. Fasting, C. A. Schalley, M. Weber, O. Seitz, S. Hecht, B. Koksch, J. Dernedde, C. Graf, E.-W. Knapp and R. Haag, Angewandte Chemie International Edition, 2012, 51, 1047210498.

34. A. B. Descalzo, R. Martínez-Máñez, F. Sancenon, K. Hoffmann and K. Rurack, Angewandte Chemie International Edition, 2006, 45, 5924-5948.

35. M. Grzelczak, J. Vermant, E. M. Furst and L. M. Liz-Marzán, ACS Nano, 2010, 4, 35913605.

36. D. Grosso, F. Ribot, C. Boissiere and C. Sanchez, Chemical Society Reviews, 2011, 40, 829-848.

37. H. J. Kim, M. H. Lee, L. Mutihac, J. Vicens and J. S. Kim, Chemical Society Reviews, 2012, 41, 1173-1190.

38. Y. Ofir, B. Samanta and V. M. Rotello, Chemical Society Reviews, 2008, 37, 1814-1825.

39. R. Mout, D. F. Moyano, S. Rana and V. M. Rotello, Chemical Society Reviews, 2012, 41, 2539-2544.

40. C. S. Kim, B. Duncan, B. Creran and V. M. Rotello, Nano Today, 2013, 8, 439-447.

41. C. Wang, Z. Wang and X. Zhang, Accounts of Chemical Research, 2012, 45, 608-618.

42. A. Sorrenti, O. Illa and R. M. Ortuno, Chemical Society Reviews, 2013, 42, 8200-8219.

43. M. L. Immordino, F. Dosio and L. Cattel, International Journal of Nanomedicine, 2006, 1, 297.

44. H.-I. Chang and M.-K. Yeh, International Journal of Nanomedicine, 2012, 7, 49.

45. D. E. Discher and F. Ahmed, Annual Reviews of Biomedical Engineering, 2006, 8, 323341.

46. J. S. Lee and J. Feijen, Journal of Controlled Release, 2012, 161, 473-483.

47. T. Shimizu, M. Masuda and H. Minamikawa, Chemical Reviews, 2005, 105, 1401-1444.

48. X.-M. Chen and G.-F. Liu, Chemistry - A European Journal, 2002, 8, 4811-4817.

49. Y. Wang, H. Xu and X. Zhang, Advanced Materials, 2009, 21, 2849-2864.

50. D. C. Sherrington and K. A. Taskinen, Chemical Society Reviews, 2001, 30, 83-93.

51. G. M. Whitesides, E. E. Simanek, J. P. Mathias, C. T. Seto, D. Chin, M. Mammen and D. M. Gordon, Accounts of chemical research, 1995, 28, 37-44.

52. A. K. Boal, F. Ilhan, J. E. DeRouchey, T. Thurn-Albrecht, T. P. Russell and V. M. Rotello, Nature, 2000, 404, 746-748.

53. C. R. v. d. Brom, P. Rudolf, T. T. M. Palstra and B. Hessen, Chemical Communications, 2007, 4922-4924.

54. B. L. Frankamp, O. Uzun, F. Ilhan, A. K. Boal and V. M. Rotello, Journal of the American Chemical Society, 2002, 124, 892-893.

55. O. Uzun, B. L. Frankamp, A. Sanyal and V. M. Rotello, Chemistry of Materials, 2006, 18, 5404-5409.

56. F. Ilhan, T. H. Galow, M. Gray, G. Clavier and V. M. Rotello, Journal of the American Chemical Society, 2000, 122, 5895-5896.

57. U. Drechsler, R. J. Thibault and V. M. Rotello, Macromolecules, 2002, 35, 9621-9623.

58. O. Uzun, A. Sanyal, H. Nakade, R. J. Thibault and V. M. Rotello, Journal of the American Chemical Society, 2004, 126, 14773-14777.

59. O. Uzun, A. Sanyal, Y. Jeong and V. M. Rotello, Macromolecular Bioscience, 2010, 10, 481-487.

60. G. Decher, Science, 1997, 277, 1232-1237. 
61. K. Ariga, Y. M. Lvov, K. Kawakami, Q. Ji and J. P. Hill, Advanced Drug Delivery Reviews, 2011, 63, 762-771.

62. G. K. Such, A. P. R. Johnston and F. Caruso, Chemical Society Reviews, 2011, 40, 19-29.

63. P. Felgner, Y. Barenholz, J. Behr, S. Cheng, P. Cullis, L. Huang, J. Jessee, L. Seymour, F. Szoka and A. Thierry, Human Gene Therapy, 1997, 8, 511.

64. C. A. Fernandez and K. G. Rice, Molecular Pharmaceutics, 2009, 6, 1277-1289.

65. H. Tian, J. Chen and X. Chen, Small, 2013, 9, 2034-2044.

66. S. D. Li and L. Huang, Gene Therapie, 2006, 13, 1313-1319.

67. J. Hamman, Marine Drugs, 2009, 8, 1305-1322.

68. A. J. Friedman, J. Phan, D. O. Schairer, J. Champer, M. Qin, A. Pirouz, K. Blecher-Paz, A. Oren, P. T. Liu, R. L. Modlin and J. Kim, Journal of Investigative Dermatologie, 2013, 133, 1231-1239.

69. A. Grenha, M. E. Gomes, M. Rodrigues, V. E. Santo, J. F. Mano, N. M. Neves and R. L. Reis, Journal of Biomedical Materials Research Part A, 2010, 92A, 1265-1272.

70. N. Duceppe and M. Tabrizian, Biomaterials, 2009, 30, 2625-2631.

71. F. Bigucci, B. Luppi, T. Cerchiara, M. Sorrenti, G. Bettinetti, L. Rodriguez and V. Zecchi, European Journal of Pharmaceutical Sciences, 2008, 35, 435-441.

72. N. P. Birch and J. D. Schiffman, Langmuir, 2014, 30, 3441-3447.

73. S. Argin-Soysal, P. Kofinas and Y. M. Lo, Food Hydrocolloids, 2009, 23, 202-209.

74. C.-H. Chu, H. Kumagai and K. Nakamura, Journal of Applied Polymer Science, 1996, 60, 1041-1047.

75. V. G. M. Naidu, K. Madhusudhana, R. B. Sashidhar, S. Ramakrishna, R. K. Khar, F. J. Ahmed and P. V. Diwan, Carbohydrate Polymers, 2009, 76, 464-471.

76. Shelly, M. Ahuja and A. Kumar, International Journal of Biological Macromolecules, 2013, 61, 411-415.

77. C. Schatz, A. Domard, C. Viton, C. Pichot and T. Delair, Biomacromolecules, 2004, 5, $1882-1892$.

78. A. Drogoz, L. David, C. Rochas, A. Domard and T. Delair, Langmuir, 2007, 23, 1095010958.

79. S. Shu, L. Sun, X. Zhang, Z. Wu, Z. Wang and C. Li, Journal of Nanoparticle Research, 2011, 13, 3657-3670.

80. J.-H. Kim, S. Lee, K. Kim, H. Jeon, R.-W. Park, I.-S. Kim, K. Choi and I. C. Kwon, Chemical Communications, 2007, 1346-1348.

81. J.-H. Kim, S. Lee, K. Park, H. Y. Nam, S. Y. Jang, I. Youn, K. Kim, H. Jeon, R.-W. Park, I.-

S. Kim, K. Choi and I. C. Kwon, Angewandte Chemie International Edition, 2007, 46, 5779-5782.

82. J. W. Steed and J. L. Atwood, Supramolecular Chemistry, John Wiley \& Sons, 2009.

83. K. Uekama, F. Hirayama and T. Irie, Chemical Reviews, 1998, 98, 2045-2076.

84. J. Zhang and P. X. Ma, Advanced Drug Delivery Reviews, 2013, 65, 1215-1233.

85. F. van de Manakker, T. Vermonden, C. F. van Nostrum and W. E. Hennink, Biomacromolecules, 2009, 10, 3157-3175.

86. H. Gonzalez, S. J. Hwang and M. E. Davis, Bioconjugate Chemistry, 1999, 10, 1068-1074.

87. S. J. Hwang, N. C. Bellocq and M. E. Davis, Bioconjugate Chemistry, 2001, 12, 280-290.

88. S. Hwang and M. Davis, Current Opinion in Molecular Therapeutics, 2001, 3, 183-191.

89. T. M. Reineke and M. E. Davis, Bioconjugate Chemistry, 2002, 14, 247-254. 
90. T. M. Reineke and M. E. Davis, Bioconjugate Chemistry, 2002, 14, 255-261.

91. S. H. Pun and M. E. Davis, Bioconjugate Chemistry, 2002, 13, 630-639.

92. S. Mishra, P. Webster and M. E. Davis, European Journal of Cell Biology, 2004, 83, 97111.

93. S. H. Pun, F. Tack, N. C. Bellocq, J. Cheng, B. H. Grubbs, G. S. Jensen, M. E. Davis, M. Brewster, M. Janicot, B. Janssens, W. Floren and A. Bakker, Cancer Biology \& Therapy, 2004, 3, 641-650.

94. N. C. Bellocq, S. H. Pun, G. S. Jensen and M. E. Davis, Bioconjugate Chemistry, 2003, 14, 1122-1132.

95. D. W. Bartlett and M. E. Davis, Bioconjugate Chemistry, 2007, 18, 456-468.

96. S. Mishra, J. D. Heidel, P. Webster and M. E. Davis, Journal of Controlled Release, 2006, 116, 179-191.

97. D. W. Bartlett, H. Su, I. J. Hildebrandt, W. A. Weber and M. E. Davis, Proceedings of the National Academy of Sciences, 2007, 104, 15549-15554.

98. J. D. Heidel, Z. Yu, J. Y.-C. Liu, S. M. Rele, Y. Liang, R. K. Zeidan, D. J. Kornbrust and M.

E. Davis, Proceedings of the National Academy of Sciences, 2007, 104, 5715-5721.

99. M. E. Davis, J. E. Zuckerman, C. H. J. Choi, D. Seligson, A. Tolcher, C. A. Alabi, Y. Yen, J. D. Heidel and A. Ribas, Nature, 2010, 464, 1067-1070.

100. J. E. Zuckerman, C. H. J. Choi, H. Han and M. E. Davis, Proceedings of the National Academy of Sciences, 2012, 109, 3137-3142.

101. Y. Ping, C. Liu, Z. Zhang, K. L. Liu, J. Chen and J. Li, Biomaterials, 2011, 32, 8328-8341.

102. Y. Ping, Q. Hu, G. Tang and J. Li, Biomaterials, 2013, 34, 6482-6494.

103. W. Deng, J. Chen, A. Kulkarni and D. H. Thompson, Soft Matter, 2012, 8, 5843-5846.

104. A. Kulkarni, K. Defrees, S.-H. Hyun and D. H. Thompson, Journal of the American Chemical Society, 2012, 134, 7596-7599.

105. A. Kulkarni, W. Deng, S.-h. Hyun and D. H. Thompson, Bioconjugate Chemistry, 2012, 23, 933-940.

106. A. Kulkarni, V. Badwaik, K. DeFrees, R. A. Schuldt, D. S. Gunasekera, C. Powers, A. Vlahu, R. VerHeul and D. H. Thompson, Biomacromolecules, 2013, 15, 12-19.

107. A. Kulkarni, R. VerHeul, K. DeFrees, C. J. Collins, R. A. Schuldt, A. Vlahu and D. H. Thompson, Biomaterials Science, 2013, 1, 1029-1033.

108. C. Galant, C. Amiel and L. Auvray, Macromolecular Bioscience, 2005, 5, 1057-1065.

109. V. Burckbuchler, V. Wintgens, C. Leborgne, S. Lecomte, N. Leygue, D. Scherman, A. Kichler and C. Amiel, Bioconjugate Chemistry, 2008, 19, 2311-2320.

110. M. E. Davis, Advanced Drug Delivery Reviews, 2009, 61, 1189-1192.

111. J. Cheng, K. T. Khin, G. S. Jensen, A. Liu and M. E. Davis, Bioconjugate Chemistry, 2003, 14, 1007-1017.

112. T. Schluep, J. Hwang, I. J. Hildebrandt, J. Czernin, C. H. J. Choi, C. A. Alabi, B. C. Mack and M. E. Davis, Proceedings of the National Academy of Sciences, 2009, 106, 1139411399.

113. G. Weiss, J. Chao, J. Neidhart, R. Ramanathan, D. Bassett, J. Neidhart, C. Choi, W. Chow, V. Chung, S. Forman, E. Garmey, J. Hwang, D. L. Kalinoski, M. Koczywas, J. Longmate, R. Melton, R. Morgan, J. Oliver, J. Peterkin, J. Ryan, T. Schluep, T. Synold, P. Twardowski, M. Davis and Y. Yen, Investigational New Drugs, 2013, 31, 986-1000. 
114. S. Eliasof, D. Lazarus, C. G. Peters, R. I. Case, R. O. Cole, J. Hwang, T. Schluep, J. Chao, J. Lin, Y. Yen, H. Han, D. T. Wiley, J. E. Zuckerman and M. E. Davis, Proceedings of the National Academy of Sciences, 2013, 110, 15127-15132.

115. T. Schluep, P. Gunawan, L. Ma, G. S. Jensen, J. Duringer, S. Hinton, W. Richter and J. Hwang, Clinical Cancer Research, 2009, 15, 181-189.

116. X. Lu, Q.-Q. Wang, F.-J. Xu, G.-P. Tang and W.-T. Yang, Biomaterials, 2011, 32, 48494856.

117. Y. Cao, B. Wang, Y. Wang and D. Lou, RSC Advances, 2014, 4, 30430-30439.

118. J. Wang and M. Jiang, Journal of the American Chemical Society, 2006, 128, 3703-3708.

119. J. Zhang and P. X. Ma, Angewandte Chemie International Edition, 2009, 48, 964-968.

120. J. Zhang, K. Ellsworth and P. X. Ma, Macromolecular Rapid Communications, 2012, 33, 664-671.

121. J. Zhang and P. X. Ma, Polymer, 2011, 52, 4928-4937.

122. J. Zhang, K. Feng, M. Cuddihy, N. A. Kotov and P. X. Ma, Soft Matter, 2010, 6, 610-617.

123. J. Zhang, K. Ellsworth and P. X. Ma, Journal of Controlled Release, 2010, 145, 116-123.

124. R. Gref, C. Amiel, K. Molinard, S. Daoud-Mahammed, B. Sébille, B. Gillet, J.-C. Beloeil, C. Ringard, V. Rosilio, J. Poupaert and P. Couvreur, Journal of Controlled Release, 2006, 111, 316-324.

125. E. Battistini, E. Gianolio, R. Gref, P. Couvreur, S. Fuzerova, M. Othman, S. Aime, B. Badet and P. Durand, Chemistry - A European Journal, 2008, 14, 4551-4561.

126. A.-M. Layre, V. Wintgens, N.-M. Gosselet, F. Dalmas and C. Amiel, European Polymer Journal, 2009, 45, 3016-3026.

127. V. Wintgens, T. T. Nielsen, K. L. Larsen and C. Amiel, Macromolecular Bioscience, 2011, $11,1254-1263$.

128. H. Wang, S. Wang, H. Su, K.-J. Chen, A. L. Armijo, W.-Y. Lin, Y. Wang, J. Sun, K.-i. Kamei, J. Czernin, C. G. Radu and H.-R. Tseng, Angewandte Chemie International Edition, 2009, 48, 4344-4348.

129. K. Liu, H. Wang, K.-J. Chen, F. Guo, W.-Y. Lin, Y.-C. Chen, D. L. Phung, H.-R. Tseng and C. K.-F. Shen, Nanotechnology, 2010, 21, 445603.

130. H. Wang, K. Liu, K.-J. Chen, Y. Lu, S. Wang, W.-Y. Lin, F. Guo, K.-i. Kamei, Y.-C. Chen, M. Ohashi, M. Wang, M. A. Garcia, X.-Z. Zhao, C. K. F. Shen and H.-R. Tseng, ACS Nano, 2010, 4, 6235-6243.

131. K.-J. Chen, S. M. Wolahan, H. Wang, C.-H. Hsu, H.-W. Chang, A. Durazo, L.-P. Hwang, M. A. Garcia, Z. K. Jiang, L. Wu, Y.-Y. Lin and H.-R. Tseng, Biomaterials, 2011, 32, 21602165.

132. H. Wang, K.-J. Chen, S. Wang, M. Ohashi, K.-i. Kamei, J. Sun, J. H. Ha, K. Liu and H.-R. Tseng, Chemical Communications, 2010, 46, 1851-1853.

133. Y. Liu, H. Wang, K.-i. Kamei, M. Yan, K.-J. Chen, Q. Yuan, L. Shi, Y. Lu and H.-R. Tseng, Angewandte Chemie International Edition, 2011, 50, 3058-3062.

134. K.-J. Chen, L. Tang, M. A. Garcia, H. Wang, H. Lu, W.-Y. Lin, S. Hou, Q. Yin, C. K. F. Shen, J. Cheng and H.-R. Tseng, Biomaterials, 2012, 33, 1162-1169.

135. S. Wang, K.-J. Chen, T.-H. Wu, H. Wang, W.-Y. Lin, M. Ohashi, P.-Y. Chiou and H.-R. Tseng, Angewandte Chemie International Edition, 2010, 49, 3777-3781. 
136. J.-H. Lee, K.-J. Chen, S.-H. Noh, M. A. Garcia, H. Wang, W.-Y. Lin, H. Jeong, B. J. Kong, D. B. Stout, J. Cheon and H.-R. Tseng, Angewandte Chemie International Edition, 2013, $52,4384-4388$.

137. L. Grana Suarez, W. Verboom and J. Huskens, Chemical Communications, 2014, 50, 7280-7282.

138. Z. Wang, Z. Feng and C. Gao, Chemistry of Materials, 2008, 20, 4194-4199.

139. W. Xiao, W.-H. Chen, J. Zhang, C. Li, R.-X. Zhuo and X.-Z. Zhang, Journal of Physical Chemistry B, 2011, 115, 13796-13802.

140. P. Zhang, L. Chang-Chun, A. W. Coleman, H. Parrot-Lopez and H. Galons, Tetrahedron Letters, 1991, 32, 2769-2770.

141. O. Häusler and C. C. Müller-Goymann, Starch - Stärke, 1993, 45, 183-187.

142. F. Witte and H. Hoffmann, Journal of Inclusion Phenomena and Molecular Recognition in Chemistry, 1996, 25, 25-28.

143. R. Auzély-Velty, F. Djedaïni-Pilard, S. Désert, B. Perly and T. Zemb, Langmuir, 2000, 16, 3727-3734.

144. B. J. Ravoo and R. Darcy, Angewandte Chemie International Edition, 2000, 39, 43244326.

145. E. Bilensoy and A. A. Hincal, Expert Opinion on Drug Delivery, 2009, 6, 1161-1173.

146. S. K. M. Nalluri, J. Voskuhl, J. B. Bultema, E. J. Boekema and B. J. Ravoo, Angewandte Chemie International Edition, 2011, 50, 9747-9751.

147. L. Jiang, Y. Yan and J. Huang, Advances in Colloid and Interface Science, 2011, 169, 1325.

148. G. Chen and M. Jiang, Chemical Society Reviews, 2011, 40, 2254-2266.

149. J. Zeng, K. Shi, Y. Zhang, X. Sun and B. Zhang, Chemical Communications, 2008, 37533755.

150. H. Liu, Y. Zhang, J. Hu, C. Li and S. Liu, Macromolecular Chemistry and Physics, 2009, 210, 2125-2137.

151. Q. Yan, J. Yuan, Z. Cai, Y. Xin, Y. Kang and Y. Yin, Journal of the American Chemical Society, 2010, 132, 9268-9270.

152. C. Ortiz Mellet, J. M. Benito and J. M. García Fernández, Chemistry - A European Journal, 2010, 16, 6728-6742.

153. S. Srinivasachari, K. M. Fichter and T. M. Reineke, Journal of the American Chemical Society, 2008, 130, 4618-4627.

154. N. Mourtzis, K. Eliadou, C. Aggelidou, V. Sophianopoulou, I. M. Mavridis and K. Yannakopoulou, Organic \& Biomolecular Chemistry, 2007, 5, 125-131.

155. N. Mourtzis, M. Paravatou, I. M. Mavridis, M. L. Roberts and K. Yannakopoulou, Chemistry - A European Journal, 2008, 14, 4188-4200.

156. Q.-D. Hu, H. Fan, Y. Ping, W.-Q. Liang, G.-P. Tang and J. Li, Chemical Communications, 2011, 47, 5572-5574.

157. H. Fan, Q.-D. Hu, F.-J. Xu, W.-Q. Liang, G.-P. Tang and W.-T. Yang, Biomaterials, 2012, 33, 1428-1436.

158. M. Bayrakcı, Ş. Ertul and M. Yilmaz, Journal of Chemical \& Engineering Data, 2011, 57, 233-239.

159. R. Kamada, W. Yoshino, T. Nomura, Y. Chuman, T. Imagawa, T. Suzuki and K. Sakaguchi, Bioorganic \& Medicinal Chemistry Letters, 2010, 20, 4412-4415. 
160. T. Ogoshi, S. Kanai, S. Fujinami, T.-a. Yamagishi and Y. Nakamoto, Journal of the American Chemical Society, 2008, 130, 5022-5023.

161. P. J. Cragg and K. Sharma, Chemical Society Reviews, 2012, 41, 597-607.

162. H. Yan, X. Pan, M. H. Chua, X. Wang, J. Song, Q. Ye, H. Zhou, A. T. Y. Xuan, Y. Liu and J. $\mathrm{Xu}$, RSC Advances, 2014, 4, 10708-10717.

163. H. Zhang, K. T. Nguyen, X. Ma, H. Yan, J. Guo, L. Zhu and Y. Zhao, Organic \& Biomolecular Chemistry, 2013, 11, 2070-2074.

164. K. Helttunen and P. Shahgaldian, New Journal of Chemistry, 2010, 34, 2704-2714.

165. J. J. Michels, J. Huskens, J. F. J. Engbersen and D. N. Reinhoudt, Langmuir, 2000, 16, 4864-4870.

166. Y. Tanaka, M. Miyachi and Y. Kobuke, Angewandte Chemie International Edition, 1999, 38, 504-506.

167. O. Hayashida, K. Mizuki, K. Akagi, A. Matsuo, T. Kanamori, T. Nakai, S. Sando and Y. Aoyama, Journal of the American Chemical Society, 2002, 125, 594-601.

168. Y. Yao, M. Xue, J. Chen, M. Zhang and F. Huang, Journal of the American Chemical Society, 2012, 134, 15712-15715.

169. C. Tu, L. Zhu, P. Li, Y. Chen, Y. Su, D. Yan, X. Zhu and G. Zhou, Chemical Communications, 2011, 47, 6063-6065.

170. Y.-X. Wang, D.-S. Guo, Y. Cao and Y. Liu, RSC Advances, 2013, 3, 8058-8063.

171. D.-S. Guo, K. Wang, Y.-X. Wang and Y. Liu, Journal of the American Chemical Society, 2012, 134, 10244-10250.

172. K. Wang, D.-S. Guo and Y. Liu, Chemistry - A European Journal, 2010, 16, 8006-8011.

173. K. Wang, D.-S. Guo, X. Wang and Y. Liu, ACS Nano, 2011, 5, 2880-2894.

174. J. Bügler, N. A. J. M. Sommerdijk, A. J. W. G. Visser, A. van Hoek, R. J. M. Nolte, J. F. J. Engbersen and D. N. Reinhoudt, Journal of the American Chemical Society, 1998, 121, 28-33.

175. L. Gallego-Yerga, M. Lomazzi, F. Sansone, C. Ortiz Mellet, A. Casnati and J. M. Garcia Fernandez, Chemical Communications, 2014, 50, 7440-7443.

176. H. Zhang, X. Ma, K. T. Nguyen and Y. Zhao, ACS Nano, 2013, 7, 7853-7863.

177. Y. Yao, X. Chi, Y. Zhou and F. Huang, Chemical Science, 2014, 5, 2778-2782.

178. Y. Zhou, Y. Yao and M. Xue, Chemical Communications, 2014, 50, 8040-8042.

179. M. Dudic, A. Colombo, F. Sansone, A. Casnati, G. Donofrio and R. Ungaro, Tetrahedron, 2004, 60, 11613-11618.

180. F. Sansone, M. Dudič, G. Donofrio, C. Rivetti, L. Baldini, A. Casnati, S. Cellai and R. Ungaro, Journal of the American Chemical Society, 2006, 128, 14528-14536.

181. V. Bagnacani, F. Sansone, G. Donofrio, L. Baldini, A. Casnati and R. Ungaro, Organic Letters, 2008, 10, 3953-3956.

182. V. Bagnacani, V. Franceschi, L. Fantuzzi, A. Casnati, G. Donofrio, F. Sansone and R. Ungaro, Bioconjugate Chemistry, 2012, 23, 993-1002.

183. V. Bagnacani, V. Franceschi, M. Bassi, M. Lomazzi, G. Donofrio, F. Sansone, A. Casnati and R. Ungaro, Nature Communications, 2013, 4, 1721.

184. R. V. Rodik, A. S. Klymchenko, N. Jain, S. I. Miroshnichenko, L. Richert, V. I. Kalchenko and Y. Mély, Chemistry - A European Journal, 2011, 17, 5526-5538.

185. J. W. Lee, S. Samal, N. Selvapalam, H.-J. Kim and K. Kim, Accounts of Chemical Research, 2003, 36, 621-630. 
186. J. Lagona, P. Mukhopadhyay, S. Chakrabarti and L. Isaacs, Angewandte Chemie International Edition, 2005, 44, 4844-4870.

187. H.-J. Kim, J. Heo, W. S. Jeon, E. Lee, J. Kim, S. Sakamoto, K. Yamaguchi and K. Kim, Angewandte Chemie International Edition, 2001, 40, 1526-1529.

188. D. Das and O. A. Scherman, Israel Journal of Chemistry, 2011, 51, 537-550.

189. E. A. Appel, J. Dyson, J. del Barrio, Z. Walsh and O. A. Scherman, Angewandte Chemie International Edition, 2012, 51, 4185-4189.

190. E. A. Appel, J. d. Barrio, J. Dyson, L. Isaacs and O. A. Scherman, Chemical Science, 2012, 3, 2278-2281.

191. H.-K. Lee, K. M. Park, Y. J. Jeon, D. Kim, D. H. Oh, H. S. Kim, C. K. Park and K. Kim, Journal of the American Chemical Society, 2005, 127, 5006-5007.

192. K. Kim, N. Selvapalam, Y. H. Ko, K. M. Park, D. Kim and J. Kim, Chemical Society Reviews, 2007, 36, 267-279.

193. K. M. Park, K. Suh, H. Jung, D.-W. Lee, Y. Ahn, J. Kim, K. Baek and K. Kim, Chemical Communications, 2009, 71-73.

194. D. Kim, E. Kim, J. Kim, K. M. Park, K. Baek, M. Jung, Y. H. Ko, W. Sung, H. S. Kim, J. H. Suh, C. G. Park, O. S. Na, D.-k. Lee, K. E. Lee, S. S. Han and K. Kim, Angewandte Chemie, 2007, 119, 3541-3544.

195. D. Kim, E. Kim, J. Lee, S. Hong, W. Sung, N. Lim, C. G. Park and K. Kim, Journal of the American Chemical Society, 2010, 132, 9908-9919.

196. E. Kim, J. Lee, D. Kim, K. E. Lee, S. S. Han, N. Lim, J. Kang, C. G. Park and K. Kim, Chemical Communications, 2009, 1472-1474.

197. K. M. Park, D.-W. Lee, B. Sarkar, H. Jung, J. Kim, Y. H. Ko, K. E. Lee, H. Jeon and K. Kim, Small, 2010, 6, 1430-1441.

198. E. Kim, D. Kim, H. Jung, J. Lee, S. Paul, N. Selvapalam, Y. Yang, N. Lim, C. G. Park and K. Kim, Angewandte Chemie, 2010, 122, 4507-4510.

199. Q. Zhou, H. Wang, T. Gao, Y. Yu, B. Ling, L. Mao, H. Zhang, X. Meng and X. Zhou, Chemical Communications, 2011, 47, 11315-11317.

200. Q. Zhou, D. Gao, J. Liu, X. Sun, L. Zhang, B. Qi, H. Zhang, C. Xia and X. Zhou, Journal of Colloid and Interface Science, 2013, 410, 131-139.

201. Y. J. Jeon, P. K. Bharadwaj, S. Choi, J. W. Lee and K. Kim, Angewandte Chemie International Edition, 2002, 41, 4474-4476.

202. U. Rauwald and O. A. Scherman, Angewandte Chemie International Edition, 2008, 47, 3950-3953.

203. X. J. Loh, J. del Barrio, P. P. C. Toh, T.-C. Lee, D. Jiao, U. Rauwald, E. A. Appel and O. A. Scherman, Biomacromolecules, 2011, 13, 84-91.

204. D. Jiao, J. Geng, X. Loh, D. Das, T. Lee and O. Scherman, Angewandte Chemie International Edition, 2012, 51, 9633-9637.

205. X. J. Loh, J. del Barrio, T.-C. Lee and O. A. Scherman, Chemical Communications, 2014, 50, 3033-3035.

206. Y.-b. Lim, T. Kim, J. W. Lee, S.-m. Kim, H.-J. Kim, K. Kim and J.-s. Park, Bioconjugate Chemistry, 2002, 13, 1181-1185. 


\title{
Chapter 3
}

\section{Size-Tunable Supramolecular Nanoparticles Mediated by Ternary Cucurbit[8]uril Host-Guest Interactions}

\begin{abstract}
Supramolecular nanoparticles (SNPs) stabilized by host-guest interactions are of great interest because of their potential for biomedical applications. The most prominent examples of SNPs in the literature use $\beta$-cyclodextrin-adamantyl host-guest interactions as the interaction motif. In this chapter, we show that cucurbit[8]uril (CB[8])-assisted charge-transfer complexes between naphthol (Np) and methyl viologen (MV) can be used for the formation of size-controlled SNPS. DLS and SEM analyses show that size control of the particles is achieved by tuning the ratio of the multivalent and monovalent $\mathrm{Np}$ derivatives. The formation of these size-tunable SNPs appear to be strongly time and temperature dependent, despite the fast ternary complex formation observed at all temperatures employed. This indicates that SNP formation requires dynamic disassembly and reassembly of the constituting ternary complex units.
\end{abstract}

This chapter is based on: C. Stoffelen and J. Huskens, Chemical Communications, 2013 49, 67406742 


\subsection{Introduction}

Supramolecular chemistry promises the assembly of molecular units into well-defined architectures, via specific non-covalent interactions. ${ }^{1-3}$ Nanoparticles (NPs) possess unique size-dependent properties, that are not observable in bulk materials. ${ }^{4}$ The combination of both features is attractive, since stable, yet reversible hybrid assemblies hold high promises for biomedical applications. ${ }^{5-7}$ External triggers can induce the selective disassembly of supramolecular systems, which leads to responsive drug delivery formulations. ${ }^{8,9}$

Supramolecular nanoparticles (SNPs) can be considered as particles in which multiple copies of different building blocks are brought together by specific noncovalent interactions, giving assemblies that are typically larger than the building blocks themselves. The most well studied SNP, a siRNA delivery system, has been developed by the group of Davis, ${ }^{10,11}$ in which DNA delivery vectors were formed owing to electrostatic interactions between a $\beta$-cyclodextrin (CD)-containing polycation in the presence of negatively charged DNA. Stabilization of these relatively weakly bound particles was achieved by supramolecular host-guest interactions of the CD-containing polycation with a monovalent adamantyl (Ad)-grafted poly(ethylene glycol). ${ }^{12}$ In contrast, NP formation exclusively based on supramolecular CD-Ad interactions has been demonstrated by the group of Tseng, whereby the size of the formed NPs could be altered by varying the ratio of the monovalent and multivalent Ad derivatives. ${ }^{13}$

Although multivalency plays an important role in the dynamic molecular assembly and disassembly processes, ${ }^{14-16}$ the mechanism of the formation and size control of the SNPs remains elusive. Beyond the Ad-CD binding motif, a variety of molecular recognition units are available in supramolecular chemistry, however, none have been used for the preparation of size-tunable SNPs. Thus, a fundamental question arises whether SNPs can be formed using different interaction motifs. Additionally, the expected changes in interaction strength and exchange rate may have a profound influence on the assembly and disassembly process of the SNPs.

Cucurbit[n]urils (CB[n]s) constitute a class of macrocyclic molecules that form inclusion complexes through hydrogen bonds and by hydrophobic and ion-dipole interactions, ${ }^{17,18}$ e.g. when using positively charged guests such as methyl viologen (MV). Moreover, MV and naphthol (Np) can form a charge-transfer (CT) complex, which is included in the cavity of $\mathrm{CB}[8]$ to give a ternary complex with a micromolar affinity. ${ }^{19}$ Utilizing this or similar ternary supramolecular motifs, different nanoparticulate ${ }^{20,21}$ and microstructures ${ }^{22}$ have been reported by the groups of Scherman and Abell. In NP studies, ${ }^{20,21}$ however, the CBs have been used merely to crosslink intramolecularly connected guest moieties attached to a single polymer chain, thus smaller NPs are provided due to the collapse of 
the starting guest-modified polymer. The strong binding affinity of this interaction motif, combined with a radically different kinetics compared to CD complexes, make it an interesting candidate to explore for its potential use for the formation of SNPs and to study their assembly kinetics.

Here, we report a novel strategy for the preparation of size-tunable SNPs in water using $\mathrm{CB}[8]$ as an intermolecular host for MV and Np-containing building blocks, under the formation of a host-guest-assisted CT complex. Scheme 3.1 shows a schematic overview of the proposed SNP assembly. The host-guest recognition between MV-functionalized poly(ethylene imine) (MV-PEI), Np-functionalized poly(amidoamine) dendrimer $\left(\mathrm{Np}_{8}-\right.$ PAMAM) and Np-functionalized poly(ethylene glycol) (Np-PEG; $M_{W} \sim 5000 \mathrm{~g} / \mathrm{mol}$ ) in the presence of $\mathrm{CB}[8]$ leads to SNPs composed of four building blocks and held together by multiple ternary complexes.

a)

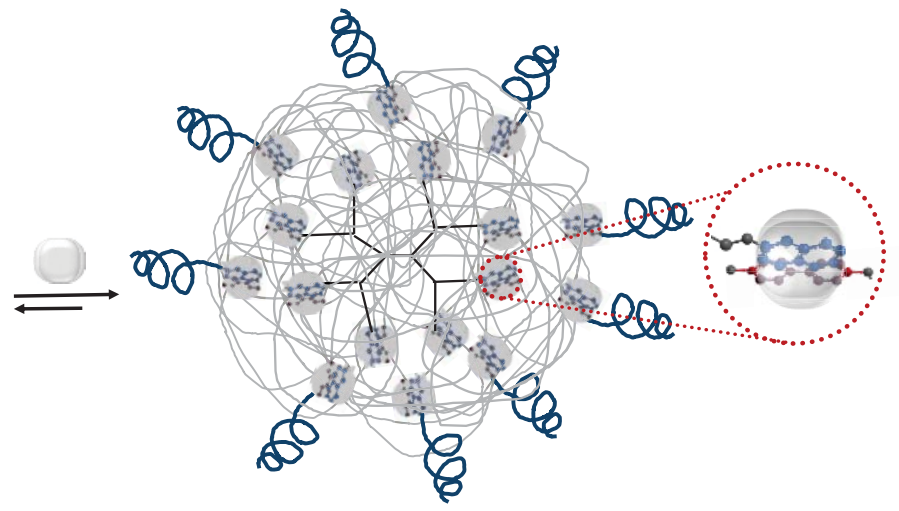

b)

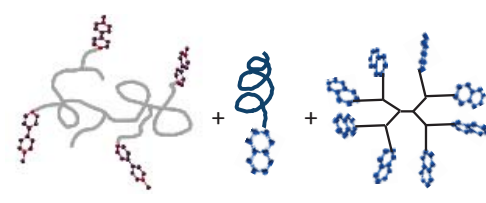

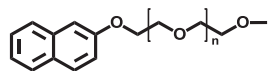

$$
\begin{aligned}
& \mathrm{Np}-\mathrm{PEG}=\text { = } 00.2
\end{aligned}
$$

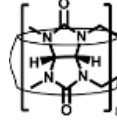

$\mathrm{CB}[8]$
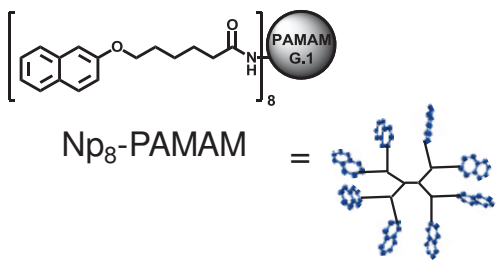

c)
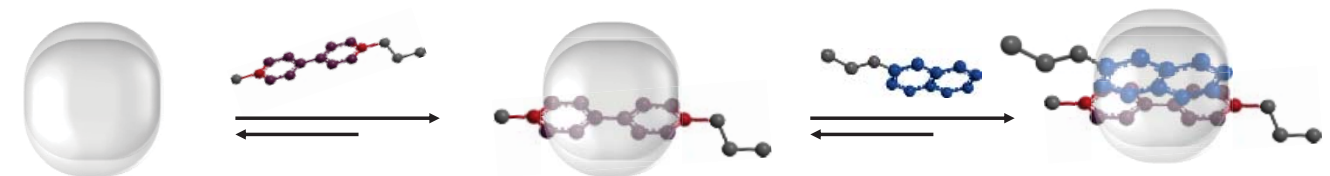

Scheme 3.1: a) Supramolecular nanoparticle (SNP) formation by heteroternary complex formation between cucurbit[8]uril (CB[8]), methyl viologen (MV) and naphthol (Np) moieties. b) The supramolecular building blocks involved in particle formation: methyl viologen-poly(ethylene imine) (MV-PEI), naphthol-poly(ethylene glycol) (Np-PEG), cucurbit[8]uril (CB[8]), and naphthol $8^{-}$ poly(amidoamine) ( $\left.N p_{8}-P A M A M\right)$. c) Ternary complex formation by inclusion of $M V$ in $C B[8]$, followed by inclusion of $\mathrm{Np}$. 


\subsection{Results and Discussion}

\subsubsection{Synthesis of the supramolecular building blocks}

The multivalent building blocks required for the formation of the SNPs are shown in Scheme 3.2. The Np-functionalized PAMAM G1 dendrimer was synthesized by first activating the terminating carboxylic acid group of 2-naphthol-1-hexanoate ${ }^{23}$ with pentafluorophenol. Subsequent reaction of 2-naphthol-1-hexanoic acid pentafluorophenol ester with PAMAM dendrimer $\mathrm{G} 1$ in $\mathrm{CH}_{2} \mathrm{Cl}_{2}$ followed by repeated precipitation in diethyl ether led to $\mathrm{Np}_{8}$-PAMAM. To prepare MV-PEI, poly(ethylene imine) (PEI, $\mathrm{M}_{\mathrm{W}} \sim 10000$ $\mathrm{g} / \mathrm{mol}$ ) was dissolved in dry DMSO and a solution of maleimidohexanoic acid $\mathrm{N}$-hydroxy succinimide ester was added slowly. Reaction with 1-(1-undecyl-11-thiol)-1-methyl-4,4bipyridinium ${ }^{24}$ in water gave the polymer MV-PEI.

a)

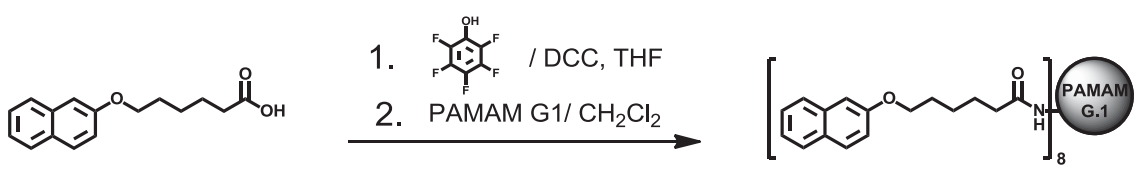

b)

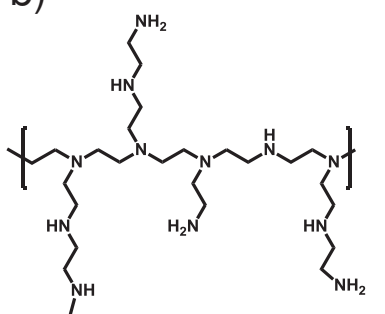

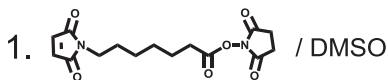<smiles>[Co]=[Co]</smiles>

/ DMSO, buffer pH 6.8

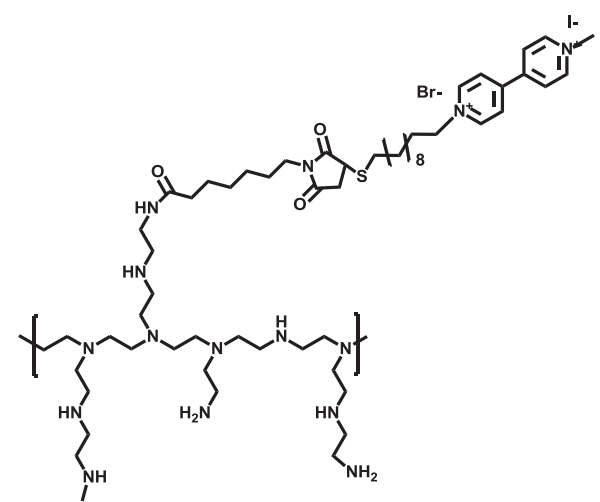

Scheme 3.2: Synthetic routes towards the multivalent building blocks Np ${ }_{-}$-PAMAM (a) and MV-PEI (b) used in this study.

Characterization of the multivalent $\mathrm{Np}_{8}$-PAMAM and the monovalent Np-PEG showed that the reactive binding sites of the parent amino-terminated dendrimer and PEG were fully functionalized with Np guest moieties. The degree of functionalization of the multivalent MV-PEI polymer ( $10 \mathrm{kDa}$ ) was evaluated by UV/Vis spectroscopy, microcalorimetry and ${ }^{1} \mathrm{H}-\mathrm{NMR}$, and all techniques showed each polymer chain to be functionalized with, on average, four MV guest moieties.

\subsubsection{Supramolecular nanoparticle formation in aqueous solution}

Upon addition of aqueous MV-PEI to a previously prepared aqueous solution of $\mathrm{CB}[8]$, $\mathrm{Np}$-PEG and $\mathrm{Np}_{8}$-PAMAM, SNPs were formed using a $0.67 \mu \mathrm{M}$ concentration of each of the three molecular recognition groups (CB[8], MV and Np). The concentrations of Np-PEG and $\mathrm{Np}_{8}$-PAMAM were varied in different ratios, while keeping the total $\mathrm{Np}$ concentration 
constant, in order to study the influence of the multivalent-monovalent competition on the SNP size. Thereby, the resulting intrinsic ratio of the three functional host-guest recognition units $\mathrm{CB}[8]: \mathrm{MV}$ :Np was kept constant and in a 1:1:1 ratio in accordance with the stoichiometry of the basic ternary complex motif. After 2 days, particle formation was confirmed by dynamic light scattering (DLS) and scanning electron microscopy (SEM). As visible in Figure 3.1a, utilizing $20 \% \mathrm{~Np}$ derived from $\mathrm{Np}_{8}$-PAMAM, SNPs with an average hydrodynamic diameter of $85 \pm 15 \mathrm{~nm}$ were observed by DLS. In good agreement, SEM (Figure 3.1b) showed particles with an average diameter of $74 \pm 13 \mathrm{~nm}$.

a)

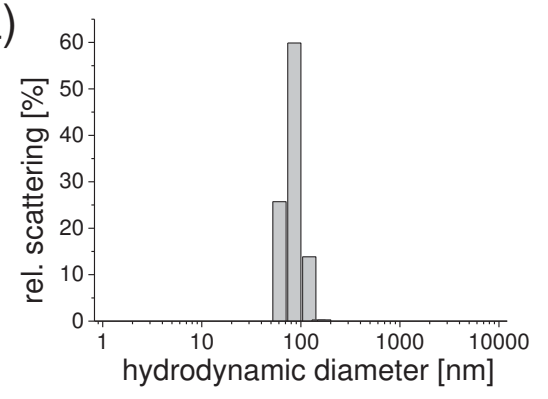

b)

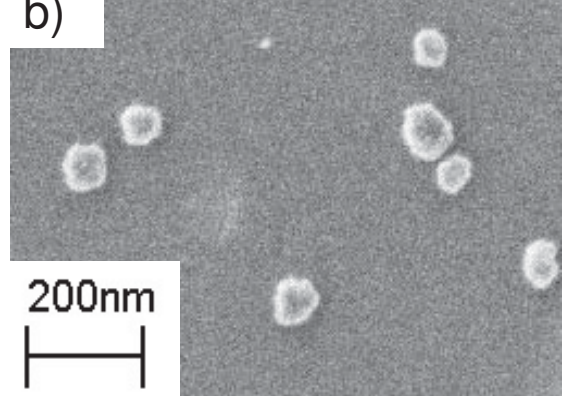

Figure 3.1: Characterization of CB-mediated SNPS prepared using CB[8]:MV:Np $=1: 1: 1$, with 20\% $\mathrm{Np}$ derived from $\mathrm{NP}_{8}$-PAMAM: a) DLS data and b) SEM image.

To evaluate whether the SNP formation is based on the selective host-guest interaction of the different supramolecular building blocks, the formation of the CT complex was studied. An increase in UV/Vis absorbance (Figure 3.2a) as well as a decrease in fluorescence intensity (Figure 3.2b) indicates that the SNPs are assembled in aqueous solution as a result of formation of a ternary supramolecular complex. Congruently, no SNPs were formed in the absence of MV-PEI or $\mathrm{CB}[8]$, or upon addition of $\mathrm{CB}$ [7] instead of $\mathrm{CB}[8]$. The dicationic MV moiety is required to enable inclusion of the neutral $\mathrm{Np}$ inside the cavity of the $\mathrm{CB}[8]$ host, hence no CT complex is formed in the absence of MV. Additionally, intermolecular crosslinking between the MV and Np derivatives cannot occur in the presence of $\mathrm{CB}[7]$; its cavity is too small to host both guest moieties.
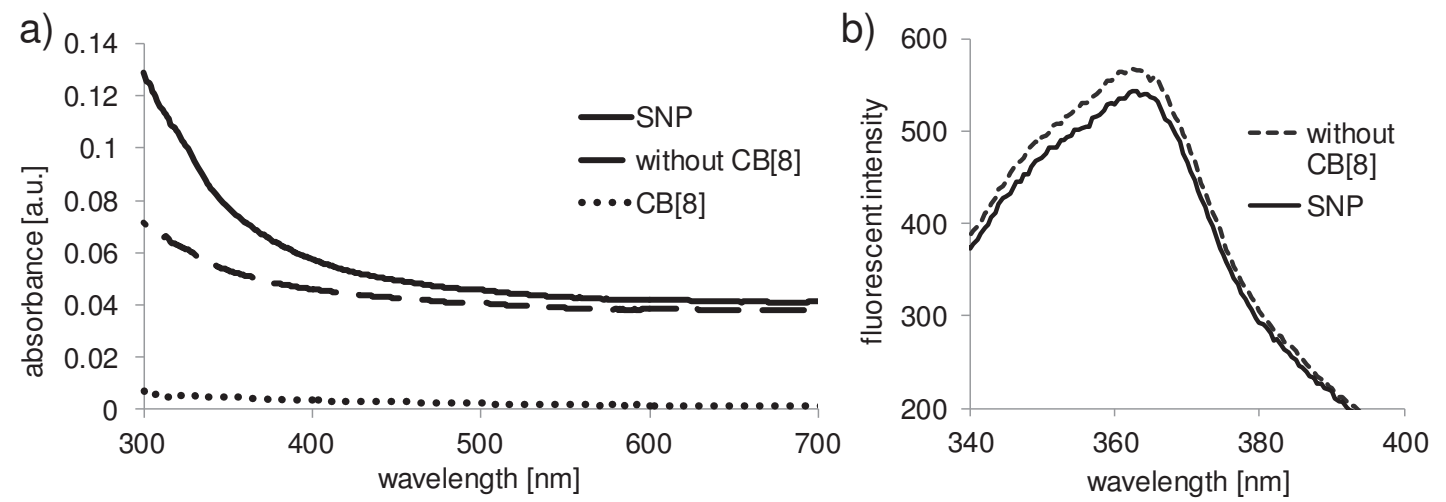

Figure 3.2: a) UV/Vis absorption spectra of an SNP solution in the presence of CB[8] (solid line), an analogous sample in the absence of CB[8] (dashed line) and solely CB[8] (dotted line). b) Fluorescence spectra of the SNP formulation before (dashed line) and after addition of CB[8] (solid line). 
Remarkably, a fluorescence decrease was observed directly after mixing the different supramolecular building blocks at room temperature, whereas consistent SNP formation is only detectable after $48 \mathrm{~h}$. This led to the presumption that the supramolecular hostguest interactions, forming the $\mathrm{CB}[8]$-assisted ternary complexes, are established immediately after mixing the components. Initially, undefined, kinetically trapped structures are formed which slowly reassemble into stable SNPs by exchange of the guest moieties via (slow) dissociation and (fast) reassociation. In order to monitor the assembly behavior of the supramolecular building blocks in more detail, time-dependent DLS measurements were carried out at different temperatures. As seen in Figure 3.3, the apparent sizes observed by DLS using SNP formulations containing $25 \% \mathrm{~Np}$, varied within time, and the extent of variations in SNP size depended on the temperature. At room temperature, the observed amplitude of the SNP size variation was very high and the measurements were not consistent. In contrast, SNP assembly occurred faster at elevated temperatures. Samples kept at $40^{\circ} \mathrm{C}$ showed distinct particle formation and consistent DLS measurements already $2 \mathrm{~h}$ after sample preparation, and a stable SNP size (96 $\pm 14 \mathrm{~nm})$ was evident after $10 \mathrm{~h}$.

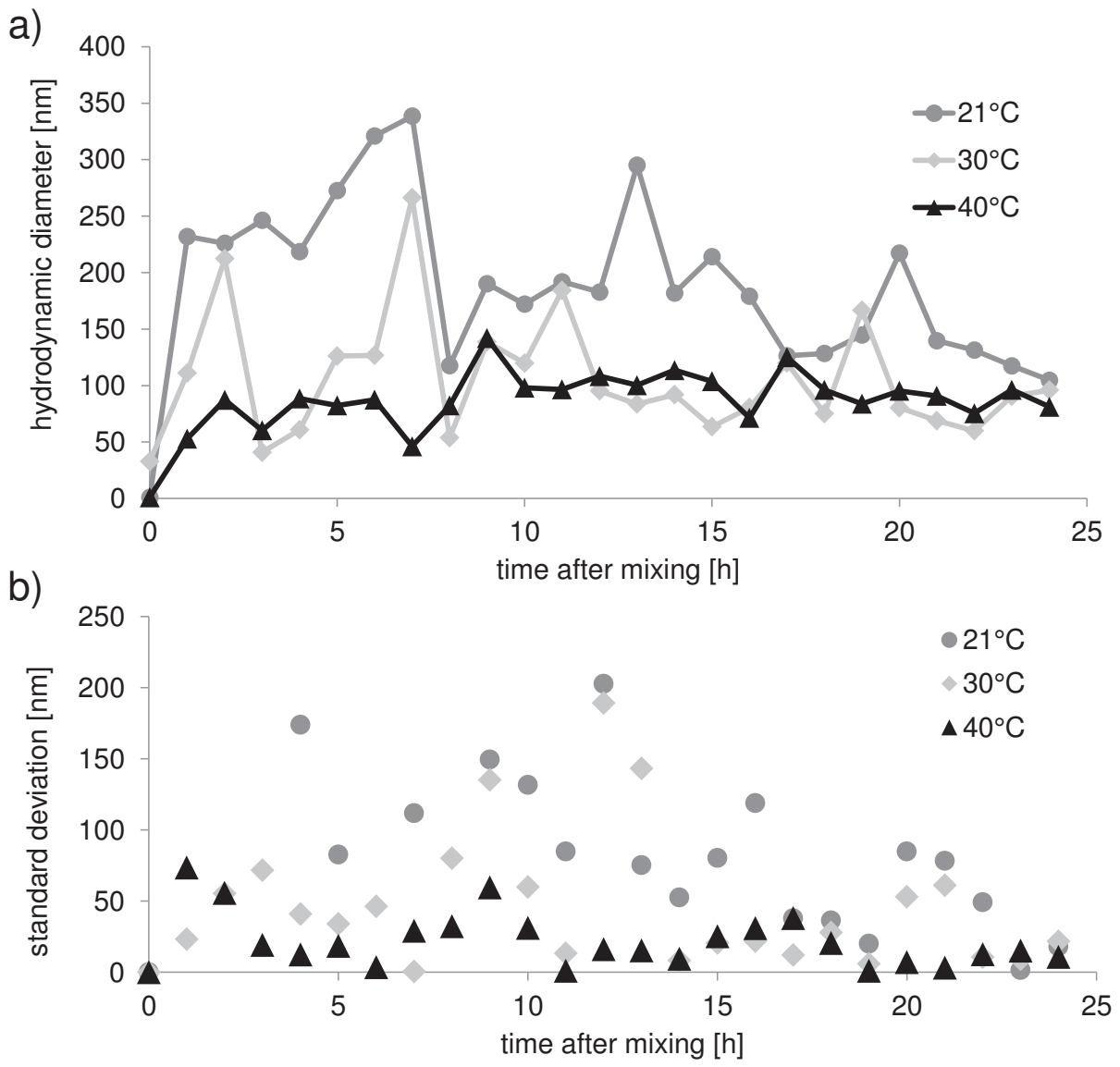

Figure 3.3: a) Hydrodynamic diameters of SNPS, consisting of $25 \% \mathrm{~Np}$ from $\mathrm{Np}_{8}$-PAMAM measured by time-dependent DLS as a function of temperature (data averaged over four measurements) and b) corresponding standard deviations. 
The temperature-dependent particle formation is ascribed to the dynamic character of the supramolecular host-guest binding. After initial rapid ternary complex formation, disassembly and reassembly of the four building blocks is required for well-defined particle formation. At elevated temperatures this rearrangement is enhanced which leads to faster SNP formation. Such a time-dependent particle formation has so far not been reported for SNPs based on CD-Ad host-guest interactions. In all cases reported, SNP formation was observed directly after mixing the supramolecular components. We attribute the difference in dynamics to the stronger and more slowly exchanging complexes of $\mathrm{CB}$ vs. $\mathrm{CD}$.

An important advantage of the use of supramolecular interactions for the formation of SNPs is their responsive character. The triggered disassembly of the SNPs reported here has been induced by the reductant $\mathrm{Na}_{2} \mathrm{~S}_{2} \mathrm{O}_{4}$ (Figure 3.4). After addition of the reducing agent, initially observed NPs could not be detected anymore by DLS and SEM. Apparently, the reduction of the MV moieties led to loss of affinity for the $\mathrm{CB}[8]$ cavity and concomitantly to dissociation of the SNPS.

a)

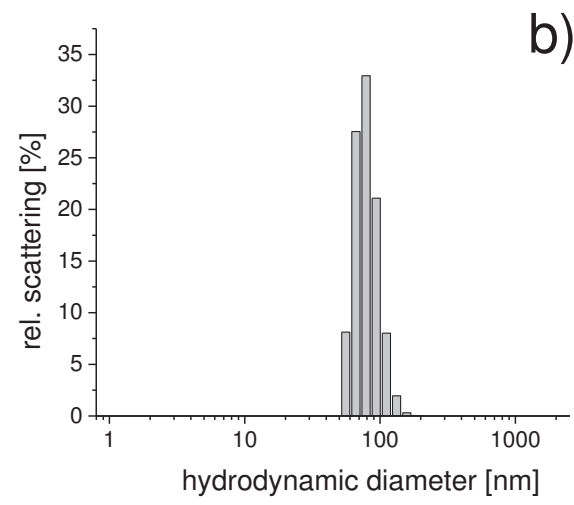

b)

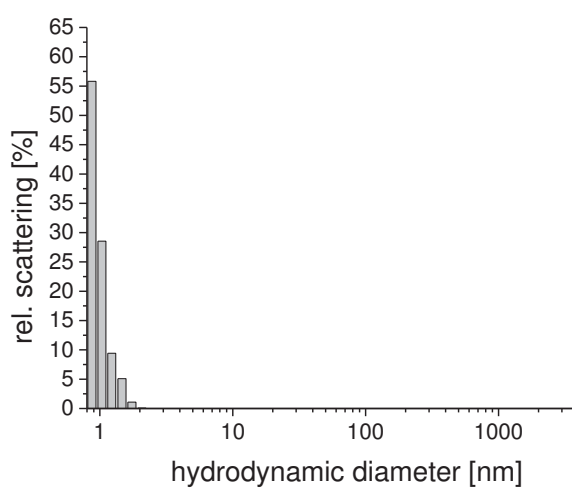

Figure 3.4: DLS analysis of SNP sample prepared with $25 \% \mathrm{~Np}$ from $\mathrm{Np}_{8^{-}}$PAMAM a) after assembly and b) after subsequent addition of $\mathrm{Na}_{2} \mathrm{~S}_{2} \mathrm{O}_{4}$.

\subsubsection{Size tunability of supramolecular nanoparticles}

SNP size control was achieved by varying the ratio of monovalent Np-PEG to multivalent $\mathrm{Np}_{8}$-PAMAM, while keeping the overall $\mathrm{Np}$ concentration constant and maintaining an equimolar $\mathrm{CB}[8]$ :MV:Np stoichiometry. SNPs were observed for all samples as shown by SEM and DLS (Figure 3.4a-g) and the size of the observed SNPs strongly depend on the origin of the Np derivative. In particular, by increasing the amount of Np derived from $\mathrm{Np}_{8}$-PAMAM from $10 \%$ to $35 \%$, an increase in particle size from $57 \pm 11 \mathrm{~nm}$ to $115 \pm 13$ $\mathrm{nm}$ and from $51 \pm 13 \mathrm{~nm}$ to $137 \pm 16 \mathrm{~nm}$ was observed by SEM and DLS, respectively. As shown in Figure 3.4h, the observed SNP size exhibits an apparent linear relationship to the relative amount of $\mathrm{Np}$ derived from $\mathrm{Np}_{8}$-PAMAM. For samples containing $50 \%$ or more of $\mathrm{Np}$ derived from $\mathrm{Np}_{8}$-PAMAM, DLS showed a hydrodynamic diameter beyond $1000 \mathrm{~nm}$, as well as precipitation. 
In general, the formation of SNPs is established by multivalent interactions in the core and monovalent interactions of Np-PEG at the outer surface of the particles. Thereby an equilibrium between capping and crosslinking of Np building blocks is established in time, as indicated by the time-dependent measurements. Stable particle formation requires termination of the multivalent interactions that are established between $\mathrm{Np}_{8}$-PAMAM and MV-PEI in the presence of $\mathrm{CB}[8]$. The results obtained for the size dependence show that more than $50 \%$ monovalent Np-PEG is required to stabilize the NPs. Distinct SNP size control is achieved by fine-tuning the balance of multivalent vs. monovalent interactions. As expected, increasing the concentration of the core forming multivalent dendrimer leads to the formation of larger SNPs.
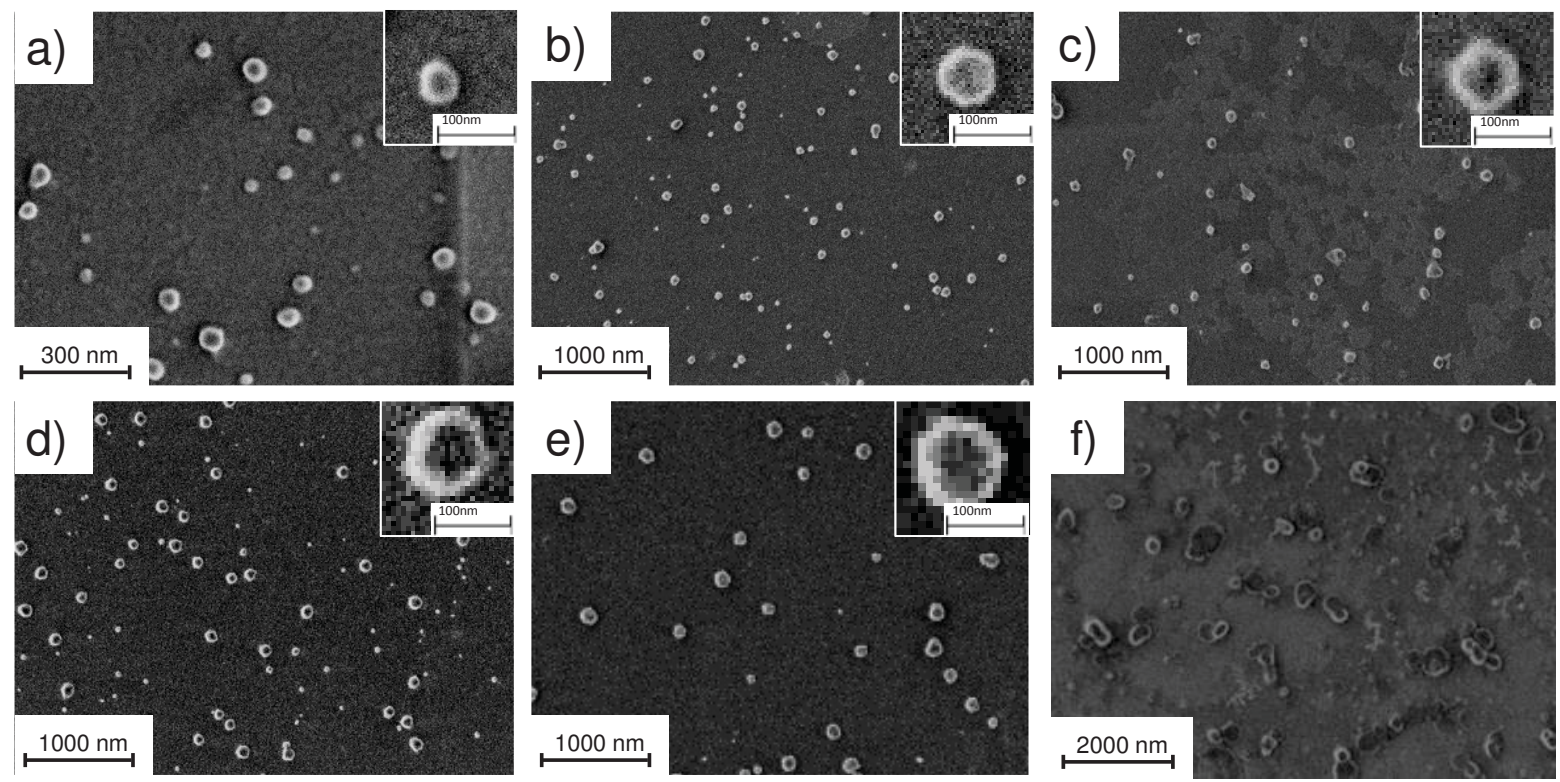

g)

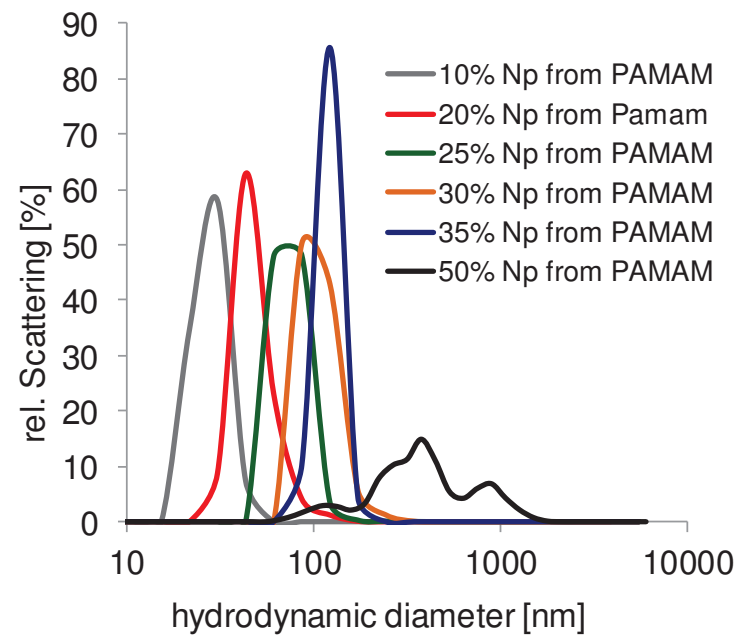

h) 160

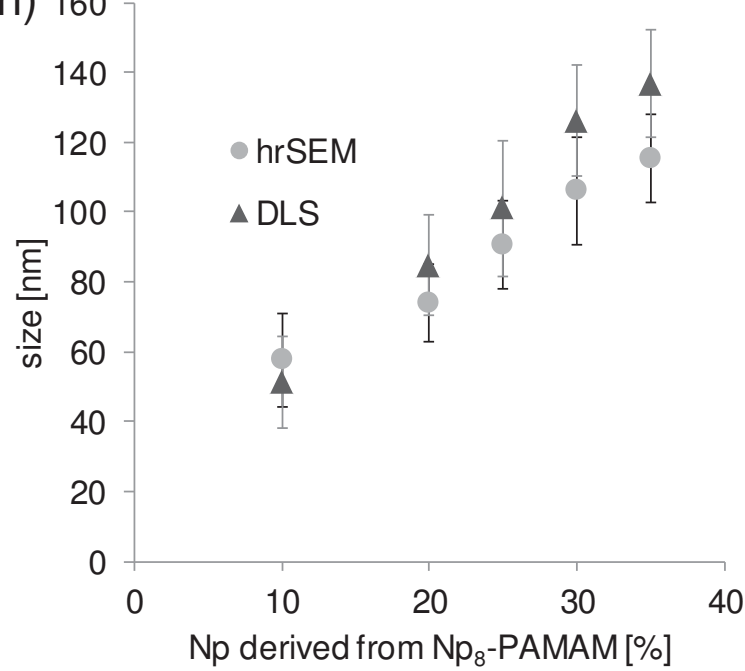

Figure 3.4: Size determination of SNPS prepared with different formulations: SEM images (a-f) of the resulting SNPS, as a function of the Np content derived from No $p_{8}$-PAMAM dendrimer (a: $10 \%$, $b$ : $20 \%$, c: $25 \%$, d: $30 \%$, e: $35 \%$, f: $50 \%$ ) used during supramolecular assembly (using CB[8]:MV:Np $=1: 1: 1$, with $N p$ coming from $N p_{8}-P A M A M$ and $\left.N p-P E G\right)$. g) DLS curves for the different SNP

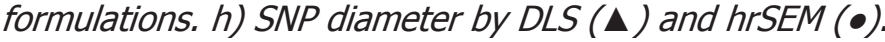




\subsection{Conclusions}

In conclusion, we have developed a novel, versatile strategy for the preparation of SNPs, in which $\mathrm{CB}[8]$ acts as an intermolecular connector between MV-PEI, Np 8 -PAMAM and NpPEG upon formation of a ternary supramolecular CT-complex. Of particular importance are the observed assembly kinetics and the size-focusing in time which indicates progression towards thermodynamic equilibrium. The distinct size tunability as well as the stimulus-responsive particle disassembly make such NPs a potential candidate for biomedical application in drug delivery, peptide therapeutics as well as in vivo sensing.

\subsection{Acknowledgements}

Mark Smithers is acknowledged for the SEM images.

\subsection{Experimental Section}

\subsubsection{Materials}

Starting materials for organic synthesis were obtained from Sigma-Aldrich and used as received. Highly branched poly(ethylene imine) (PEI, $M_{W}=10 \mathrm{kDa}$ ) was purchased from Polysciences Inc. and contained primary, secondary, and tertiary amine groups in a ratio of 25/50/25. 1-(1-Undecyl-11-thiol)-1-methyl-4,4-bipyridinium and 2-naphthoxyterminated poly(ethylene glycol) monomethyl ether were prepared according to literature procedures. $^{24,25}$ Cucurbit[8]uril (CB[8]) was purchased from Strem Chemicals and its purity was assessed by microcalorimetric titration against paraquat. Deuterated solvents used for NMR spectroscopy were purchased from Cambridge Isotope and the water used

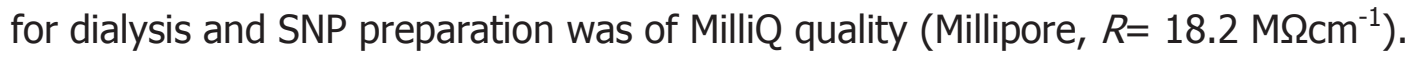

\subsubsection{Synthetic procedures}

\section{Perfluorophenyl 6-(naphthalen-2-yloxy)hexanoate}

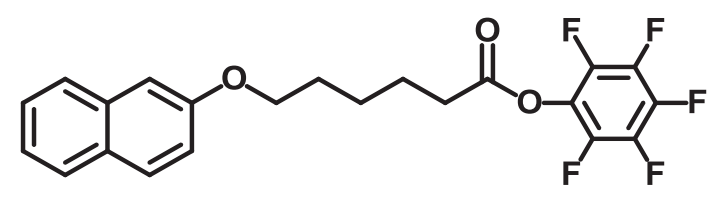

Carboxylic acid activation of 2-naphthol-1hexanoic acid was carried out by dissolving 2naphthol-1-hexanoic acid (0.5 g, $2.05 \mathrm{mmol})$ in $230 \mathrm{~mL}$ THF. Afterwards pentafluorophenol $(0.509 \mathrm{~g}, 2.77 \mathrm{mmol})$ and DCC $(0.570 \mathrm{~g}, 2.77 \mathrm{mmol})$ were added and the solution subsequently stirred for 2 days at RT. THF was removed in vacuum and the residue dissolved in $\mathrm{CH}_{2} \mathrm{Cl}_{2}$ and washed three times with saturated $\mathrm{NaHCO}_{3}$ solution. The organic layer was dried with $\mathrm{MgSO}_{4}$ and the solvent evaporated before the 2-naphthol-1-hexanoic acid pentafluorophenol ester was purified by flash column chromatography over silica gel with $\mathrm{CH}_{2} \mathrm{Cl}_{2}$ as eluent $(\mathrm{RF}=0.83)$. Yield: $0.198 \mathrm{~g}(25 \%)$. ${ }^{1} \mathrm{H}-\mathrm{NMR}\left(400 \mathrm{MHz}_{1} \mathrm{CDCl}_{3}\right): \delta$ 
(ppm) 7.78-7.70 (m, 3H), 7.46-7.29 (m, 2H), 7.21-7.13 (m, 2H), $4.15(\mathrm{t}, 2 \mathrm{H}), 2.80(\mathrm{t}$, $2 \mathrm{H}), 2.01(\mathrm{~m}, 4 \mathrm{H})$. Mass: (ESI) calculated: $410.1(\mathrm{M})$, found: $411.3(\mathrm{M}+\mathrm{H})^{+}$.

Octa-(E)-5-naphthol-pentanoyl-poly(amidoamine)-dendrimer $\quad$ (Generation $\quad 1) \quad\left(\mathrm{Np}_{8}=\right.$ PAMAM)

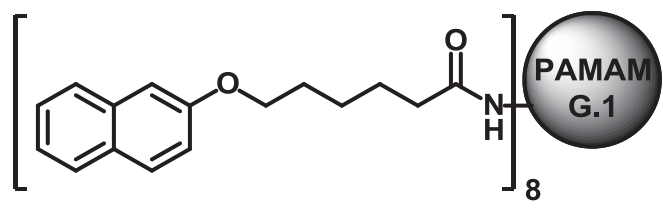

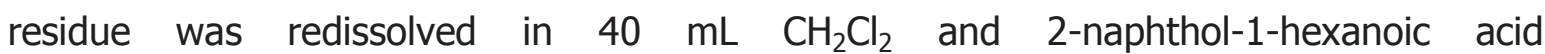
pentafluorophenol ester $(0.074 \mathrm{~g}, 0.178 \mathrm{mmol})$ was added. The solution was stirred for $24 \mathrm{~h}$ at RT under nitrogen atmosphere. The solvent was evaporated and the product isolated by repeated precipitation with ether and hexane. Yield: $20 \mathrm{mg}(35.3 \%) .{ }^{1} \mathrm{H}-\mathrm{NMR}$ (400 MHz, DMSO): $\delta(p p m)$ 7.9-7.75 (m, 44H), 7.5-7.35 (m, 8), 7.35-7.25 (m, 16H), 7.2$7.1(\mathrm{~m}, 8) 4.05(\mathrm{t}, 16 \mathrm{H}), 3.20-3.00(\mathrm{~m}, 40 \mathrm{H}), 2.7-2.60(\mathrm{~m}, 24 \mathrm{H}), 2.45-2.35(\mathrm{~m}, 12 \mathrm{H})$, 2.25- $2.05(\mathrm{~m}, 38 \mathrm{H}), 1.8-1.65(\mathrm{~m}, 32 \mathrm{H})$. Mass: (ESI) calculated: $3239.8(\mathrm{M})$, found: $3241.2(\mathrm{M}+\mathrm{H})^{+}$and calculated: $1620.9(\mathrm{M}+2)^{2+}$ found: $1621.4(\mathrm{M}+2 \mathrm{H})^{2+}$.

N-Poly(ethylene imine)-7-(3-(1-(1-undecyl-11-thiol)-1-methyl-4,4-bipyridinium thio)-2,5dioxo pyrrolidin-1-yl)heptanamide (MV-PEI)

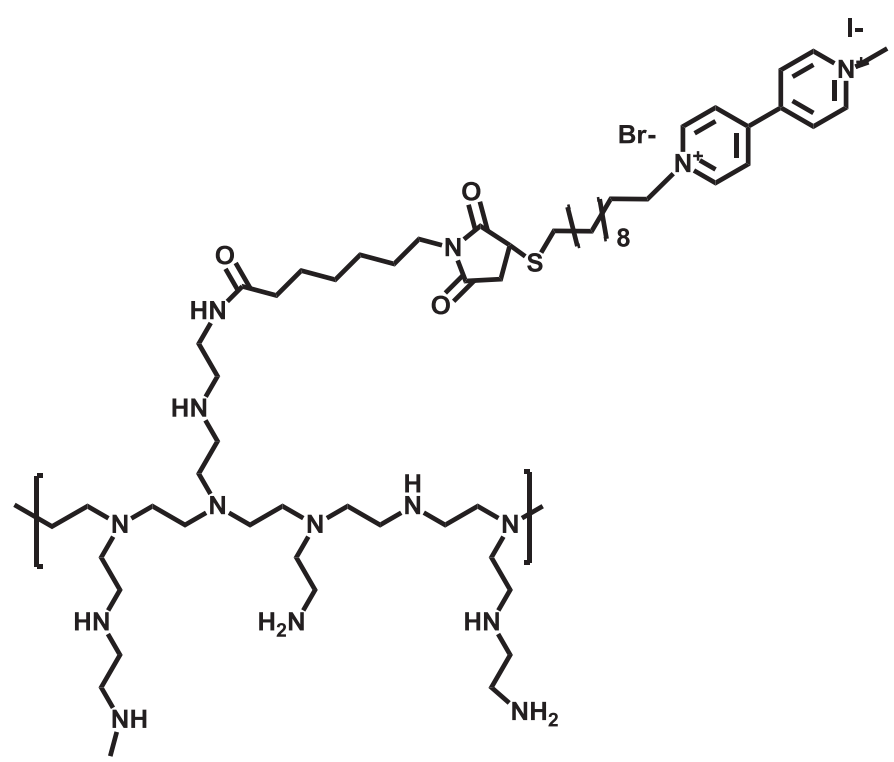

Poly(ethylene imine) $\left[\mathrm{M}_{\mathrm{W}} \sim 10000\right.$ $\mathrm{g} / \mathrm{mol}$ ] (132 mg, $13.2 \mu \mathrm{mol}$ ) was dissolved in $35 \mathrm{~mL}$ dry DMSO and a solution of maleimidohexanoic acid $N$-hydroxy succinimide ester (61 $\mathrm{mg}, 197.86 \mu \mathrm{mol}, 15$ equiv) in 3 $\mathrm{mL}$ DMSO added under inert conditions. The obtained solution was stirred for 1 day at $50{ }^{\circ} \mathrm{C}$. The second reaction step was carried out after cooling down the reaction mixture to RT. Phosphate buffer $(30 \mathrm{~mL}$ ) was added and the $\mathrm{pH}$ of the reaction mixture decreased to

6.9 (controlled via pH-meter). 1-(1-Undecyl-11-thiol)-1-methyl-4,4-bipyridinium (110 mg, $199.98 \mu \mathrm{mol}, 15.16$ equiv) was dissolved and added to the reaction mixture. The solution was stirred overnight at RT and purification was carried out by dialysis (molecular cut-off 
$10.000 \mathrm{~g} / \mathrm{mol}$ ). The product was lyophilized and analyzed by ${ }^{1} \mathrm{H}-\mathrm{NMR}$ and UV/Vis spectroscopy.

As is visible in Figure 3.5, the UV absorption spectrum of the MV- grafted polymer shows the characteristic UV absorbance band of 1-(1-undecyl-11-thiol)-1-methyl-4,4bipyridinium at $268 \mathrm{~nm}$. To determine how many MV were grafted onto the polymer, a calibration curve of 1-(1-undecyl-11-thiol)-1-methyl-4,4-bipyridinium in water was prepared. Thereby, on average $4 \mathrm{MV}$ moieties were found to be grafted to a polymer chain.

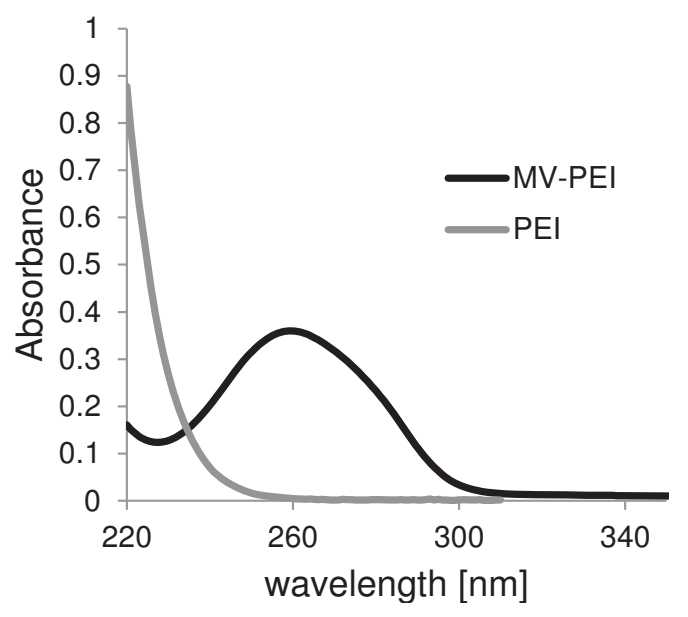

Figure 3.5: UV absorbance of MV-PEI and PEI in aqueous solution.

\subsubsection{Methods}

Supramolecular nanoparticle assembly

For the preparation of size-tunable supramolecular NPs utilizing $\mathrm{CB}[8]$ as supramolecular host, various aqueous solutions of Np-PEG and Np-PAMAM in DMSO were prepared before mixing. Concentration of MV-PEI and $\mathrm{CB}[8]$ were kept the same regardless which size of NPs were formed. E.g. for preparing a solution having 20\% Np entities derived from the aliphatic dendrimer, $1000 \mu \mathrm{L}$ MV-PEI $(0.336 \mu \mathrm{M})$ was added to a previously

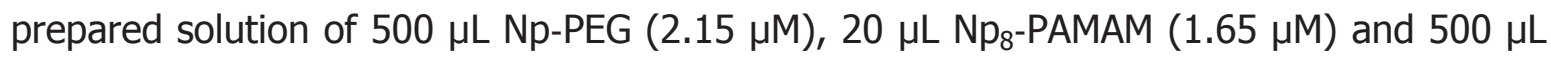
$\mathrm{CB}[8](2.688 \mu \mathrm{M})$. The sample was mixed, combined and kept at RT for 2 days before DLS and SEM analysis.

\section{SNP formation in time}

The kinetics of the formation of supramolecular NPs was analyzed by carrying out continuous DLS measurements over $24 \mathrm{~h}$ at different temperatures. Therefore the required building block solutions were mixed as previously described and the SNP size analysis started directly after mixing. The samples measured at $30^{\circ} \mathrm{C}$ and $40^{\circ} \mathrm{C}$ were warmed up utilizing an oil bath. DLS data were observed directly after mixing, which 
implies, that stabilization of the NP temperature was not yet reached for the starting measurements. In contrast to regular DLS measurements, single measurements were done for each reported data point. For samples prepared at $21^{\circ} \mathrm{C}$ and $30^{\circ} \mathrm{C}$, distinct particle formation was confirmed by measuring the size of the NP sample $48 \mathrm{~h}$ after sample preparation.

\section{Reductive SNP disassembly}

SNP disassembly was carried out by adding $10 \mu \mathrm{L}$ aqueous $\mathrm{Na}_{2} \mathrm{~S}_{2} \mathrm{O}_{4}(5 \mathrm{mmol})$ to the SNP sample under inert conditions. Particle size analysis was carried out via DLS at the following day.

\subsubsection{Equipment}

\section{Dynamic light scattering (DLS)}

DLS experiments were performed with a Zetatrac and Nanotrac by Anaspec operating with a Microtrac FLEX Operating Software at $25^{\circ} \mathrm{C}$ using a laser wavelength of $780 \mathrm{~nm}$ at a scattering angle of $90^{\circ}$. The observed sizes and standard derivations of the supramolecular NPs were calculated by taking an average of 5 measurements. In total, particles with sizes ranging from 0.8 to $6400 \mathrm{~nm}$ were detected. The DLS curves shown in this chapter are representative for the individual measurements.

\section{Scanning electron microscopy (SEM)}

All SEM images were taken with a Carl-Zeiss 1550 Gemini field emission scanning electron microscope. Therefore the aqueous SNP solutions were drop-cast on a Formvar coated copper TEM grid. The solution was dried and analyzed without further treatment of the samples.

\section{$\underline{\text { UV/Vis spectroscopy }}$}

UV/Vis absorption spectra were recorded using a Perkin Elmer Lambda 850 UV-Vis spectrometer. To investigate the formation of a charge transfer band while SNP selfassembly, SNPs were prepared as described previously. The formation of particles was controlled using DLS. For UV/Vis analysis, the sample was concentrated 13.5 times to observe sufficient UV absorbance. As control experiments, CB[8] and a SNP formulation in absence of $\mathrm{CB}[8]$ was prepared in the same concentration, and the UV/Vis absorbance determined.

\section{Fluorescence spectroscopy}

Fluorescence spectra were recorded using a Perkin Elmer LS 55 fluorescence equipped with a high energy pulsed Xenon source for excitation. Emission and excitation slits were kept constant at $2.5 \mathrm{~cm}$. To evaluate if SNP assembly is driven by the formation of the heteroternary complex between $\mathrm{CB}[8]: \mathrm{MV}: \mathrm{Np}$, the SNP formulation was prepared in 
presence and absence of $\mathrm{CB}[8]$. And the fluorescence emission of both solution directly controlled after mixing using an excitation wavelength of $266 \mathrm{~nm}$.

\section{NMR spectroscopy}

${ }^{1} \mathrm{H}$ and ${ }^{13} \mathrm{C}$ NMR spectra were recorded on Bruker $400 \mathrm{MHz}$ and $600 \mathrm{MHz}$ spectrometer. ${ }^{1} \mathrm{H}$ and ${ }^{13} \mathrm{C}$ chemical shift values, $400 \mathrm{MHz}$ and $150 \mathrm{MHz}$, are reported as $\delta$ using the residual solvent signal as internal standard.

\section{Mass spectrometry}

Mass analysis was performed using the matrix-assisted laser desorption ionization (MALDI) and electrospray ionization using a Voyager DE-RP and a micromass LCT from Waters/Micromass, respectively.

\subsection{References}

1. J.-M. Lehn, Proceedings of the National Academy of Sciences, 2002, 99, 4763-4768.

2. X. Y. Ling, I. Y. Phang, H. Schönherr, D. N. Reinhoudt, G. J. Vancso and J. Huskens, Small, 2009, 5, 1428-1435.

3. T. Aida, E. W. Meijer and S. I. Stupp, Science, 2012, 335, 813-817.

4. M. Auffan, J. Rose, J.-Y. Bottero, G. V. Lowry, J.-P. Jolivet and M. R. Wiesner, Nature Nanotechnology, 2009, 4, 634-641.

5. S. K. Sahoo and V. Labhasetwar, Drug Discovery Today, 2003, 8, 1112-1120.

6. J. Panyam and V. Labhasetwar, Advanced Drug Delivery Reviews, 2003, 55, 329-347.

7. T. Doane and C. Burda, Advanced Drug Delivery Reviews, 2013, 65, 607-621.

8. Y. Bae, S. Fukushima, A. Harada and K. Kataoka, Angewandte Chemie International Edition, 2003, 42, 4640-4643.

9. W. Xiao, W.-H. Chen, J. Zhang, C. Li, R.-X. Zhuo and X.-Z. Zhang, Journal of Physical Chemistry B, 2011, 115, 13796-13802.

10. M. E. Davis, J. E. Zuckerman, C. H. J. Choi, D. Seligson, A. Tolcher, C. A. Alabi, Y. Yen, J. D. Heidel and A. Ribas, Nature, 2010, 464, 1067-1070.

11. J. E. Zuckerman, C. H. J. Choi, H. Han and M. E. Davis, Proceedings of the National Academy of Sciences, 2012, 109, 3137-3142.

12. D. W. Bartlett and M. E. Davis, Bioconjugate Chemistry, 2007, 18, 456-468.

13. H. Wang, S. Wang, H. Su, K.-J. Chen, A. L. Armijo, W.-Y. Lin, Y. Wang, J. Sun, K.-i. Kamei, J. Czernin, C. G. Radu and H.-R. Tseng, Angewandte Chemie International Edition, 2009, 48, 4344-4348.

14. C. Fasting, C. A. Schalley, M. Weber, O. Seitz, S. Hecht, B. Koksch, J. Dernedde, C. Graf, E.-W. Knapp and R. Haag, Angewandte Chemie International Edition, 2012, 51, 1047210498.

15. A. Mulder, J. Huskens and D. N. Reinhoudt, Organic \& Biomolecular Chemistry, 2004, 2, 3409-3424.

16. X. Y. Ling, D. N. Reinhoudt and J. Huskens, Pure and Applied Chemistry, 2009, 81, 22252233. 
17. S. Liu, C. Ruspic, P. Mukhopadhyay, S. Chakrabarti, P. Y. Zavalij and L. Isaacs, Journal of the American Chemical Society, 2005, 127, 15959-15967.

18. E. Masson, X. Ling, R. Joseph, L. Kyeremeh-Mensah and X. Lu, RSC Advances, 2012, 2, 1213-1247.

19. H.-J. Kim, J. Heo, W. S. Jeon, E. Lee, J. Kim, S. Sakamoto, K. Yamaguchi and K. Kim, Angewandte Chemie International Edition, 2001, 40, 1526-1529.

20. E. A. Appel, J. Dyson, J. del Barrio, Z. Walsh and O. A. Scherman, Angewandte Chemie International Edition, 2012, 51, 4185-4189.

21. E. A. Appel, J. d. Barrio, J. Dyson, L. Isaacs and O. A. Scherman, Chemical Science, 2012, 3, 2278-2281.

22. J. Zhang, R. J. Coulston, S. T. Jones, J. Geng, O. A. Scherman and C. Abell, Science, 2012, 335, 690-694.

23. A. González-Campo, M. Brasch, D. A. Uhlenheuer, A. Gómez-Casado, L. Yang, L. Brunsveld, J. Huskens and P. Jonkheijm, Langmuir, 2012, 28, 16364-16371.

24. H. Kotani, K. Ohkubo, Y. Takai and S. Fukuzumi, Journal of Physical Chemistry B, 2006, 110, 24047-24053.

25. O. A. Scherman, Polymer Preprints, 2010, 51 (1), 299-300. 


\section{Chapter 4}

\section{Effects of Molecular Weight and Valency of Guest- modified Poly(ethylene glycol)s on the Stability, Size and Dynamics of Supramolecular Nanoparticles}

The influence of polymer length and the valency of the guest-modified poly(ethylene glycol) (PEG) on the appearance, stability, size tunability and formation dynamics of supramolecular nanoparticles (SNPs) has been studied in this chapter. SNPs were formed by molecular recognition of supramolecular building blocks with host or guest moieties, providing ternary complexes of cucurbit[8]uril, methyl viologen and naphthol (Np). SNP assembly was carried out using monovalent Np-modified oligo(ethylene glycol)s and PEGs with molecular weights ranging between 133 and $20000 \mathrm{~g} / \mathrm{mol}$. SNP formation and stoichiometry controlled size tuning was observed for SNPs prepared with Np-modified PEGs of polymer lengths between 900 and $20000 \mathrm{~g} / \mathrm{mol}$, whereas no distinct assemblies were formed using the shorter Np-functionalized tri(ethylene glycol). Use of the divalent Np-functionalized PEG $(5000 \mathrm{~g} / \mathrm{mol}$ ) slowed down the SNP assembly dynamics and prohibited the formation of distinct SNPs at RT within the first week after mixing of the components. In contrast, SNPs with distinct sizes were obtained after warming up the building blocks to $40^{\circ} \mathrm{C}$ for $12 \mathrm{~h}$. 


\subsection{Introduction}

Nanomaterials are synthetic materials with dimensions smaller than a few hundreds of nanometers and hold great promise in catalysis, ${ }^{1}$ as analytical sensors, ${ }^{2,3}$ and for electronic ${ }^{4}$ or biomedical applications. ${ }^{5,6}$ Nanoparticles (NPs) in particular are often reported as multifunctional diagnostic and therapeutic tools to protect and deliver active compounds into targeted tissue in vivo, while preventing their degradation and minimizing undesired side-effects. ${ }^{7-9}$

NPs are generally not colloidally stable in biological fluids as the high surface energy of the NPs and the high ionic strength of the fluids lead to uncontrolled particle aggregation. ${ }^{10}$ Furthermore, physiological environments such as blood, interstitial fluids or cellular cytoplasma, contain mixtures of proteins which adsorb to the NP surface by non-specific interactions. ${ }^{11,12}$ These adsorbed proteins alter the size and interfacial composition of a nanomaterial and often act as opsonins, markers that enhance phagocytosis by the mononuclear phagocyte system (MPS). ${ }^{13,14}$ Therefore, polymerbased steric stabilization layers are required to prevent uncontrolled protein adsorption and rapid clearance of NPs from the bloodstream to increase the pharmacokinetics and biodistribution of NPs in vivo. ${ }^{15}$

Surface modification with oligo(ethylene glycol) or poly(ethylene glycol) (PEG) called "PEGylation" has been the most widely used method to create a steric barrier around NPs to prevent nonspecific adsorption from complex media. ${ }^{16}$ Using this strategy, a variety of NP systems has been reported in which the in vivo circulation time of PEGylated NPs was extended compared to the uncoated parent particles. ${ }^{17,18}$ Apparently, the PEG length ${ }^{19,20}$ and density ${ }^{21}$ have strong, inhibiting effects on the serum protein adsorption and macrophage uptake and therefore result in increased blood circulation times.

Supramolecular nanoparticles (SNPs) are formed by multiple monovalent and multivalent building blocks brought together by specific non-covalent interactions. ${ }^{22}$ The chemical versatility of the SNP assembly supports the inclusion of active compounds into the particles, and has been used for applications such as the delivery of RNA/DNA, ${ }^{23-25}$ drugs $^{26}$ imaging agents ${ }^{27}$ or transcription factors. ${ }^{28}$ Assembled by multivalent electrostatic or host-guest interactions in the core, SNP formation strategies require additional stabilization by hydrophilic polymers assembling in the SNP shell. This colloidal SNP stabilization can be achieved, for example, by host-guest interactions of cyclodextrin and adamantane using a monovalent adamantyl-functionalized PEG interacting with the multivalent CD-functionalized polymer thus constituting the SNP shell. $23,24,26,29,30$ The shielding enabled by the PEG shell provided the stability of polycation-nucleic acid composites in biological fluids, and the long-term circulation of the SNPs in vivo. ${ }^{31}$ 
In Chapter 3, a novel SNP formation strategy was presented, in which the SNP assembly is promoted by multiple charge transfer complexes between cucurbit[8]uril (CB[8]), methyl viologen (MV) and naphthol (Np). The relatively high binding affinity of this ternary complex led to SNPs, the assembly dynamics of which appeared to be markedly slower than that observed for cyclodextrin assemblies. Nonetheless, the interplay between multivalent and monovalent Np-bearing guest molecules provided size tuning of the SNPs similar to the SNP formation strategy reported by Tseng and coworkers for the interaction of cyclodextrin and adamantyl-functionalized building blocks. ${ }^{30}$ In the CB[8] and CD strategies, both the colloidal stability as well as the size tuning of the SNPs can be attributed to the shielding effect of the monovalent guest-modified PEG stopper. However, it is unclear to which extent the polymer length and the valency of the stopper affect the SNP shielding.

The aim of the work presented in this chapter is to correlate the structure of the guestmodified PEG stopper with the colloidal stability, size tuning and assembly dynamics of SNPs stabilized by CB[8]/MV/Np interactions. In particular, the effects of the length of the PEG chain and the number (valency) of guest moieties attached to the PEG end on the SNP stability are evaluated in detail.

\subsection{Results and Discussion}

\subsubsection{SNP formation using different shell components}

As schematically shown in Scheme 4.1, SNPs are formed by the self-assembly of MVpoly(ethylene imine) (MV-PEI), Np-poly(amido amine) dendrimer ( $\mathrm{Np}_{8}$-PAMAM), CB[8] and different Np-functionalized PEGs. In the presence of $\mathrm{CB}[8]$, the SNPs assemble by multiple interactions of MV-PEI and $\mathrm{Np}_{8}$-PAMAM in the particle core and of Np-PEG in the SNP shell. Using monovalent Np-conjugated PEG with different lengths, the effect of the polymer length on the formation of SNPs is evaluated. Furthermore, the effect of the valency of the PEG stopper is tested by using a divalent Np-conjugated PEG with a polymer length comparable to that of the monovalent derivative described in Chapter 3. 
a)
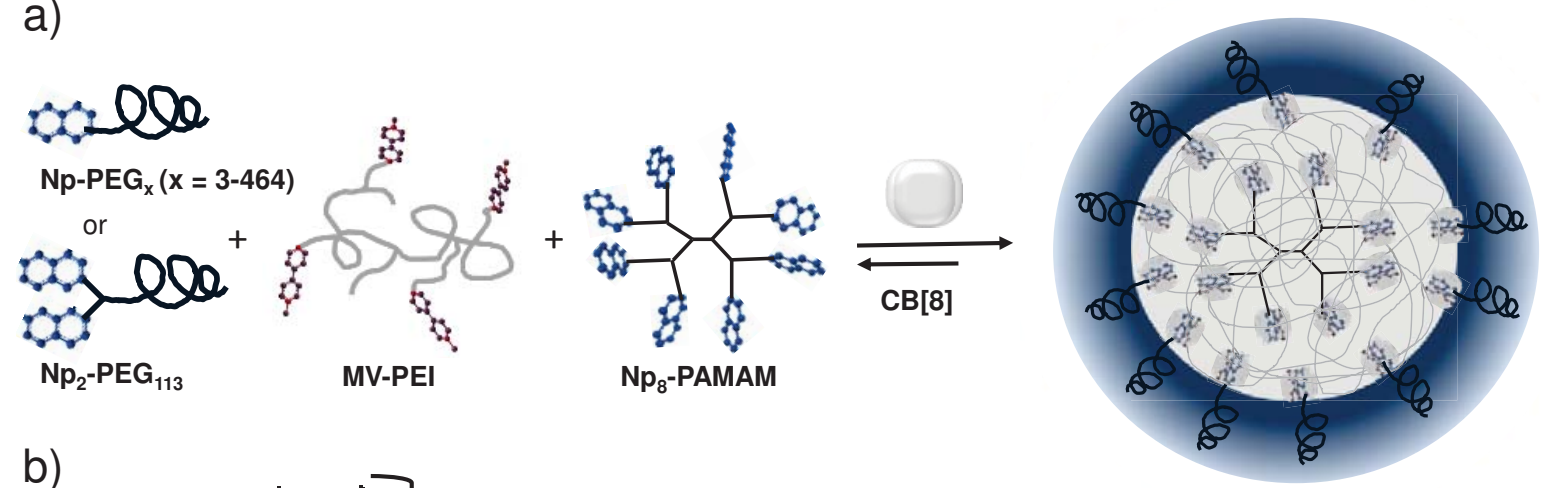

b)

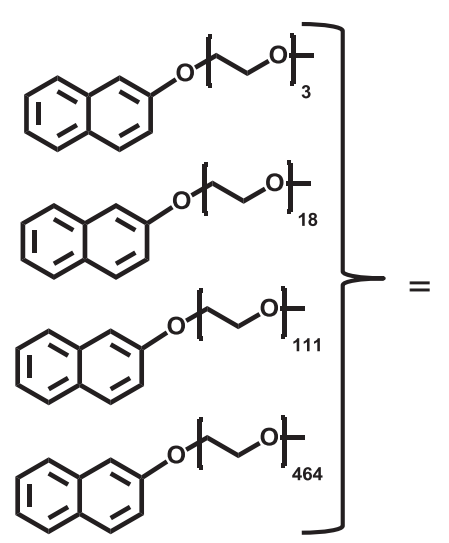

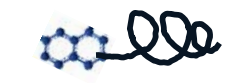

Np-PEG $x(x=3-454)$

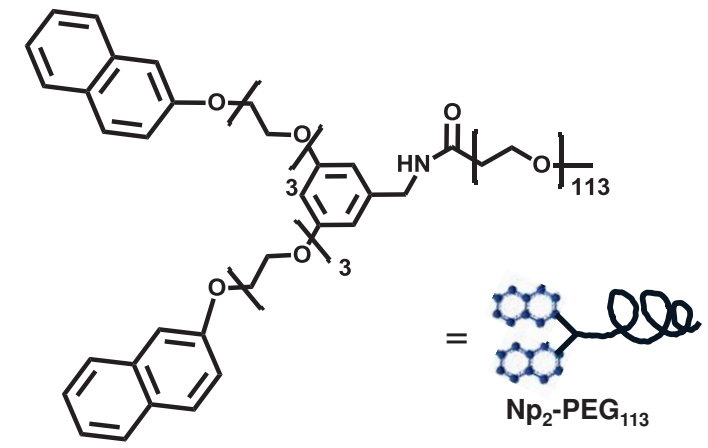

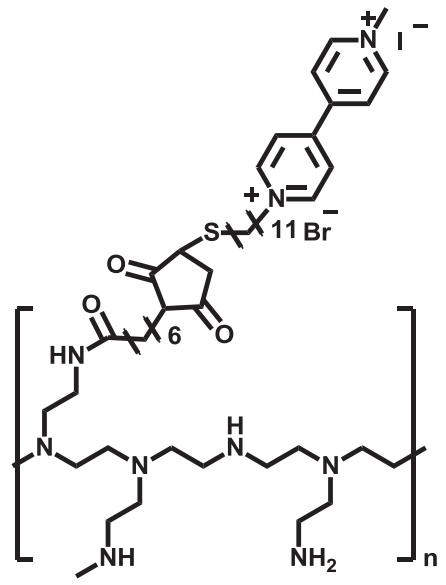

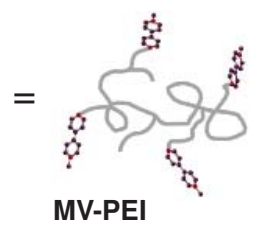<smiles>CC(C)(C)C(C)(C)C(C)(C)C(C)(C)C</smiles>

$\mathrm{Np}_{8}$-PAMAM

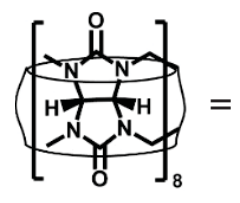

$\mathrm{CB}[8]$

Scheme 4.1: a) Supramolecular nanoparticle (SNP) formation by heteroternary complex formation between cucurbit(8)uril (CB[8]), methyl viologen (MV) and naphthol (Np) moieties using MVfunctionalized poly(ethylene imine) (MV-PEI), Np-functionalized PAMAM G1 dendrimer ( $\mathrm{Np}_{8^{-}}$ $P A M A M), C B[8]$ and different Np-functionalized poly(ethylene glycol)s (Np-PEGs). b) The supramolecular building blocks used for SNP formation: Np-tri(ethylene glycol) (Np-PEG $\left.G_{3}\right), N p-P E G_{18 \text {, }}$ $N p-P E G_{111}, N p-P E G_{464}, N p_{2}-P E G_{113}, M V-P E I, N p_{8}-P A M A M$ and CB[8].

To study the effect of PEG length on SNP formation, various Np-conjugated PEG stopper molecules were synthesized. Four monovalent Np-conjugated PEG derivatives, with 3 or, on average, 18,111 , or 464 repeat units, and a divalent Np-grafted PEG, $\mathrm{Np}_{2}-\mathrm{PEG}_{113}$, were synthesized. As shown in Scheme 4.2, the short Np-PEG 3 was synthesized by the reaction of 2-naphthol with $\mathrm{NaH}$ followed by Williamson ether synthesis using chloroethoxy-ethoxy-ethanol. Np-PEG 18 was synthesized as reported for Np-PEG $\mathrm{P}_{111}$ in Chapter 3 by a substitution reaction of 2-naphthol with poly(ethylene glycol) methyl ether tosylate. 
The longest monovalent Np-conjugated PEG, Np-PEG 464 was prepared by reaction of 2naphthoylchloride with PEG methyl ether amine in dichloromethane. To prepare $\mathrm{Np}_{2}-$ $\mathrm{PEG}_{113}$ (Scheme 4.2d), first the hydroxyl group of Np-PEG 3 was converted to the more reactive bromide using $\mathrm{PBr}_{3}$ in toluene. This bromide (4) was reacted with 3,5-dihydroxy benzonitrile under reflux using $\mathrm{K}_{2} \mathrm{CO}_{3}$ and 18-crown-6 in acetone to obtain the divalent guest unit $\mathbf{5}$. The nitrile functionality of $\mathbf{5}$ was converted to an amine by the hydrogenation with $\mathrm{H}_{2}$ in the presence of Raney-nickel as the catalyst. Following, $\mathrm{Np}_{2}-$ $\mathrm{PEG}_{113}$ (6) was obtained by reaction with methyl-poly(ethylene glycol) $\mathrm{N}$ hydroxysuccinimide ester in $\mathrm{CH}_{2} \mathrm{Cl}_{2}$.

a)<smiles>Oc1ccc2ccccc2c1</smiles>
2. ${ }^{\mathrm{cl}} \mathrm{O} \sim \mathrm{NO}_{\mathrm{OH}} / \mathrm{THF}$<smiles>OCCOCCOCCOc1ccc2ccccc2c1</smiles>

(1)

b)<smiles>Oc1ccc2ccccc2c1</smiles>

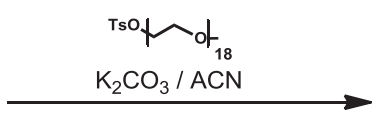<smiles>COCCOc1ccc2ccccc2c1</smiles>

(2)

c)<smiles>O=C(Cl)c1ccc2ccccc2c1</smiles>

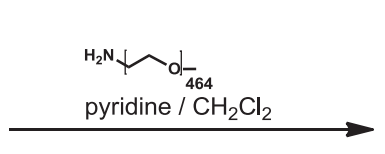<smiles>CC(C)(C)OCCNC(=O)c1ccc2ccccc2c1</smiles>

(3)

d)

(1)

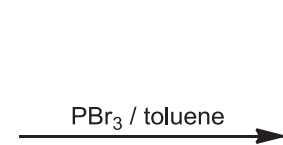

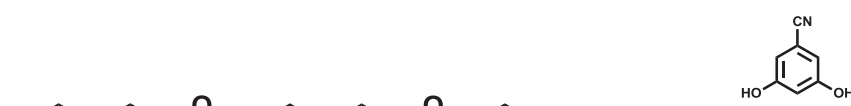

(4)

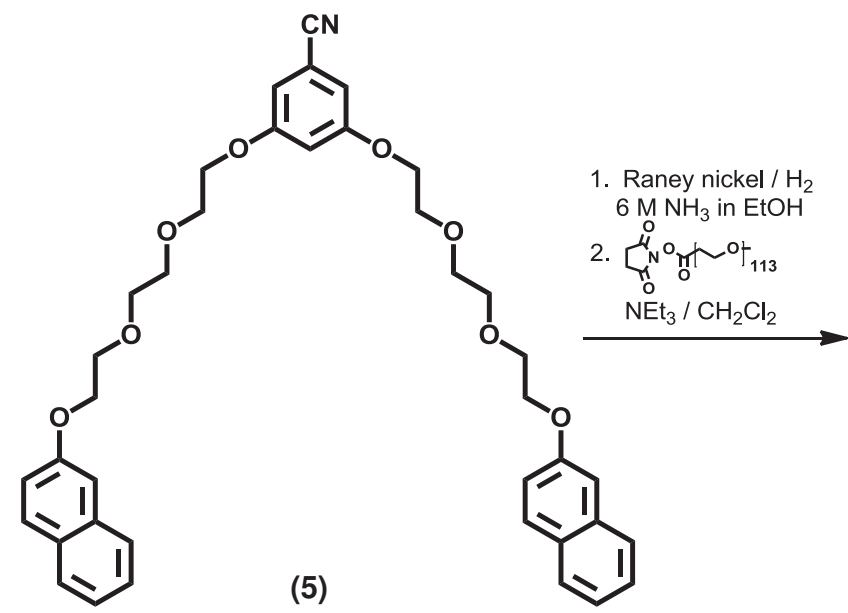<smiles>CCCOCCOc1ccc2ccccc2c1</smiles>

(6)

Scheme 4.2: Synthetic routes towards the monovalent Np-conjugated PEG derivatives (a-c) and the divalent $N p_{2}-P E G_{113}$ used in this study. 


\subsubsection{SNP formation with monovalent Np-PEG of varying length}

The formation of SNPs was studied using a $0.67 \mu \mathrm{M}$ concentration of $\mathrm{CB}[8], \mathrm{MV}$ and $\mathrm{Np}$, while keeping the molecular recognition moieties in a stoichiometric ratio of 1:1:1. Therefore, $\mathrm{Np}_{8}$-PAMAM was dissolved in DMSO and aqueous solutions of MV-PEI, CB[8] and $\mathrm{Np}-\mathrm{PEG}_{\mathrm{x}}(\mathrm{x}=3,18,111,464)$ were prepared prior to mixing. Size tuning of the SNPS was assessed by varying the concentrations of the Np-PEG derivative and the $\mathrm{Np}_{8}$-PAMAM dendrimer, while keeping the overall concentration of the Np moieties constant. As for the SNPs reported in Chapter 3 (prepared with Np-PEG ${ }_{111}$ ), the Np-conjugated $P E G_{x}$ was mixed with $\mathrm{Np}_{8}$-PAMAM and $\mathrm{CB}[8]$ prior to addition of MV-PEI. The formation of SNPS was evaluated by DLS and SEM, either 2 or 7 days after mixing, respectively.

As shown in Figure 4.1, self-assembly experiments carried out with $\mathrm{Np}-\mathrm{PEG}_{3}, \mathrm{~Np}_{8}-\mathrm{PAMAM}$, MV-PEI and $\mathrm{CB}[8]$ did not result in distinct SNP formation. Only few particle-shaped structures were observed, which were embedded in organic material. This observation is in accordance with DLS characterization carried out for this SNP formulation. For all concentrations of $\mathrm{Np}_{8}$-PAMAM used during the self-assembly experiments, only irreproducible aggregation or failed measurements were observed.
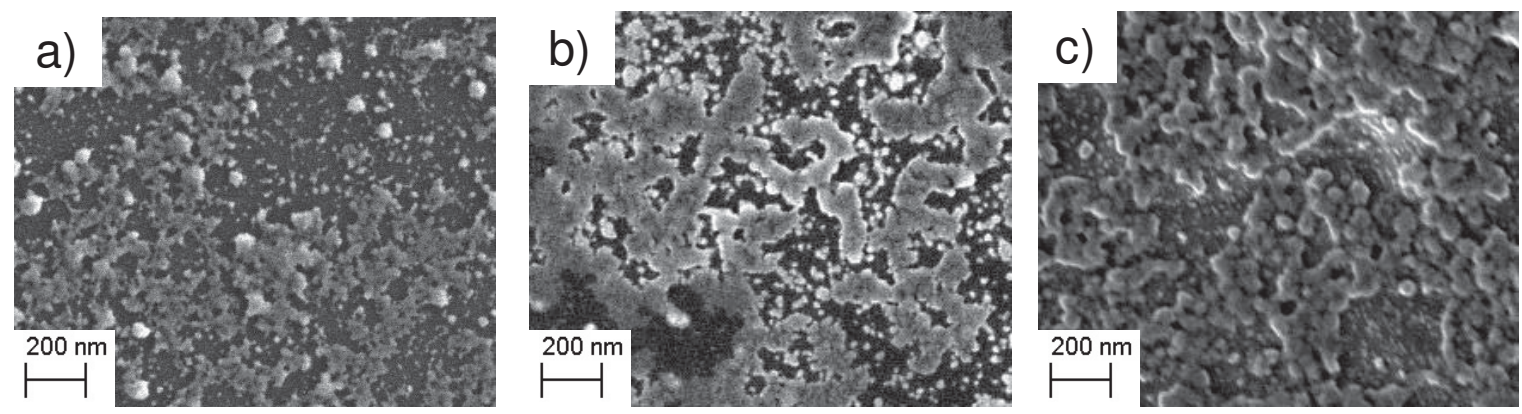

Figure 4.1: SEM images observed for SNP formulations prepared with Np-PEG 3 as a function of Np content derived from $\mathrm{Np}_{8}$-PAMAM dendrimer (a: $10 \%$, b: $20 \%, \mathrm{c}: 30 \%$ ).

In contrast to $\mathrm{Np}-\mathrm{Peg}_{3}$, distinct SNPs were observed using $\mathrm{Np}-\mathrm{PEG}_{18}$ (whilst maintaining $\mathrm{Np}_{8}$-PAMAM, MV-PEI and $\left.\mathrm{CB}[8]\right)$. By increasing the amount of $\mathrm{Np}$ derived from $\mathrm{Np}_{8}-$ PAMAM, while decreasing the amount $\mathrm{Np}$ from $\mathrm{Np}-\mathrm{PEG}_{18}$, the observed SNP size increased from $37 \pm 7 \mathrm{~nm}$ to $65 \pm 13 \mathrm{~nm}$ as measured by SEM and from $50 \pm 3 \mathrm{~nm}$ to 97 $\pm 11 \mathrm{~nm}$ as analyzed by DLS. 

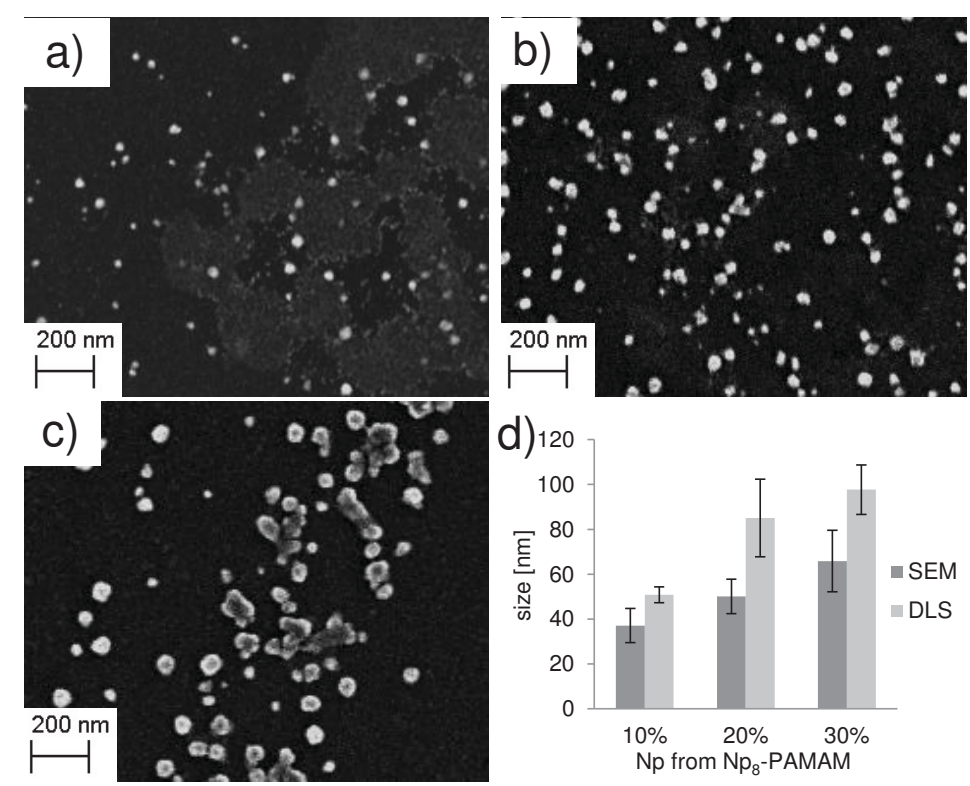

Figure 4.2: Size determination of SNPS prepared with Np-PEG 18: SEM images (a-c) as a function of the $\mathrm{Np}$ content derived from Np $\mathrm{p}_{8}$ PAMAM dendrimer (a: $10 \%, \mathrm{~b}: 20 \%, \mathrm{c}: 30 \%$ ) used during supramolecular assembly while keeping the concentration ratio of CB[8]:MV:Np = 1:1:1. d) SNP diameter as measured by SEM ( $\square$ ) and DLS ( $)$.

As seen in Figure 4.3, distinct SNPs were formed as well for different formulations using $\mathrm{Np}-\mathrm{PEG}_{464}, \mathrm{~Np}_{8}$-PAMAM, MV-PEI and $\mathrm{CB}[8]$. Also in this case, stoichiometric size control was observed by increasing the amount of $\mathrm{Np}$ from $\mathrm{Np}_{8}$-PAMAM from $10 \%$ to $30 \%$, while decreasing the amount of Np-PEG 464 from $90 \%$ to $70 \%$ correspondingly: an increase in SNP size was observed from $35 \pm 4 \mathrm{~nm}$ to $61 \pm 12 \mathrm{~nm}$ by SEM and from $47 \pm 9 \mathrm{~nm}$ to $117 \pm 15 \mathrm{~nm}$ by DLS (Figure 4.3d).
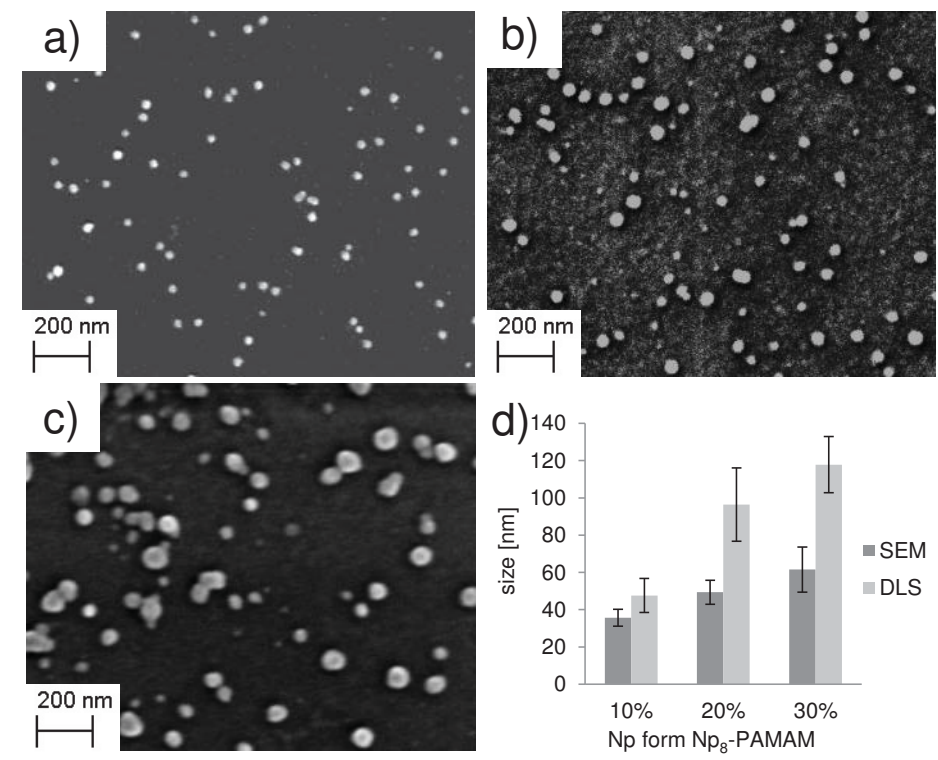

Figure 4.3: Size determinations of SNPs prepared with Np-PEG 464: SEM images (a-c) as a function of the $\mathrm{Np}$ content derived from $N p_{8}-P A M A M$ dendrimer (a: 10\%, b: $20 \%, c: 30 \%$ ) used during supramolecular assembly while keeping the concentration ratio of CB[8]:MV:Np = 1:1:1. d) SNP diameter as measured by SEM ( $\square$ ) and DLS ( $)$. 
In summary, these results illustrate that distinct SNP formation is not possible using Np$\mathrm{PEG}_{3}$ as the stopper assembling in the shell of the SNPs. Most likely the short tri(ethylene glycol) moiety does not provide sufficient stability to the SNPs, because of its marginal steric shielding capacity. Including the results presented in Chapter 3 for Np-PEG 111 , clear SNP formation was observed for SNPs formed using Np-PEG $\mathrm{F}_{18}, \mathrm{~Np}-\mathrm{PEG}_{111}$ and Np$\mathrm{PEG}_{464}$. Remarkably, even the still fairly short Np-PEG derivative grafted with 18 repeat units is apparently capable of providing sufficient colloidal stability to the SNPs. Furthermore, for all SNPs, size tuning was achieved by stoichiometric control of the ratio between the multivalent and monovalent Np components, irrespective of the length of the Np-PEG.

Figure 4.4 shows a comparison of the particle sizes determined by DLS (Figure 4.4a) and SEM (Figure 4.4b). While the SEM diameters show no dependence on PEG length, the hydrodynamic diameters measured by DLS show a slightly upward trend for increasing chain lengths. Also the differences between DLS and SEM sizes increase with increasing percentage of multivalent $\mathrm{Np}$ dendrimer (Figure 4.4c). In all cases, the effect of PEG length on particle size seems to be statistically insignificant.
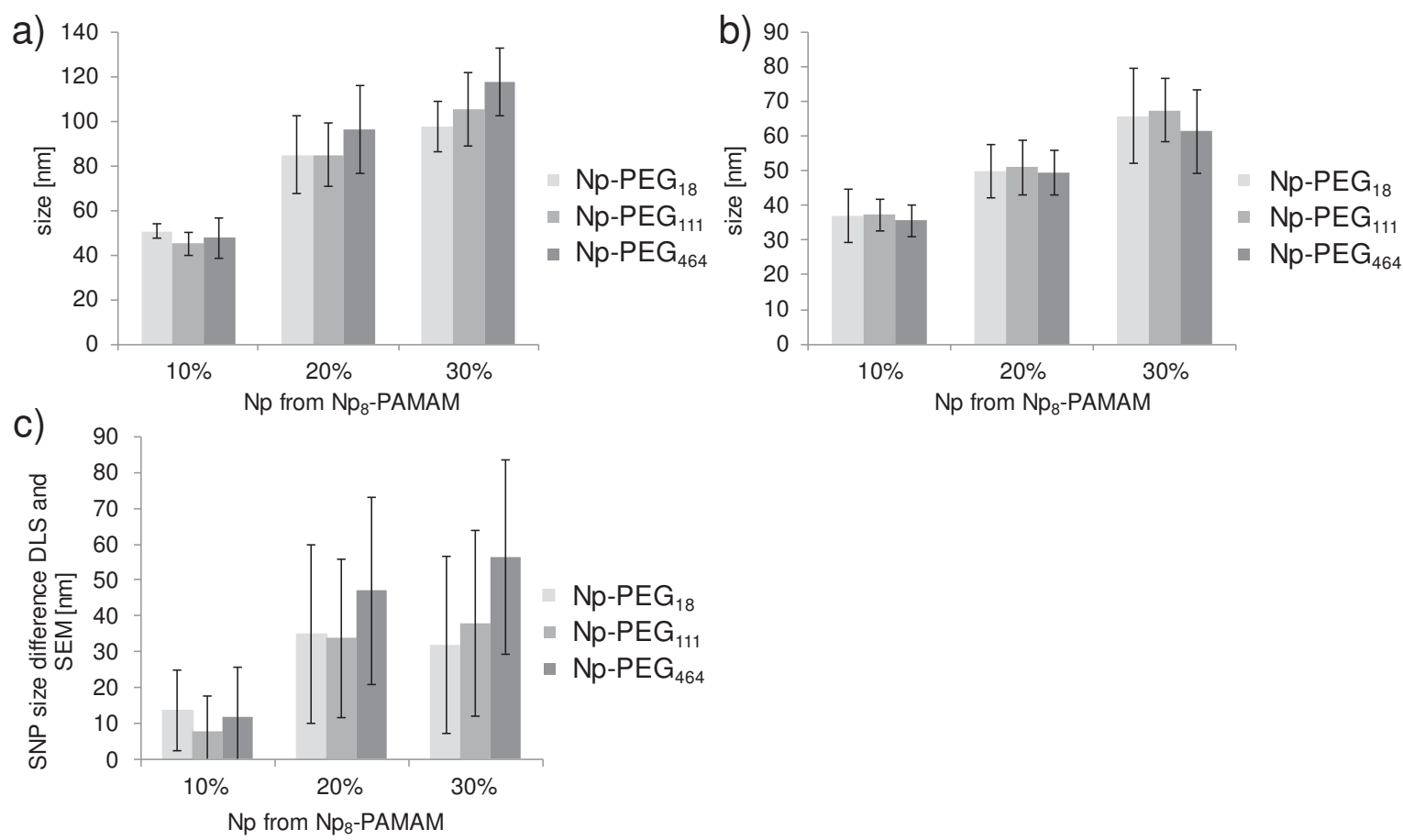

Figure 4.4: Size determination of SNPs prepared with Np-PEG $18(\square), N p-P E G_{111}(\square)$ and Np-PEG 464 ( $)$ : a) by DLS and b) by SEM. c) Difference in hydrodynamic diameter by DLS and SNP diameter by SEM.

It has been reported that the surface area occupied by PEG increases with the length of the polymer. ${ }^{32}$ When assuming that a PEG stopper is attached to the SNP surface in a mushroom conformation and that the particles are dispersed in aqueous solution, the 
average diameter of the PEG coil ( $\xi_{\text {mushroom }}$ ) assembled onto the SNP surface can be calculated by $\xi_{\text {mushroom }}=0.76 \times\left(\mathrm{M}_{\mathrm{w}(\mathrm{PEG})}\right)^{0.5}$ with $\mathrm{M}_{\mathrm{w}(\mathrm{PEG})}$ the molecular weight of the PEG chain and the area of SNP covered by the polymer with $A_{\text {mushroom }=} \Pi \times\left(\xi_{\text {mushroom }} / 2\right)^{2} .{ }^{32}$ Taking into account that the SNPs are surrounded by PEG, we estimate that the average SNP diameter in water, compared to SNPs made using Np-PEG ${ }_{18}$, would increase by 6 $\mathrm{nm}$ for SNPs assembled with Np-PEG 111 and up to $17 \mathrm{~nm}$ for SNPs assembled with Np$\mathrm{PEG}_{464}$. Although the data shown in Figure 4.4 do not contradict, these calculations, the size distributions are too broad to give a firm experimental confirmation.

Table 4.1: Polymer diameter and area of PEG with different PEG length assembled in mushroom conformation onto the particles surfaces.

\begin{tabular}{|c|c|c|c|}
\hline PEG derivative & $\mathrm{M}_{\mathrm{W}}$ PEG chain $(\mathrm{g} / \mathrm{mol})$ & $\xi_{\text {mushroom }}(\mathrm{nm})$ & $A_{\text {mushroom }}\left(\mathrm{nm}^{2}\right)$ \\
\hline $\mathrm{Np}-\mathrm{PEG}_{3}$ & 133 & 0.9 & 0.6 \\
\hline $\mathrm{Np}-\mathrm{PEG}_{18}$ & 801 & 2.2 & 3.6 \\
\hline Np-PEG ${ }_{111}$ & 4884 & 5.3 & 22.2 \\
\hline $\mathrm{Np}-\mathrm{PEG}_{464}$ & 20416 & 10.9 & 92.6 \\
\hline
\end{tabular}

For the formation of SNPs with PEGs of longer chain lengths, the PEG chains require a larger surface area per chain, so less PEG chains are required to occupy the SNP surface and to stabilize the assembly. From this, it can be expected that an increasing fraction of stopper remains unused, and creates a thermodynamic driving force for the formation of smaller (by core) SNPs. To support this, it is estimated from the binding constant of Np with the preformed $\mathrm{CB}[8] / \mathrm{MV}$ complex $\left((6.1 \pm 0.5) \times 10^{5} \mathrm{M}^{-1}\right)^{33}$ and the low building block concentration used for SNP formation $(0.7 \mu \mathrm{M})$, that maximally only $\sim 42 \%$ of the NpPEG molecules are assembled onto the SNP surface and the residual PEG chains are free in solution. This percentage decreases upon increasing the PEG chain length, indicating a lower number of PEG stopper molecules are attached onto the SNP surface. This could explain the increasing difference between DLS and SEM sizes indicated in Figure 4.4c. The SNPs prepared with the longest PEG chains show the largest loss of water upon drying, which is related to a higher hydration of the SNPs, a larger content of PEG and possibly a lower content of stopper.

Overall, since we do not observe a clear size increase or decrease in the formation of SNPs with different PEG lengths, we assume that both mechanisms could be playing a combined and counteracting role in the determination of the SNP size by self-assembly. Although the mushroom conformation of the longer PEG chains attached onto the SNP surface is larger, the SNP core shrinkage due to the occupation of more space by PEG could lead to a negligible size difference between the SNPs prepared with different PEG lengths. 
Upon close comparison between the composition and configuration of the SNPs, the often proclaimed effect of steric stabilization by monovalent Np-PEGs becomes obscure. Assuming that the SNPs are formed by $90 \%$ water and $10 \%$ organic material and that a single $\mathrm{CB}[8]$ has an intrinsic surface of $3 \mathrm{~nm}^{2},{ }^{34}$ a single $\mathrm{CB}[8]$ molecule is assembled approximately onto a SNP surface of $30 \mathrm{~nm}^{2}$. Taking into account that only $42 \%$ of the Np-PEG interacts with the CB[8]/MV complexes, this corresponds to a single Np-PEG polymer chain assembled onto $71 \mathrm{~nm}^{2}$ of SNP surface. By taking into account the mushroom conformational area occupied by the different Np-PEGs (Table 4.1), it is logical that only the SNPs prepared with Np-PEG 464 are formed by a shell in which the polymer chains feel each other and dense PEG coverage is ensured. Therefore only for these SNPS a lower number of PEG molecules might be assembled onto the SNP surface, whereas all other SNPs are formed by the same amount of Np-PEG chains, verifying the results shown in Figure 4.4. On the other extreme, Np-PEG 18 provides colloidal stability, but only about $5 \%$ of the SNP surface area is covered by PEG.

Overall, it can be assumed that the Np-PEGs with shorter PEG length provide only marginal polymer shielding of the SNPs. Therefore, we propose that the SNPs are additionally stabilized by a dynamic effect in which the Np-PEGs are hopping between the different positions available on the SNPs surface and thereby provide colloidal stabilization and prevent aggregation.

\subsubsection{Supramolecular nanoparticle formation with stoppers of different valencies}

To study the effect of the valency of the stopper on the SNP formation, the divalent $\mathrm{Np}_{2}$ $\mathrm{PEG}_{113}$ was synthesized and used for SNP formation. In accordance with the previous experiments, $\mathrm{Np}_{2}-\mathrm{PEG}_{113}, \mathrm{~Np}_{8}-\mathrm{PAMAM}, \mathrm{MV}$-PEI and $\mathrm{CB}[8]$ were mixed and SNP formation was evaluated by SEM and DLS after keeping the samples at room temperature for 2 and 7 days, respectively. It should be noted that, since two naphthol groups are grafted onto the divalent $\mathrm{Np}_{2}-\mathrm{PEG}_{113}$, only half of the molar concentration of the stopper was used for SNP formation, in comparison to the monovalent Np-PEGs, to keep the concentrations of host and guest moieties equimolar.

As observed by SEM (Figure 4.5), self-assembly of the supramolecular building blocks led to the formation of supramolecular particle-like assemblies for different dendrimerstopper ratios, but the shape of these SNPs was not as spherical as observed for SNPs prepared with Np-PEG 113 (see Chapter 3). This is justified by the absence of stable and reproducible DLS readings for SNPs prepared with $10 \% \mathrm{~Np}$ from $\mathrm{Np}_{8}$-PAMAM and the occurrence of heterogeneous structures by SEM for SNPs prepared with $20 \%$ and $30 \%$ $\mathrm{Np}$ from $\mathrm{Np}_{8}$-PAMAM. 

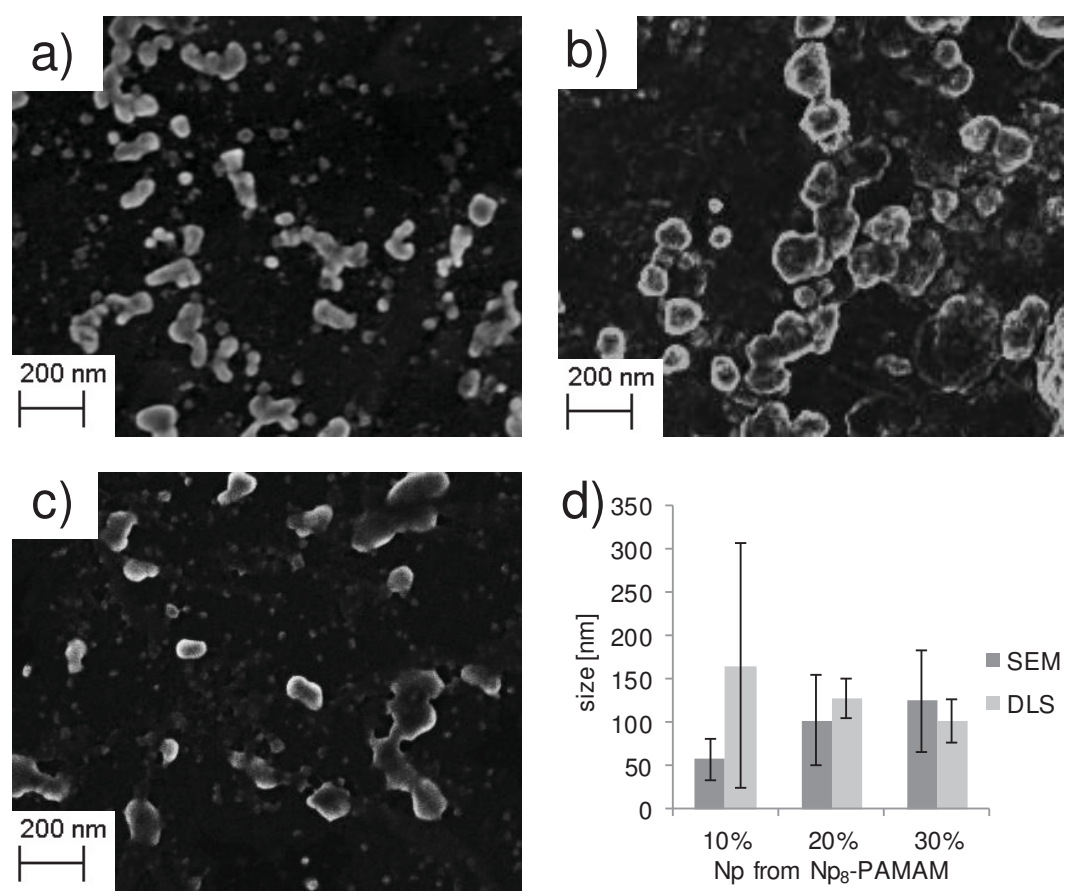

Figure 4.5: Size determination of SNPs prepared with $N p_{2}-P E G_{113}$ at RT: (a-c) SEM images after 7

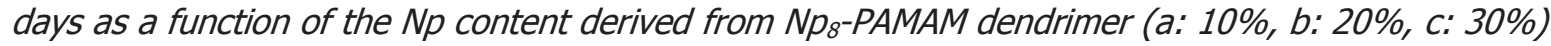
used during supramolecular assembly whilst keeping the concentration ratio of $C B[8]: M V: N p=$ 1:1:1. d) SNP diameter as measured by SEM ( $($ ) and DLS ( $)$.

To evaluate whether the SNP heterogeneity is a result of slow dynamics, time-dependent DLS measurements were carried out at RT and at $40^{\circ} \mathrm{C}$. As seen in Figure 4.6 , the observed hydrodynamic diameter of the SNPs containing $25 \% \mathrm{~Np}$ from $\mathrm{Np}_{8}$-PAMAM varied with temperature and with the valency of the Np-functionalized PEG. As shown in Chapter 3, the size of the SNPs prepared with Np-PEG ${ }_{111}$ is stabilized at $40^{\circ} \mathrm{C}$ within 4 to 6 h. However, prolonged heating (over $28 \mathrm{~h}$ ), led to SNP aggregation. In contrast, SNPs formed with $\mathrm{Np}_{2}-\mathrm{PEG}_{113}$ showed a reproducible size with low standard deviations after 12 to $14 \mathrm{~h}$ at $40^{\circ} \mathrm{C}$, and were stable during the whole monitoring time (40 h). Interestingly, at $\mathrm{RT}$, the samples prepared with $\mathrm{Np}_{2}-\mathrm{PEG}_{113}$ were not as consistent as the samples prepared at $40^{\circ} \mathrm{C}$ and the standard deviations remained relatively high, even $35 \mathrm{~h}$ after mixing. 


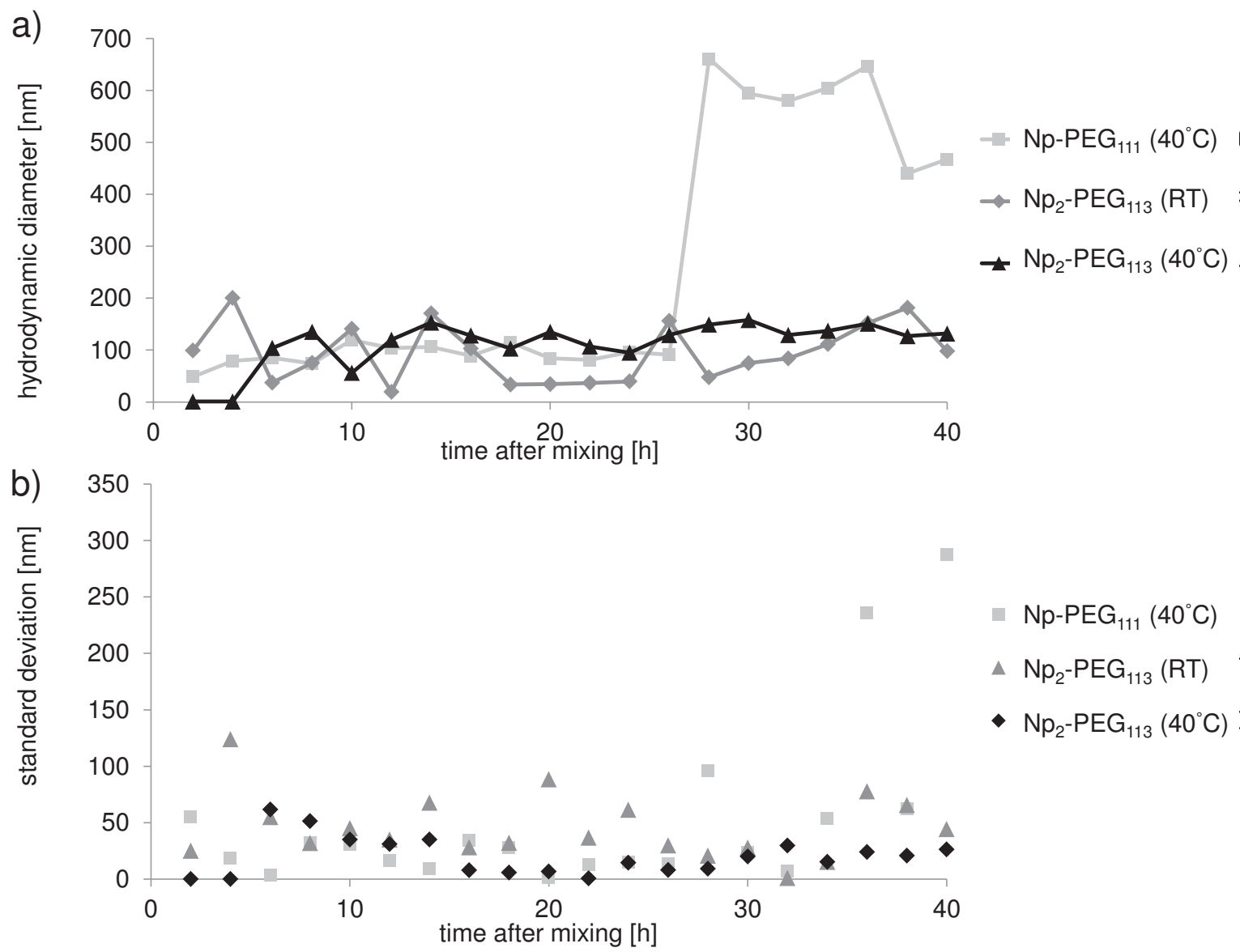

Figure 4.6: a) Hydrodynamic diameters of SNPs prepared with $N p-P E G_{111}$ or $N p_{2}-P E G_{113}$, consisting of $25 \% \mathrm{~Np}$ from $\mathrm{Np}_{8}-P A M A M$ measured by time-dependent $\mathrm{DLS}$ as a function of temperature (data averaged over four measurements) and b) corresponding standard deviations.

Subsequently, the size tunability of the SNPs formed with $\mathrm{Np}_{2}-\mathrm{PEG}_{113}$ was evaluated by preparing the SNP samples at $40^{\circ} \mathrm{C}$ instead of at RT. Hereto, the supramolecular building blocks were mixed and kept at $40^{\circ} \mathrm{C}$ for $10 \mathrm{~h}$ followed by $1 \mathrm{~d}$ at RT prior to characterization. As observed in Figure 4.7, more distinct and spherical SNPs were observed by SEM. Furthermore, SEM and DLS size determination show clearly the characteristic stoichiometric size control by varying the content of divalent and multivalent Np-bearing building blocks. In particular, by increasing the amount of $\mathrm{Np}$ from $\mathrm{Np}_{8}$-PAMAM from $10 \%$ to $30 \%$, while decreasing the amount of $\mathrm{Np}$ from $\mathrm{Np}_{2}-\mathrm{PEG}_{113}$ from $90 \%$ to $70 \%$ correspondingly, an increase in SNP size was observed from $60 \pm 10 \mathrm{~nm}$ to $86 \pm 16 \mathrm{~nm}$ by SEM and from $88 \pm 9 \mathrm{~nm}$ to $144 \pm 36 \mathrm{~nm}$ by DLS (Figure 4.7d). 

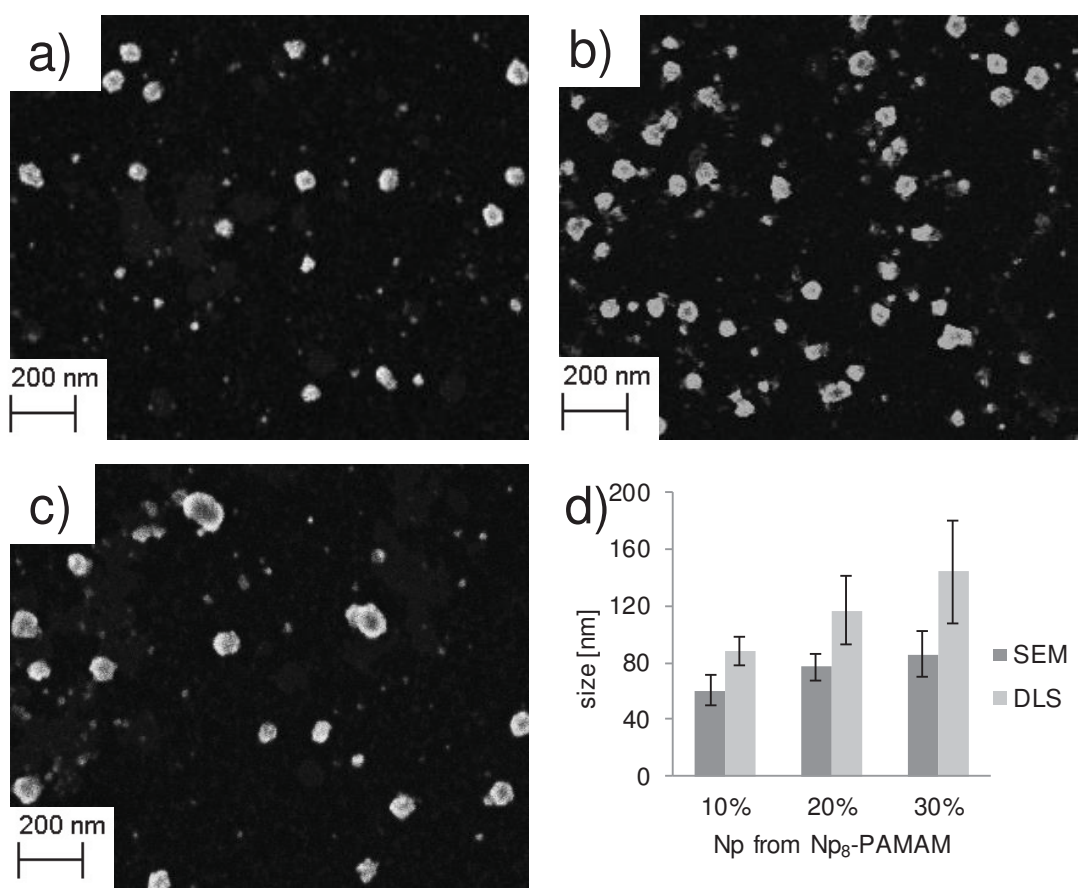

Figure 4.7: Size determination of SNPs prepared with $N p_{2}-P E G_{113}$ at 40 C: SEM images (a-c) as a function of the $N p$ content derived from Np $p_{8}$ PAMAM dendrimer (a: 10\%, b: 20\%, c: 30\%) used during supramolecular assembly while keeping the concentration ratio of CB[8]:MV:Np = 1:1:1. d) SNP diameter as measured by SEM ( () and DLS ( () .

Taken together, the time and temperature-dependent DLS measurements and the stoichiometry controlled SNP assembly show that an elevated temperature is required to form SNPs with distinct sizes and homogeneous shapes for SNPs prepared with the divalent $\mathrm{Np}_{2}-\mathrm{PEG}_{113}$ as the stopper. As described in Chapter 3, SNP self-assembly requires the dynamic disassembly and reassembly of the four supramolecular building blocks for well-defined SNP formation. By using a divalent instead of monovalent guest-modified PEG, this dynamic process is slowed down. This is in agreement with the observations and predictions that the overall dissociation rate of a guest molecule decreases by increasing its valency. ${ }^{35-38}$

For SNPs formed using the divalent stopper, the effective molarity (EM) - i.e. the probability of the second $\mathrm{Np}$ moiety of the divalent guest to find an available host molecule when it is already attached to the SNP surface via the first Np group determines the thermodynamic and kinetic multivalent stopper effects. We estimate the effective molarity on a SNP to be about $1 \mathrm{mM}$, which is similar to the $E M$ of a divalent adamantyl derivative binding to a cyclodextrin (CD) dimer. Although the size of $\mathrm{CB}[8]$ is comparable to $C D$, the value for a $C D$-coated surface $(100 \mathrm{mM})$ is likely to be too high compared to SNPs, as the SNP surface is not expected to be close packed (10\% pcking volume seems more realistic) and not all MV moieties are complexed with $\mathrm{CB}[8] .^{37}$ Together with the binding constant of $\left((6.1 \pm 0.5) \times 10^{5} \mathrm{M}^{-1}\right)^{33}$ for a Np unit binding to MV- 
$\mathrm{CB}[8]$, this leads to the estimation that the dissociation rate constant of the divalently bound stopper is 2-3 orders of magnitude lower than that of the monovalent one. This explains the slower formation observed at RT, although an exact timeframe for stable particle formation can currently not be established.

The time-dependent measurements at $40^{\circ} \mathrm{C}$ indicate that increasing the guest valency of the PEG stopper is slowing down of the dynamics with less than an order of magnitude at this temperature. It seems unlikely that such a small difference in temperature could cause a large change in binding affinity and effective molarity. Therefore, the limited change in the observed equilibration time probably indicates that the equilibration process is to a large extent dictated by the valency of the core dendrimer rather than the stopper, although the valency of the stopper has an unmistaken effect on the dynamics.

Notably, the SNPs formed by $10 \%$ to $30 \% \mathrm{~Np}$ from $\mathrm{Np}_{8}$-PAMAM and stabilized by the divalent $\mathrm{Np}_{2}-\mathrm{PEG}_{113}$ stopper are larger $(88 \pm 9 \mathrm{~nm}$ to $144 \pm 36 \mathrm{~nm}$ ) than the SNPs assembled with the monovalent Np-PEG ${ }_{111}(51 \pm 13 \mathrm{~nm}$ to $137 \pm 16 \mathrm{~nm})$. Yet, the surface coverage of the PEGs with the different valency does not explain the difference in observed SNP size. Even assuming that $\mathrm{Np}_{2}-\mathrm{PEG}_{113}$ completely binds to all $\mathrm{CB}[8]$ hosts assembled in the SNP shell, the SNP surface area is not completely covered by PEG and the individual polymer chains does not interact with each other, which is comparable to the SNPs prepared with Np-PEG 111 . More likely, the divalent character of $N p_{2}-P E_{113}$ causes the divalent guest to take part, to some extent, in the crosslinking of the supramolecular building blocks. This behavior has also observed for aggregation of CDfunctionalized gold NPs with a bis-adamantyl guest molecule in solution. ${ }^{39}$ In this way, use of the divalent stopper may lead to an increased SNP size.

\subsection{Conclusions}

SNPs stabilized by ternary charge transfer complexes between $\mathrm{CB}[8]$, MV and Np can be assembled using different Np-modified PEGs. No difference in formation and size tunability was observed for SNPs prepared with Np-PEG 18 , Np-PEG 111 or Np-PEG 464 , whereas no clear SNP formation was observed for SNPs prepared with the short Npfunctionalized tri(ethylene glycol). From a comparison of the PEG size and the expected coverage of the SNP surface with ternary complexes, interaction of the individual PEG chains is only expected for the highest PEG length. Possibly, the SNPs are stabilized by the dynamic assembly and reassembly of the monovalent Np-functionalized PEGs, whereas steric effects provide some additional steric bulk, thereby leading to colloidal stability of the SNPs. In contrast to the monovalent Np-PEGs, the use of the divalent $\mathrm{Np}_{2}$ PEG requires an increased temperature to form size-tunable SNPs with distinct sizes. The apparent slowing down of the formation kinetics is due to the slower dissociation rate constant of the stopper, but the overall formation dynamics is to a large extent controlled 
by the multivalent core components. In a larger context, a better understanding of the colloidal stability and assembly dynamics of supramolecular nanoparticles stabilized by guest-functionalized PEGs will be beneficial for the development of functional delivery vectors that can be used in therapeutics.

\subsection{Acknowledgements}

Mark Smithers is acknowledged for the SEM images.

\subsection{Experimental Section}

\subsubsection{Materials}

Starting materials for organic synthesis were obtained from Sigma-Aldrich and Nanocs and used as received. Octa-(E)-5-naphthol-pentanoic-acid poly(amido amine) dendrimer (generation 1) ( $\mathrm{Np}_{8}$-PAMAM), methyl viologen-functionalized poly(ethylene imine) (MV$\mathrm{PEI})$ and naphthalene poly(ethylene glycol) methyl ether $\left(\mathrm{Np}-\mathrm{PEG}_{111}\right)$ were synthesized as described in Chapter 3. Cucurbit[8]uril (CB[8]) was purchased from Strem Chemicals and its purity was assessed by microcalorimetric titration against paraquat. Deuterated solvents used for NMR spectroscopy were purchased from Cambridge Isotope and the water used for dialysis and SNP preparation was of MilliQ quality (Millipore, $R=18.2$ $\left.\mathrm{M} \Omega \mathrm{cm}^{-1}\right)$.

\subsubsection{Synthetic procedures}

$\underline{\text { 2-(2-(2-(Naphthalen-2-yloxy)ethoxy)ethoxy)ethanol }\left(\mathrm{Np}-\mathrm{PEG}_{3}\right)(\mathbf{1})}$

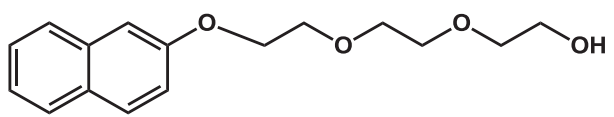

Under argon, 2-naphthol (0.52 $\mathrm{g}, 3.6 \mathrm{mmol})$ was dissolved in anhydrous THF $(10 \mathrm{~mL})$. At $0^{\circ} \mathrm{C}, \mathrm{NaH}$ ( $60 \%$ in mineral oil, $140 \mathrm{mg}, 3.6 \mathrm{mmol}$ ) was added and the solution was stirred $1 \mathrm{~h}$, while warming to room temperature. Subsequently, the solution was transferred under argon to a second flask charged with chloro-ethoxyethoxy-ethanol $(1.5 \mathrm{~g}, 8.95 \mathrm{mmol})$ in THF $(40 \mathrm{~mL})$. A trace of $\mathrm{KI}$ was added to promote Finkelstein halogen exchange and the solution was stirred at room temperature for $12 \mathrm{~h}$ under argon. The solvent was removed under reduce pressure and the residue dissolved in $30 \mathrm{~mL} \mathrm{CH} \mathrm{Cl}_{2}$. Unreacted chloro-ethoxy-ethoxy-ethanol was removed by extraction of the organic solution with $(6 \times 50 \mathrm{~mL}) 2 \mathrm{M} \mathrm{HCl}$ followed by brine $(30 \mathrm{~mL})$. The organic phase was dried with $\mathrm{MgSO}_{4}$ and the solvent removed under reduced pressure. Flash chromatography $\left(\mathrm{SiO}_{2}\right.$, gradient elution $\mathrm{CH}_{2} \mathrm{Cl}_{2}(100 \%)$ to $\left.\mathrm{CH}_{2} \mathrm{Cl}_{2} / \mathrm{MeOH}(98: 2)\right)$ gave Np$\mathrm{PEG}_{3}$ as a slightly yellow oil. Yield: $550 \mathrm{mg}, 55 \%$. ${ }^{1} \mathrm{H}-\mathrm{NMR}\left(400 \mathrm{MHz}, \mathrm{CDCl}_{3}\right): \delta 7.75-7.69$ $(\mathrm{q}, 3 \mathrm{H}, 3 \mathrm{ArH}) ; 7.43-7.39(\mathrm{t}, 1 \mathrm{H}, 1 \mathrm{ArH}) ; 7.33-7.29(\mathrm{t}, 1 \mathrm{H}, 1 \mathrm{ArH}) ; 7.18-7.12(\mathrm{~m}, 2 \mathrm{H}$, $2 \mathrm{ArH}) ; 4.23\left(\mathrm{t}, 2 \mathrm{H}, \mathrm{CH}_{2}\right) ; 3.91\left(\mathrm{t}, 2 \mathrm{H}, \mathrm{CH}_{2}\right) ; 3.73-3.67\left(\mathrm{~m}, 6 \mathrm{H}, 3 \mathrm{CH}_{2}\right) ; 3.6\left(\mathrm{t}, 2 \mathrm{H}, \mathrm{CH}_{2}\right)$. $\left.{ }^{13} \mathrm{C}-\mathrm{NMR}\left(100 \mathrm{MHz}^{\mathrm{CDCl}}\right)_{3}\right): \delta=156.65,134.49,129.46,129.08,127.66,126.78,126.39$, 
123.72, 118.96, 106.77, 72.56, 70.85, 70.36, 69.73, 67.35, 61.76. ESI-MS (m/z): calculated for $\left[\mathrm{C}_{16} \mathrm{H}_{20} \mathrm{O}_{4}\right]^{+}: 276.14$, found: $277.10(\mathrm{M}+\mathrm{H})^{+}$.

\section{$\underline{\mathrm{Np}-\mathrm{PEG}_{18}(\mathbf{2})}$}

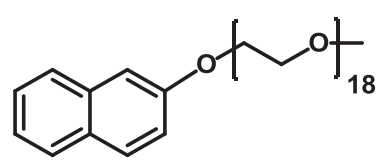

Under nitrogen, naphthol $(0.8 \mathrm{~g}, 5.6 \mathrm{mmol})$, tosyl poly(ethylene glycol) methyl ether $\left(\mathrm{M}_{\mathrm{w}} 900 \mathrm{~g} / \mathrm{mol}\right)(1 \mathrm{~g}, 1.11 \mathrm{mmol})$ and $\mathrm{K}_{2} \mathrm{CO}_{3}$ $(1.7 \mathrm{~g}, 5.6 \mathrm{mmol})$ were dissolved in anhydrous acetonitrile (40 $\mathrm{mL}$ ) and stirred at reflux for 5 days. The solvent was evaporated and the residue dissolved in $10 \mathrm{~mL} \mathrm{CH} \mathrm{CL}_{2}$. The excess of naphthol was removed by repeated precipitation in ice cold diethyl ether. The collected solid was dissolved in water, filtered and dialyzed against water for 5 days ( $M_{W}$ cut-off $550 \mathrm{~g} / \mathrm{mol}$ ). The aqueous solution was freeze-dried to obtain a dark brown solid. Yield: $550 \mathrm{mg}, 55 \%$. ${ }^{1} \mathrm{H}-\mathrm{NMR}(400 \mathrm{MHz}$, $\left.\mathrm{CDCl}_{3}\right): \delta=7.70-7.68(\mathrm{q}, 3 \mathrm{H}, 3 \mathrm{ArH}) ; 7.38(\mathrm{td}, 1 \mathrm{H}, 1 \mathrm{ArH}) ; 7.30(\mathrm{td}, 1 \mathrm{H}, 1 \mathrm{ArH}) ; 7.13(\mathrm{~m}$, $2 \mathrm{H}, 2 \mathrm{ArH}) ; 4.22\left(\mathrm{t}, 2 \mathrm{H}, \mathrm{CH}_{2}\right) ; 3.90\left(\mathrm{t}, 2 \mathrm{H}, \mathrm{CH}_{2}\right) ; 3.73\left(\mathrm{~m}, 2 \mathrm{H}, \mathrm{CH}_{2}\right) ; 3.64-3.52(\mathrm{~m}, 64 \mathrm{H}$, $\left.32 \mathrm{CH}_{2}\right) 3.35\left(\mathrm{~s}, 3 \mathrm{H}, \mathrm{CH}_{3}\right) .{ }^{13} \mathrm{C}-\mathrm{NMR}\left(100 \mathrm{MHz}, \mathrm{CDCl}_{3}\right): \delta=156.74,134.49,129.36$, $129.02,127.63,126.76,126.33,123.64,119.02,106.73,71.93,70.86,70.55,69.74$, $67.42,59.04$. ESI-MS (m/z): calculated for $\left[\mathrm{C}_{47} \mathrm{H}_{82} \mathrm{O}_{19}\right]^{+}: 950.55$, found: 945.57.

\section{$\underline{\mathrm{Np}-\mathrm{PEG}_{484}(3)}$}<smiles>COCCOc1ccc2ccccc2c1</smiles>

Under argon, 2-naphthoylchloride (20 mg, $0.104 \mathrm{mmol}$ ), 130 $\mathrm{mg}$ amino-poly(ethylene glycol) methyl ether ( $\mathrm{M}_{\mathrm{n}} 20.000$ $\mathrm{g} / \mathrm{mol}$ ) (130 $\mathrm{mg}, 0.013 \mathrm{mmol}$ ) and pyridine $(8.3 \mu \mathrm{L}, 0.104$ mmol) were stirred in $\mathrm{CH}_{2} \mathrm{Cl}_{2}(15 \mathrm{~mL})$ at room temperature for $48 \mathrm{~h}$. The solvent was evaporated under reduced pressure and the obtained solid precipitated in diethyl ether repeated times. The obtained solid was dissolved in $40 \mathrm{~mL}$ MilliQ water, filtered and dialyzed against water (disposable membrane molecular weight cut-off $5 \mathrm{kDa}$ ). Afterwards, the aqueous solution was filtered and a white fluffy solid was obtained after freeze-drying. Yield: $129 \mathrm{mg}, 98 \% .{ }^{1} \mathrm{H}-\mathrm{NMR}\left(400 \mathrm{MHz}, \mathrm{D}_{2} \mathrm{O}\right): \delta=8.39(\mathrm{~m}, 1 \mathrm{H}, 1 \mathrm{ArH})$; 8.08-8.00 (m, 3H, ArCH); 7.88-7.82 (m, $1 \mathrm{H}, \mathrm{ArH}) ; 7.62-7.70(\mathrm{~m}, 2 \mathrm{H}, 2 \mathrm{ArH}) ;$ 4.0-3.0 (m, $\left.940 \mathrm{H}, \mathrm{CH}_{2}\right) .{ }^{13} \mathrm{C}-\mathrm{NMR}\left(\mathrm{D}_{2} \mathrm{O}\right): \delta=69.56$. Maldi-MS $(\mathrm{m} / \mathrm{z})$ calculated for $\left[\mathrm{C}_{938} \mathrm{H}_{1863} \mathrm{NO}_{464}\right]^{+}$: $20581 \mathrm{~g} / \mathrm{mol}$, found: $20580 \mathrm{~g} / \mathrm{mol}$.

\section{2-(2-(2-(2-Bromoethoxy)ethoxy)ethoxy)naphthalene (4)}

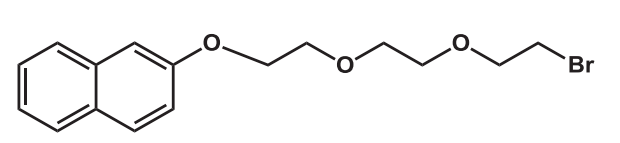

A solution of phosphorus tribromide $(0.40 \mathrm{~g}, 1.5$ $\mathrm{mmol})$ in toluene $(50 \mathrm{~mL})$ was added dropwise to a cooled $\left(0^{\circ} \mathrm{C}\right)$ solution of compound $\mathbf{1}(1.02 \mathrm{~g}, 3.7$ $\mathrm{mmol})$ in toluene $(50 \mathrm{~mL})$. The mixture was stirred overnight at room temperature. The solvent was removed under reduced pressure and the residue was partitioned between $\mathrm{CH}_{2} \mathrm{Cl}_{2}(50 \mathrm{~mL})$ and water $(50 \mathrm{~mL})$. The organic layer was washed with water $(3 \times 50$ 
$\mathrm{mL})$ and brine $(1 \times 50 \mathrm{~mL})$ and dried over $\mathrm{MgSO}_{4}$. The solvent was removed under reduced pressure and the residue was purified by column chromatography $\left(\mathrm{SiO}_{2}\right.$, $\left.\mathrm{CH}_{2} \mathrm{Cl}_{2} / \mathrm{MeOH}=99: 1\right)$ to give 2-(2-(2-(2-bromoethoxy)ethoxy) ethoxy)naphthalene as a colorless oil. Yield: $920 \mathrm{mg}, 73 \% .{ }^{1} \mathrm{H}-\mathrm{NMR}\left(\mathrm{CDCl}_{3}\right): \delta=7.75-7.69(\mathrm{q}, 3 \mathrm{H}, 3 \mathrm{ArH}) ; 7.44-$ $7.39(\mathrm{t}, 1 \mathrm{H}, 1 \mathrm{ArH}) ; 7.33-7.29(\mathrm{t}, 1 \mathrm{H}, 1 \mathrm{ArH}) ; 7.18-7.12(\mathrm{~m}, 2 \mathrm{H}, 2 \mathrm{ArH}) ; 4.25\left(\mathrm{t}, 2 \mathrm{H}, \mathrm{CH}_{2}\right)$; $3.94\left(\mathrm{t}, 2 \mathrm{H}, \mathrm{CH}_{2}\right) ; 3.77\left(\mathrm{t}, 2 \mathrm{H}, \mathrm{CH}_{2}\right) ; 3.75\left(\mathrm{~m}, 2 \mathrm{H}, \mathrm{CH}_{2}\right) ; 3.70\left(\mathrm{~m}, 2 \mathrm{H}, \mathrm{CH}_{2}\right) ; 3.46(\mathrm{t}, 2 \mathrm{H}$, $\left.\mathrm{CH}_{2}\right) \cdot{ }^{13} \mathrm{C}-\mathrm{NMR}\left(100 \mathrm{MHz}, \mathrm{CDCl}_{3}\right): \delta=156.73,134.49,129.40,129.04,127.65,126.77$, 126.37, 123.68, 119.02, 106.74, 71.28, 70.89, 70.63, 69.85, 67.43. ESI-MS (m/z): calculated for $\left[\mathrm{C}_{16} \mathrm{H}_{19} \mathrm{BrO}_{3}\right]: 338.05$ found: $339.22(\mathrm{M}+\mathrm{H})^{+}$.

\section{3,5-Bis(2-(2-(2-(naphthalen-2-yloxy)ethoxy)ethoxy)ethoxy)benzonitrile (5)}

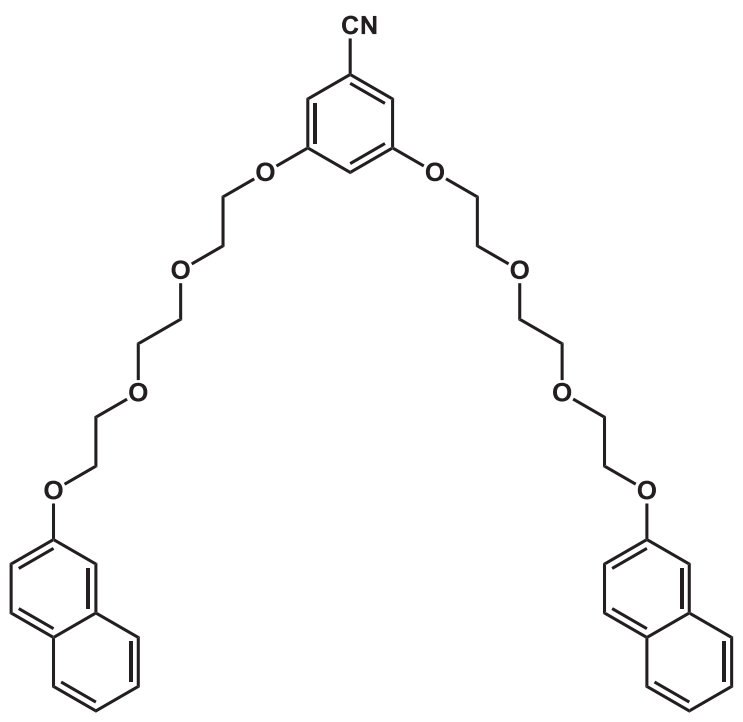
chromatography $\left(\mathrm{CH}_{2} \mathrm{Cl}_{2} / \quad \mathrm{MeOH}=98: 3\right)$ to give 3,5-bis(2-(2-(2-(naphthalen-2yloxy)ethoxy)ethoxy)ethoxy) benzonitrile as a colorless oil. Yield: $190 \mathrm{mg}, 50.3 \% .{ }^{1} \mathrm{H}-$ $\operatorname{NMR}\left(\mathrm{CDCl}_{3}\right): \delta=7.70-7.63(\mathrm{~m}, 6 \mathrm{H}, 6 \mathrm{ArH}) ; 7.36-7.34(\mathrm{t}, 2 \mathrm{H}, 2 \mathrm{ArH}) ; 7.29-7.2(\mathrm{t}, 2 \mathrm{H}$, $2 \mathrm{ArH}) ; 7.10-7.06$ (m, 4H, 4ArH); 6.67 (sd, 2H, 2ArH); 6.60 (sd, 1H, 1ArH); 4.19-4.17 (t, $\left.4 \mathrm{H}, 2 \mathrm{CH}_{2}\right) ; 3.99-3.97\left(\mathrm{t}, 4 \mathrm{H}, 2 \mathrm{CH}_{2}\right) ; 3.87-3.85\left(\mathrm{t}, 4 \mathrm{H}, 2 \mathrm{CH}_{2}\right) ; 3.77-3.75\left(\mathrm{~m}, 4 \mathrm{H}, 2 \mathrm{CH}_{2}\right)$; 3.73-3.68 (m, 8H, 4CH $) .{ }^{13} \mathrm{C}-\mathrm{NMR}\left(\mathrm{CDCl}_{3}\right): \delta=160.11,156.71,134.47,129.40,129.02$, $127.65,126.75,126.38,123.70,118.98,118.72,113.76,110.71,106.80,106.71,70.95$, 70.92, 69.84, 69.50, 67.91, 67.41. ESI-MS (m/z): calculated for $\left[\mathrm{C}_{39} \mathrm{H}_{41} \mathrm{NO}_{8}\right]$ : 651.28 found: $652.26(\mathrm{M}+\mathrm{H})^{+}$. 


\section{3,5-Bis(2-(2-(2-(naphthalen-2-yloxy)ethoxy)ethoxy)ethoxy)benzylamine}

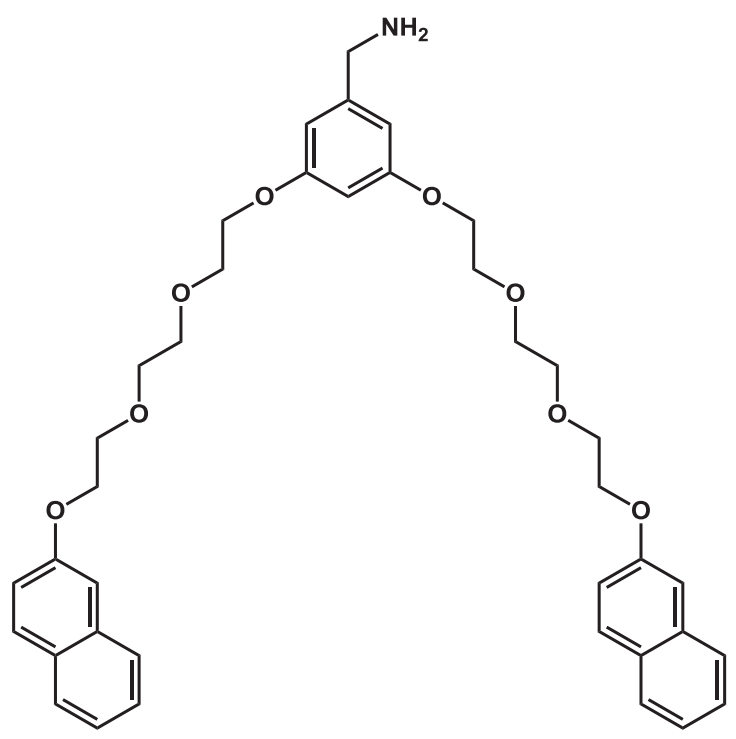

A suspension of compound 5 (136.2mg, $0.21 \mathrm{mmol}$ ) and a catalytic amount of Raney$\mathrm{Ni}$ was stirred under 10 bar $\mathrm{H}_{2}(\mathrm{~g})$ in $30 \mathrm{~mL}$ of $6 \mathrm{M} \mathrm{NH}_{3}$ in ethanol for $48 \mathrm{~h}$. The suspension was filtered over celite and washed with methanol. After evaporation of the solvent, the product was dissolved in chloroform. It was alkalize with $50 \mathrm{~mL}$ of $\mathrm{NaOH} 0.1 \mathrm{M}$. The water fraction was washed with chloroform $(3 \times 50 \mathrm{~mL})$. The organic fractions were combined and solvent evaporated under reduced pressure to give compound slightly greenish colored oil.

Yield: $113 \mathrm{mg}, 81 \%$, 80\% pure. ${ }^{1} \mathrm{H}-\mathrm{NMR}\left(\mathrm{CDCl}_{3}\right)$ : 7.67-7.63 (m, 6H, 6ArH); 7.36-7.34 (m, 2H, 2ArH); 7.29-7.22 (m, 2H, 2ArH); 7.10-7.06 (m, 4H, 4ArH); 6.50 (s, 2H, 2ArH); $6.37(\mathrm{~s}, 1 \mathrm{H}, 1 \mathrm{ArH}) ; 6.26(\mathrm{~s}, 1 \mathrm{H}, 1 \mathrm{ArH}) ; 4.17-4.12\left(\mathrm{~m}, 5.6 \mathrm{H}, \mathrm{CH}_{2} 2 \mathrm{CH}_{2}\right) ; 3.96-3.90(\mathrm{~m}$, $\left.4 \mathrm{H}, 2 \mathrm{CH}_{2}\right) ; 3.85-3.80\left(\mathrm{~m}, 4 \mathrm{H}, 2 \mathrm{CH}_{2}\right) ; 3.71-3.61\left(\mathrm{~m}, 12 \mathrm{H}, 4 \mathrm{CH}_{2}\right) .{ }^{13} \mathrm{C}-\mathrm{NMR}\left(\mathrm{CDCl}_{3}\right): \delta=$ $162.59,156.74,134.47,129.41,129.02,127.63,126.79,126.37,123.69,119.47$, $109.42,107.33,106.78,70.84,70.70,69.9,69.50,67.81,67.39,51.19$. ESI -MS (m/z): calculated for $\left[\mathrm{C}_{39} \mathrm{H}_{45} \mathrm{NO}_{8}\right]$ : 655.31 found: $656.68(\mathrm{M}+\mathrm{H})^{+}$.

\section{$\underline{\mathrm{Np}}_{2}-\underline{\mathrm{PEG}}_{113} \underline{(6)}$}

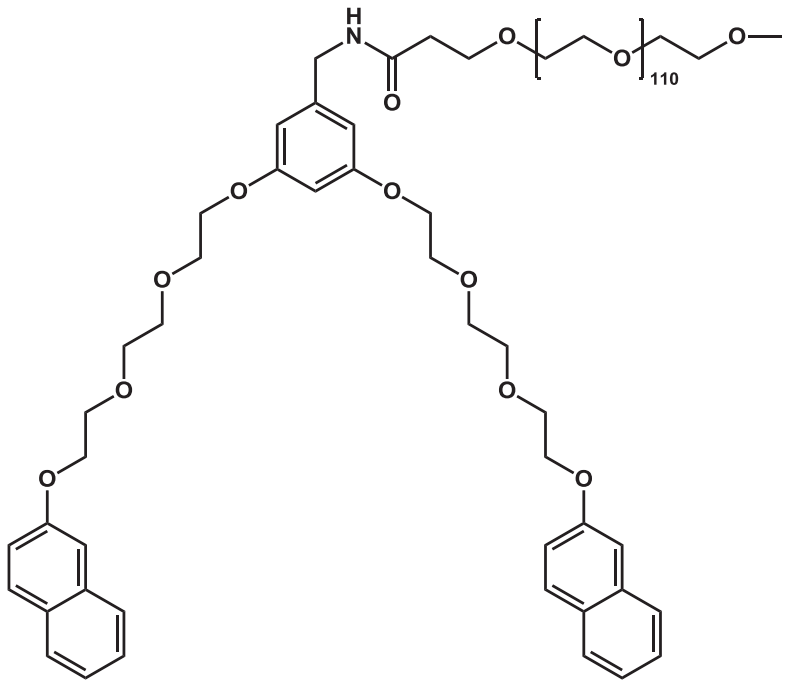

3,5-bis(2-(2-(2-(naphthalen-2-yloxy) ethoxy)ethoxy)ethoxy)benzylamine (100 $\mathrm{mg}, 0.152 \mathrm{mmol})$, triethylamine (15 mg, $21 \mu \mathrm{L}, 0.152 \mathrm{mmol}$ ) and methylpoly(ethylene glycol) $\mathrm{N}$ hydroxysuccinimide ester $\left(M_{w} \sim 5000\right.$ $\mathrm{g} / \mathrm{mol}$ ) (253 mg, $0.051 \mathrm{mmol}$ ) were dissolved in $\mathrm{CH}_{2} \mathrm{Cl}_{2}(30 \mathrm{~mL})$ under argon. The solution was stirred for 72 hours at room temperature. The solvent was removed under reduced pressure and the product purified by precipitation with diethyl ether and dialysis against water for 5 days. Yield: $265 \mathrm{mg}, 93 \%$. ${ }^{1} \mathrm{H}-\mathrm{NMR}\left(\mathrm{D}_{2} \mathrm{O}\right)$ : $7.72(\mathrm{~m}, 6 \mathrm{H}, 6 \mathrm{ArH}) ; 6.99(\mathrm{~m}, 2 \mathrm{H}, 2 \mathrm{ArH}) ; 6.87(\mathrm{~m}, 2 \mathrm{H}, 2 \mathrm{ArH}) ; 6.74(\mathrm{~m}, 2 \mathrm{H}, 2 \mathrm{ArH}) ; 6.61$ $(\mathrm{m}, 2 \mathrm{H}, 2 \mathrm{ArH}) ; 6.22(\mathrm{~s}, 2 \mathrm{H}, 2 \mathrm{ArH}), 5.98(\mathrm{~s}, 1 \mathrm{H}, 1 \mathrm{ArH}), 3.98\left(\mathrm{t}, 4 \mathrm{H}, 2 \mathrm{CH}_{2}\right), 3.78-3.10(\mathrm{~m}$, 
$\left.394 \mathrm{H}, 197 \mathrm{CH}_{2}\right) .{ }^{13} \mathrm{C}-\mathrm{NMR}\left(\mathrm{D}_{2} \mathrm{O}\right): \delta=69.56$. Maldi-MS (m/z) calculated for $\left[\mathrm{C}_{265} \mathrm{H}_{495} \mathrm{NO}_{121}\right]^{+}: 5631 \mathrm{~g} / \mathrm{mol}$, found: $5626 \mathrm{~g} / \mathrm{mol}$.

\subsubsection{Methods}

\section{Supramolecular nanoparticle assembly}

For the preparation of size-tunable supramolecular NPs utilizing $\mathrm{CB}[8]$ as supramolecular host, various aqueous solutions of the Np-PEGs (Np-PEG $, N p-P E G_{18}, N p-P E G_{111}, N p-$ $\mathrm{PEG}_{464}$ and $\mathrm{Np}_{2}-\mathrm{PEG}_{113}$ ) and different concentrations of AzO $-\mathrm{PAMAM}$ in DMSO were prepared. Additionally, aqueous solutions of MV-PEI $(0.336 \mu \mathrm{M})$ and $\mathrm{CB}[8](2.688 \mu \mathrm{M})$ were prepared and used for all SNP compositions. E.g. for preparing a solution having $20 \% \mathrm{~Np}$ entities derived from the aliphatic dendrimer and $80 \% \mathrm{~Np}$ entities derived from $\mathrm{Np}-\mathrm{PEG}_{18}, 1000 \mu \mathrm{L}$ MV-PEI was added to a previously prepared solution of $500 \mu \mathrm{L} \mathrm{Np}-$ PEG $_{18}(2.15 \mu \mathrm{M}), 20 \mu \mathrm{L} \mathrm{Np}$-PAMAM $(1.65 \mu \mathrm{M})$ and $500 \mu \mathrm{L} \mathrm{CB}[8](2.688 \mu \mathrm{M})$. These aliquots were mixed and kept at RT for 2 days before DLS and SEM analysis.

\section{SNP formation in time}

To compare the kinetics of the formation of SNPs in the presence of Np-PEG 111 and $\mathrm{Np}_{2}-$ $\mathrm{PEG}_{113}$, continuous DLS measurements were carried out for $40 \mathrm{~h}$ at RT and at $40^{\circ} \mathrm{C}$. Therefore the supramolecular building blocks were mixed using a formulation containing $25 \% \mathrm{~Np}$ from $\mathrm{Np}_{8}$-PAMAM, and DLS was started directly after mixing. The reported averaged SNP sizes and their standard deviations were obtained by averaging 4 data points measured over the course of $1 \mathrm{~h}$.

\subsubsection{Equipment}

\section{Dynamic light scattering (DLS)}

DLS experiments were performed with a Zetatrac and Nanotrac by Anaspec operating with a Microtrac FLEX Operating Software at $25^{\circ} \mathrm{C}$ using a laser wavelength of $780 \mathrm{~nm}$ at a scattering angle of $90^{\circ}$. The observed sizes and standard derivations of the SNPs were calculated by taking an average of 4 or 5 measurements. In total, particles with sizes ranging from 0.8 to $6400 \mathrm{~nm}$ were detected.

\section{Scanning electron microscopy (SEM)}

All SEM images were taken with a Carl-Zeiss high 1500 resolution scanning electron microscope. Therefore the aqueous SNP solutions were drop-cast on a Formvar coated copper TEM grid. The solution was dried and analyzed without further treatment of the samples. 


\section{NMR spectroscopy}

${ }^{1} \mathrm{H}$ and ${ }^{13} \mathrm{C}$ NMR spectra were recorded on Bruker $400 \mathrm{MHz}$ spectrometer. ${ }^{1} \mathrm{H}$ and ${ }^{13} \mathrm{C}$ chemical shift values, $400 \mathrm{MHz}$ and $100 \mathrm{MHz}$, are reported as $\delta$ using the residual solvent signal as internal standard.

\section{Mass spectrometry}

Mass analysis was done using the matrix-assisted laser desorption ionization (MALDI) and electrospray ionization using a Voyager DE-RP and a micromass LCT from Waters/Micromass, respectively.

\subsection{References}

1. Z.-Y. Zhou, N. Tian, J.-T. Li, I. Broadwell and S.-G. Sun, Chemical Society Reviews, 2011, 40, 4167-4185.

2. S. Guo and E. Wang, Nano Today, 2011, 6, 240-264.

3. H. Jiang, Small, 2011, 7, 2413-2427.

4. D. Jariwala, V. K. Sangwan, L. J. Lauhon, T. J. Marks and M. C. Hersam, Chemical Society Reviews, 2013, 42, 2824-2860.

5. Y. Xia, Nature Materials, 2008, 7, 758-760.

6. J. L. West and N. J. Halas, Annual Review of Biomedical Engineering, 2003, 5, 285-292.

7. L. Zhang, F. X. Gu, J. M. Chan, A. Z. Wang, R. S. Langer and O. C. Farokhzad, Clinical Pharmacologie Therapie, 2007, 83, 761-769.

8. C. Sun, J. S. H. Lee and M. Zhang, Advanced Drug Delivery Reviews, 2008, 60, 12521265.

9. I. Brigger, C. Dubernet and P. Couvreur, Advanced Drug Delivery Reviews, 2002, 54, 631-651.

10. Y. Min, M. Akbulut, K. Kristiansen, Y. Golan and J. Israelachvili, Nature Materials, 2008, 7, 527-538.

11. D. Walczyk, F. B. Bombelli, M. P. Monopoli, I. Lynch and K. A. Dawson, Journal of the American Chemical Society, 2010, 132, 5761-5768.

12. T. Cedervall, I. Lynch, S. Lindman, T. Berggård, E. Thulin, H. Nilsson, K. A. Dawson and

S. Linse, Proceedings of the National Academy of Sciences USA, 2007, 104, 2050-2055.

13. M. Mahmoudi, I. Lynch, M. R. Ejtehadi, M. P. Monopoli, F. B. Bombelli and S. Laurent, Chemical Reviews, 2011, 111, 5610-5637.

14. C. D. Walkey and W. C. W. Chan, Chemical Society Reviews, 2012, 41, 2780-2799.

15. S.-D. Li and L. Huang, Molecular Pharmaceutics, 2008, 5, 496-504.

16. R. A. Petros and J. M. DeSimone, Nature Reviews Drug Discovery, 2010, 9, 615-627.

17. R. Gref, Y. Minamitake, M. T. Peracchia, V. Trubetskoy, V. Torchilin and R. Langer, Science, 1994, 263, 1600-1603.

18. J. V. Jokerst, T. Lobovkina, R. N. Zare and S. S. Gambhir, Nanomedicine, 2011, 6, 715728.

19. B. Ballou, B. C. Lagerholm, L. A. Ernst, M. P. Bruchez and A. S. Waggoner, Bioconjugate Chemistry, 2003, 15, 79-86. 
20. S. D. Perrault, C. Walkey, T. Jennings, H. C. Fischer and W. C. W. Chan, Nano Letters, 2009, 9, 1909-1915.

21. C. D. Walkey, J. B. Olsen, H. Guo, A. Emili and W. C. W. Chan, Journal of the American Chemical Society, 2011, 134, 2139-2147.

22. K.-J. Chen, M. A. Garcia, H. Wang and H.-R. Tseng, in Supramolecular Chemistry, John Wiley \& Sons, Ltd, 2012.

23. Y. Ping, C. Liu, Z. Zhang, K. L. Liu, J. Chen and J. Li, Biomaterials, 2011, 32, 8328-8341.

24. M. E. Davis, J. E. Zuckerman, C. H. J. Choi, D. Seligson, A. Tolcher, C. A. Alabi, Y. Yen, J. D. Heidel and A. Ribas, Nature, 2010, 464, 1067-1070.

25. H. Wang, K.-J. Chen, S. Wang, M. Ohashi, K.-i. Kamei, J. Sun, J. H. Ha, K. Liu and H.-R. Tseng, Chemical Communications, 2010, 46, 1851-1853.

26. K.-J. Chen, L. Tang, M. A. Garcia, H. Wang, H. Lu, W.-Y. Lin, S. Hou, Q. Yin, C. K. F. Shen, J. Cheng and H.-R. Tseng, Biomaterials, 2012, 33, 1162-1169.

27. K.-J. Chen, S. M. Wolahan, H. Wang, C.-H. Hsu, H.-W. Chang, A. Durazo, L.-P. Hwang, M. A. Garcia, Z. K. Jiang, L. Wu, Y.-Y. Lin and H.-R. Tseng, Biomaterials, 2011, 32, 21602165.

28. Y. Liu, H. Wang, K.-i. Kamei, M. Yan, K.-J. Chen, Q. Yuan, L. Shi, Y. Lu and H.-R. Tseng, Angewandte Chemie International Edition, 2011, 50, 3058-3062.

29. S. Mishra, P. Webster and M. E. Davis, European Journal of Cell Biology, 2004, 83, 97111.

30. H. Wang, S. Wang, H. Su, K.-J. Chen, A. L. Armijo, W.-Y. Lin, Y. Wang, J. Sun, K.-i. Kamei, J. Czernin, C. G. Radu and H.-R. Tseng, Angewandte Chemie, 2009, 121, 44084412.

31. M. E. Davis, Molecular Pharmaceutics, 2009, 6, 659-668.

32. S. J. Budijono, B. Russ, W. Saad, D. H. Adamson and R. K. Prud'homme, Colloids and Surfaces A: Physicochemical and Engineering Aspects, 2010, 360, 105-110.

33. U. Rauwald, F. Biedermann, S. p. Deroo, C. V. Robinson and O. A. Scherman, Journal of Physical Chemistry B, 2010, 114, 8606-8615.

34. J. Lagona, P. Mukhopadhyay, S. Chakrabarti and L. Isaacs, Angewandte Chemie International Edition, 2005, 44, 4844-4870.

35. J. Huskens, A. Mulder, T. Auletta, C. A. Nijhuis, M. J. W. Ludden and D. N. Reinhoudt, Journal of the American Chemical Society, 2004, 126, 6784-6797.

36. A. Gomez-Casado, H. H. Dam, M. D. Yilmaz, D. Florea, P. Jonkheijm and J. Huskens, Journal of the American Chemical Society, 2011, 133, 10849-10857.

37. A. Perl, A. Gomez-Casado, D. Thompson, H. H. Dam, P. Jonkheijm, D. N. Reinhoudt and J. Huskens, Nature Chemistry, 2011, 3, 317-322.

38. A. Mulder, T. Auletta, A. Sartori, S. Del Ciotto, A. Casnati, R. Ungaro, J. Huskens and D. N. Reinhoudt, Journal of the American Chemical Society, 2004, 126, 6627-6636.

39. O. Crespo-Biel, A. Jukovic, M. Karlsson, D. N. Reinhoudt and J. Huskens, Israel Journal of Chemistry, 2005, 45, 353-362. 


\title{
Chapter 5
}

\section{Dual Stimuli-Responsive Self-Assembled Supramolecular Nanoparticles}

\begin{abstract}
Supramolecular nanoparticles (SNPS) encompass multiple copies of different building blocks brought together by specific noncovalent interactions. The inherently multivalent nature of these systems allows control of their size as well as their assembly and disassembly, thus offering promising potential as biomedical delivery vehicles. In this chapter, dual responsive SNPs have been based on the ternary host-guest complexation between cucurbit[8]uril (CB[8]), a methyl viologen (MV) polymer, and mono- and multivalent azobenzene (Azo)-functionalized molecules. UV switching of the Azo groups led to fast disruption of the ternary complexes, but to a relatively slow disintegration of the SNPs. Alternating UV and Vis photoisomerization of the Azo groups led to fully reversible SNP disassembly and reassembly. SNPs were only formed with the Azo moieties in the trans and the MV units in the oxidized states, respectively, thus constituting a supramolecular AND logic gate.
\end{abstract}




\subsection{Introduction}

The self-assembly of molecules into higher ordered supramolecular structures constitutes a powerful paradigm for the design of novel materials such as nanoparticles, ${ }^{1-3}$ hydrogels ${ }^{4-6}$ and polymers. ${ }^{7-9}$ These assemblies are held together by non-covalent weak interactions such as Van der Waals, hydrophobic, and electrostatic interactions. More robust host-guest interactions, for example, the inclusion of hydrophobic guests in cyclodextrins $(C D)^{10,11}$ or the inclusion of amines and hydrophobic guest molecules into cucurbit[n]urils (CB[n]), ${ }^{12}$ have been studied extensively in the last decade.

Among these systems, host-guest-based supramolecular nanoparticles (SNPs) currently receive significant interest. ${ }^{1-3}$ The self-assembly of small building blocks offers size control and colloidal stability by an interplay between multivalent and monovalent interactions. Their drug encapsulation properties and the easy implementation of targeting ligands make these assemblies promising candidates as site-selective biomedical delivery vectors. ${ }^{1,3}$

Although a variety of external controls has been used for the triggered release of proteins and cells from surfaces, ${ }^{13,14}$ RNA from micelles, ${ }^{15}$ and DNA from hydrogels ${ }^{16}$ and electrostatic delivery vectors, ${ }_{1}^{17}$ only chemical reduction ${ }^{18}$ as well as magnetic field ${ }^{19}$ have been used as specific triggers for the release of drugs encapsulated into SNPs.

Azobenzene (Azo) is known to bind to $a$ and $\beta-C D$ in the stable trans form. Upon irradiation with light at a wavelength shorter than $360 \mathrm{~nm}$ it undergoes a conversion to the unstable, bulkier cis form, which is released from the CD cavity. This phenomenon has been used to build photo-responsive supramolecular vesicles, ${ }_{1}^{20}$ mesoporous silica nanoparticles ${ }^{21,22}$ and hydrogels, ${ }^{23}$ which can bind and release DNA, drugs and proteins, respectively. In contrast, thermal trans-cis isomerization was used to fabricate an amine displacement assay driven by the complexation of Azo by cucurbit[7]uril. ${ }^{24}$ Recently, the formation of a ternary light-responsive host-guest complex between Azo, methyl viologen (MV) and cucurbit[8]uril (CB[8]) has been reported. ${ }^{25,26}$ Upon UV light irradiation, the cisAzo was formed, thus disrupting the heteroternary complex between $\mathrm{CB}[8]$, MV and Azo. Using this photo-induced mechanism, surface wettability changes ${ }^{25}$ as well as the polymerization of supramolecular building blocks ${ }^{26}$ could be triggered. Although so far uninvestigated, this system holds great promise as a reversible supramolecular glue for the controlled assembly and disassembly of supramolecular nanoparticles.

In Chapter 3, we have shown the formation of SNPs with controllable particle sizes, by employing a ternary charge-transfer complex between $\mathrm{CB}[8]$, MV and naphtholfunctionalized components. Here, we report a fully reversible and dual responsive SNP system using Azo building blocks, in which SNP assembly and disassembly can be 
switched reversibly in multiple cycles by photoswitching of Azo, and irreversibly by chemical reduction of the MV units. Based on the ternary host-guest complex formation between $\mathrm{CB}[8]$, MV-polymer and mono- and multivalent Azo-functionalized guest molecules, SNPs with controllable particle sizes are formed. As schematically shown in Scheme 1, the disintegration of the previously formed SNPS is studied upon the photochemical conversion from trans to cis-Azo induced by UV light irradiation $(\lambda<400$ $\mathrm{nm}$ ). Isomerization back to trans-Azo is induced by visible light and is expected to lead to full restoration of the SNPs. Additionally, the disassembly of the SNPs by chemical reduction has been investigated as well.

a)
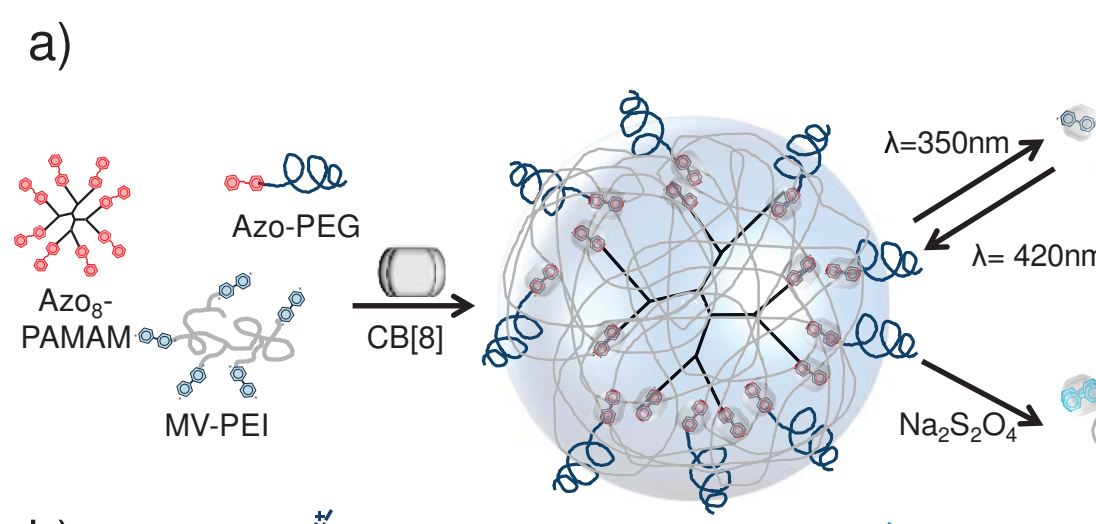

b)
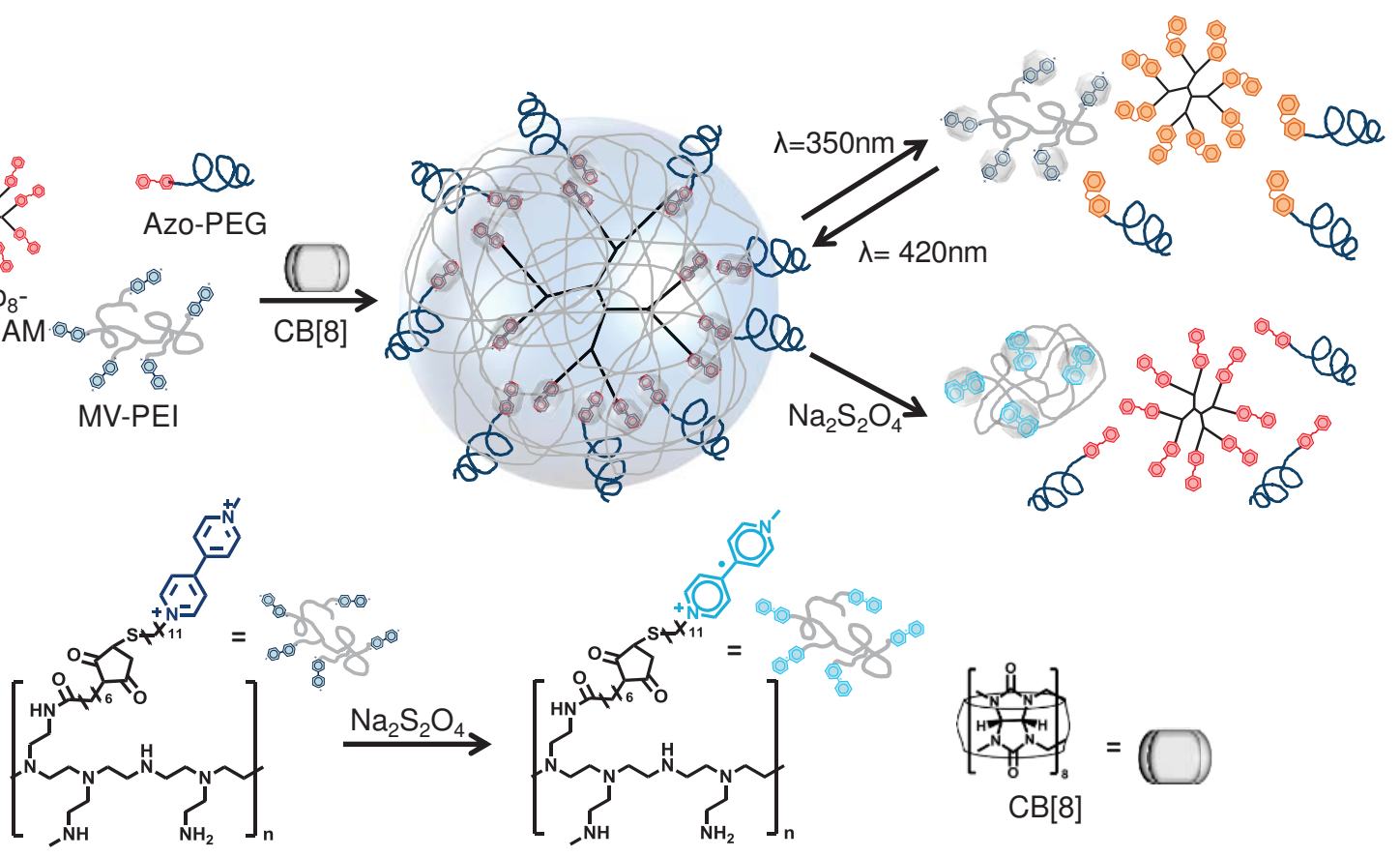

MV-PEI
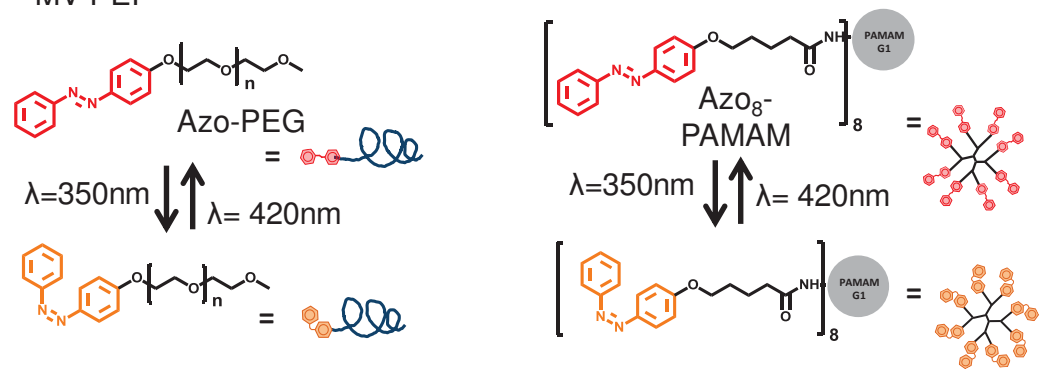

Scheme 5.1: a) Schematic presentation of the supramolecular nanoparticle (SNP) self-assembly and triggered disassembly mediated by formation and disruption of the ternary complex of cucurbit[8]uril (CB[8]), methyl viologen (MV) and azobenzene (Azo) moieties. b) Supramolecular host (CB[8]) and responsive guest molecules involved in SNP formation: azobenzene-poly(ethylene glycol) (Azo-PEG), methyl viologen-poly(ethylene imine) (MV-PEI) and Azos-poly(amidoamine) dendrimer $\left(\mathrm{AzO}_{8}-\mathrm{PAMAM}\right)$. 


\subsection{Results and Discussion}

\subsubsection{Supramolecular nanoparticle formation in aqueous solution}

SNP formation required the synthesis of the appropriate multi- and monovalent building blocks. To this end, Azo-terminated poly(amidoamine) dendrimer of generation 1 ( $\mathrm{AzO}_{8}{ }^{-}$ PAMAM) was synthesized to act as the multivalent photoresponsive supramolecular crosslinker in the core of the particles. Therefore, methyl-6-bromohexanoate was grafted onto $\mathrm{p}$-(phenylazo)phenol to prepare methyl (E)-5-(4(phenyldiazenyl)phenoxy)pentanoate. After deprotection, the carboxylic acid was activated using pentafluorophenol and the product purified by column chromatography. The fully Azo-functionalized PAMAM dendrimer was obtained after reacting perfluorophenyl (E)-5-(4-(phenyldiazenyl)phenoxy)pentanoate with the amine-terminated poly(amidoamine) dendrimer in DMF. In the presence of the previously reported MVsubstituted poly(ethylene imine) (MV-PEI, with a degree of substitution of 4 MV units per polymer chain) and $\mathrm{CB}[8]$, this Azo-dendrimer forms multiple ternary complexes in aqueous solution. To provide colloidal stability and size control of the formed SNPs, monovalent, Azo-functionalized poly(ethylene glycol) (Azo-PEG, $M_{W} 5000 \mathrm{~g} / \mathrm{mol}$ ) was prepared by the condensation of poly(ethylene glycol) monomethyl ether tosylate with phenyldiazobenzene in acetonitrile. Comparable to the SNP formation strategy reported in Chapter 3, the monovalent Azo-PEG competes with the multivalent $\mathrm{AzO}_{8}$-PAMAM for binding to $\mathrm{CB}[8]$, leading to termination of the assemblies and resulting in size-controlled SNP formation. The Azo-PEG also facilitates water solubility of the assembled SNPs by exposure of the PEG chains at the outside of the SNPS.

Size-controlled particle self-assembly was observed by mixing the four supramolecular blocks, i.e. mono- and multivalent Azo, multivalent MV-PEI, and $\mathrm{CB}[8]$, involved in ternary complex formation in varying ratios. To a mixture of $\mathrm{AzO}_{8}$-PAMAM dissolved in DMSO, aqueous CB[8] and aqueous Azo-PEG, a solution of MV-PEI was added subsequently. Successful size tuning was observed (Figure 5.1 ) by varying the ratio of the two Azo compounds, keeping the overall stoichiometry of $\mathrm{CB}[8]: \mathrm{MV}: \mathrm{Azo}=1: 1: 1$, and the mixtures were analyzed after two days using scanning electron microscopy (SEM) and dynamic light scattering (DLS). As shown in Figure 5.1e, the observed SNP size depends strongly on the fraction of $\mathrm{AzO}_{8}$-PAMAM used during particle self-assembly. By increasing the amount of Azo derived from $\mathrm{AzO}_{8}$-PAMAM from $10 \%$ to $30 \%$, while decreasing the amount Azo-PEG accordingly, the recorded particle size increased from $55 \mathrm{~nm}$ to $110 \mathrm{~nm}$ as measured by DLS and from $35 \mathrm{~nm}$ to $70 \mathrm{~nm}$ as observed by SEM. This discrepancy between the results from DLS and SEM is commonly observed and can be attributed to DLS reporting hydrodynamic diameters and to the loss of water during SEM sample preparation. Overall, the size increased monotonously and was approximately linearly dependent on the amount of $\mathrm{AzO}_{8}$-PAMAM up to $30 \%$. 

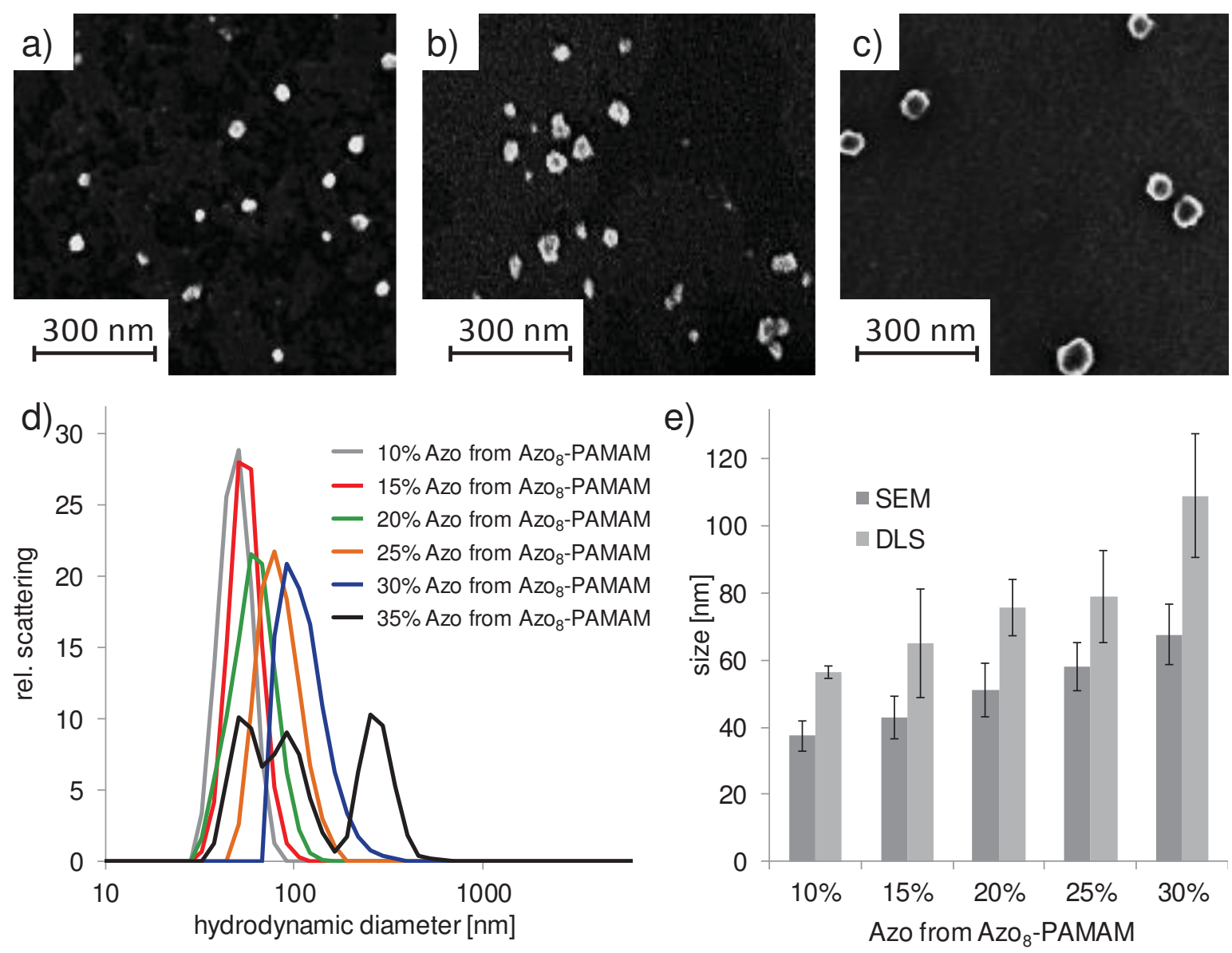

Figure 5.1: Size determination of SNPS prepared with different formulations: SEM images (a-c) of the resulting SNPS, as a function of the Azo content derived from Azos-PAMAM dendrimer (a: 10\%, b: 20\%, c: 30\%) used during supramolecular assembly (using CB[8]:MV:Azo = 1:1:1, with Azo deriving from Azos-PAMAM and Azo-PEG). d) DLS curves for the different SNP formulations. e) SNP diameter as measured by SEM ( () and DLS ( $)$.

DLS analysis further revealed that addition beyond 35\% $\mathrm{AzO}_{8}$-PAMAM led to uncontrolled particle growth yielding large aggregates with a high polydispersity (Figure 5.1d). This observation can be ascribed to the supramolecular competition between the multivalent dendrimers that reside in the core of the SNPs while the monovalent Azo-PEG assembles at the exterior of the SNPs. An increasing amount of the multivalent component allows an extended growth of the SNPs, yet a minimum amount of monovalent supramolecular stopper is apparently required for the formation of stable SNPs.

To prove the supramolecular specificity of the SNP self-assembly, SNP assembly was carried out in the absence of $\mathrm{CB}[8]$ and in the presence of $\mathrm{CB}[7]$ instead of $\mathrm{CB}[8]$. The smaller cucurbit[n]uril homologue does not sterically allow the formation of ternary complexes. As expected, no distinct particle formation was observed by DLS when MVPEI, Azo-PEG and Azo ${ }_{8}$-PAMAM (20\% Azo from Azo ${ }_{8}$-PAMAM) were mixed without CB[8] or when $\mathrm{CB}[7]$ was used (Figure 5.2 ). These observations show that ternary complex formation by $\mathrm{CB}[8]$ is essential for SNP formation. 

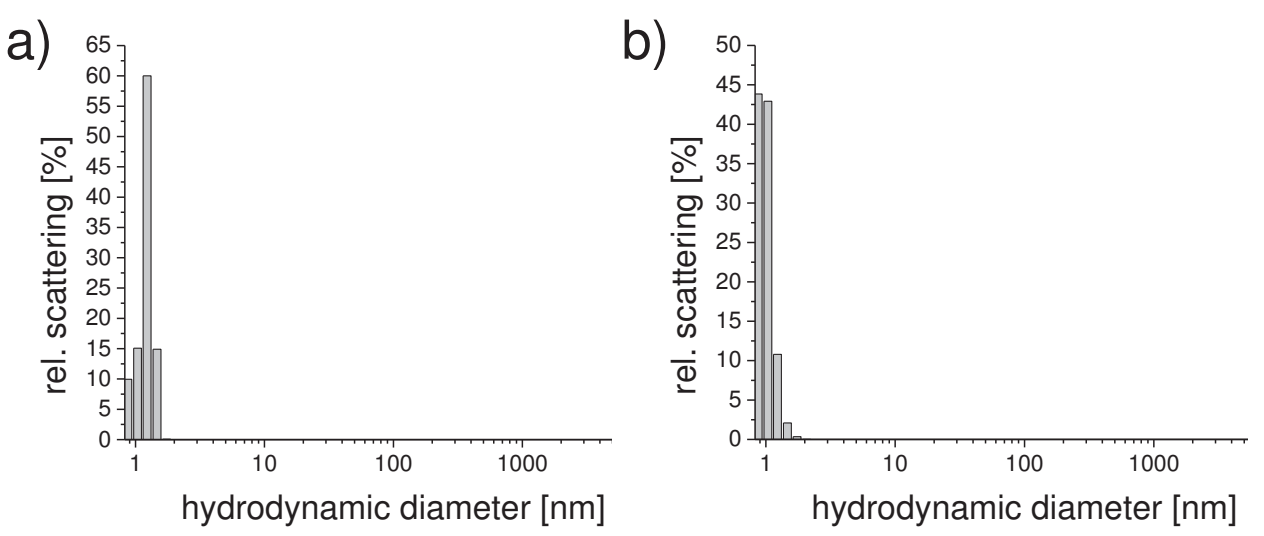

Figure 5.2: DLS analysis of solutions containing MV-PEI, Azo-PEG and Azo ${ }_{8}-P A M A M$ (20\% Azo from $A_{\left.2 O_{8}-P A M A M\right)}$ a) without $C B[8]$ and $b$ ) in the presence of $C B[7]$ instead of $C B[8]$

\subsubsection{Dual responsive supramolecular nanoparticle disassembly}

The light-induced disassembly and re-assembly of the particles was investigated making use of the photo-responsive heteroternary complex by conversion of trans to cis-Azo. UVVis spectroscopy (Figure 5.3) of the complex formed between $\mathrm{CB}[8]$, paraquat, and AzoPEG, as well as ${ }^{1} \mathrm{H}-\mathrm{NMR}$ spectroscopy (Figure 5.4) of Azo-PEG in $\mathrm{D}_{2} \mathrm{O}$ confirmed that $>90 \%$ of trans-Azo-PEG was converted into cis-Azo-PEG within $1 \mathrm{~min}$ after irradiating the aqueous solution with UV light $(\lambda<400 \mathrm{~nm})$. Time-dependent UV/Vis measurements show that the absorption band at $345 \mathrm{~nm}$ was restored after exposing the sample to ambient light for 20 min (Figure 5.3), which is indicative of cis-trans isomerization and concomitant re-formation of the ternary complex.

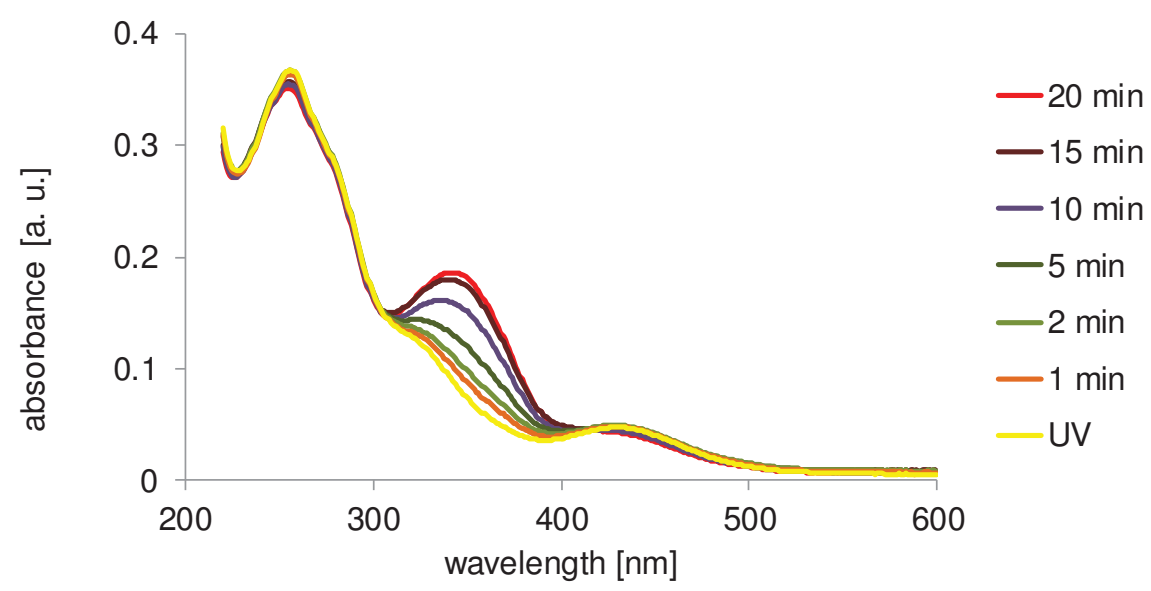

Figure 5.3: UV-Vis absorbance of CB[8]:paraquat:Azo-PEG: (1:1:0.95) before and after 1 min UV light irradiation as well as the time-dependent conversion of cis to trans-Azo-PEG under ambient light. 


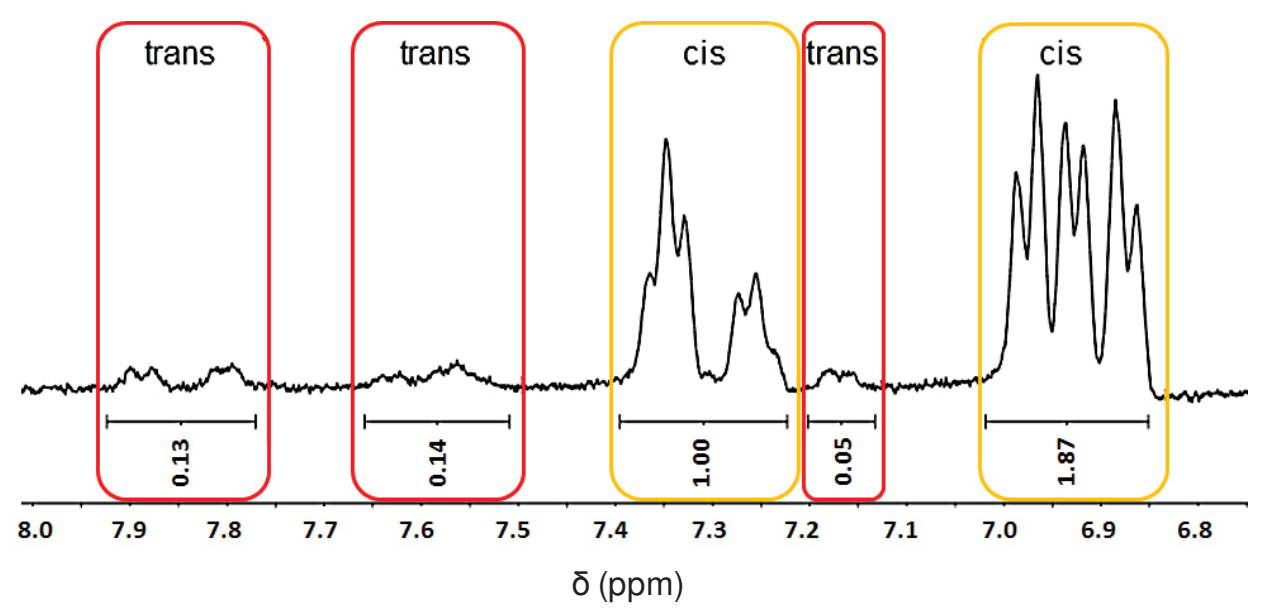

Figure 5.4: Partial ${ }^{1} \mathrm{H}$ NMR spectra of Azo-PEG in $\mathrm{D}_{2} \mathrm{O}$ after UV irradiation

Subsequently we irradiated previously prepared SNP solutions with UV light $(\lambda<400$ $\mathrm{nm}$ ) and followed the change in SNP size using DLS (Figure 5.5). After an initial UV exposure time of $1 \mathrm{~h}$, aggregated particles with a high polydispersity were observed (Figure 5.5). In contrast, full disintegration of the SNPs was observed after prolonging the irradiation time to $14 \mathrm{~h}$. This particle disassembly process is relatively slow in comparison to the $1 \mathrm{~min}$ switching time of Azo in the individual ternary complex as described above. This difference can be explained by i) an incomplete trans-to-cis isomerization of $\mathrm{Azo}$ and ii) a slow rearrangement of multivalently interacting building blocks. It has been reported, that the photostationary state of the UV irradiation of Azo contains up to $20 \%$ trans-Azo. ${ }^{20}$ Presumably, upon UV illumination, the cis-Azo-PEG is immediately expelled from the SNP shell, leading to exposure of the core. At the same time, the photostationary state ensures that the multivalent $\mathrm{AzO}_{8}$-PAMAM initially maintains some interactions through a few moieties inside the core, thus leading to significant clustering of the nanoparticles. As described in Chapter 3, the assembly of SNPs is slow- because of the slow rearrangement of the multivalently interacting components, therefore the disintegration of the remaining building blocks only takes place over the course of hours (Figure 5.5).

To verify the reversibility of the cis-to-trans switching that leads to the re-assembly of the individual components to nanoparticles, the disassembled SNP samples were kept in ambient light to induce the switching back to trans-Azo, thus enabling the re-formation of the ternary complex with MV and CB[8]. Complete SNP reassembly was observed after keeping the samples for $8 \mathrm{~h}$ under visible light (Figure 5.5). The SNP assemblydisassembly process was conducted several times by switching between UV and Vis light. Figure 5.6 shows the alternate switching for four complete cycles, and in all states a complete reformation $(\sim 116 \mathrm{~nm} \pm 16 \mathrm{~nm})$ or rupture $(\sim 2-4 \mathrm{~nm})$ of the SNPs was observed. These results confirm the successful development of a reversible, lightresponsive supramolecular nanoparticle system. 


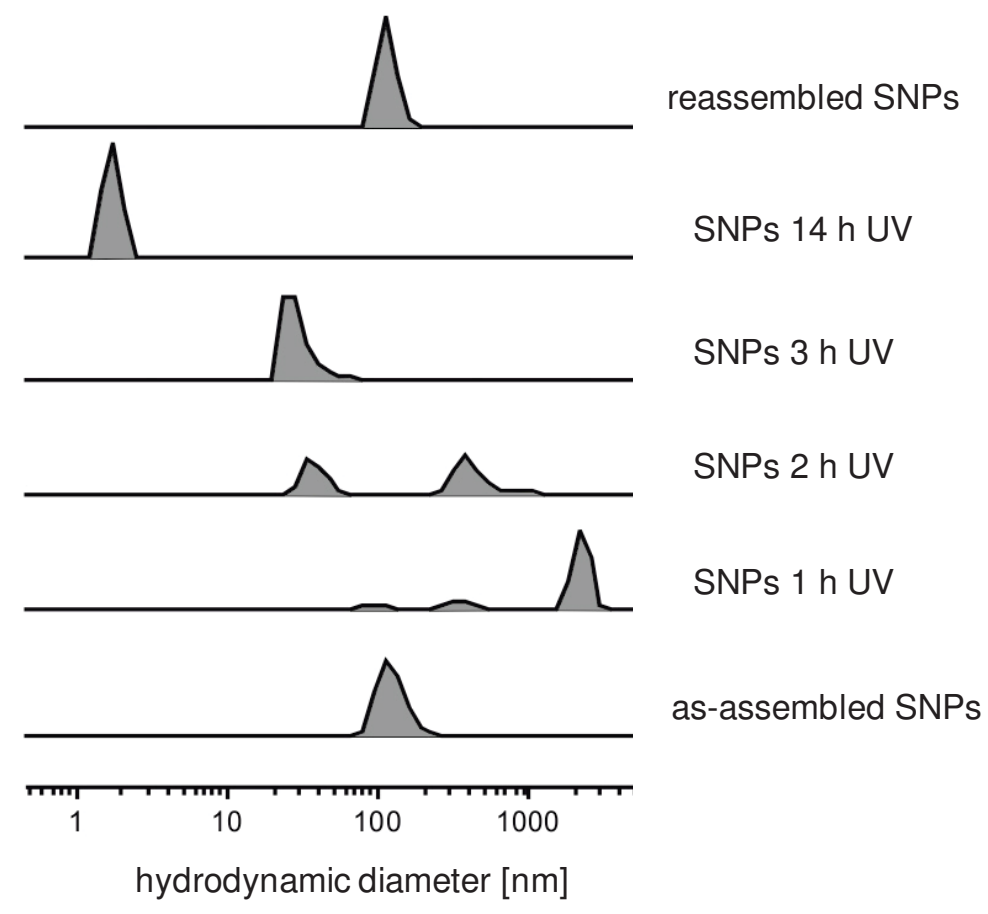

Figure 5.5: DLS size determination of: as-prepared SNPS (30\% Azo from Azos-PAMAM), and subsequently after 1 h UV light, after 2 h UV light, after 4 h UV light, after 6 h UV light, after 14 h UV light and after $8 \mathrm{~h}$ ambient light.

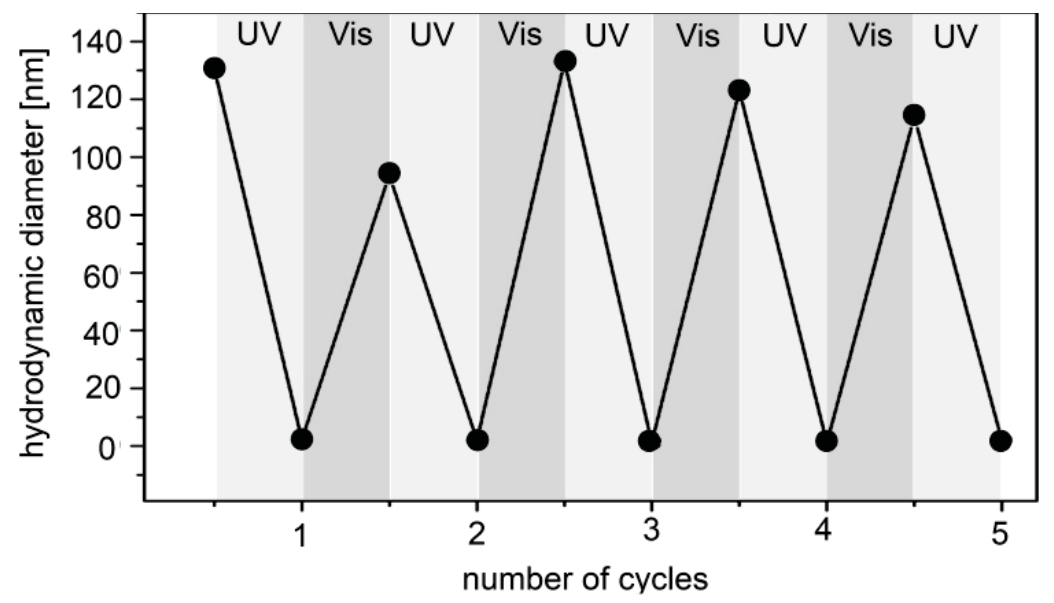

Figure 5.6: UV/Vis light-induced switching to trigger SNP assembly (SNPs prepared with $30 \%$ Azo from $\mathrm{AzO}_{8}-\mathrm{PAMAM}$ ) and disassembly for four consecutive cycles.

In contrast, no distinct particle reformation was observed for SNP samples kept in the dark for $6 \mathrm{~h}, 10 \mathrm{~h}$ and $14 \mathrm{~h}$ after UV irradiation (Figure 5.7). This shows, that the cis-totrans isomerization is driven by visible light irradiation, and that the thermal process plays a marginal role. 
a)

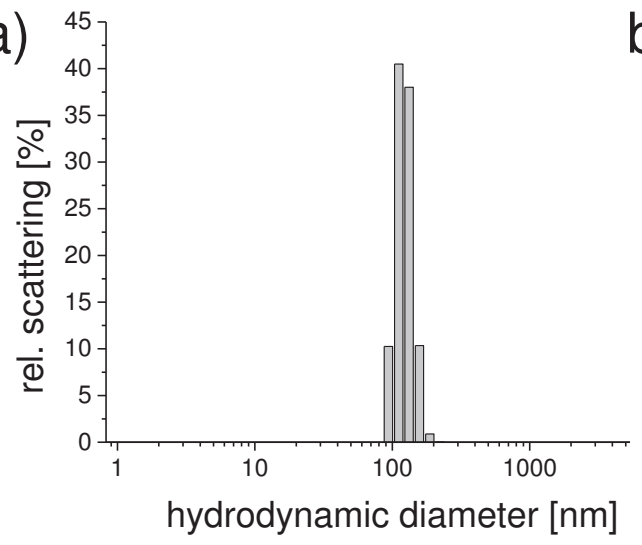

c)

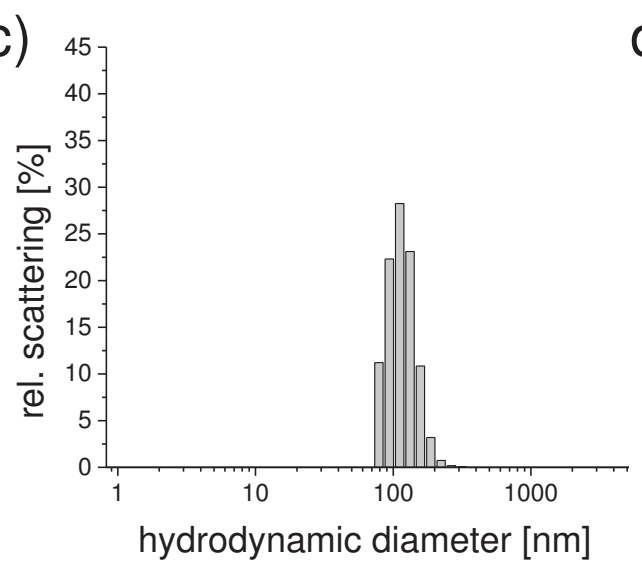

e)

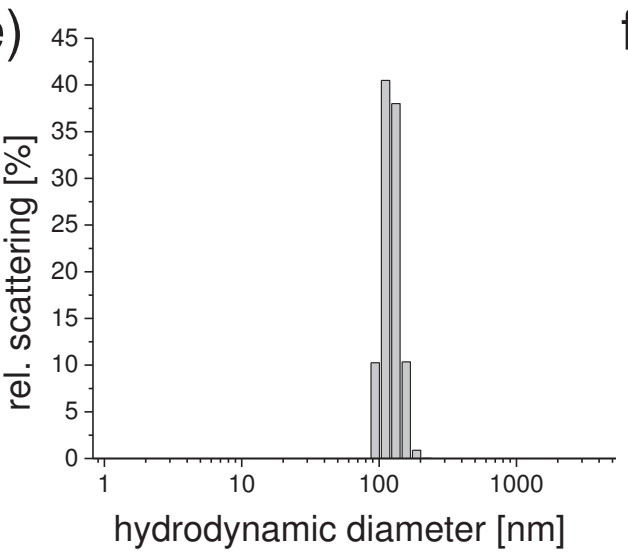

b)

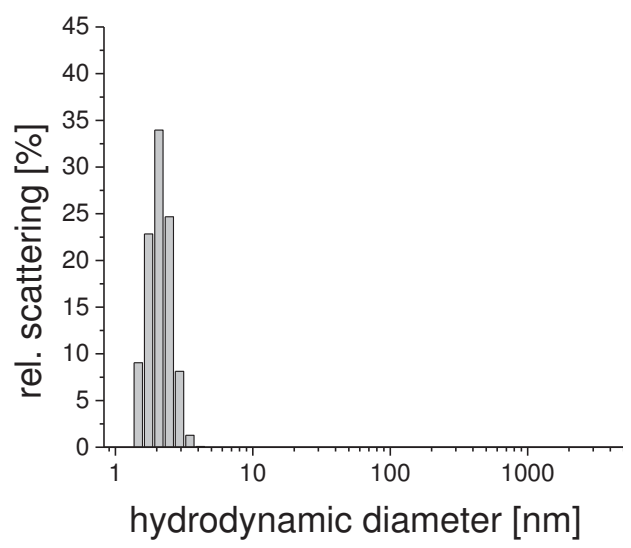

d)

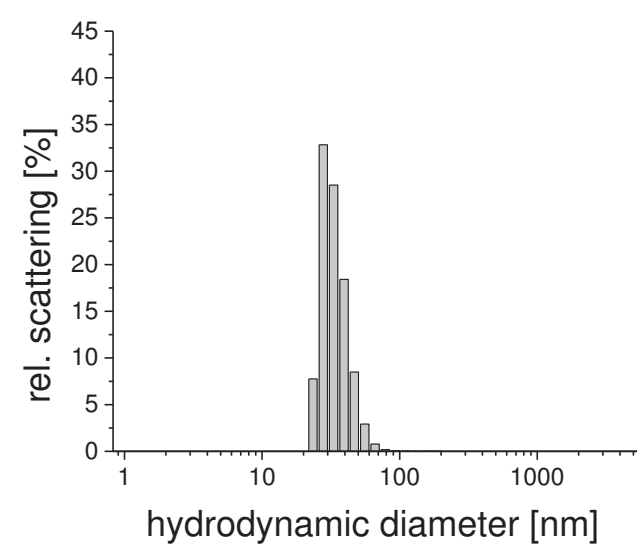

f)

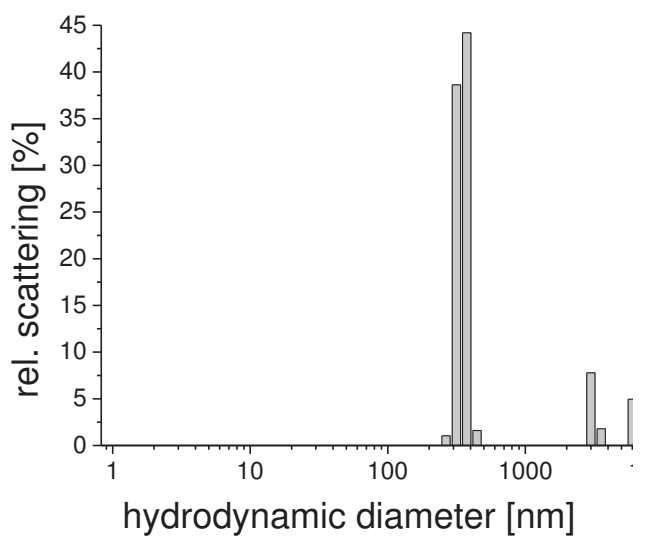

Figure 5.7: DLS analysis of three SNP samples (all prepared with 30\% Azo from Azo ${ }_{8}$-PAMAM): a) asassembled SNP sample 1 and b) and subsequently measured after 12 h UV irradiation followed by keeping in the dark for $6 h$; c) as-assembled SNP sample 2 and d) subsequently measured after $12 \mathrm{~h}$ UV irradiation followed by keeping in the dark for $10 \mathrm{~h}$; e) as-assembled SNP sample 3 and f) subsequently measured after 12 h UV irradiation followed by keeping in the dark for $14 \mathrm{~h}$.

The constitutive ternary supramolecular complex containing MV also results in the SNPs being susceptible to changes induced by chemical reduction of the MV moieties. Chemically triggered particle degradation was observed after the addition of the reducing agent $\mathrm{Na}_{2} \mathrm{~S}_{2} \mathrm{O}_{4}$. DLS analysis demonstrates the disappearance of particles with a size of about $110 \mathrm{~nm}$ and formation of small aggregates of about 2-4 nm (Figure 5.8) after 12 
h. This observation is in agreement with the supramolecular mechanism reported before. The addition of a chemical reductant leads to formation of $\mathrm{MV}^{+}$radical cations which are known to from stable homoternary complexes with $\mathrm{CB}[8],{ }^{27}$ thus causing the release of the Azo guest units (Scheme 5.1) and concomitant disassembly of the SNPs. ${ }^{25}$

a)

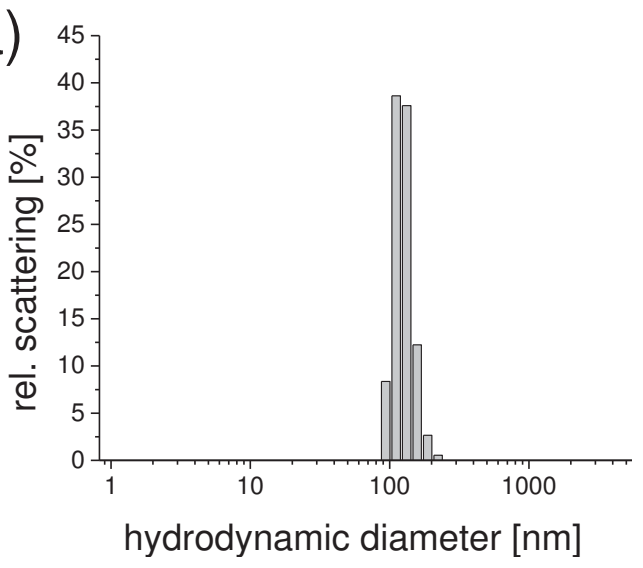

b)

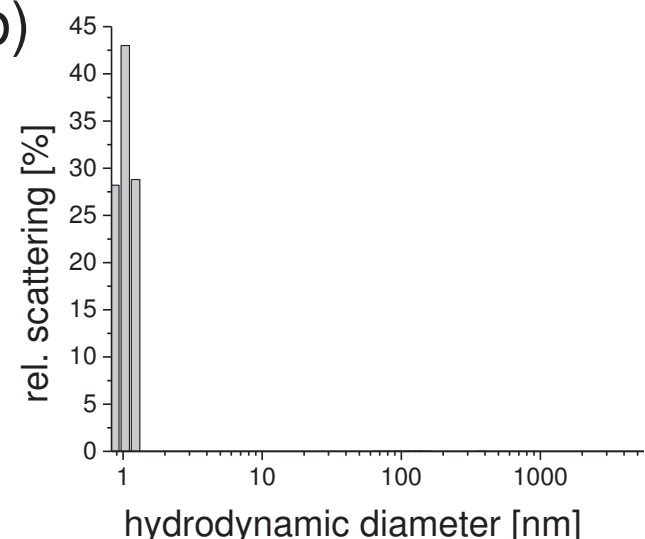

Figure 5.8: DLS analysis of SNPs prepared with 30\% Azo from Azo ${ }_{8}$-PAMAM a) as assembled, b) after $\mathrm{Na}_{2} \mathrm{~S}_{2} \mathrm{O}_{4}$ addition

To prove that particle reassembly requires both the trans-Azo as well as the dicationic MV species, the two particle disassembly processes were carried out consecutively. The formed SNPs (Figure 5.9a) were disassembled first by chemical reduction using $\mathrm{Na}_{2} \mathrm{~S}_{2} \mathrm{O}_{4}$, followed by UV irradiation. DLS studies show that the initially formed SNPs were neither observed after chemical reduction (Figure 5.9b), nor after UV light irradiation (Figure 5.9c). Furthermore, SNP reassembly was not observed after subsequent visible light treatment (Figure 5.9d), which can be ascribed to the still present homoternary complexes between $\mathrm{MV}^{+}$radical cations and $\mathrm{CB}[8]$.

In logic terms, the SNP system described here functions as an AND operator. Only when the Azo moiety is in the trans form and the MV moiety in the dicationic form, SNPs are formed (Figure 5.10). Switching either of them leads to inhibition of the particle formation process. Molecular systems representing logic operators have been shown and reviewed before. ${ }^{28}$ To our knowledge, this is the first example in which the result of the operation is visualized in the formation of supramolecular particles. 

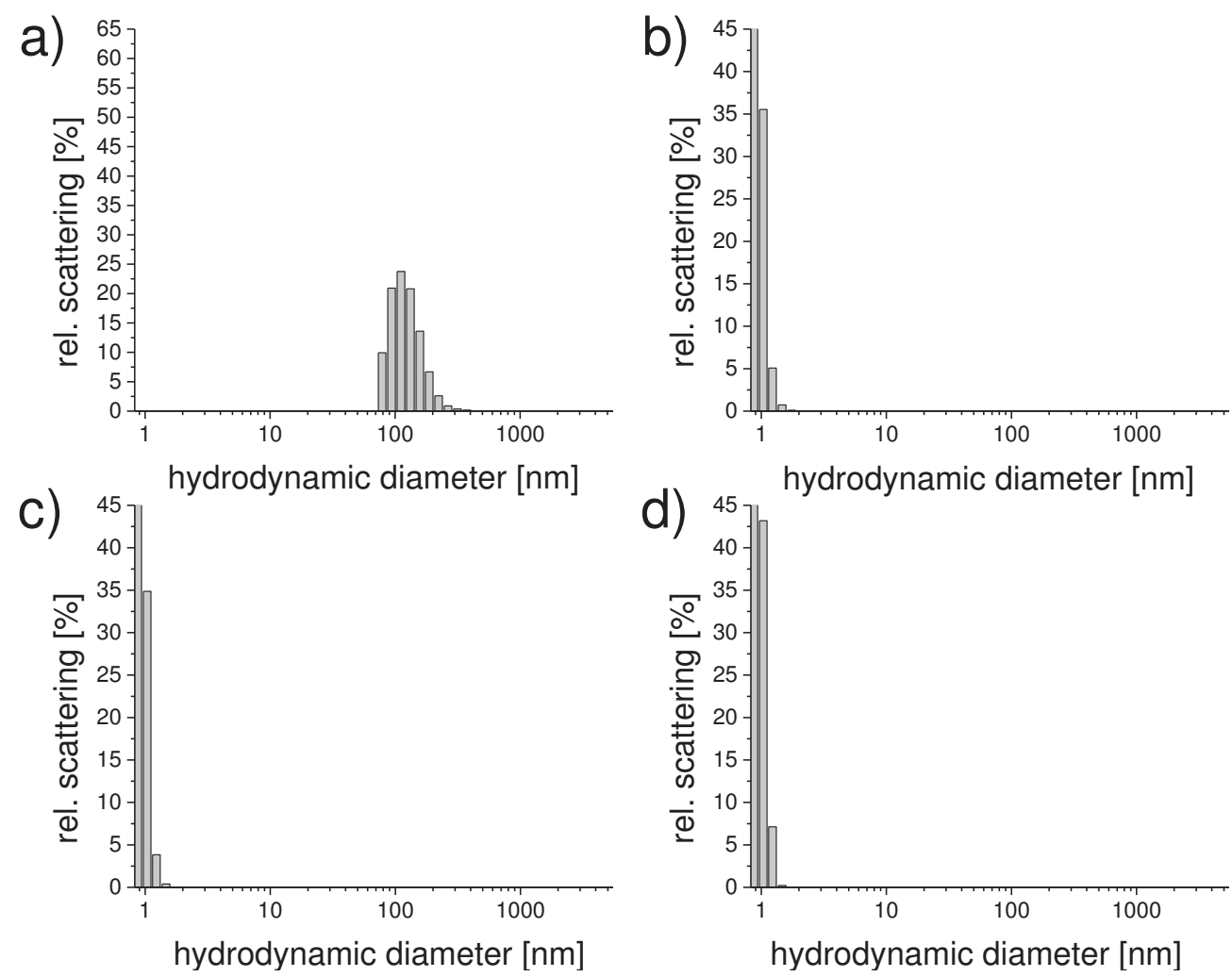

Figure 5.9: DLS analysis of SNPS prepared with 30\% Azo from Azo -PAMAM a) as assembled and b) after $\mathrm{Na}_{2} \mathrm{~S}_{2} \mathrm{O}_{4}$ addition, c) subsequently after $12 \mathrm{~h}$ UV light, d) subsequently after $8 \mathrm{~h}$ ambient light.

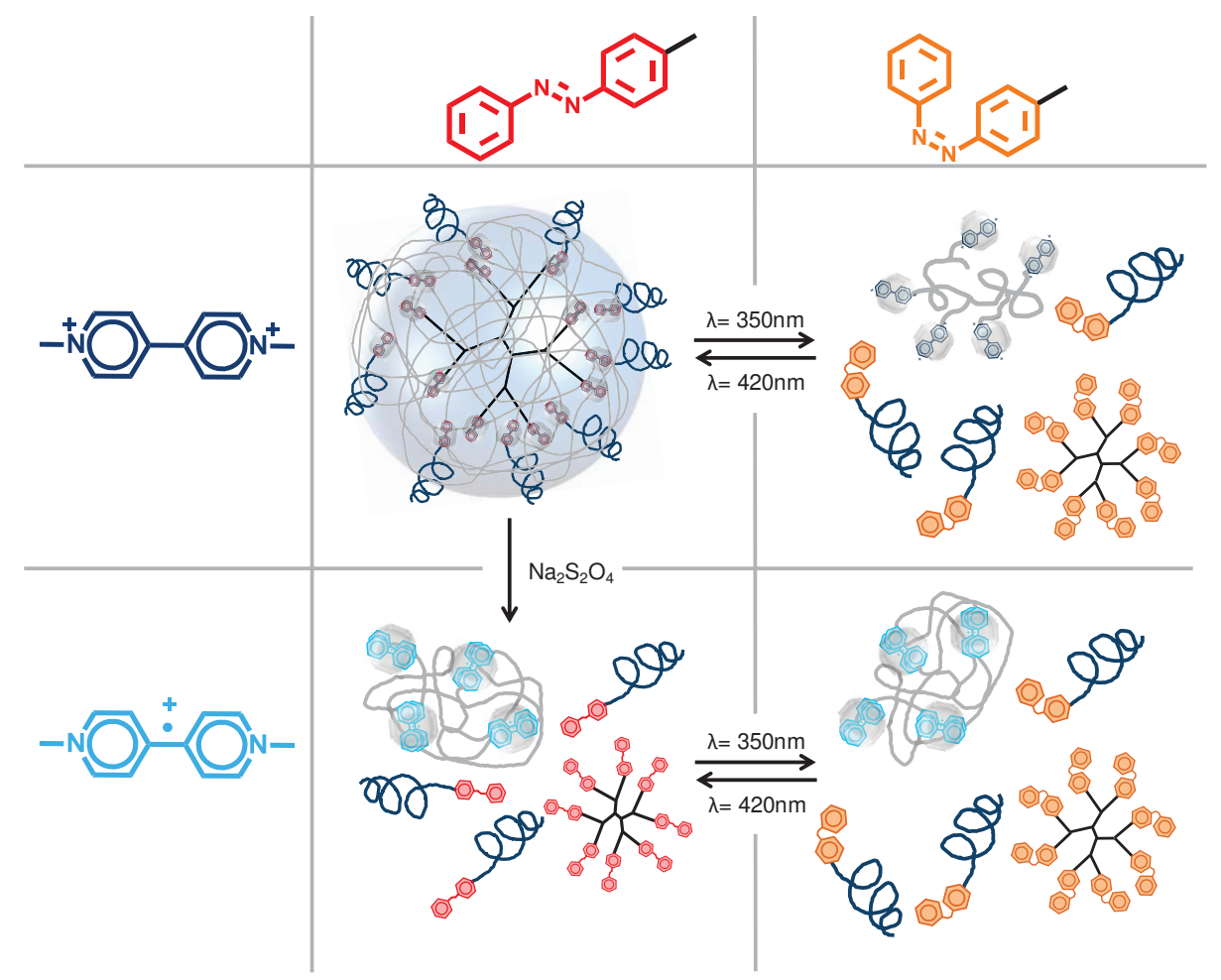

Figure 5.10 Molecular AND logic operator in dual-responsive SNPS: SNPS are only formed with transAzo and the MV dication; with Azo in the cis form (by photoirradiation) and/or MV in the radical cation form (by chemical reduction), only small building blocks are observed. 


\subsection{Conclusions}

In conclusion, we have developed a supramolecular strategy for the self-assembly of sizecontrolled supramolecular nanoparticles based on the ternary interaction between a MV polymer, mono- and multivalent Azo building blocks and $\mathrm{CB}[8]$. The heteroternary complex enables the particles to be assembled and disassembled, the latter triggered either by UV irradiation or by a chemical reductant. Reversible disassembly and reassembly can be induced by illumination by UV and Vis light, respectively, whereas irreversible disassembly occurs following chemical reduction. The dual responsive character as well as its reversibility make this SNP system a promising candidate for advanced materials engineering.

\subsection{Acknowledgements}

Jens Voskuhl is acknowledged for the synthesis of $\mathrm{AzO}_{8}$-PAMAM and fruitful discussions. Mark Smithers is acknowledged for taking the SEM images.

\subsection{Experimental Section}

\subsubsection{Materials}

Starting materials for organic synthesis were obtained from Sigma-Aldrich and used as received. MV-PEI was prepared as described in Chapter 3. Cucurbit[8]uril (CB[8]) was purchased from Strem Chemicals and its purity was assessed by microcalorimetric titration against paraquat. Deuterated solvents used for NMR spectroscopy were purchased from Cambridge Isotope and the water used for dialysis and SNP preparation was of MilliQ quality (Millipore, $R=18.2{\mathrm{M} \Omega \mathrm{cm}^{-1}}^{-1}$ ).

\subsubsection{Synthetic procedures}

\section{Methyl (E)-5-(4-(phenyldiazenyl)phenoxy)pentanoate}

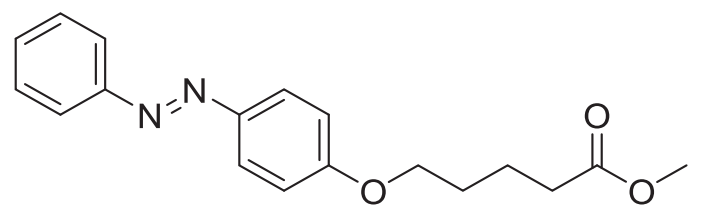

To a solution of methyl 5-bromopentanoate in acetonitrile ( $1 \mathrm{~g}, 5,1 \mathrm{mmol}, 1 \mathrm{eq}$ ) was added $\mathrm{K}_{2} \mathrm{CO}_{3}$ (6 g, $51 \mathrm{mmol}, 10 \mathrm{eq}$.) and the suspension was stirred for $30 \mathrm{~min}$ at $80^{\circ} \mathrm{C}$. Thereafter, phenylazobenzene was added $(1 \mathrm{~g}$, $5.1 \mathrm{mmol}, 1 \mathrm{eq}$.). Stirring was continued at $80^{\circ} \mathrm{C}$ for $18 \mathrm{~h}$, followed by removal of the solvent. The residue was dissolved in $30 \mathrm{~mL}$ of $\mathrm{CHCl}_{3}$ and extracted 3 times with distilled water $(20 \mathrm{~mL})$. The organic layer was separated and dried over $\mathrm{MgSO}_{4}$. The solvent was evaporated and the residue was subjected to silica gel column chromatography $\left(\mathrm{CHCl}_{3}\right)$. The product was obtained as bright orange solid. Yield: $1.31 \mathrm{~g}, 80 \% .{ }^{1} \mathrm{H}-\mathrm{NMR}(400 \mathrm{MHz}$, $\left.\mathrm{CDCl}_{3}\right): \delta=7.90-7.85(\mathrm{~m}, 4 \mathrm{H}, 4 \mathrm{CH}) ; 7.50-7.46(\mathrm{~m}, 2 \mathrm{H}, 2 \mathrm{CH}) ; 7.43-7.41(\mathrm{~m}, 1 \mathrm{H}, \mathrm{CH})$; 
6.99-6.97 (m , 2H, 2CH); 4.04-4.02 (m, $\left.2 \mathrm{H}, \mathrm{CH}_{2}\right) ; 3.67\left(\mathrm{~s}, 3 \mathrm{H}, \mathrm{CH}_{3}\right) ; 2.42-2.39(\mathrm{~m}, 2 \mathrm{H}$, $\left.\mathrm{CH}_{2}\right) ; 1.85-1.83\left(\mathrm{~m}, 4 \mathrm{H}, 2 \mathrm{CH}_{2}\right) .{ }^{13} \mathrm{C}-\mathrm{NMR}\left(100 \mathrm{MHz} \mathrm{CDCl}_{3}\right): \delta=174.04,161.77,152.83$, $147.06,130.59,129.25,125.08,122.76,114.91,67.94,51.80,33.85,28.80,21.81$. ESIMS (m/z): calculated for $\left[\mathrm{C}_{18} \mathrm{H}_{20} \mathrm{~N}_{2} \mathrm{O}_{3} \mathrm{H}\right]^{+}: 313.1$, found: 313.0 .

\section{(E)-5-(4-(Phenyldiazenyl)phenoxy)pentanoic acid}

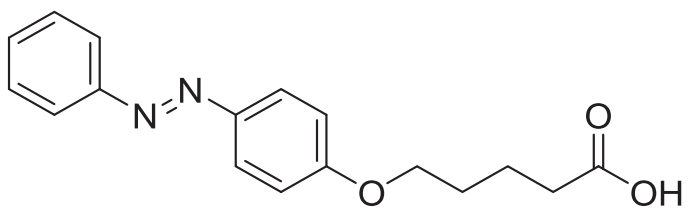

To a stirred solution of methyl (E)-5-(4(phenyldiazenyl)phenoxy)pentanoate $(1.30 \mathrm{~g}$, $4.1 \mathrm{mmol} 1$ eq.) in $30 \mathrm{~mL}$ of $\mathrm{MeOH} / \mathrm{THF}(1: 1)$ was added $\mathrm{LiOH}(2 \mathrm{~g}, 41 \mathrm{mmol}, 10$ eq.) dissolved in $15 \mathrm{~mL}$ distilled water. The reaction was stirred at room temperature for $3 \mathrm{~h}$. After that time the solution was acidified with $1 \mathrm{M}$ hydrochloric acid followed by dilution with $50 \mathrm{~mL}$ of $\mathrm{CHCl}_{3}$. The suspension was extracted 3 times with $30 \mathrm{~mL}$ of distilled water. The organic layer was dried over $\mathrm{MgSO}_{4}$ and the solvent was removed under reduced pressure. The product was obtained as brown-orange solid and was used without further purification. Yield: $1.15 \mathrm{~g}, 92 \% .{ }^{1} \mathrm{H}-\mathrm{NMR}\left(400 \mathrm{MHz} \mathrm{CDCl}_{3}\right): \delta=7.90-$ 7.85 (m, 4H, 4CH); 7.50-7.46 (m, 2H, 2CH); 7.44-7.41 (m, 1H, CH); 6.98-6.96 (m , 2H, $2 \mathrm{CH}) ; 4.06-4.04\left(\mathrm{~m}, 2 \mathrm{H}, \mathrm{CH}_{2}\right) ; 2,47-2,45\left(\mathrm{~m}, 2 \mathrm{H}, \mathrm{CH}_{2}\right) ; 1.87-1.85\left(\mathrm{~m}, 4 \mathrm{H}, 2 \mathrm{CH}_{2}\right) .{ }^{13} \mathrm{C}-$ NMR $\left(100 \mathrm{MHz}_{1} \mathrm{CDCl}_{3}\right): \delta=178.76,161.79,152.74,147.02,130.64,129.27,125.17$, 122.77, 114.93, 67.92, 33.63, 28.71, 21.58. ESI-MS (m/z): calculated for $\left[\mathrm{C}_{17} \mathrm{H}_{18} \mathrm{~N}_{2} \mathrm{O}_{3} \mathrm{H}\right]^{+}:$299.1, found: 299.0 .

\section{Perfluorophenyl (E)-5-(4-(phenyldiazenyl)phenoxy)pentanoate}

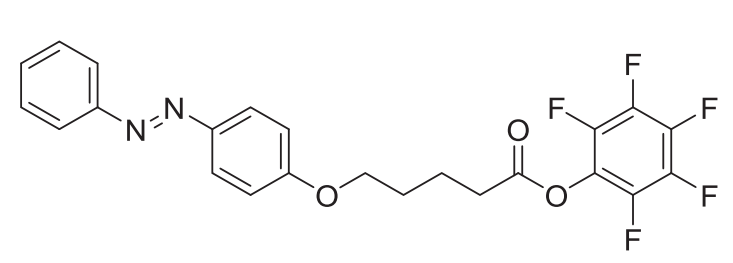

To a solution of (E)-5-(4-(phenyldiazenyl) phenoxy)pentanoic acid $(1 \mathrm{~g}, 3.35 \mathrm{mmol}, 1$ eq.) in $30 \mathrm{~mL}$ of THF was added dicyclohexylcarbodiimide $(1.1 \mathrm{~g}, 5 \mathrm{mmol}, 1.5$ eq.) and the mixture was stirred for $30 \mathrm{~min}$ at room temperature. After adding pentafluorophenol $(1 \mathrm{~g}, 5 \mathrm{mmol}, 1.5 \mathrm{eq}$.) stirring was continued for $18 \mathrm{~h}$. The suspension was filtered and the solvent was removed under reduced pressure. The residue was subjected to silica gel column chromatography $\left(\mathrm{CHCl}_{3}\right)$. After removal of the solvent the product was obtained as a bright orange solid. Yield: $1.2 \mathrm{~g}, 77 \% .{ }^{1} \mathrm{H}-\mathrm{NMR}\left(400 \mathrm{MHz}, \mathrm{CDCl}_{3}\right)$ : 7.92-7.85 (m, 4H, 4CH); 7.50-7.48 (m, $2 \mathrm{H}, 2 \mathrm{CH}) ; 7.44-7.40(\mathrm{~m}, 1 \mathrm{H}, \mathrm{CH}) ; 7.01-6.98(\mathrm{~m}, 2 \mathrm{H}, 2 \mathrm{CH}) ; 4.11-4.08(\mathrm{t}, \mathrm{J}=5.4 \mathrm{~Hz}, 2 \mathrm{H}$, $\left.\mathrm{CH}_{2}\right) ; 2,79-2,76\left(\mathrm{t}, \mathrm{J}=7.0 \mathrm{~Hz}, 2 \mathrm{H}, \mathrm{CH}_{2}\right) ; 2.01-1.95\left(\mathrm{~m}, 4 \mathrm{H}, 2 \mathrm{CH}_{2}\right) .{ }^{13} \mathrm{C}-\mathrm{NMR}(100 \mathrm{MHz}$, $\left.\mathrm{CDCl}_{3}\right): \delta=169.42,161.60,152.86,147.18,130.62,129.25,125.06,122.77,114.89$, 67.69, 33.15, 28.51, 21.77. ${ }^{19} \mathrm{~F}-\mathrm{NMR}\left(376 \mathrm{MHz}, \mathrm{CDCl}_{3}\right): \delta=-152.72$ (d, $J=18 \mathrm{~Hz}, 2 \mathrm{CF}$ ); $-157.94(\mathrm{t}, J=22 \mathrm{~Hz}, 2 \mathrm{CF}) ;-162.25(\mathrm{t}, J=22 \mathrm{~Hz}, 1 \mathrm{CF})$, ESI-MS (m/z): calculated for $\left[\mathrm{C}_{23} \mathrm{H}_{17} \mathrm{~F}_{5} \mathrm{~N}_{2} \mathrm{O}_{3} \mathrm{H}\right]^{+}: 465.1$, found: 465.0 . 


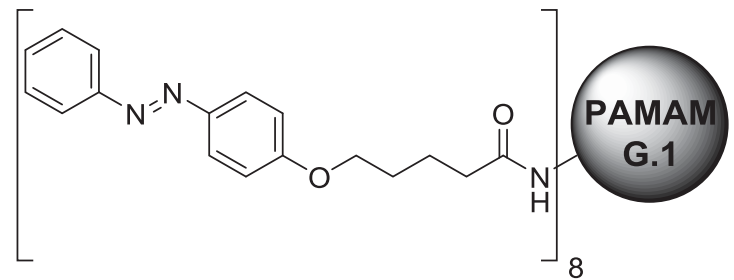

To a solution of perfluorophenyl (E)-5-(4(phenyldiazenyl)phenoxy)pentanoate (300 mg, $0.65 \mathrm{mmol}, 16$ eq.) in a 1:1 mixture of $10 \mathrm{~mL}$ of $\mathrm{CH}_{2} \mathrm{Cl}_{2}$ and DMF (1:1) was added PAMAM dendrimer generation 1 (50 mg, 0,04 mmol, 1 eq.) dissolved in 1 $\mathrm{mL}$ of DMF. The reaction was stirred under an atmosphere of argon for $18 \mathrm{~h}$. The resulting precipitate was filtered off and washed with $\mathrm{CH}_{2} \mathrm{Cl}_{2}, \mathrm{MeOH}$ and acetone. The residue was dried in vacuo yielding an orange solid. Yield: $33 \mathrm{mg}, 24 \%$. ${ }^{1} \mathrm{H}-\mathrm{NMR}$ (400 MHz, DMSO-d $\mathrm{d}_{6}$ ): 7.95 (bs, 8H, 8NH); 7.89 (bs, 8H, 8NH), 7.87-7.81 (m, 32H, $32 \mathrm{CH}$ ), 7.57-7.50 (m, 24H, $24 \mathrm{CH}) ; 7.10-7.05(\mathrm{~m}, 16 \mathrm{H}, 16 \mathrm{CH}) ; 6.81$ (bs, 4H, 4NH); 4.03 (t, $J=$ $\left.5.8 \mathrm{~Hz}, 16 \mathrm{H}, 8 \mathrm{CH}_{2}\right) ; 3.10-3.06\left(\mathrm{~m}, 40 \mathrm{H}, 20 \mathrm{CH}_{2}\right) ; 2.67-2.62\left(\mathrm{~m}, 24 \mathrm{H}, 12 \mathrm{CH}_{2}\right) ; 2.44-2.40$ $\left(\mathrm{m}, 12 \mathrm{H}, 6 \mathrm{CH}_{2}\right) ; 2.18-2.13\left(\mathrm{~m}, 40 \mathrm{H}, 20 \mathrm{CH}_{2}\right) ; 1.70-1.66\left(\mathrm{~m}, 32 \mathrm{H}, 16 \mathrm{CH}_{2}\right) ;$ MALDI-MS (m/z): calculated for $\left[\mathrm{C}_{198} \mathrm{H}_{256} \mathrm{~N}_{42} \mathrm{O}_{28} \mathrm{Na}\right]^{+}: 3695.5$ found: 3695.1 .

\section{Azobenzene-substituted poly(ethylene glycol) (Azo-PEG)}

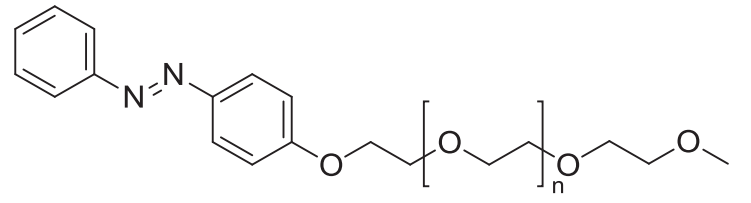

Polyethylene glycol monomethyl ether tosylate (average $\mathrm{M}_{\mathrm{W}} 5000 \mathrm{~g} / \mathrm{mol} ; 2 \mathrm{~g}, 0.4$ mmol), 4-hydroxyazobenzene (0.4 g, 2 $\mathrm{mmol})$ and $\mathrm{K}_{2} \mathrm{CO}_{3}(0.28 \mathrm{~g}, 2 \mathrm{mmol})$ were dissolved in $40 \mathrm{~mL}$ acetonitrile and stirred under reflux for 5 days. The solvent was evaporated and the residue redissolved in $20 \mathrm{~mL} \mathrm{CH} \mathrm{Cl}_{2}$. The excess of 4hydroxyazobenzene was removed by repeated precipitation in diethyl ether. The collected solid was dissolved in water and dialyzed against water for 5 days ( $M_{W}$ cut-off $2000 \mathrm{~g} / \mathrm{mol}$ ). The aqueous solution was freeze-dried to obtain a yellow product. Yield: $1.2 \mathrm{~g}, 60 \%,{ }^{1} \mathrm{H}-\mathrm{NMR}\left(600 \mathrm{MHz}, \mathrm{D}_{2} \mathrm{O}\right): 7.96(\mathrm{~d}, J=8.46 \mathrm{~Hz}, 2 \mathrm{H}, 2 \mathrm{CH}) ; 7.88(\mathrm{~d}, J=7.56$ $\mathrm{Hz}, 2 \mathrm{H}, 2 \mathrm{CH}) ; 7.65-7.61(\mathrm{~m}, 3 \mathrm{H}, 3 \mathrm{CH}) ; 7.25(\mathrm{~d}, J=7.25 \mathrm{~Hz}, 2 \mathrm{H}, 2 \mathrm{CH}) ; 3.9-3.4(\mathrm{~m}$, $417 \mathrm{H}, 417 \mathrm{CH}_{2}$ ) MALDI-MS (m/z): calculated for $\left[\mathrm{C}_{228} \mathrm{H}_{438} \mathrm{~N}_{2} \mathrm{O}_{107}\right]^{+}: 4919.8$ found: 4928.0.

\subsubsection{Methods}

\section{Supramolecular nanoparticle assembly}

For the preparation of the supramolecular nanoparticles, aqueous solutions of $\mathrm{CB}[8], \mathrm{MV}$ PEI and different concentrations of Azo-PEG were prepared before mixing. DMSO was used to dissolve different concentrations of $\mathrm{AzO}_{8}$-PAMAM. For example, for preparing a solution having $20 \%$ Azo moieties derived from Azo ${ }_{8}$-PAMAM, $1000 \mu \mathrm{L}$ of MV-PEI $(0.31$ 
$\mu \mathrm{M})$ was added to a previously prepared solution of $500 \mu \mathrm{L}$ Azo-PEG $(2.24 \mu \mathrm{M}), 10 \mu \mathrm{L}$

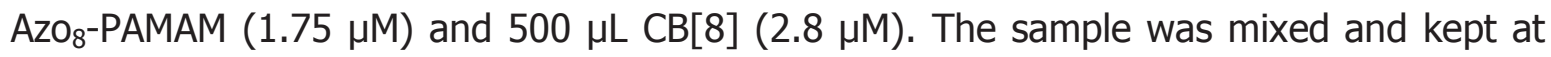
room temperature for 2 days before DLS and SEM analysis.

\section{Control experiments for supramolecular nanoparticle formation}

To evaluate whether SNP formation is triggered by the formation of the ternary complex between $\mathrm{CB}[8], \mathrm{MV}$ and $\mathrm{Azo}$, different control experiments were carried out. SNP formation using $20 \%$ Azo from $\mathrm{AzO}_{8}$-PAMAM was carried out in the absence of $\mathrm{CB}[8]$ or in the presence of $\mathrm{CB}[7]$ instead of $\mathrm{CB}[8]$. Hereto, solutions were prepared with $500 \mu \mathrm{L}$ water or $500 \mu \mathrm{L} \mathrm{CB}[7](2.8 \mu \mathrm{M})$ instead of $500 \mu \mathrm{L} \mathrm{CB}[8](2.8 \mu \mathrm{M})$.

\section{Light response of supramolecular nanoparticles}

To evaluate the light responsiveness of the particles, SNPs containing 30\% Azo from $\mathrm{Azo}_{8}$-PAMAM, were prepared. After analysis by DLS, the samples were irradiated with UV light $(\lambda<400 \mathrm{~nm})$ for different times. DLS analysis was performed immediately after exposure, while keeping the SNP samples in the dark. It has to be noted that the obtained hydrodynamic diameters of the UV-treated SNP samples (shown in Figure 5.5) are not absolute values since the polydispersity of the samples was too high.

Reversibility of SNP disassembly and re-assembly was evaluated by first formation and characterization of SNP with $30 \%$ Azo from Azo - PAMAM. Subsequently, the samples were irradiated overnight with UV light $(\lambda \leq 400 \mathrm{~nm})$ followed by DLS to prove particle disassembly. SNP re-assembly was induced by keeping the samples for 8-10 $\mathrm{h}$ under ambient light conditions. Particle reformation was evaluated by DLS. This procedure was repeated 4 times with the same particle solution.

\section{Reductive supramolecular nanoparticle disassembly}

To evaluate whether reduction of the MV species leads to disassembly of the SNPs, particles were prepared and analyzed by DLS. SNP disassembly was carried out by adding $10 \mu \mathrm{L}$ aqueous $\mathrm{Na}_{2} \mathrm{~S}_{2} \mathrm{O}_{4}(5 \mathrm{mM})$ to the SNP sample under inert conditions. Particle size analysis was carried out by DLS after $8 \mathrm{~h}$.

Combination of reductive and UV-triggered supramolecular nanoparticle disassembly SNPs $\mathrm{h}$ were prepared and analyzed by DLS. Thereafter, $10 \mu \mathrm{L}$ aqueous $\mathrm{Na}_{2} \mathrm{~S}_{2} \mathrm{O}_{4}(5 \mathrm{mM})$ was added to the SNP sample under nitrogen. Particle size analysis was carried out by DLS after $8 \mathrm{~h}$ and after subsequent UV irradiation for $12 \mathrm{~h}$. The reassembly ability of the reduced and UV-treated SNPs was tested after $8 \mathrm{~h}$ under ambient light conditions. 


\subsubsection{Equipment}

\section{DLS analysis (DLS)}

Size tunability of the SNPs was assessed using a Malvern Zetasizer at $25^{\circ} \mathrm{C}$. SNP disassembly and re-assembly experiments were performed with a Nanotrac wave from Anaspec operating with the Microtrac FLEX Operating Software. The observed sizes and standard derivations of the supramolecular NPs are based on the average number distributions of minimum five individual measurements per sample. Three samples were measured for each reported NP formulation. In total, particles with sizes ranging from 0.8 to $6400 \mathrm{~nm}$ were detected. The DLS curves shown in this chapter are representative for the individual measurements.

\section{Scanning electron microscopy (SEM)}

All SEM images were taken with a Carl Zeiss Merlin scanning electron microscope. Aqueous SNP solutions were drop-cast on Formvar coated copper TEM grids. The solutions were dried and analyzed without further treatment of the samples.

\section{UV light irradiation}

UV light irradiation experiments were carried out with a Hönle bluepoint 2 easycure using a shortpass filter $\lambda \leq 400 \mathrm{~nm}$. After formation, the SNPs were treated with UV light for different periods. Therefore the samples were filled in a quartz cuvette and exposed to light with $\lambda \leq 400 \mathrm{~nm}$. To analyze the ongoing disassembly process, the samples were analyzed by DLS in dark and UV-irradiation was continued.

\section{$\underline{\text { UV/Vis spectroscopy }}$}

UV/Vis absorption spectra were recorded using a Perkin Elmer Lambda 850 UV-Vis spectrometer. To investigate the photoisomerization behavior of azobenzene in the heteroternary complex at room temperature, an aqueous solution of $\mathrm{CB}[8]$, paraquat and Azo-PEG (25 $\mu \mathrm{M}, 25 \mu \mathrm{M}$, and $23.75 \mu \mathrm{M}$, respectively) was prepared and measured before and after UV irradiation. Furthermore, the time dependent cis-trans isomerization was controlled by measuring the UV absorbance after $1 \mathrm{~min}, 2 \mathrm{~min}, 5 \mathrm{~min}, 10 \mathrm{~min}, 15 \mathrm{~min}, 20$ min and 30 min while keeping the same solution at ambient light.

\section{NMR spectroscopy}

${ }^{1} \mathrm{H}$ and ${ }^{13} \mathrm{C}$ NMR spectra were recorded on Bruker $400 \mathrm{MHz}$ and $600 \mathrm{MHz}$ spectrometers. ${ }^{1} \mathrm{H}$ and ${ }^{13} \mathrm{C}$ chemical shift values are reported as $\delta$ (ppm) using the residual solvent signal as internal standard. In addition, the ratio of trans and cis isomers of Azo in Azo-PEG before and after UV irradiation in $\mathrm{D}_{2} \mathrm{O}$ was determined using ${ }^{1} \mathrm{H}-\mathrm{NMR}$. 


\section{Mass spectrometry}

Mass analysis was done using the matrix-assisted laser desorption ionization (MALDI) and electrospray ionization using a Voyager DE-RP and a micromass LCT from Waters/Micromass, respectively.

\subsection{References}

1. M. E. Davis, J. E. Zuckerman, C. H. J. Choi, D. Seligson, A. Tolcher, C. A. Alabi, Y. Yen, J. D. Heidel and A. Ribas, Nature, 2010, 464, 1067-1070.

2. H. Wang, S. Wang, H. Su, K.-J. Chen, A. L. Armijo, W.-Y. Lin, Y. Wang, J. Sun, K.-i. Kamei, J. Czernin, C. G. Radu and H.-R. Tseng, Angewandte Chemie International Edition, 2009, 48, 4344-4348.

3. L. Y. Liu R, He B, Li Y, Wang G, Chang S, Gu Z., International Journal of Nanomedicine, 2012, 7, 5249-5258.

4. T. Kakuta, Y. Takashima, M. Nakahata, M. Otsubo, H. Yamaguchi and A. Harada, Advanced Materials, 2013, 25, 2849-2853.

5. $\quad$ K. L. Liu, Z. Zhang and J. Li, Soft Matter, 2011, 7, 11290-11297.

6. E. A. Appel, X. J. Loh, S. T. Jones, F. Biedermann, C. A. Dreiss and O. A. Scherman, Journal of the American Chemical Society, 2012, 134, 11767-11773.

7. G. Gröger, W. Meyer-Zaika, C. Böttcher, F. Gröhn, C. Ruthard and C. Schmuck, Journal of the American Chemical Society, 2011, 133, 8961-8971.

8. P. A. Korevaar, S. J. George, A. J. Markvoort, M. M. J. Smulders, P. A. J. Hilbers, A. P. H. J. Schenning, T. F. A. De Greef and E. W. Meijer, Nature, 2012, 481, 492-496.

9. Y. Liu, Z. Huang, X. Tan, Z. Wang and X. Zhang, Chemical Communications, 2013, 49, 5766-5768.

10. J. Voskuhl, M. Waller, S. Bandaru, B. A. Tkachenko, C. Fregonese, B. Wibbeling, P. R. Schreiner and B. J. Ravoo, Organic \& Biomolecular Chemistry, 2012, 10, 4524-4530.

11. M. V. Rekharsky and Y. Inoue, Chemical Reviews, 1998, 98, 1875-1918.

12. J. Lagona, P. Mukhopadhyay, S. Chakrabarti and L. Isaacs, Angewandte Chemie International Edition, 2005, 44, 4844-4870.

13. A. González-Campo, M. Brasch, D. A. Uhlenheuer, A. Gómez-Casado, L. Yang, L. Brunsveld, J. Huskens and P. Jonkheijm, Langmuir, 2012, 28, 16364-16371.

14. Q. An, J. Brinkmann, J. Huskens, S. Krabbenborg, J. de Boer and P. Jonkheijm, Angewandte Chemie International Edition, 2012, 51, 12233-12237.

15. Q. Hu, W. Li, X. Hu, Q. Hu, J. Shen, X. Jin, J. Zhou, G. Tang and P. K. Chu, Biomaterials, 2012, 33, 6580-6591.

16. H. Izawa, K. Kawakami, M. Sumita, Y. Tateyama, J. P. Hill and K. Ariga, Journal of Materials Chemistry B, 2013, 1, 2155-2161.

17. A. Kulkarni, W. Deng, S.-h. Hyun and D. H. Thompson, Bioconjugate Chemistry, 2012, 23, 933-940.

18. Y. Ping, Q. Hu, G. Tang and J. Li, Biomaterials, 2013, 34, 6482-6494.

19. J.-H. Lee, K.-J. Chen, S.-H. Noh, M. A. Garcia, H. Wang, W.-Y. Lin, H. Jeong, B. J. Kong, D. B. Stout, J. Cheon and H.-R. Tseng, Angewandte Chemie International Edition, 2013, $52,4384-4388$. 
20. S. K. M. Nalluri, J. Voskuhl, J. B. Bultema, E. J. Boekema and B. J. Ravoo, Angewandte Chemie International Edition, 2011, 50, 9747-9751.

21. S. Angelos, Y.-W. Yang, N. M. Khashab, J. F. Stoddart and J. I. Zink, Journal of the American Chemical Society, 2009, 131, 11344-11346.

22. H. Yan, C. Teh, S. Sreejith, L. Zhu, A. Kwok, W. Fang, X. Ma, K. T. Nguyen, V. Korzh and Y. Zhao, Angewandte Chemie International Edition, 2012, 51, 8373-8377.

23. K. Peng, I. Tomatsu and A. Kros, Chemical Communications, 2010, 46, 4094-4096.

24. J. Wu and L. Isaacs, Chemistry - A European Journal, 2009, 15, 11675-11680.

25. F. Tian, D. Jiao, F. Biedermann and O. A. Scherman, Nature Communications, 2012, 3, 1207.

26. J. del Barrio, P. N. Horton, D. Lairez, G. O. Lloyd, C. Toprakcioglu and O. A. Scherman, Journal of the American Chemical Society, 2013, 135, 11760-11763.

27. W. S. Jeon, H.-J. Kim, C. Lee and K. Kim, Chemical Communications, 2002, 1828-1829.

28. U. Pischel, J. Andréasson, D. Gust and V. F. Pais, ChemPhysChem, 2013, 14, 28-46. 


\section{Chapter 6}

\section{Programmed Disassembly of Supramolecular Nanoparticles Stabilized by Heteroternary CB[8] Host- Guest Interactions}

Controlled release is an important component for the in vivo performance of drug delivering nanoparticles (NPs). Therefore, the control over and understanding of the release mechanism, e.g. by disassembly or degradation of the carrier, is essential for the optimization of NP formulations. This chapter presents a supramolecular toolbox approach for the formation and UV-induced disassembly of supramolecular nanoparticles (SNPS) which are either exclusively stabilized by cucurbit[8]uril (CB[8])/methyl viologen (MV)/azobenzene (Azo) interactions or $\mathrm{CB}[8] / \mathrm{MV} /$ naphthol (Np) interactions, or by a combination of both. Photoisomerization of the Azo units enables UV-triggered disassembly of the $\mathrm{CB}[8] / \mathrm{MV} / \mathrm{Azo}$ host-guest complex. Depending on the valency of the electron-rich guest moieties (Np or Azo), either SNPs with a UV-responsive shell or a UVresponsive core were formed by assembling SNPs using a mixture of Azo and Np bearing guest molecules. In contrast, non-responsive SNPs or SNPs which disintegrate at both the core and the shell were formed by using exclusively $\mathrm{CB}[8] / \mathrm{MV} / \mathrm{Np}$ or $\mathrm{CB}[8] / \mathrm{MV} / \mathrm{Azo}$ interactions, respectively. 


\subsection{Introduction}

Inorganic and organic nanoparticles (NPs) of different compositions are versatile platforms for biomedical applications because they are able to carry small molecules or act as diagnostic tools. ${ }^{1-7}$ Above all, selectively triggered nanoparticle (NP) disassembly is of substantial interest as encapsulated drugs or imaging agents can be released upon NP degradation. ${ }^{8-10}$ In particular, in drug delivery applications, control over the rate of degradation is of high importance to attain a constant and prolonged drug dose in the blood flow. When NP degradation occurs too fast, this can lead to a peak in drug concentration followed by a fast drop-off until re-administration, which is known to minimize the therapeutic performance of drug delivery vectors. Therefore, biodegradable polymer NPs and mesoporous silica nanoparticles have been developed to achieve prolonged drug release. ${ }^{11,12}$

In oncology, most imaging and drug delivery NPs take advantage of the enhanced permeability and retention (EPR) effect, i.e. a preferential uptake of the NPs by the tumor caused by the higher permeability and porosity of tumor tissue in comparison to normal tissue. ${ }^{13}$ This passive targeting enables NPs with sizes below $100 \mathrm{~nm}$ to penetrate and release the active compounds in the desired tissue. For instance, Besenbacher and coworkers have shown that especially small NPs can re-enter the bloodstream after penetration. ${ }^{14}$ Accumulation of NPs by aggregation is an alternative method to avoid outdiffusion of NPs from the carcinogenic tissue. Different strategies to induce NP aggregation have been reported, however mostly restricted to inorganic NPs. ${ }^{15-18}$ Examples of triggered NP disassembly or degradation employ external stimuli such as $\mathrm{pH}_{1}{ }^{19,20}$ temperature, ${ }^{21}$ chemical reduction, ${ }^{22}$ light $^{23}$ or combinations thereof. ${ }^{24,25}$ Despite the interest in finding ways to control the degradation of NPs, or to trigger the release of small molecules from the NPs, very few studies elucidate the underlying decomposition mechanism in more detail. ${ }^{26,27}$

Supramolecular nanoparticles (SNPs) established by multiple host-guest interactions form a specific category of responsive NPs. ${ }^{28-30}$ Their intrinsically stable yet reversible character and their chemical flexibility has been used to encapsulate DNA, ${ }^{31,32}$ drugs $^{33-35}$ and imaging agents ${ }^{36,37}$ for the formation of biomedically relevant nanovectors. In the previous chapters, SNP formation was achieved by employing multivalent heteroternary host-guest interactions between cucurbit[8]uril (CB[8]), methyl viologen (MV) and either naphthol (Np) or trans-azobenzene (Azo) as the second, electron-rich guest molecule. Hereto, MV was grafted onto poly(ethylene imine) (PEI) while the multivalent poly(amido amine) (PAMAM) dendrimer and monovalent poly(ethylene glycol) (PEG) were both functionalized with $\mathrm{Np}$ or Azo. Irrespective of the stabilizing heteroternary complex, the electron-rich guest-bearing PAMAM dendrimers reside in the SNP core by multivalent interactions with MV-PEI and $\mathrm{CB}[8]$, whereas the monovalent guest-functionalized PEG 
assembles at the exterior to ensure colloidal stability. The photoresponsive and reversible trans-cis isomerization of Azo has led to the selective disassembly of the heteroternary complex, ${ }^{38}$ causing reversible SNP disassembly for the Azo-based SNPs. Interestingly, the disintegration of the Azo-based SNPs occurred via a transient aggregated state caused by the different valencies of the Azo-bearing components and the incomplete photoswitching of Azo (see Chapter 5).

In this chapter, we present a toolbox approach for the fabrication of SNPs, in which the UV-induced particle disassembly can be regulated by introducing photo-responsive Azobearing components selectively either in the SNP shell or in the core. This enables the formation of SNPs for which the UV-triggered disassembly can be pre-programmed by the composition of the SNPs.

\subsection{Results and Discussion}

\subsubsection{SNP toolbox approach for programmable particle disassembly}

As illustrated in Scheme 6.1, MV-PEI and $\mathrm{CB}[8]$ were assembled into SNPs by heteroternary complexes formed with either $\mathrm{Np}_{8}$-PAMAM or $\mathrm{AzO}_{8}$-PAMAM as multivalent core-forming crosslinker and with Np-PEG or Azo-PEG as monovalent stopper assembling in the SNP shell. SNPs were formed by solely $\mathrm{CB}[8] / \mathrm{MV} / \mathrm{Np}$ or $\mathrm{CB}[8] / \mathrm{MV} / \mathrm{Azo}$ complexes as reported in Chapters 3 and 5 or by a combination of both heteroternary complexes. As the binding constants of the two heteroternary complexes are similar ${ }^{38,39}$ and the $\mathrm{Np}$ and Azo guest moieties are similar in size, no difference in SNP formation and resulting SNP size is expected.

The placement of the responsive Azo moiety in shell, core, or both, enables the formation of SNPs which undergo different responses upon treatment with UV light. As shown in Scheme 6.1 , the SNPs stabilized exclusively by CB[8]/MV/Np interactions should not undergo any change upon UV irradiation, while the SNPs formed by $\mathrm{CB}[8] / \mathrm{MV} / \mathrm{Azo}$ interactions in both shell and core show a time-differentiated response of the core and shell (see Chapter 5). As studied here, SNPs in which the core is assembled from $\mathrm{CB}[8] / \mathrm{MV} / \mathrm{Np}$ interactions and the shell from $\mathrm{CB}[8] / \mathrm{MV} / \mathrm{Azo}$ interactions are expected to aggregate by multiple $\mathrm{CB}[8] / \mathrm{MV} / \mathrm{Np}$ interactions in the core upon photoswitching of the SNP shell. Conversely, SNPs with Azo in the core and Np in the shell should shrink by the loss of the Azo building blocks from the SNP core upon photoswitching. 
a)

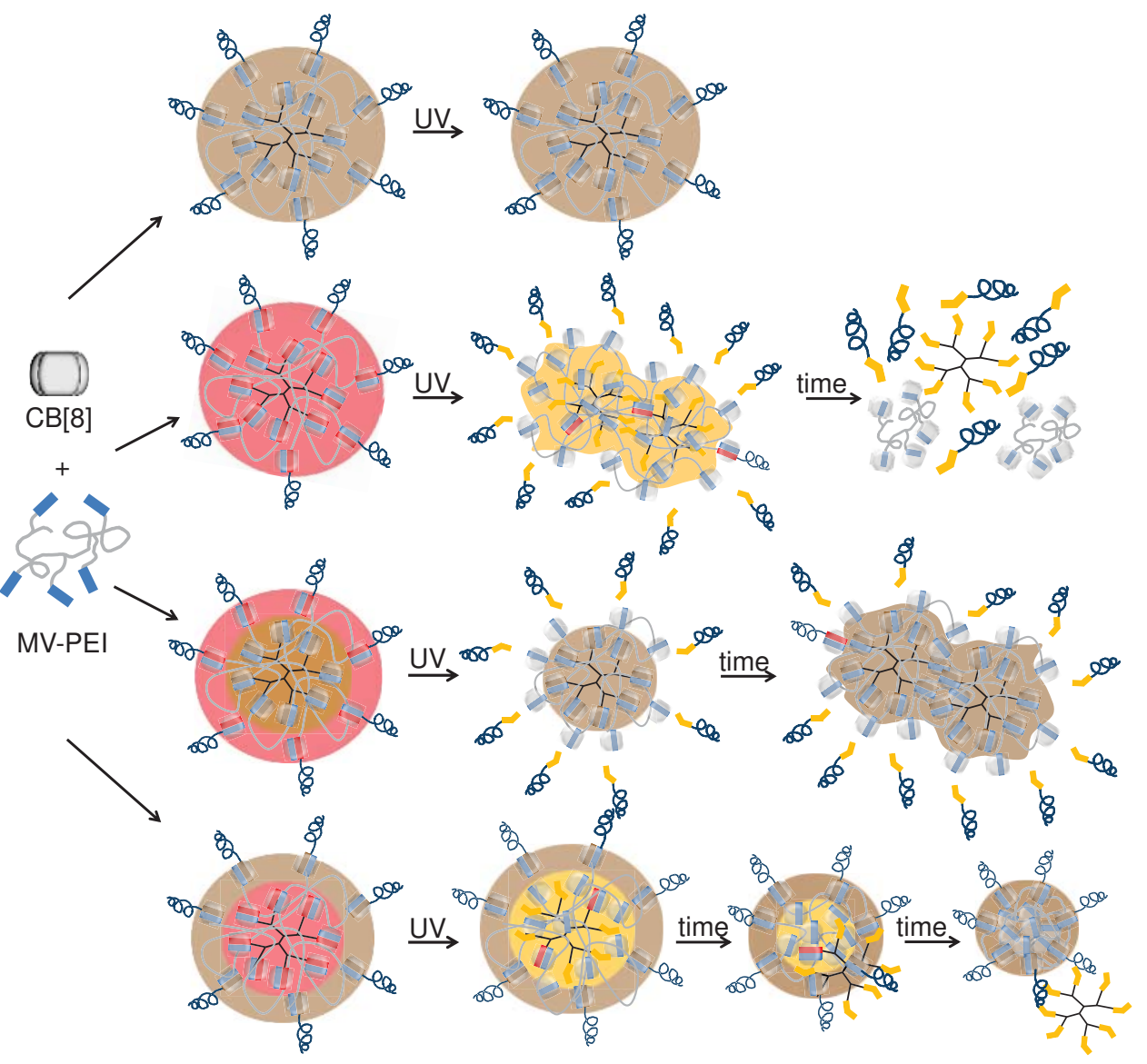

b)

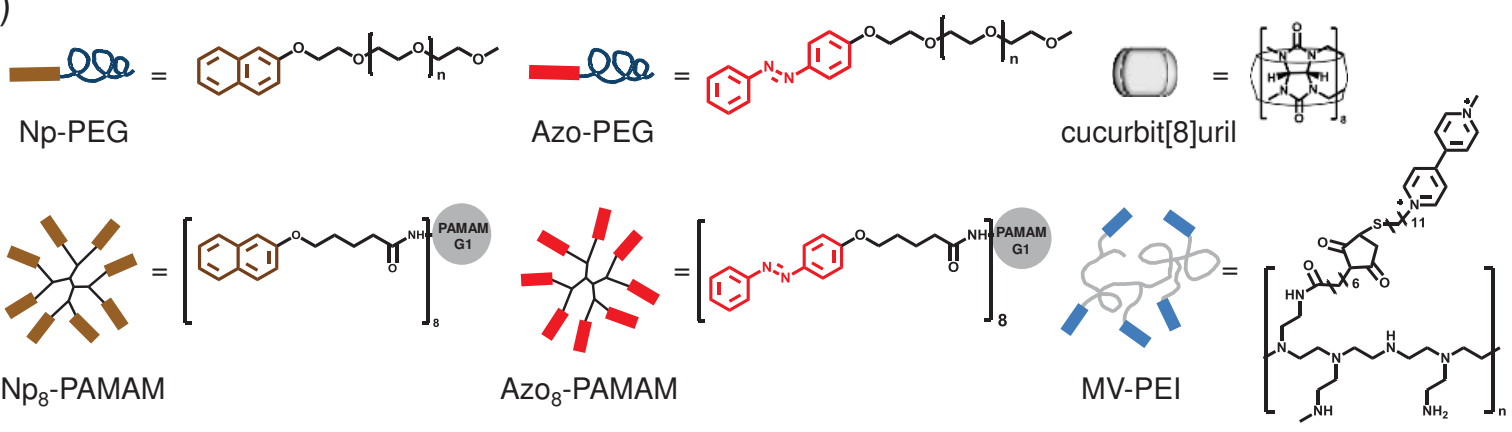

c)

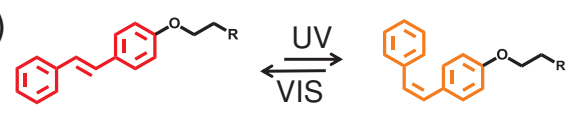

d)

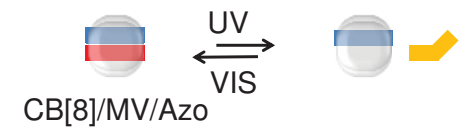

Scheme 6.1: a) Schematic representation of the assembly of supramolecular nanoparticles (SNPS) mediated by the heteroternary complexes between cucurbit[8]uril (CB[8]), methyl viologen (MV), and either naphthol (Np) or azobenzene (Azo), and the UV light-induced disassembly mechanisms of the different SNPS. b) The supramolecular building blocks involved in SNP assembly: Azo-PEG, Azo ${ }_{8}-P A M A M, N p-P E G, N p_{8}-P A M A M, M V-P E I$ and CB[8]. c) Photoisomerization of Azo moieties. d) Schematic representation of the UV-triggered, reversible dissociation of the heteroternary complex between $C B[8], M V$ and Azo. 


\subsubsection{SNPs with a photoswitchable shell}

Based on the concept described above, SNPs were formed using $C B[8] / \mathrm{MV} / \mathrm{Np}$ interactions in the core and $\mathrm{CB}[8] / \mathrm{MV} / \mathrm{Azo}$ interactions in the shell. Therefore, the multivalent dendrimer $\mathrm{Np}_{8}$-PAMAM and monovalent Azo-PEG were used for SNP preparation, in the presence of MV-PEI and $\mathrm{CB}[8]$. To ensure comparability, all SNP formulations reported here were prepared using equimolar concentrations $(0.7 \mu \mathrm{M})$ of $\mathrm{CB}[8], \mathrm{MV}$, and the second guest molecule (total amount of Azo and/or Np), with $30 \%$ of the latter derived from the multivalent core-forming dendrimer (and $70 \%$ of the monovalent guest-PEG). After mixing the supramolecular building blocks, the SNP samples were kept for 2 days at RT and analyzed by scanning electron microscopy (SEM) and dynamic light scattering (DLS) prior to SNP disassembly via UV light.

SNPs with sizes of $129 \pm 5 \mathrm{~nm}$ (DLS) were formed by self-assembly of $\mathrm{Np}_{8}$-PAMAM, AzoPEG, MV-PEI and CB[8] (Figure 6.1). This size compares well with sizes observed before for the systems using only Np (126 $\pm 16 \mathrm{~nm}$, see Chapter 3$)$ and only Azo (110 $\pm 19 \mathrm{~nm}$, see Chapter 5). Upon irradiation with UV light, immediate aggregation (starting after 10 min) was observed by DLS, continuing for 3-4 h until only a peak at $1 \mathrm{~nm}$ persisted. This peak at $1 \mathrm{~nm}$ is an artifact indicating the presence of insufficient material dispersed in solution. These results indicate the UV-induced, uncontrolled aggregation of the supramolecular building blocks, and subsequent sedimentation of the polymeric materials. Remarkably, after keeping the SNP samples for $24 \mathrm{~h}$ at ambient light, the reformation of SNPs with a size of $122 \pm 20 \mathrm{~nm}$ was observed by DLS. It should be noted that the aggregation behavior induced by UV switching observed here is different from the transient aggregation observed for the Azo-Azo system (Chapter 5). In the present system, the particles were found to increase in size monotonously, finally leading to invisibility by DLS, whereas in the previously studied Azo-Azo system, particles where found to get smaller after initial aggregation, finally leading to completely dissolved and dissociated building blocks. 


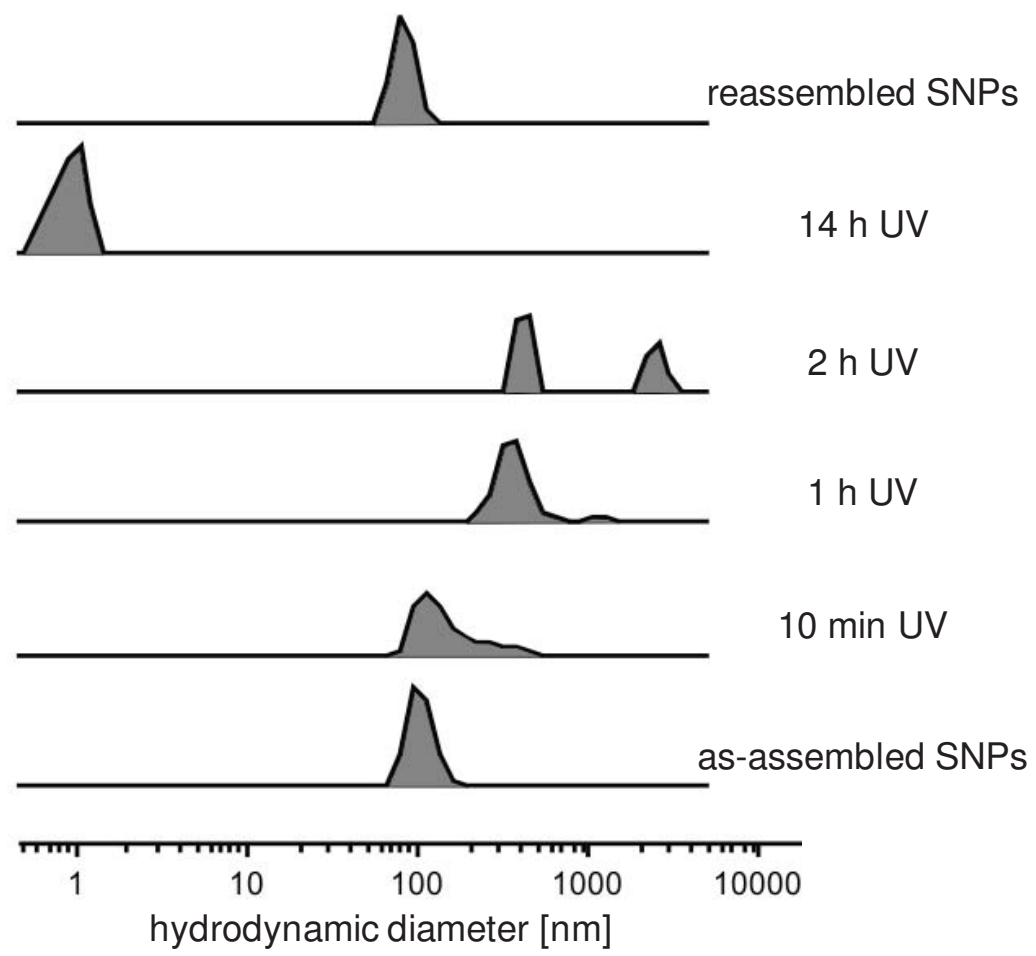

Figure 6.1: DLS size determination of as-assembled SNPS (prepared from Azo-PEG, Np-PAMAM $(A z o: N p=70: 30), M V-P E I$ and $C B[8]$ with $C B[8]: M V:(A z O+N p)=1: 1: 1)$, after UV irradiation for different amounts of time, and subsequently after $24 \mathrm{~h}$ ambient light.

SEM images confirm the observations made by DLS for the SNP samples prepared from Azo-PEG, Np 8 -PAMAM, MV-PEI and CB[8]. Clear spherical SNPs (73 $\pm 11 \mathrm{~nm}$ ) were visible after self-assembly, whereas only aggregated polymeric material was detected after UV treatment (12 h). Similar to DLS, SEM shows SNP reformation with a size of $76 \pm 23 \mathrm{~nm}$ after keeping the aggregated sample for $24 \mathrm{~h}$ at ambient light.
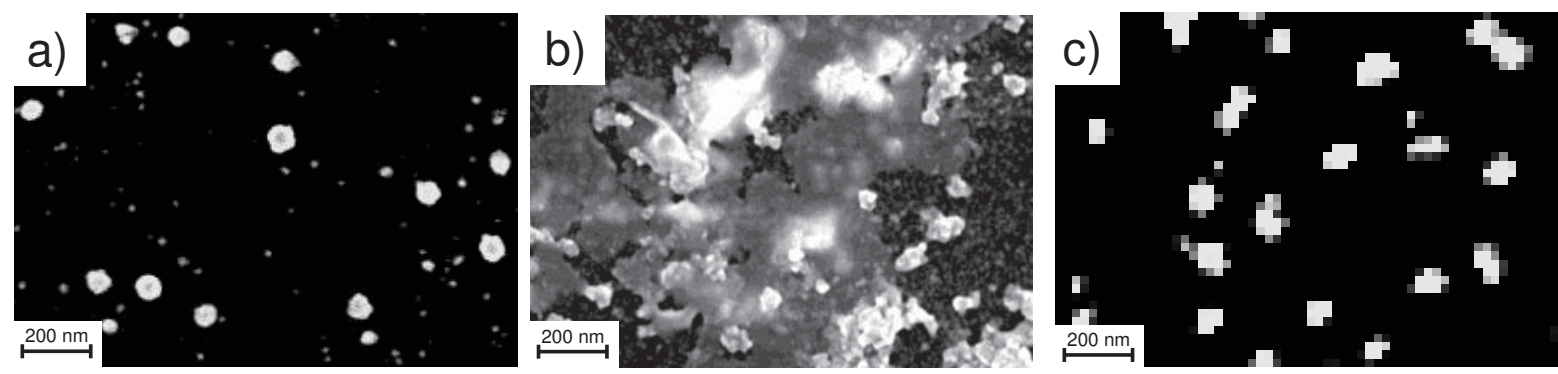

Figure 6.2: SEM images of SNPS (prepared from Azo-PEG, Np - PAMAM (Azo:Np = 70:30), MV-PEI and $C B[8]$ with $C B[8]: M V:(A z O+N p)=1: 1: 1)$ a) after self-assembly, b) after $14 \mathrm{~h}$ UV light, and c) subsequently after $24 \mathrm{~h}$ ambient light.

SEM images observed for SNPs formed exclusively from $\mathrm{CB}[8] / \mathrm{MV} / \mathrm{Azo}$ interactions showed initial aggregation after UV treatment $(3 \mathrm{~h}$ ) but complete SNP disintegration after further $8 \mathrm{~h}$ of UV irradiation (Figure 6.3 ). These results are in accordance with the DLS data presented in Chapter 5, and underline the difference in aggregation behavior 
between these systems as described above. By comparing the extent of aggregation observed for UV-treated SNPs stabilized by $\mathrm{CB}[8] / \mathrm{MV} / \mathrm{Azo}$ interactions in the shell and $\mathrm{CB}[8] / \mathrm{MV} / \mathrm{Np}$ in the core (Figure $6.2 \mathrm{~b}$ ) with UV-treated SNPs stabilized by solely $\mathrm{CB}[8] / \mathrm{MV} /$ Azo interactions (Figure $6.3 \mathrm{a}$ and $\mathrm{b}$ ), more clustering is evident for the SNPS with $\mathrm{Np}$ in the core. This observation is in agreement with the differences in disassembly mechanism. Shell disruption leads to uncontrolled aggregation in both types of SNPs, but the amount of crosslinks is lower in the solely $\mathrm{CB}[8] / \mathrm{MV} / \mathrm{Azo}$ stabilized SNPs as UV irradiation partially disrupts the multivalent interactions present in the SNP core immediately after short UV irradiation. Furthermore, UV treatment for $12 \mathrm{~h}$ led to complete SNP disintegration in these SNPs as visible in Figure $6.3 \mathrm{~b}$, which did not occur for the aggregated CB[8]/MV/Np-SNPs.
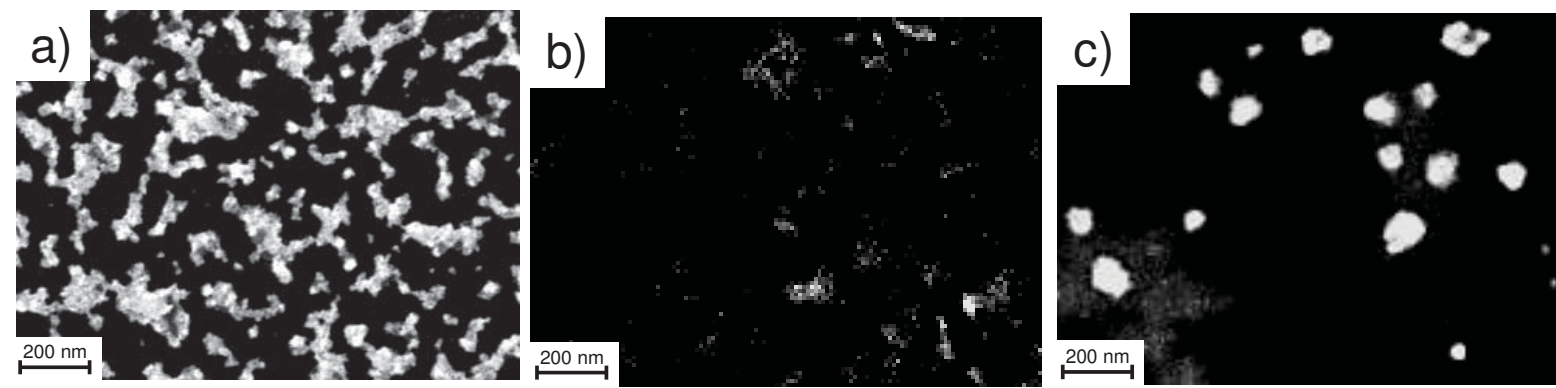

Figure 6.3: SEM images of SNPS (prepared from Azo-PEG, Azos-PAMAM (30\% Azo from Azo $8^{-}$ $P A M A M), M V-P E I$ and $C B[8]$ with CB[8]:MV:Azo = 1:1:1) a) after $3 \mathrm{~h}$ UV light, b) after an additional $8 \mathrm{~h}$ UV light treatment, and c) after subsequent $24 \mathrm{~h}$ at ambient light.

To evaluate whether SNPs stabilized by solely $\mathrm{CB}[8] / \mathrm{MV} / \mathrm{Np}$ interactions degrade upon UV irradiation, SNPs were formed by the self-assembly of $\mathrm{Np}_{8}$-PAMAM, Np-PEG, MV-PEI and $\mathrm{CB}[8]$ and treated with UV light for $14 \mathrm{~h}$ followed by visible light for $24 \mathrm{~h}$. As shown in Figure 6.4 the SNP size by DLS did not vary significantly before (112 $\pm 25 \mathrm{~nm}$ ) and after 14 h UV irradiation (134 $\pm 23 \mathrm{~nm}$ ), nor after subsequent visible light treatment (117 \pm 15 $\mathrm{nm})$.

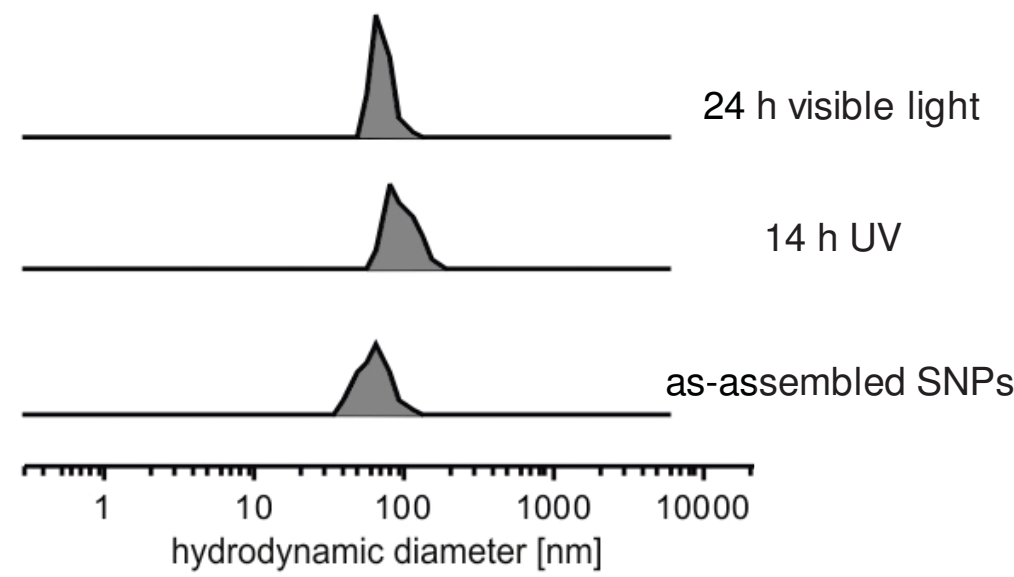

Figure 6.4: DLS size determinations of SNPs (prepared from Np-PEG, $N p_{8}-P A M A M$ (30\% Np from $\left.N p_{8}-P A M A M\right), M V-P E I$ and $C B[8]$ with CB[8]:MV:Np = 1:1:1) after self-assembly, after $14 \mathrm{~h} U \mathrm{~V}$ irradiation, and subsequently $24 \mathrm{~h}$ ambient light. 
In accordance with these results, also SEM did not show significant differences between the as-assembled SNPs and the SNPs treated with UV light, nor upon prolonged exposure to ambient light. As seen in Figure 6.5, SNPs with sizes of $69 \pm 11 \mathrm{~nm}, 63 \pm 14 \mathrm{~nm}$ and $64 \pm 15 \mathrm{~nm}$ were observed after assembly, UV light irradiation, and after keeping the samples at ambient light, respectively. This reveals that UV irradiation does not influence the heteroternary complex $\mathrm{CB}[8] / \mathrm{MV} / \mathrm{Np}$ on the molecular level and therefore does not affect the SNPs.
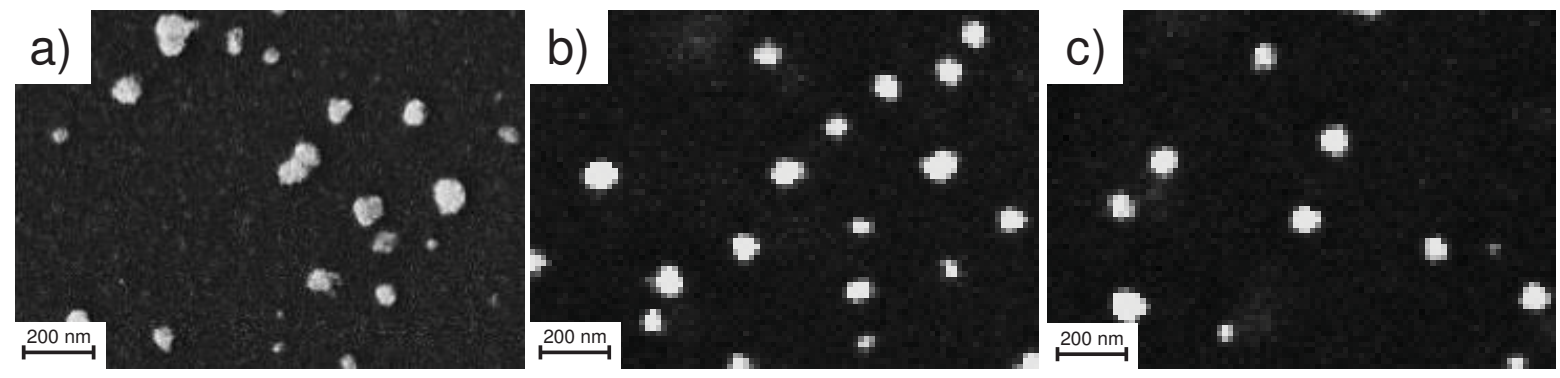

Figure 6.5: SEM images of SNPs (prepared from Np-PEG, $N p_{8}-P A M A M$ (30\% Np from Np $\left.-P A M A M\right)$, $M V-P E I$ and $C B[8]$ with $C B[8]: M V: N p=1: 1: 1)$ a) after assembly, b) after subsequent $14 h$ UV irradiation, and c) after keeping the samples for $24 \mathrm{~h}$ at ambient light.

Combined, the results presented here show that UV light can be used to trigger SNP disassembly for SNPs stabilized by CB[8]/MV/Azo interactions, whereas CB[8]/MV/Npstabilized SNPs are not affected by UV treatment. Using the photolabile Azo in the shell and the photostable $\mathrm{Np}$ in the core of the SNPs, the shell of these SNPs can be photoswitched selectively leading to aggregation of the cores.

\subsubsection{SNPs with a photoswitchable core}

To make UV light-responsive SNPs with Azo in the core, SNP assembly was carried out using $\mathrm{AzO}_{8}$-PAMAM as multivalent cross-linker and Np-PEG as monovalent stopping unit in the presence of MV-PEI and CB[8]. As shown in Scheme 6.1, it can be expected that these SNPs do not aggregate upon UV irradiation, since the SNP shell is not disrupted upon UV treatment. Moreover, UV treatment only affects the dendrimer molecules assembling in the SNP core because they are capable of trans-cis isomerization. To study the self-assembly and disassembly, and to guarantee comparability with the previously described SNPs, the core-forming $\mathrm{AzO}_{8}$-PAMAM dendrimer constituted 30\% electron-rich guest in the SNP formulation, while the Np-PEG formed the remaining $70 \%$. As for the other SNPS, SNP disassembly was triggered by UV light after confirming SNP formation, and SNP reformation was evaluated after keeping the samples subsequently at ambient light.

DLS results shown in Figure 6.6 indicate SNP formation (117 $\pm 18 \mathrm{~nm}$ ) after mixing of the supramolecular building blocks. By exposing the as-assembled SNPS to UV light, the 
observed SNP size analyzed by DLS decreased gradually to $44 \pm 22 \mathrm{~nm}$ after $14 \mathrm{~h}$. Notably, this size is comparable to the size observed for SNPs prepared using $100 \% \mathrm{~Np}$ from Np-PEG in the absence of multivalent dendrimer.

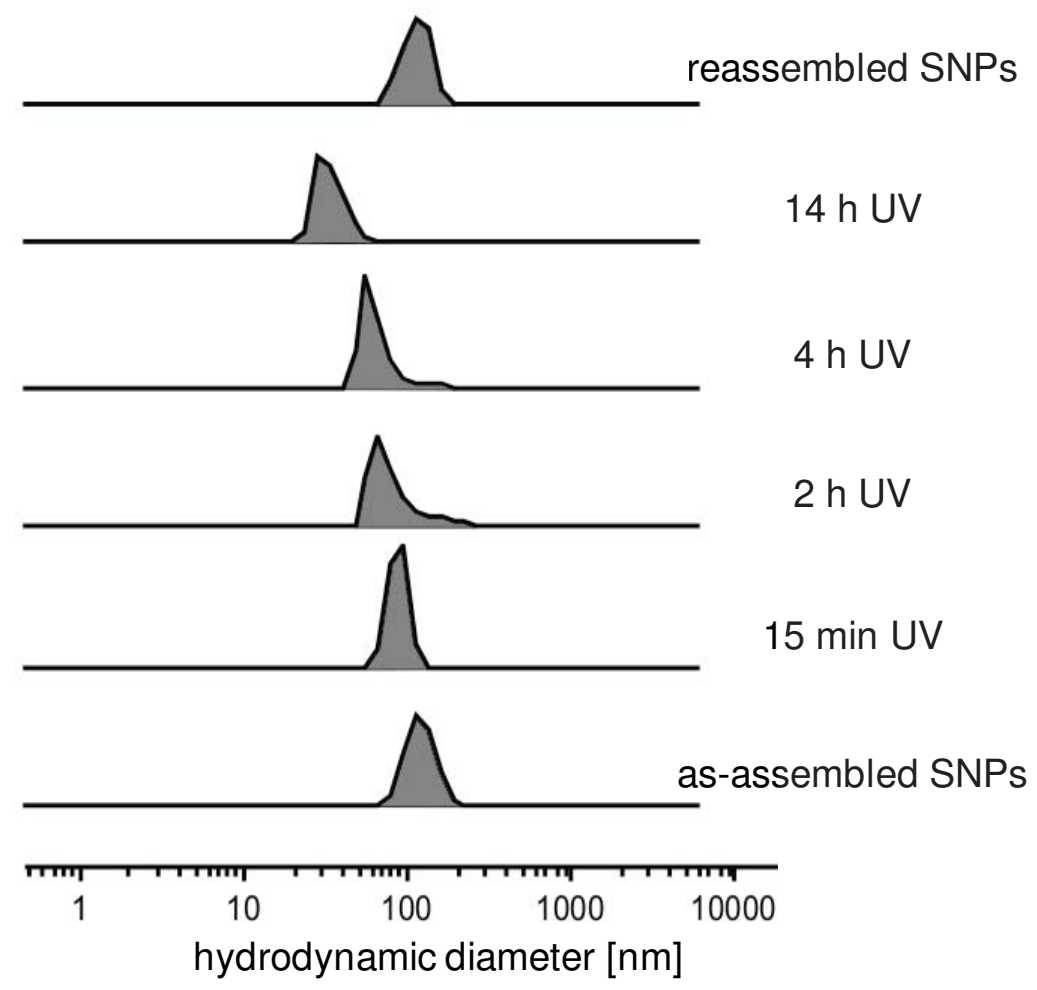

Figure 6.6: DLS size determinations of as-assembled SNPS (prepared from Np-PEG, Azos-PAMAM (Np:Azo = 70:30), MV-PEI and CB[8] with CB[8]:MV: $(N p+A z O)=1: 1: 1)$, after UV treatment for various periods of time, and after subsequent $24 \mathrm{~h}$ of ambient light.

SEM images show distinct SNPs of $80 \pm 15 \mathrm{~nm}$ after self-assembly of the four supramolecular building blocks (Figure 6.7). The size and appearance of the SNPs were changed strongly after UV light exposure. The SNPs showed a dense core surrounded by less dense organic material. The average SNP size was $47 \pm 10 \mathrm{~nm}$, which is significantly smaller than before irradiation. Keeping the samples at ambient light resulted in SNPS with an average size of $68 \pm 19 \mathrm{~nm}$, which indicates that the SNPs regained most of their original size but not completely. Furthermore, the SNP shell did not look as well-defined as observed for the SNPS prior to UV treatment, suggesting the samples may require more time for complete reintegration of all $\mathrm{AzO}_{8}$-PAMAM. 

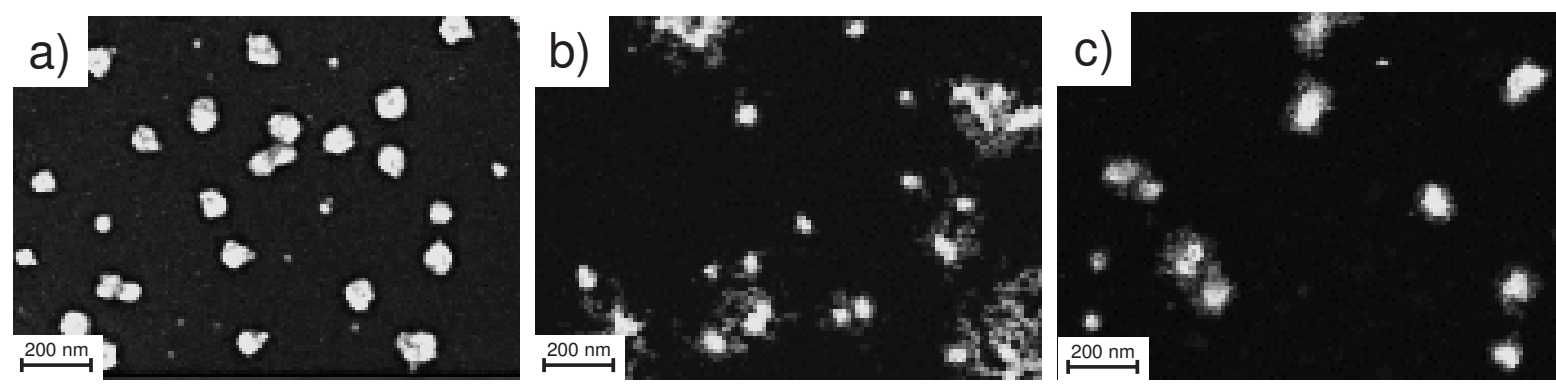

Figure 6.7: SEM images of SNPS (prepared from Np-PEG, Azos-PAMAM (Np:Azo = 70:30), MV-PEI and $C B[8]$ with $C B[8]: M V:(N p+A z O)=1: 1: 1)$ a) after self-assembly, b) after $14 \mathrm{~h}$ UV light, c) after subsequent $24 \mathrm{~h}$ ambient light.

Summarizing, SNPs stabilized by multivalent $\mathrm{CB}[8] / \mathrm{MV} / \mathrm{Azo}$ interactions in the core and by monovalent $\mathrm{CB}[8] / \mathrm{MV} / \mathrm{Np}$ interactions in the shell shrink gradually upon UV treatment. This SNP disintegration can be ascribed to the destabilization of CB[8]/MV/Azo interactions in the core by photoisomerization of the Azo going along with a release of photoswitched $\mathrm{AzO}_{8}$-PAMAM, whereas the SNP shell with unaffected Np-containing ternary complexes persists. In comparison to the SNPs whereby photoswitching of the shell leads to aggregation, the disintegration process of the core-switching SNPs is rather slow: only a minor change was observed by DLS for samples treated for 15 min (111 \pm $25 \mathrm{~nm}$ ) and $1 \mathrm{~h}$ with UV light (102 $\pm 25 \mathrm{~nm}$ ). This slow UV response can be explained by the incomplete trans-cis isomerization described in Chapter 5, whereby switching of the $\mathrm{CB}[8] / \mathrm{MV} / \mathrm{Azo}$ ternary complexes linked to the multivalent $\mathrm{AzO}_{8}$-PAMAM provides dendrimers that still have a few non-switched and interacting Azo groups. This leads to a continuous, but rather slow release of dendrimer from the SNP core, while the SNPS remain continuously stabilized by the monovalent Np-PEG heteroternary complexes.

\subsection{Conclusions}

In summary, we have presented here a toolbox approach for the fabrication of SNPs stabilized by the heteroternary complexes $\mathrm{CB}[8] / \mathrm{MV} / \mathrm{Np}$ and $\mathrm{CB}[8] / \mathrm{MV} / \mathrm{Azo}$, allowing the placement of photoresponsive Azo groups in the SNP shell, the core or both. SNP formation appeared to be independent of the use of Azo or Np guest moieties, and similar SNP sizes were observed for all SNP formulations. Depending on the choice of the mono and multivalent building blocks four different types of SNPs were prepared, all of which respond differently to UV light. Whereas non-responsive SNPs were formed by using $\mathrm{CB}[8] / \mathrm{MV} / \mathrm{Np}$ interactions exclusively, which SNPs may be useful as passive carrier in biomedical applications, photoisomerization of Azo going along with rupture of the hostguest complexes triggers different SNP disassembly pathways of the SNPs depending on the location of the switchable Azo moiety. 
SNPs with a switchable shell aggregated relatively fast upon UV treatment, and could be useful as drug carriers in oncology as SNP aggregation could trap the SNPs in tumor tissue in an enhanced EPR effect. In contrast, SNPs with a switchable core shrank slowly upon UV treatment due to release of the multivalent dendrimer from the SNP core. This SNP formulation might be suited for drug delivery applications in which continuous drug release is required over the course of hours. A combination of both (transient) aggregation and disassembly was observed for the initially reported SNPs with photoresponsive Azo in both the shell and the core, which might be beneficial for drug release in tumor tissue. Overall, this study shows that the degradation rate and pathway of SNPs can be pre-programmed by selecting the correct SNP architecture.

\subsection{Acknowledgements}

Jens Voskuhl is gratefully acknowledged for the synthesis of $\mathrm{AzO}_{8}$-PAMAM and for fruitful discussions.

\subsection{Experimental Section}

\subsubsection{Materials}

Cucurbit[8]uril (CB[8]) was purchased from Strem Chemicals and its purity was assessed by microcalorimetric titration against paraquat. The synthesis and characterization of $\mathrm{Np}$ PEG $(5000 \mathrm{~g} / \mathrm{mol}), \mathrm{Np}_{8}$-PAMAM, MV-PEI, Azo-PEG $(5000 \mathrm{~g} / \mathrm{mol})$ and Azo - PAMAM is described in Chapter 3 and Chapter 5. SNP preparation was carried out in water of MilliQ quality (Millipore, $R=18.2 \mathrm{M} \Omega \mathrm{cm}^{-1}$ ).

\subsubsection{Methods}

Supramolecular nanoparticle assembly

For the preparation of SNPs stabilized by heteroternary host-guest interactions, solutions of Azo-PEG $(1.96 \mu \mathrm{M})$, Np-PEG $(1.96 \mu \mathrm{M})$, MV-PEI $(0.31 \mu \mathrm{M})$ and $\mathrm{CB}[8](2.8 \mu \mathrm{M})$ in water and $\mathrm{AzO}_{8}$-PAMAM $(2.63 \mu \mathrm{M})$ and $\mathrm{Np}_{8}$-PAMAM $(2.63 \mu \mathrm{M})$ in DMSO were prepared. The SNPs were formed by mixing $500 \mu \mathrm{L}$ MV-PEI, $250 \mu \mathrm{L}$ CB[8], $250 \mu \mathrm{L}$ Azo-PEG or Np-PEG and $10 \mu \mathrm{L}_{\mathrm{Z} \mathrm{O}_{8}}$-PAMAM or $\mathrm{Np}_{8}$-PAMAM. The samples were kept at RT for 2 days before DLS and SEM analysis.

\section{UV irradiation of the SNPS}

UV irradiation experiments were carried out with a Hönle bluepoint 2 easycure using a shortpass filter $\lambda \leq 400 \mathrm{~nm}$. After formation, the SNPs were treated with UV light for different periods. Therefore the samples were filled in a quartz cuvette and exposed to light with $\lambda \leq 400 \mathrm{~nm}$. To analyze the ongoing disassembly process, the samples were analyzed by DLS in dark and UV irradiation was continued. 
The SNP reassembly was evaluated after keeping the UV treated SNP samples for $24 \mathrm{~h}$ at ambient light, prior to analysis by DLS and SEM.

\subsubsection{Equipment}

\section{DLS analysis (DLS)}

DLS experiments were performed with a Nanotrac Wave (Microtrac) at $25^{\circ} \mathrm{C}$ using a laser wavelength of $780 \mathrm{~nm}$ at a scattering angle of $90^{\circ}$. The reported SNP hydrodynamic diameters are averaged of at least three individual measurements. The DLS curves shown in this chapter are representative for the respective systems studied here. For UV-treated samples, the measurements were carried out in the dark directly after irradiation.

\section{Scanning electron microscopy (SEM)}

All SEM images were taken with a Carl Zeiss Merlin scanning electron microscope. For sample preparation, the aqueous SNP solutions were drop-cast on a Formvar coated copper TEM grid. The solution was dried and analyzed without further treatment of the samples. For the samples measured after UV irradiation, the drying process was carried out in the dark.

\subsection{References}

1. L. Brannon-Peppas and J. O. Blanchette, Advanced Drug Delivery Reviews, 2012, 64, Supplement, 206-212.

2. S. Parveen, R. Misra and S. K. Sahoo, Nanomedicine: Nanotechnology, Biology and Medicine, 2012, 8, 147-166.

3. S. M. Janib, A. S. Moses and J. A. MacKay, Advanced Drug Delivery Reviews, 2010, 62, 1052-1063.

4. X. Ma, Y. Zhao and X.-J. Liang, Accounts of Chemical Research, 2011, 44, 1114-1122.

5. N. Sanvicens and M. P. Marco, Trends in Biotechnology, 2008, 26, 425-433.

6. M. De, P. S. Ghosh and V. M. Rotello, Advanced Materials, 2008, 20, 4225-4241.

7. W. J. Stark, Angewandte Chemie International Edition, 2011, 50, 1242-1258.

8. C. S. Kim, B. Duncan, B. Creran and V. M. Rotello, Nano Today, 2013, 8, 439-447.

9. K. Loomis, K. McNeeley and R. V. Bellamkonda, Soft Matter, 2011, 7, 839-856.

10. M. Delcea, H. Möhwald and A. G. Skirtach, Advanced Drug Delivery Reviews, 2011, 63, 730-747.

11. Q. He and J. Shi, Journal of Materials Chemistry, 2011, 21, 5845-5855.

12. I. I. Slowing, J. L. Vivero-Escoto, C.-W. Wu and V. S. Y. Lin, Advanced Drug Delivery Reviews, 2008, 60, 1278-1288.

13. C. Minelli, S. B. Lowe and M. M. Stevens, Small, 2010, 6, 2336-2357.

14. E. K. U. Larsen, T. Nielsen, T. Wittenborn, H. Birkedal, T. Vorup-Jensen, M. H. Jakobsen, L. Østergaard, M. R. Horsman, F. Besenbacher, K. A. Howard and J. Kjems, ACS Nano, 2009, 3, 1947-1951.

15. J. Nam, N. Won, H. Jin, H. Chung and S. Kim, Journal of the American Chemical Society, 2009, 131, 13639-13645. 
16. X. Liu, Y. Chen, H. Li, N. Huang, Q. Jin, K. Ren and J. Ji, ACS Nano, 2013, 7, 6244-6257.

17. J. Sharma, R. Chhabra, H. Yan and Y. Liu, Chemical Communications, 2007, 477-479.

18. S. Basu, S. K. Ghosh, S. Kundu, S. Panigrahi, S. Praharaj, S. Pande, S. Jana and T. Pal, Journal of Colloid and Interface Science, 2007, 313, 724-734.

19. J.-Z. Du, X.-J. Du, C.-Q. Mao and J. Wang, Journal of the American Chemical Society, 2011, 133, 17560-17563.

20. H. Meng, M. Xue, T. Xia, Y.-L. Zhao, F. Tamanoi, J. F. Stoddart, J. I. Zink and A. E. Nel, Journal of the American Chemical Society, 2010, 132, 12690-12697.

21. P. Pradhan, J. Giri, F. Rieken, C. Koch, O. Mykhaylyk, M. Döblinger, R. Banerjee, D. Bahadur and C. Plank, Journal of Controlled Release, 2010, 142, 108-121.

22. W. Wang, H. Sun, F. Meng, S. Ma, H. Liu and Z. Zhong, Soft Matter, 2012, 8, 3949-3956.

23. A. W. Jackson and D. A. Fulton, Macromolecules, 2012, 45, 2699-2708.

24. C. Chen, J. Geng, F. Pu, X. Yang, J. Ren and X. Qu, Angewandte Chemie International Edition, 2011, 50, 882-886.

25. X. Huang, X. Jiang, Q. Yang, Y. Chu, G. Zhang, B. Yang and R. Zhuo, Journal of Materials Chemistry $B$, 2013, 1, 1860-1868.

26. J. E. Tengood, I. S. Alferiev, K. Zhang, I. Fishbein, R. J. Levy and M. Chorny, Proceedings of the National Academy of Sciences, 2014, 111, 4245-4250.

27. D. Han, X. Tong and Y. Zhao, Langmuir, 2012, 28, 2327-2331.

28. Y. Ping, Q. Hu, G. Tang and J. Li, Biomaterials, 2013, 34, 6482-6494.

29. J.-H. Lee, K.-J. Chen, S.-H. Noh, M. A. Garcia, H. Wang, W.-Y. Lin, H. Jeong, B. J. Kong, D. B. Stout, J. Cheon and H.-R. Tseng, Angewandte Chemie International Edition, 2013, 52, 4384-4388.

30. J. Zhang, K. Ellsworth and P. X. Ma, Journal of Controlled Release, 2010, 145, 116-123.

31. M. E. Davis, J. E. Zuckerman, C. H. J. Choi, D. Seligson, A. Tolcher, C. A. Alabi, Y. Yen, J. D. Heidel and A. Ribas, Nature, 2010, 464, 1067-1070.

32. A. Kulkarni, W. Deng, S.-h. Hyun and D. H. Thompson, Bioconjugate Chemistry, 2012, 23, 933-940.

33. S. Eliasof, D. Lazarus, C. G. Peters, R. I. Case, R. O. Cole, J. Hwang, T. Schluep, J. Chao, J. Lin and Y. Yen, Proceedings of the National Academy of Sciences, 2013, 110, 1512715132.

34. K.-J. Chen, L. Tang, M. A. Garcia, H. Wang, H. Lu, W.-Y. Lin, S. Hou, Q. Yin, C. K. F. Shen, J. Cheng and H.-R. Tseng, Biomaterials, 2012, 33, 1162-1169.

35. J. Zhang and P. X. Ma, Angewandte Chemie International Edition, 2009, 48, 964-968.

36. E. Battistini, E. Gianolio, R. Gref, P. Couvreur, S. Fuzerova, M. Othman, S. Aime, B. Badet and P. Durand, Chemistry - A European Journal, 2008, 14, 4551-4561.

37. K.-J. Chen, S. M. Wolahan, H. Wang, C.-H. Hsu, H.-W. Chang, A. Durazo, L.-P. Hwang, M. A. Garcia, Z. K. Jiang, L. Wu, Y.-Y. Lin and H.-R. Tseng, Biomaterials, 2011, 32, 21602165.

38. F. Tian, D. Jiao, F. Biedermann and O. A. Scherman, Nature Communications, 2012, 3, 1207.

39. U. Rauwald, F. Biedermann, S. p. Deroo, C. V. Robinson and O. A. Scherman, Journal of Physical Chemistry B, 2010, 114, 8606-8615. 


\section{Chapter 7}

\section{Zwitterionic Supramolecular Nanoparticles: Self-Assembly and Responsive Properties}

Supramolecular nanoparticles (SNPs) are of high interest in both nanoscience and molecular diagnostics and therapeutics, because of their reversible and designable properties. To ensure colloidal stabilization and biocompatibility, most reported strategies require the use of hydrophilic long-chain polymers such as poly(ethylene glycol). Here, we show the formation of zwitterionic supramolecular nanoparticles (ZSNPs) from appropriately functionalized mono- and multivalent components, based on the heteroternary host-guest complexation between cucurbit[8]uril (CB[8]), methyl viologen (MV), and azobenzene (Azo), while using the monovalent, small-molecule, non-fouling Azo-carboxybetaine analogue (Azo-Zwit) as the shell-forming component. Even though steric shell stabilization is absent, the zwitterionic Azo-Zwit ensures stability of the ZSNPS in water, in PBS ( $\mathrm{pH} \mathrm{7.4)}$ at ionic strengths ranging from 0-700 mM, and in PBS containing BSA. Size tuning by control over the stoichiometry of the components, as well as reversible assembly and disassembly by photoisomerization of the Azo moieties were observed. Surprisingly, the ZSNPs exhibited aggregation at the narrow pH range of 6.26.8, which holds promise for an enhanced EPR effect when considering the ZSNPs as potential cancer therapeutics. 


\subsection{Introduction}

Nanoparticles (NPS) are of major interest because of their size-dependent chemical and physical properties. Next to catalytic, optical, sensor or electronic applications, the use of NPs in biomedicine has gained intense scientific interest over the past two decades. ${ }^{1,2}$ In oncology, for example, countless studies have been carried out on the targeted delivery of active compounds. ${ }^{3}$ Based on the enhanced permeability and retention (EPR) effect caused by vascular hyperpermeability and impaired lymphatic drainage, NPs have been used to accumulate drugs and imaging agents in the tumor tissue. ${ }^{4,5}$ The microenvironment of this tissue, in particular the more acidic extracellular $\mathrm{pH}$ (6.0-7.0) compared to healthy tissue, has enabled the selective release of active compounds using responsive drug delivery vehicles or has initiated NP aggregation to enhance their retention in tumor tissue. ${ }^{6}$

A general problem of the administration of NPs to the human body is the rapid clearance of the NPs from the blood stream by the reticuloendothelial system (RES) or mononuclear phagocytic system (MPS). ${ }^{7}$ This process is induced by opsonization, the non-specific binding of proteins to NP epitopes, resulting in NP aggregation and elimination of the NPs by the immune defense system. ${ }^{7,8}$ In general, these processes are strongly dependent on the surface properties of the NPs. To enhance the pharmacokinetics and biodistribution of NPs in vivo, a poly(ethylene glycol) (PEG) coating is commonly used. ${ }^{9,10}$ However, PEG degradation by oxidation can result in loss of anti-fouling properties upon prolonged usage or storage. ${ }^{11,12}$

Recently, a new class of non-fouling materials based on zwitterions has been put forward as an alternative for PEG in the functionalization of biomedically relevant materials. ${ }^{13,14}$ Zwitterions are molecules carrying equimolar amounts of negative and positive charges guaranteeing overall charge neutrality. Due to their ionic character, zwitterion-bearing compounds are ultra-hydrophilic. Various naturally occurring membrane lipids are of zwitterionic origin and have shown to be strongly anti-fouling when assembled in a model bilayer. ${ }^{15}$ Jiang and coworkers have used zwitterionic compounds for the coating of different types of NPs. ${ }^{16-18}$ In these studies, it was shown that a carboxybetaine shell around the NPs prevents non-specific protein binding in undiluted blood serum.

Soft supramolecular nanoparticles (SNPs) are promising materials because their formation strategy results in stable, yet reversible nanovectors. A variety of biomedically relevant SNPs based on host-guest chemistry has been developed by including active compounds into the SNP formulation. Drug delivery SNPs have been formed by covalent modification of the supramolecular building blocks with chemotherapy drugs such as camptothecin $^{19}$ and doxorubicin, ${ }^{20}$ whereas gene delivery vehicles have been based on electrostatic interactions of positively charged building blocks and negatively charged 
RNA. ${ }^{21}$ Despite the chemical flexibility of SNP approaches, almost all SNP formulations require a PEG coating to ensure colloidal stabilization ${ }^{22,23}$ in aqueous media or to inhibit aggregation by interactions with proteins in the blood flow. ${ }^{21}$ Not only the hydrophilic properties of PEG, but also steric stabilization by the long polymeric PEG chains are believed to be important for achieving these properties.

Here, we describe the formation, stability and responsive properties of zwitterionic supramolecular nanoparticles (ZSNPs). The zwitterionic motif proposed here, is an alternative, shell-forming moiety around the SNP core to provide colloidal stability and anti-fouling properties. Hereto, SNPs are designed based on the heteroternary host-guest interaction between cucurbit[8]uril (CB[8]), methyl viologen (MV) and azobenzene (Azo) (Scheme 7.1). The monovalent character of the non-fouling Azo-Zwit is required to avoid uncontrolled growth of the SNP core by assembly of the multivalent components. An important research question is whether the relatively short zwitterionic molecule, which is much shorter than the typically used polymeric PEG molecules, provides sufficient colloidal stability in aqueous solution, in the absence or presence of relevant concentrations of serum proteins. Furthermore, stoichiometry-based size control, UVinitiated rupture by switching of the Azo moieties, and the stability of the SNPs in different $\mathrm{pH}$ media are also described. 
a)

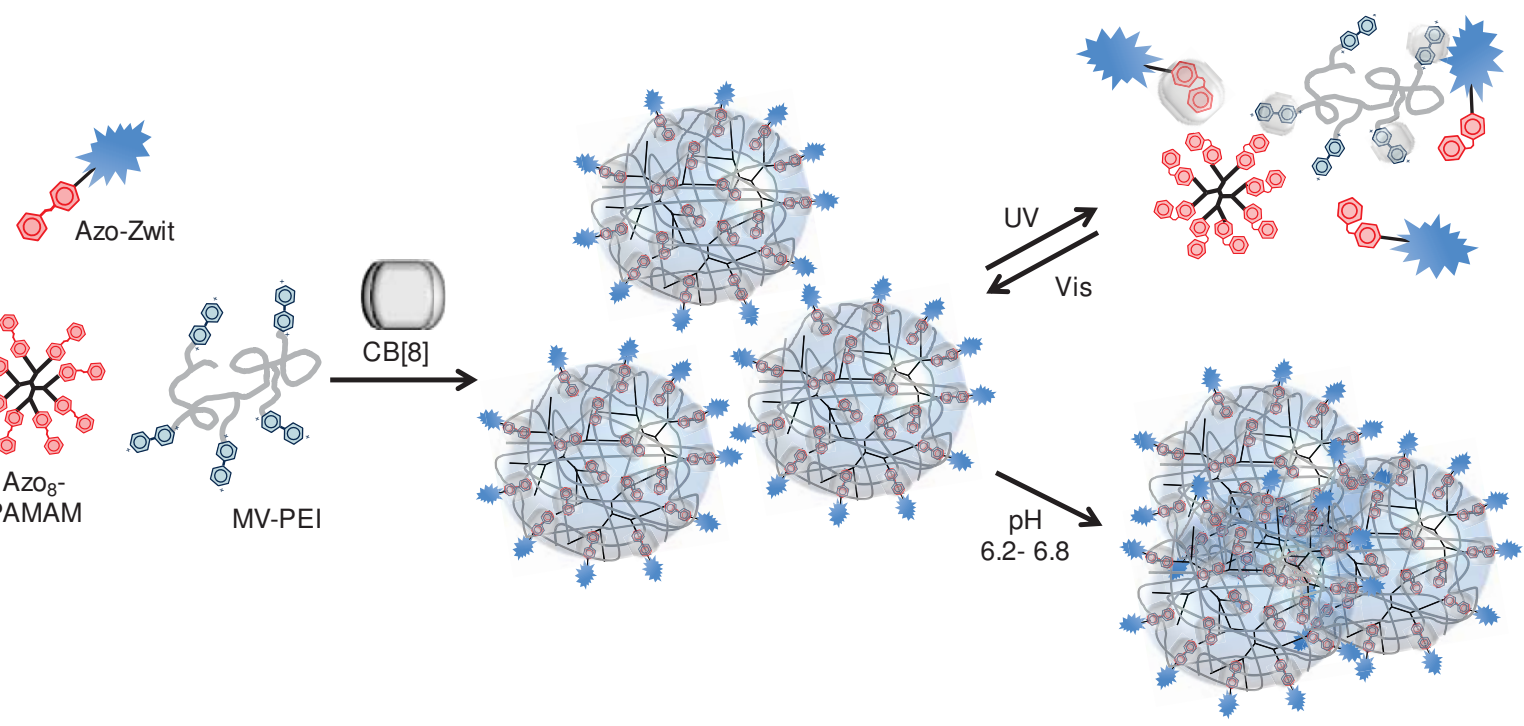

b)
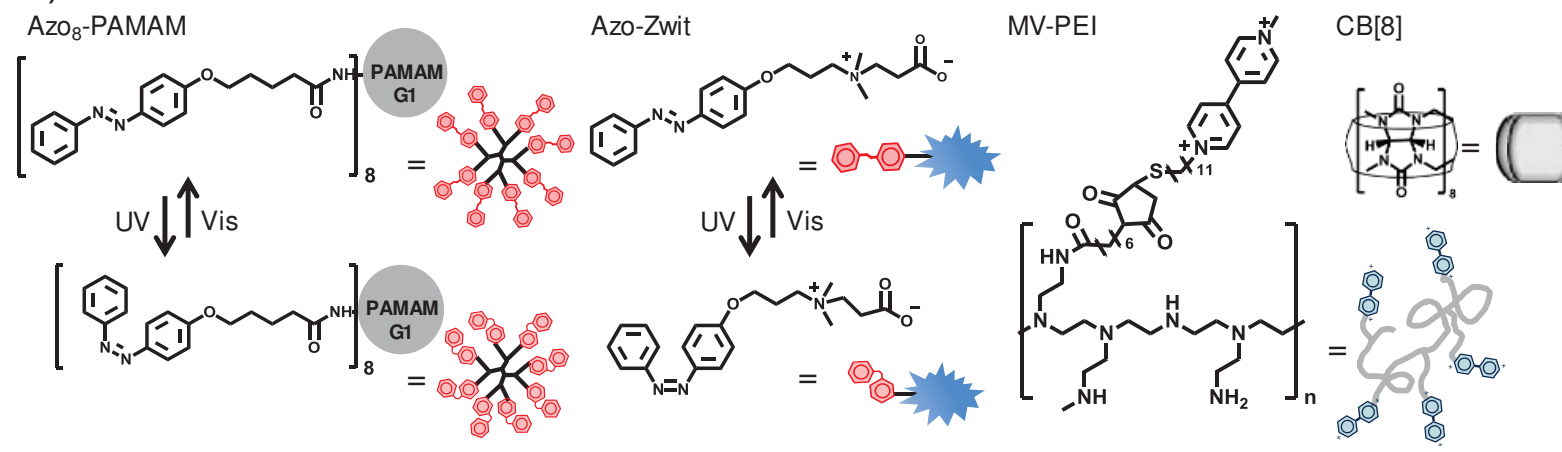

Scheme 7.1: a) Schematic illustration of the self-assembly of zwitterionic supramolecular nanoparticles (ZSNPS) mediated by ternary host-guest complexes between cucurbit[8]uril (CB[8]), methyl viologen (MV) and azobenzene (Azo), UV light-triggered SNP disassembly and pH-responsive SNP aggregation. b) Supramolecular components involved in ZSNP assembly: azobenzenefunctionalized poly(amidoamine) dendrimer (Azos-PAMAM), a monovalent Azo-carboxybetaine analogue (Azo-Zwit), MV-functionalized poly(ethylene imine) (MV-PEI), and CB[8].

\subsection{Results and Discussion}

\subsubsection{Supramolecular nanoparticle formation in different media}

An Azo-carboxybetaine analogue (Azo-Zwit) was synthesized (see Scheme 7.2) and used as monovalent capping ligand in the formation of the ZSNPs. By mixing MV-substituted poly(ethylene imine) (MV-PEI, degree of substitution: 4.5 MV units per polymer chain, $\mathrm{M}_{\mathrm{W}}$ $10 \mathrm{kDa}$, Chapter 3), Azo-terminated poly(amidoamine) dendrimer generation 1 ( $\mathrm{AzO}_{8}$ PAMAM, Chapter 5), Azo-Zwit and $\mathrm{CB}[8]$, SNPs are formed by multiple heteroternary complexes between $\mathrm{CB}[8], \mathrm{MV}$ and trans-Azo. The core of these SNPs is assembled via multivalent intermolecular interactions between the MV-polymer and the Azo dendrimer in the presence of $\mathrm{CB}[8]$, whereas monovalent interactions involving Azo-Zwit constitute the shell. 


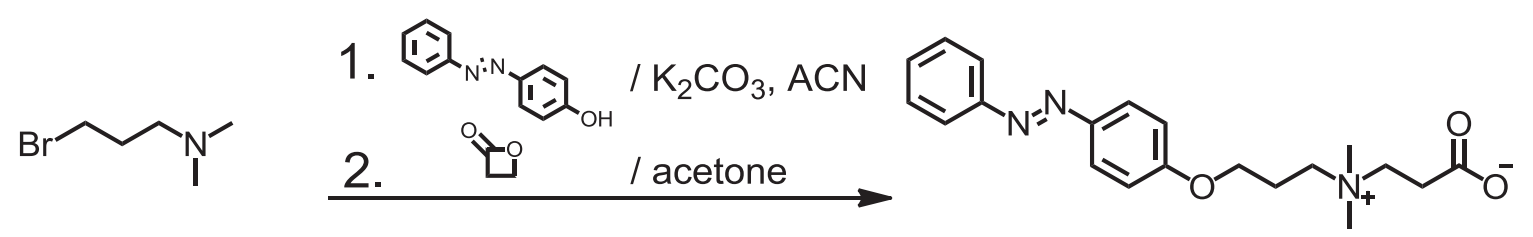

Scheme 7.2: Synthetic route towards the azobenzene-functionalized zwitterion derivative Azo-Zwit used in this study.

The formation of ZSNPs was studied in water at an equimolar 1:1:1 ratio of the molecular recognition moieties CB[8], MV and Azo. Using 70\% Azo derived from AzoZwit and $30 \%$ from Azo $_{8}$-PAMAM, ZSNPs with an average size of $78 \pm 9 \mathrm{~nm}$ and an average hydrodynamic diameter of $133 \pm 19 \mathrm{~nm}$ were observed by scanning electron microscopy (SEM) and dynamic light scattering (DLS), respectively (see Figure 7.1a-b). The ZSNP sizes observed by SEM are smaller than by DLS which is attributed to (i) ZSNP shrinkage during SEM sample preparation by the loss of water and (ii) DLS reflecting the hydrodynamic diameter of the ZSNPs.
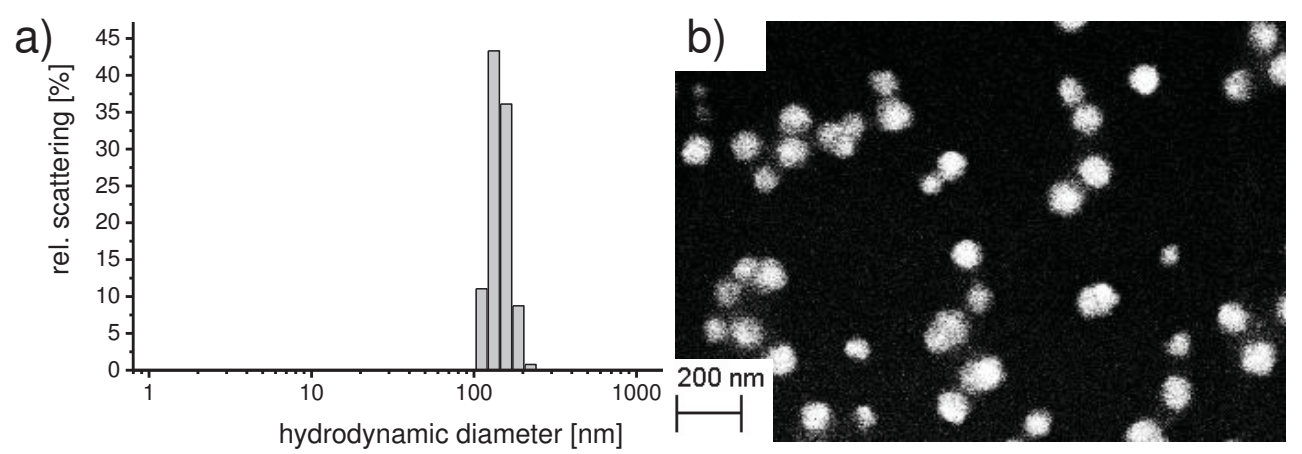

Figure 7.1: Size determination by a) DLS and b) SEM of ZSNPS prepared with CB[8]:MV:AZO = 1:1:1, with $30 \%$ Azo from $\mathrm{AzO}_{8}-$ PAMAM prepared in water.

To verify that the ternary host-guest complexation is essential for stable ZSNP formation, different control experiments were carried out. Congruently, ZSNP formation was detectable neither for samples prepared in the absence of $\mathrm{CB}[8]$ or MV-PEI nor for samples prepared with $\mathrm{CB}[7]$ instead of $\mathrm{CB}[8]$ (see Figure 7.2). It is known, that the smaller $\mathrm{CB}[7]$ is too small to encapsulate both guest molecules. These results confirm that ZSNP formation is induced by ternary host-guest complexation between MV, Azo and $\mathrm{CB}[8]$. 

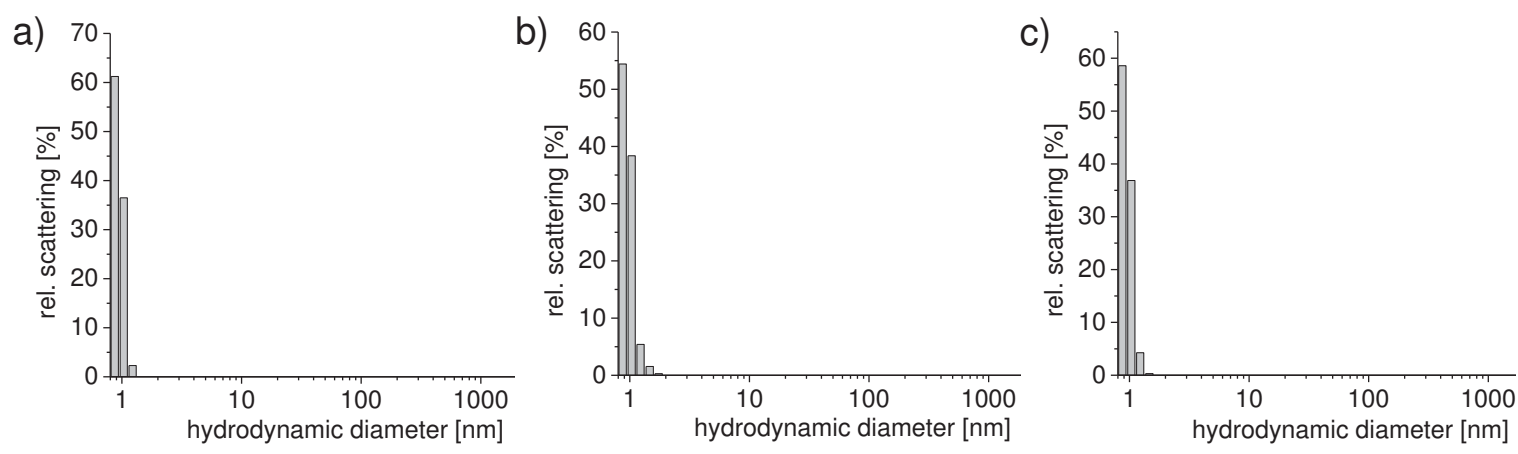

Figure 7.2: DLS analysis of ZSNP solutions (30\% Azo from Azo8-PAMAM, in water): a) in the absence of $C B[8], b)$ in the absence of $M V-P E I, C)$ in the presence of $C B[7]$ instead of $C B[8]$.

The use of NPs for biomedically relevant applications requires NP stability at physiological ionic strength. Therefore, ZSNP self-assembly was evaluated in phosphate-buffered saline (PBS, $140 \mathrm{mM} \mathrm{KCl} \mathrm{pH} \mathrm{7.4).} \mathrm{Using} \mathrm{the} \mathrm{same} \mathrm{ZSNP} \mathrm{composition,} \mathrm{SEM} \mathrm{and} \mathrm{DLS}$ showed negligible size and morphology differences in comparison to experiments carried out in water (see Figure 7.3). This observation clearly shows that stable ZSNPs have been formed in PBS. In contrast, SNP formation experiments carried out with MV-PEI, $\mathrm{AzO}_{8}$-PAMAM, CB[8] and Azo-functionalized tri(ethylene glycol) (Azo-TEG) in PBS, showed irreproducible results and uncontrolled aggregation by DLS. Similarly, SEM (Figure 7.4) showed indistinct structures, which are incomparable to the ZSNP structures reported here or to previously observed SNPs prepared by polymeric Azo-PEG ( $M_{W} 5$ $\mathrm{kDa}$ ) as shown in Chapter 5 . This illustrates that the monovalent Azo-Zwit provides sufficient colloidal stability to the ZSNPs in PBS, whereas Azo-TEG with a comparable molecular length does not. Most likely, the ionic character of the Azo-Zwit renders the ZSNP particle shell ultra-hydrophilic, obviating the steric stabilization known for PEGmodified SNPs.

a)

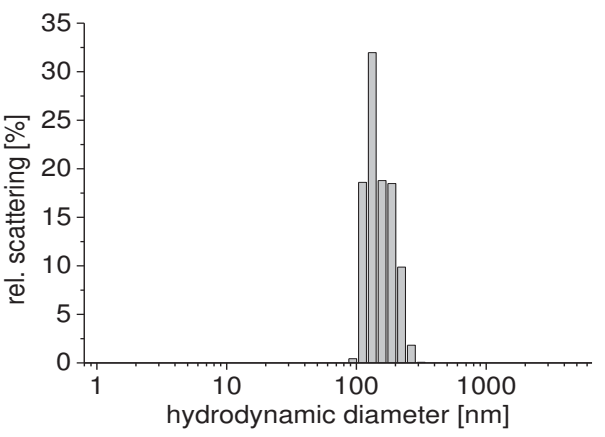

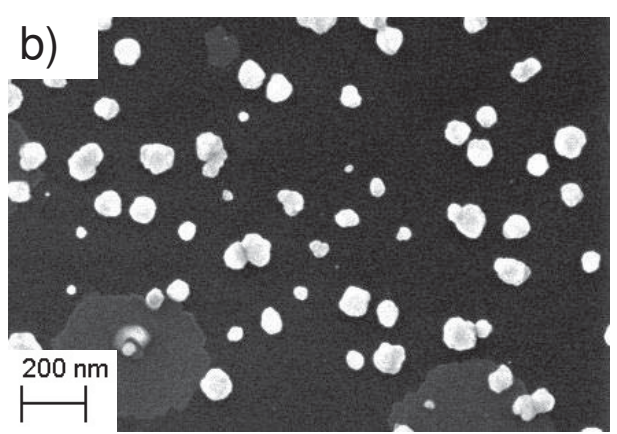

Figure 7.3: Size determination by a) DLS and b) SEM of ZSNPS prepared with CB[8]:MV:Azo = 1:1:1, with 30\% Azo from $\mathrm{Azo}_{8}$-PAMAM prepared in PBS (140 mM KCl, $\mathrm{pH}$ 7.4). 

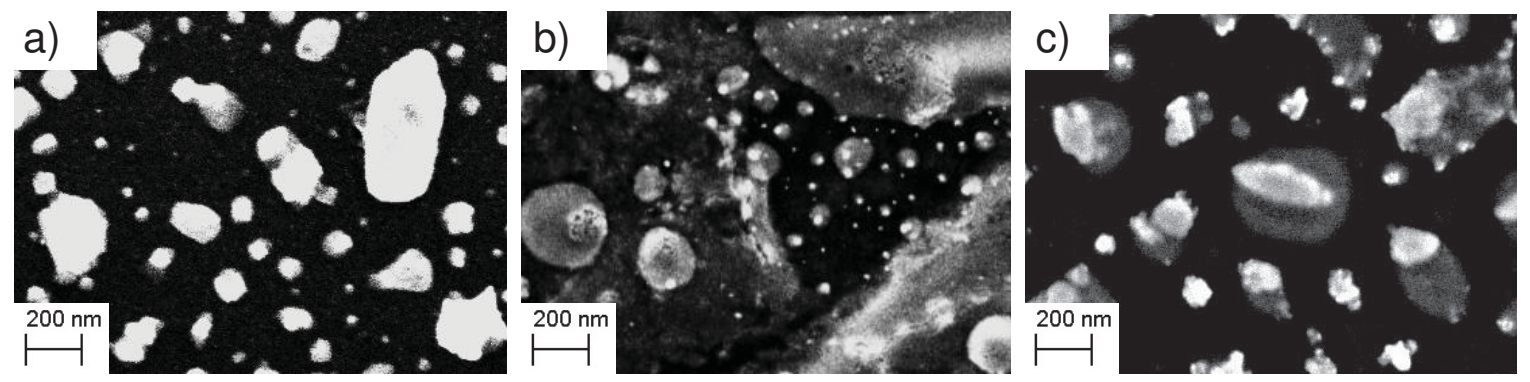

Figure 7.4: Size determination by SEM of different SNP formulations prepared with Azo-tri(ethylene glycol): a) $10 \%$ Azo from $\mathrm{AzO}_{8}-\mathrm{PAMAM}$, b) $20 \% \mathrm{Azo}$ from $\mathrm{Azo}_{8}-\mathrm{PAMAM}$, and c) $30 \%$ Azo from $\mathrm{Azo}_{8^{-}}$ PAMAM.

To investigate the effect of ionic strength on ZSNP formation and stability, self-assembly of the four supramolecular building blocks was carried out in PBS with varying concentrations of $\mathrm{KCl}(50-700 \mathrm{mM})$ using $30 \%$ Azo from $\mathrm{Azo}_{8}$-PAMAM. DLS and SEM revealed that ZSNPs are formed regardless of the ionic strength (see Figures 7.3 and 7.5). The ZSNPs were stable for at least 5 days after self-assembly. Nevertheless, the ZSNP size and size distribution observed for the samples prepared in PBS with $700 \mathrm{mM}$ $\mathrm{KCl}$ were higher ( $237 \pm 42 \mathrm{~nm}$ by DLS and $117 \pm 41 \mathrm{~nm}$ by SEM) as compared to the ZSNPs at $140 \mathrm{mM} \mathrm{KCl}(154 \pm 13 \mathrm{~nm}$ by DLS and $72 \pm 17 \mathrm{~nm}$ by SEM). We have recently shown that SNP destabilization at high ionic strength can be inhibited by a monovalent capping PEG ( $\left.M_{\mathrm{W}} 5 \mathrm{kDa}\right){ }^{24}$ The results shown here indicate that Azo-Zwit is also capable of preserving colloidal stability at high ionic strength.
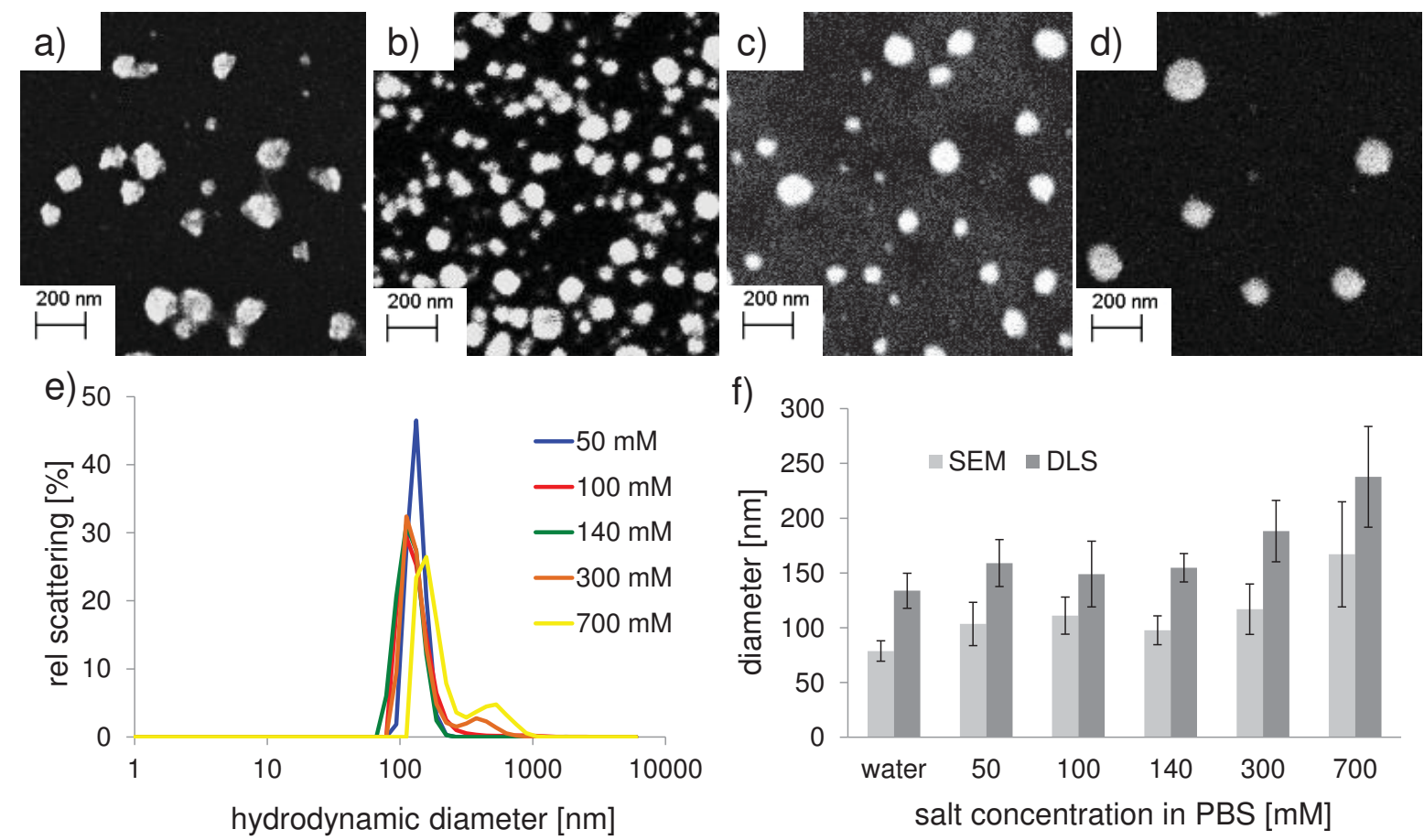

f) 300

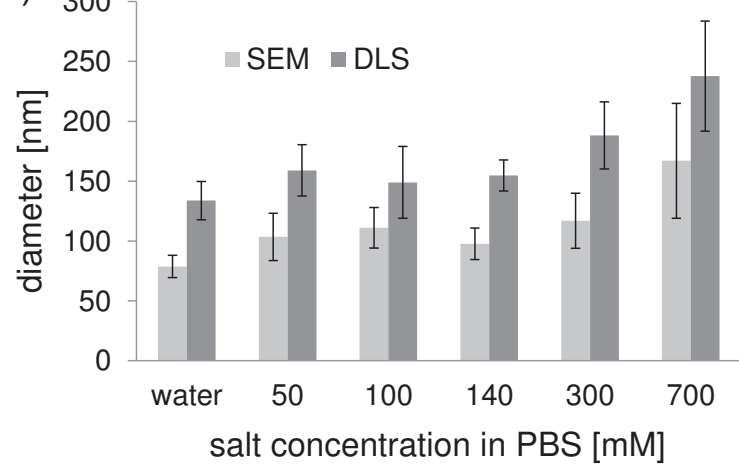

Figure 7.5: Size determination of ZSNPs prepared in PBS ( $\mathrm{pH}$ 7.4) with different $\mathrm{KCl}$ concentrations: a-d) SEM images at ionic strengths of: $50 \mathrm{mM}$, b) $100 \mathrm{mM}$, c) $300 \mathrm{mM}$, d) $700 \mathrm{mM}$, e) DLS curves for the different SNP formulations. f) ZSNPS diameters as measured by SEM ( $($ )and DLS ( $\square$ ). 
Importantly, ZSNPs were also stable after mixing $250 \mu \mathrm{L}$ ZSNPs solution with $500 \mu \mathrm{L}$ of a 5 wt\% dispersion of BSA in PBS, which is the common concentration of serum proteins in blood. DLS gave inconclusive results because of the high protein concentration, but SEM images unambiguously revealed the stability of the ZSNPs in this medium (Figure 7.6).
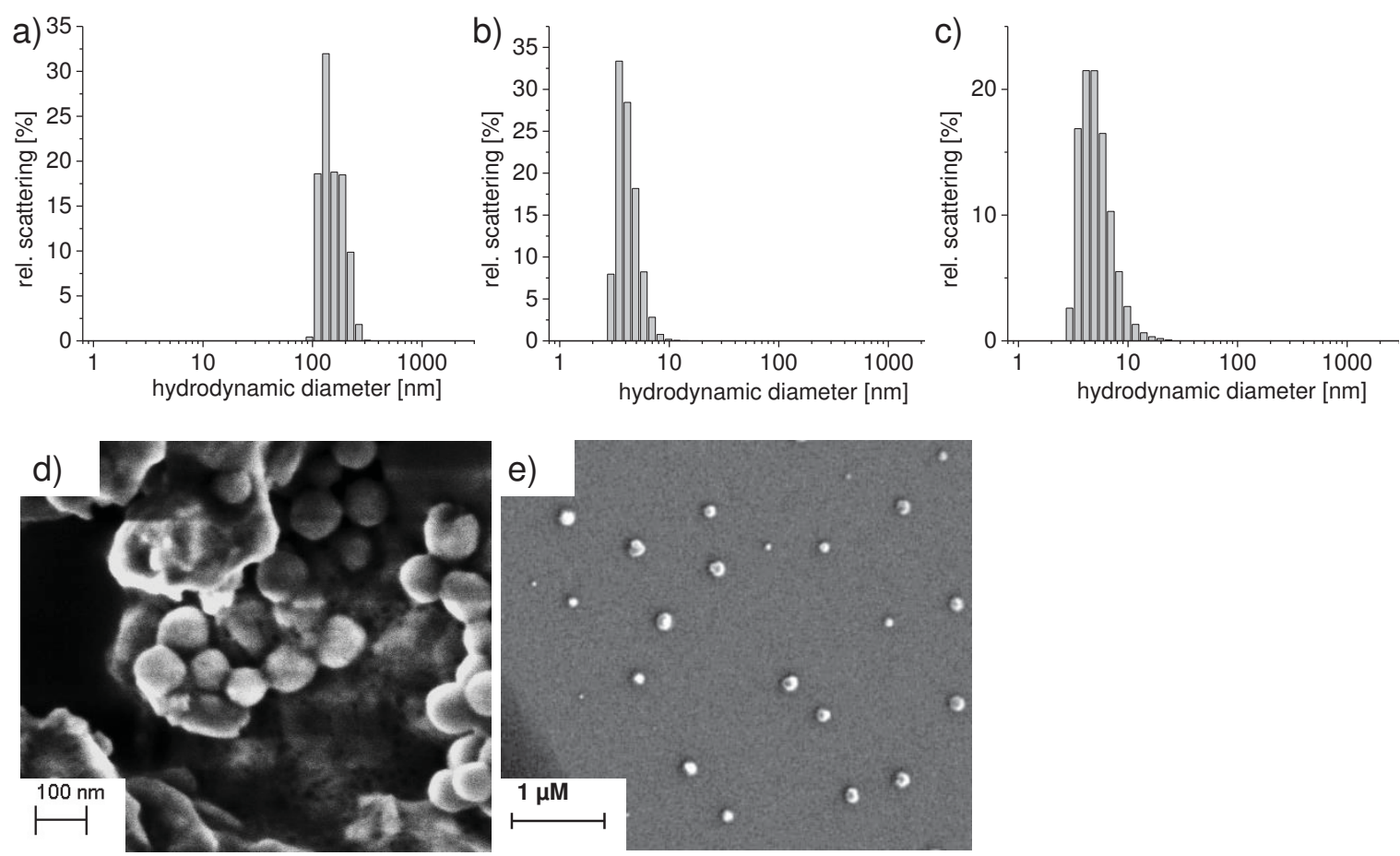

Figure 7.6: Size determination of ZSNPS prepared in PBS ( $\mathrm{PH} 7.4$ with $140 \mathrm{mM} \mathrm{KCl)}$ and BSA in PBS: a) DLS analysis of ZSNPS in PBS prepared with $14 \mu \mathrm{M}$ of $C B[8], M V$, and Azo moieties, b) DLS analysis of 5 wt\% BSA in PBS, c) DLS analysis of 1:2 V/V mixture of ZSNP and BSA solutions, d) SEM image of ZSNPS observed in BSA e) SEM image of ZSNPS observed at the drying edge of the SEM sample.

Size tunability of the self-assembled ZSNPs was evaluated in aqueous solution by altering the ratio of the monovalent Azo-Zwit and the multivalent $\mathrm{Azo}_{8}$-PAMAM while keeping the overall CB[8]:MV:Azo stoichiometry at 1:1:1. By increasing the amount of Azo from $\mathrm{Azo}_{8}-$ PAMAM from $10 \%$ to $30 \%$, while decreasing the corresponding amount of Azo-Zwit from $90 \%$ to $70 \%$, an increase in ZSNP size was observed from $51 \pm 11 \mathrm{~nm}$ to $78 \pm 9 \mathrm{~nm}$ by SEM and from $62 \pm 16 \mathrm{~nm}$ to $133 \pm 19 \mathrm{~nm}$ by DLS (see Figures 7.1 and 7.7). This size tuning is attributed to the competition between monovalent and multivalent heteroternary interactions, similar to the SNP formulations stabilized with monovalent PEG reported in Chapter 3 and 5. 

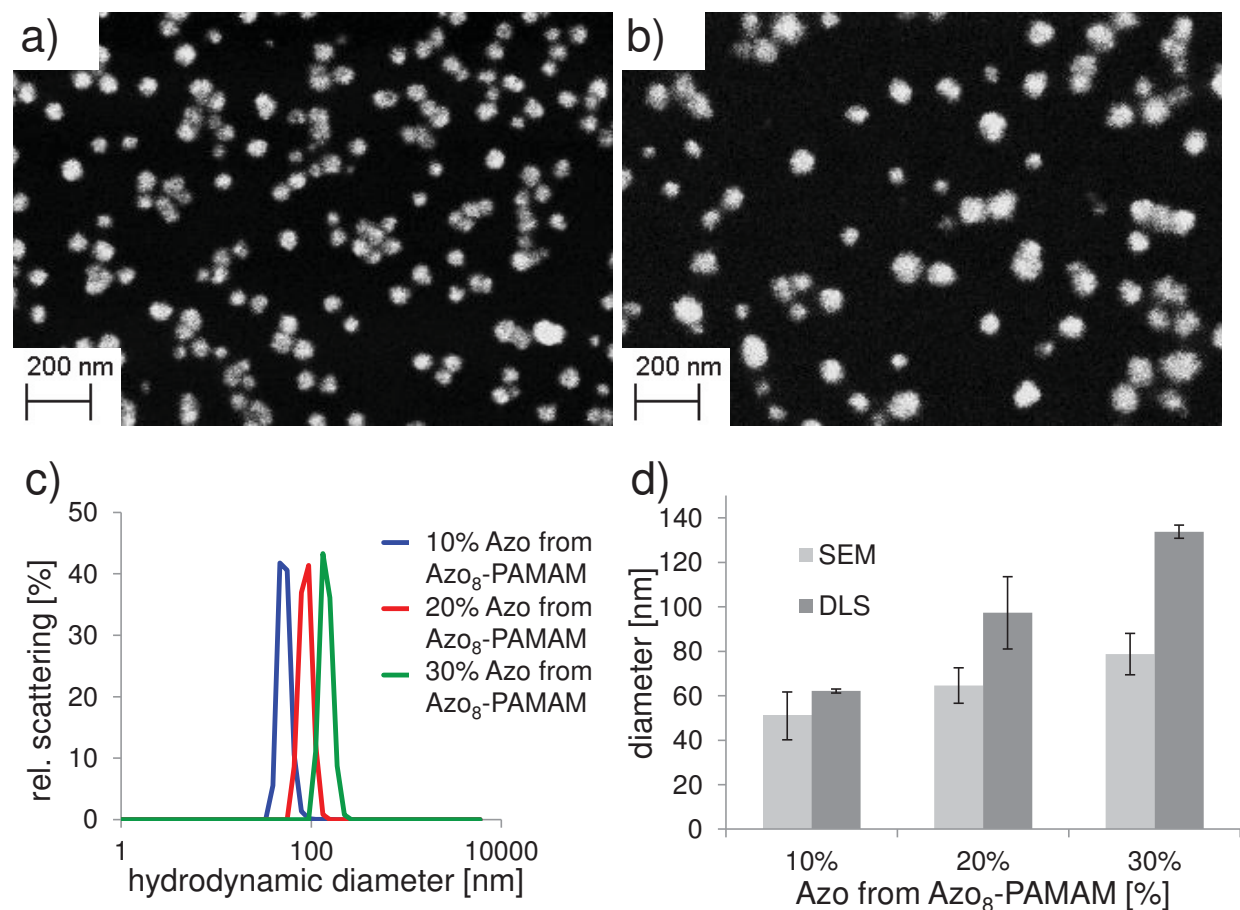

Figure 7.7: Size determination of ZSNPS prepared in water using different stoichiometries of the mono- and multivalent Azo components while maintaining CB[8]:MV:Azo at 1:1:1: a-b) SEM images of ZSNPs prepared in water with a) $10 \%$ and b) $20 \%$ Azo from Azos-PAMAM dendrimer. c) DLS graphs obtained for the ZSNPS prepared with different formulations in water. d) ZSNP diameters measured by SEM ( ) and DLS ( $\square$ ).

\subsubsection{Responsive properties of zwitterionic supramolecular nanoparticles}

The reversibility of the $\mathrm{CB}[8]: M V$ :Azo host-guest complex, induced by photoswitching of the Azo moiety, has been used to trigger NP assembly and disassembly as shown in Chapter 5 and by Scherman and coworkers. ${ }^{25}$ Photoisomerization of trans to cis-Azo by UV light causes disassembly of the ternary complex because the bulkier cis-Azo and MV do not fit in the cavity of $\mathrm{CB}[8]$ simultaneously. To evaluate whether the photoswitching of Azo can be used for the rupture of ZSNPs, the particles in aqueous solution were irradiated with UV light. The effect of UV irradiation on the size of the ZSNPs measured by DLS is shown in Figure 7.8. As observed for SNPs prepared with Azo-PEG ( $\mathrm{M}_{\mathrm{W}} 5 \mathrm{kDa}$ ) in Chapter 5, the ZSNPs started to aggregate after short UV irradiation. This effect continued until no ZSNPs were observed after prolonged UV exposure overnight. The relatively slow particle disassembly process is attributed to incomplete transisomerization (ca. 80\%) and slow rearrangement of multivalently linked building blocks resulting in fast shell but slow core dissolution. In addition, ZSNP re-assembly was achieved upon cis-trans isomerization of the Azo-bearing building blocks by irradiation with visible light for $8 \mathrm{~h}$. 

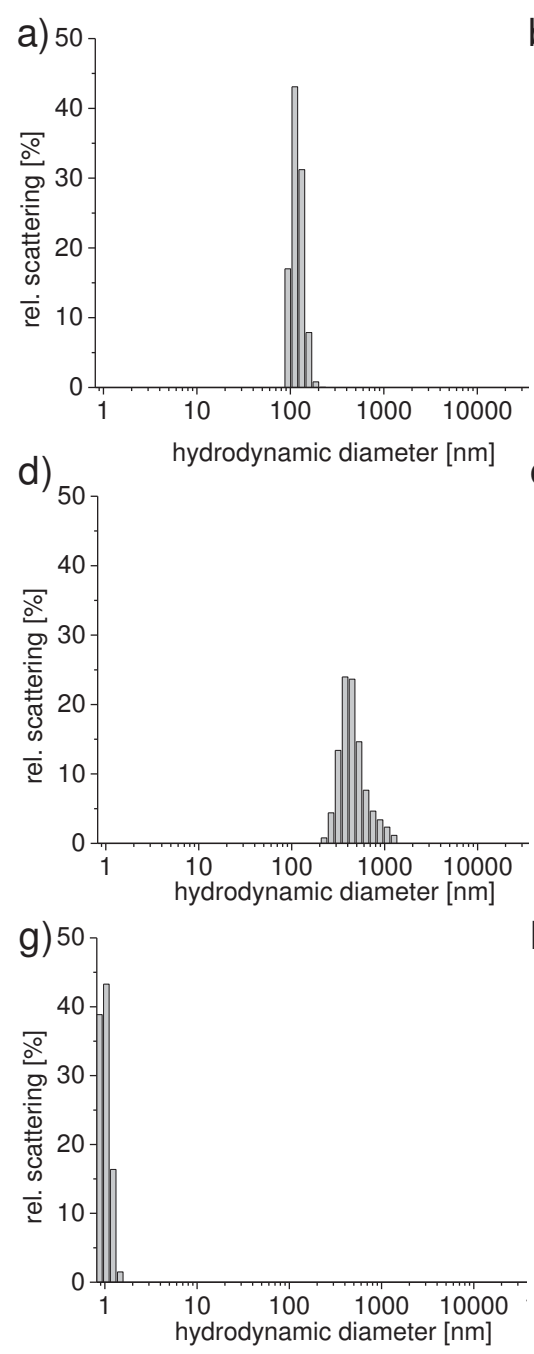
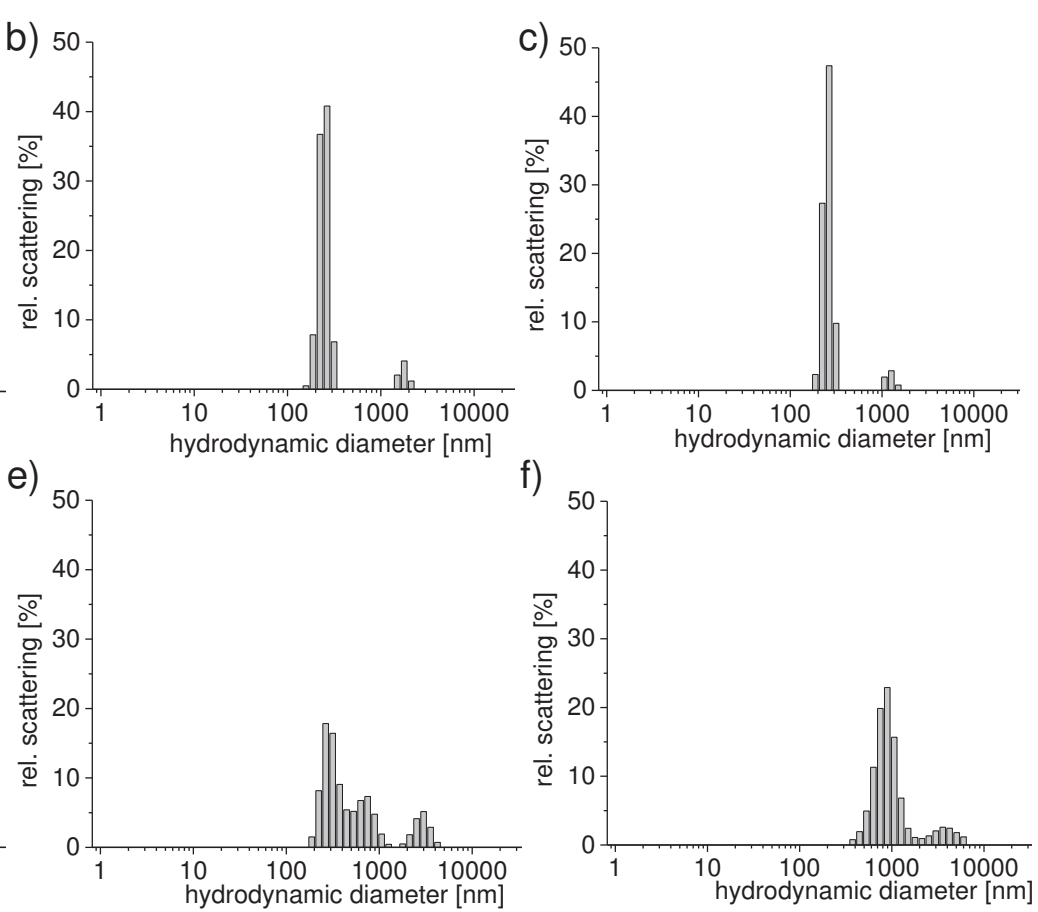

Figure 7.8: DLS analysis of a ZSNP solution: a) as assembled, after UV irradiation for b) $10 \mathrm{~min}, c) 1$ $h, d) 2 h, e) 3.5 h, f) 6 h, g) 14 h$, and h) after subsequent irradiation with visible light for $8 h$.

Whether the cis-Azo or the MV moiety stays in the cavity of the CB[8] after photoswitching, depends on the molecular structure of the components. If the Azo derivative contains a positive charge in close proximity to the Azo moiety, the cis-Azo isomer is stabilized in the $\mathrm{CB}[8]$ cavity by the interaction of the positively charged guest with the ring of carbonyls surrounding the $\mathrm{CB}[8]$ cavity, thus leading to release of MV upon trans-cis isomerization. ${ }^{26}$ In contrast, in the absence of a cationic charge close to the Azo moiety, the MV stays in the $\mathrm{CB}[8]$ cavity and the cis-Azo is expelled. ${ }^{27}$ As the positive charge in Azo-Zwit is not located in the immediate vicinity of the Azo moiety and the charge is partially compensated by the carboxylate anion at the tail of the molecule, it was not clear a priori which moiety would remain bound upon UV-induced trans-cis isomerization. UV/Vis spectroscopy (Figure 7.9) of cis-Azo-Zwit in absence and presence of $\mathrm{CB}[8]$ showed that the cis isomer can be encapsulated in the cavity as witnessed by the decrease of the absorbance of cis-Azo-Zwit at $\lambda=432 \mathrm{~nm}$ characteristic for inclusion in $\mathrm{CB}[8]$. However, in case MV was present in the solution as well, the absorbance 
decrease was only half. Therefore we conclude that an equilibrium between $\mathrm{CB}[8]$-bound MV and cis-Azo-Zwit exists after trans-cis photoswitching.

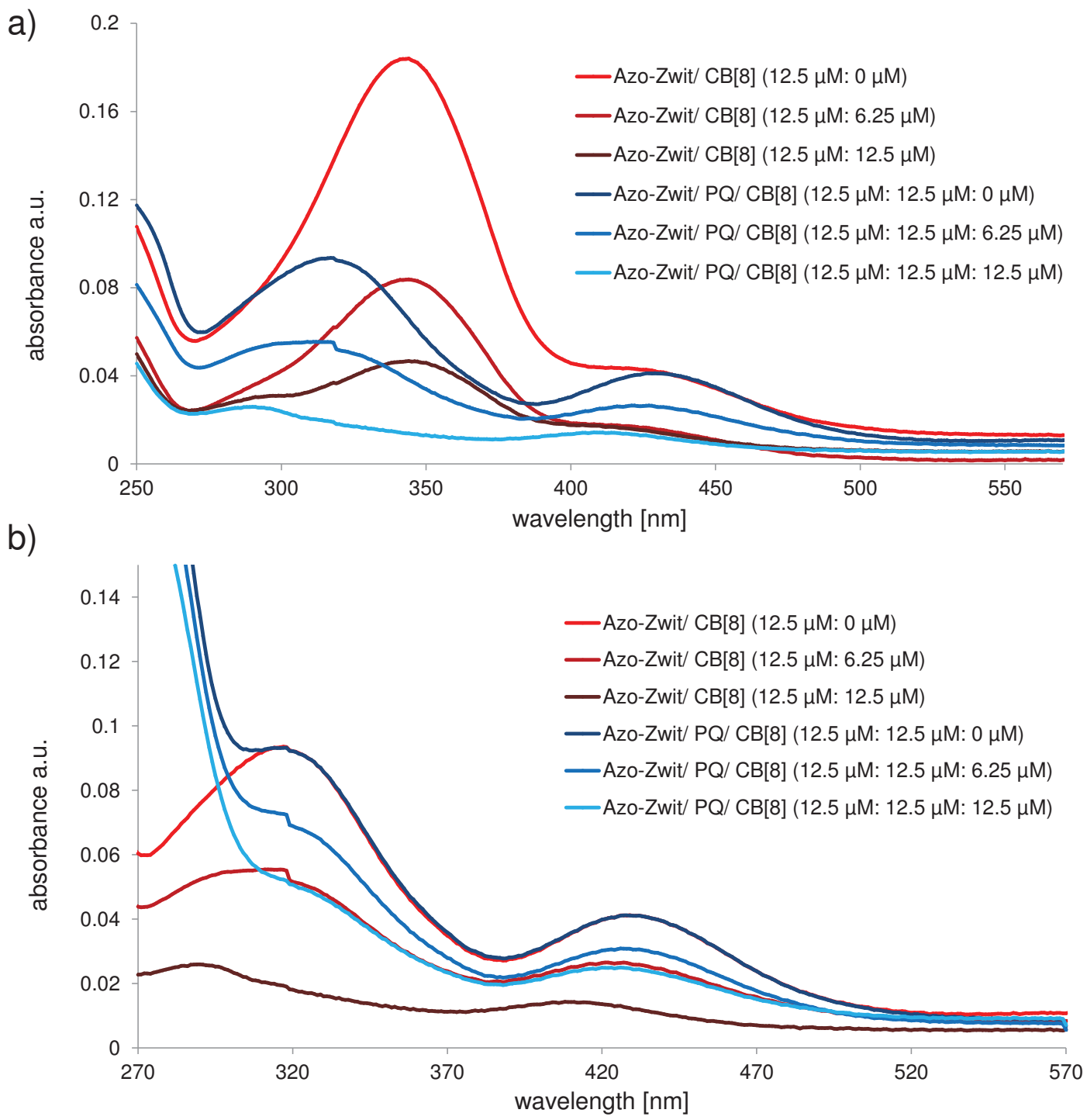

Figure 7.9: UV-Vis absorbance of Azo-Zwit and Azo-Zwit and paraquat (PQ) in the presence of different concentrations of $\mathrm{CB}[8]$ a) under ambient light conditions and b) in dark, after UV irradiation.

To study the behavior of the ZSNPs as a function of $\mathrm{pH}$, the supramolecular building blocks were dissolved in PBS at pH 5.6, 6.2, 6.8 and 7.4 prior to mixing. DLS and SEM images showed that individual ZSNPs were formed at pH 5.6 and 7.4 (see Figures 7.3 and 7.10). In contrast, both techniques indicated the formation and simultaneous aggregation of ZSNPs at the intermediate $\mathrm{pH}$ values 6.2 and 6.8 (Figure 7.10) with the stronger aggregation at $\mathrm{pH}$ 6.8. The aggregates clearly contain individual particles of similar size as formed at $\mathrm{pH} 7.4$, which indicates that the aggregates are hierarchical assemblies of ZSNPs. Notably, the $\mathrm{pH}$ regime of $6.2-6.8$ corresponds with the 
extracellular $\mathrm{pH}$ observed in tumor tissue. Equally important to note, the $\mathrm{pH}$ regime corresponds also with the $\mathrm{p} K_{w}$ (13.7) of the water equilibrium, which indicates a balance between hydronium cations and hydroxide anions at $\mathrm{pH}$ 6.8. A similar aggregation effect in this $\mathrm{pH}$ range was reported by Liu et al. for gold NPs modified with mixed monolayers of 11-mercaptoundecanoic acid and (10-mercaptodecyl)-trimethyl ammonium bromide. ${ }^{28}$ Only when using equimolar fractions of the carboxylic acid and quaternary ammonium adsorbates, was Au NP aggregation observed in a window between $\mathrm{pH} 6.0$ and 7.0, whereas individual Au NPs were observed above and below this regime. We assume that, at all $\mathrm{pH}$ values used here, the ZSNPs are surrounded by overall charge-neutral Azo-Zwit bearing unprotonated carboxylate ions. We therefore attribute aggregation of the ZSNPs to interaction between the shells in a perfectly charge-balanced environment, while a slight imbalance between hydronium and hydroxide ions is apparently sufficient to prevent aggregation. At the same time, we note that this aggregation mechanism may be more universal, and can potentially be used to design self-assembling materials that will show an enhanced EPR effect.
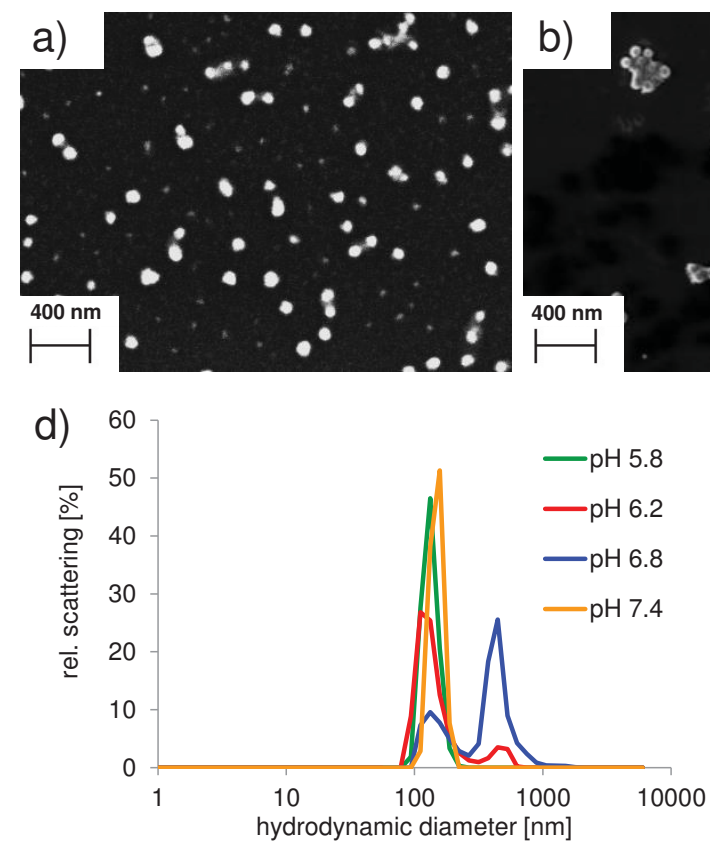
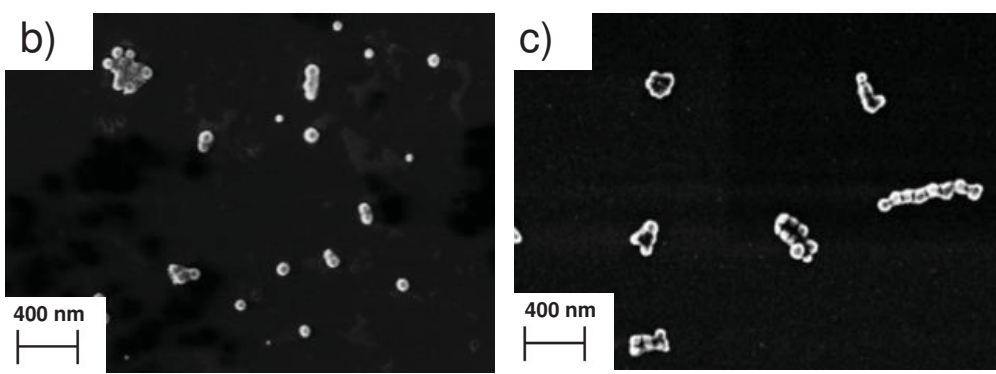

e)

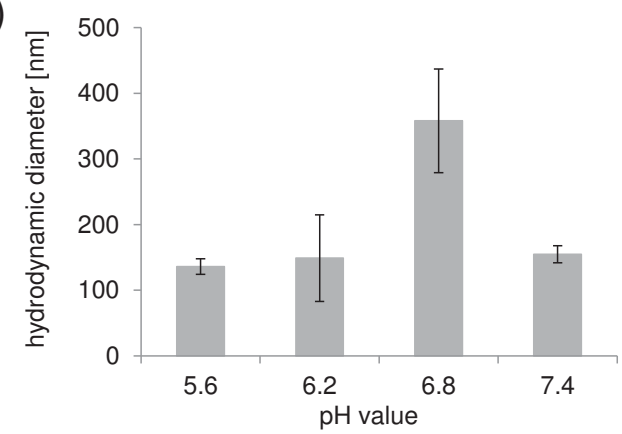

Figure 7.10: SEM images of ZSNPS formed in PBS at: a) $\mathrm{pH} 5.6$, b) $\mathrm{pH} 6.2, \mathrm{c}) \mathrm{pH}$ 6.8. d) DLS graphs obtained for the ZSNPS prepared with in PBS at different $\mathrm{pH}$ values. e) Average ZSNP diameters measured by DLS.

\subsection{Conclusions}

In conclusion, we have shown the formation of SNPs based on the ternary inclusion interaction between $\mathrm{CB}[8], \mathrm{MV}$ and Azo, using the small-molecule zwitterionic Azo-Zwit as the monovalent capping ligand. In contrast to more conventional SNP formulations 
requiring long PEGs for steric stabilization, colloidal stability of the ZSNPs without this steric effect, was observed in different, biologically relevant media. This supramolecular assembly strategy enables size tuning by stoichiometric control of the ratio of multivalent and monovalent Azo components as well as light-triggered SNP disassembly. The ZSNPS show a variety of properties that make them potentially interesting for biomedical applications. Firstly, the use of Azo-Zwit provides non-fouling properties also at high serum protein concentrations. Secondly, ZSNP aggregation is observed at $\mathrm{pH}$ values between 6.2 and 6.8, indicating a potentially enhanced EPR effect in tumor tissue. All these properties combined underline the promise of ZSNPs as nanovectors in cancer therapeutics.

\subsection{Acknowledgements}

Jens Voskuhl is acknowledged for the synthesis of Azo-terminated poly(amino amine) dendrimer generation 1 and Azo-tri(ethylene glycol). Mark Smithers is acknowledged for the SEM images.

\subsection{Experimental Section}

\subsubsection{Materials}

Starting materials for organic synthesis were obtained from Sigma-Aldrich and ABCR and used as received. Methyl-4,4-bipyridinium (methyl viologen, MV)-substituted poly(ethylene imine) (MV-PEI, degree of substitution: 4.5 MV units per polymer chain) and azobenzene (Azo)-terminated poly(amido amine) dendrimer of generation 1 (8 end groups) were synthesized as described in Chapters 3 and 5, respectively. Azobenzenetri(ethylene glycol) was prepared according to the literature procedure. ${ }^{29}$ Cucurbit[8]uril (CB[8]) was purchased from Strem Chemicals and the concentration of a stock solution was assessed by microcalorimetric titration (Microcal VP-ITC) against paraquat. Deuterated solvents used for NMR spectroscopy were purchased from Cambridge Isotope and the water used for dialysis and SNP preparation was of MilliQ quality (Millipore, $R=$

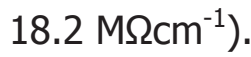

\subsubsection{Synthetic procedures}

\section{$\underline{N}, N$-Dimethyl-3-(4-(phenyldiazenyl)phenoxy)propan-1-amine}<smiles>CN(C)CCCOc1ccc(N=Nc2ccccc2)cc1</smiles>

To a solution of 1-bromo-3-(N,N-diethyl)propane hydrobromide (200 mg, $0.405 \mathrm{mmol}, 1 \mathrm{eq}$ ) in $10 \mathrm{~mL}$ acetonitrile, $\mathrm{K}_{2} \mathrm{CO}_{3}(170 \mathrm{~g}, 0.61 \mathrm{mmol}, 1.5 \mathrm{eq})$ was added, and the suspension was stirred for $30 \mathrm{~min}$ at $70^{\circ} \mathrm{C}$. After that time 4-phenylazophenol (160 mg, $0.405 \mathrm{mmol}, 1 \mathrm{eq}$ ) was added an stirring was 
continued at $80{ }^{\circ} \mathrm{C}$ for $12 \mathrm{~h}$, followed by removal of the solvent. The residue was dissolved in $25 \mathrm{~mL}$ of $\mathrm{CHCl}_{3}$ and extracted three times with distilled water $(10 \mathrm{~mL})$. The organic layer was separated and dried over $\mathrm{MgSO}_{4}$. The solvent was evaporated and the residue was subjected to silica gel column chromatography $\left(\mathrm{CH}_{2} \mathrm{Cl}_{2} / \mathrm{MeOH}\right.$ gradient elution 100:1 to 98:2). The product 1 was obtained as a yellow solid. Yield: $143 \mathrm{mg}$, $62 \%$. ${ }^{1} \mathrm{H}-\mathrm{NMR}\left(400 \mathrm{MHz}, \mathrm{CDCl}_{3}\right): \delta=7.86-7.79(\mathrm{~m}, 4 \mathrm{H}, 4 \mathrm{CH}) ; 7.45-7.41(\mathrm{~m}, 2 \mathrm{H}, 2 \mathrm{CH})$; 7.39-7.35 (m, $1 \mathrm{H}, \mathrm{CH}) ; 6.96-6.92(\mathrm{~m}, 2 \mathrm{H}, 2 \mathrm{CH}) ; 4.07-4.04\left(\mathrm{~m}, 2 \mathrm{H}, \mathrm{CH}_{2}\right) ; 2.56(\mathrm{t}, 2 \mathrm{H}$, $\left.\mathrm{CH}_{2}\right) ; 2.32-2.27\left(\mathrm{~s}, 6 \mathrm{H}, 2 \mathrm{CH}_{3}\right) ; 2.05-1.99\left(\mathrm{q}, 2 \mathrm{H}, \mathrm{CH}_{2}\right) .{ }^{13} \mathrm{C}-\mathrm{NMR}\left(100 \mathrm{MHz}, \mathrm{CD}_{2} \mathrm{Cl}_{2}\right): \delta=$ $163.5,154.6,148.8,132.3,130.9,126.5,124.3,116.6,68.2,57.8,46.8,31.6,28.8$. ESIMS (m/z): calculated for $\left[\mathrm{C}_{17} \mathrm{H}_{21} \mathrm{~N}_{3} \mathrm{OH}\right]^{+}: 284.17$, found: 284.20 .

3-(Dimethyl-(3-(4-phenyldiazenyl)phenoxy)propyl)ammonio)propanoate (Azo-Zwit)

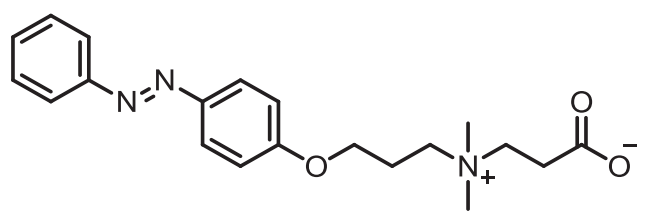

To an ice-cooled solution of $1(60 \mathrm{mg}, 0.21 \mathrm{mmol}$ $1 \mathrm{eq}$ ) in $10 \mathrm{~mL}$ dry acetone, $\beta$-propiolactone (18 $\mathrm{mg}, 0.252 \mathrm{mmol}, 1.2 \mathrm{eq}$ ) in $1 \mathrm{~mL}$ acetone was added slowly. The reaction mixture was stirred 2 $\mathrm{h}$ at $5^{\circ} \mathrm{C}$ and slowly warmed up to RT. The yellow precipitate was filtered and washed with anhydrous acetone and anhydrous ether. The product was dried under reduced pressure to obtain the final (E)-3-(dimethyl-(3-(4phenyldiazenyl)phenoxy)propyl)ammonio) propanoate (Azo-Zwit). Yield: $27 \mathrm{mg}, 36 \% .{ }^{1} \mathrm{H}-$ NMR (400 MHz, $\left.\mathrm{D}_{2} \mathrm{O}\right): \delta=7.75-7.69$ (dd, 4H, 4CH); 7.50-7.48 (d, 3H, 3CH); 7.03-7.01 $(\mathrm{d}, 2 \mathrm{H}, 2 \mathrm{CH}) ; 4.11\left(\mathrm{t}, 2 \mathrm{H}, \mathrm{CH}_{2}\right) ; 3.54-3.50\left(\mathrm{t}, 2 \mathrm{H}, \mathrm{CH}_{2}\right) ; 3.43\left(\mathrm{t}, 2 \mathrm{H}, \mathrm{CH}_{2}\right) ; 3.02(\mathrm{~s}, 6 \mathrm{H}$, $\left.2 \mathrm{CH}_{3}\right) ; 2.70-2.66\left(\mathrm{t}, 2 \mathrm{H}, \mathrm{CH}_{2}\right) ; 2.18-2.12\left(\mathrm{t}, 2 \mathrm{H}, \mathrm{CH}_{2}\right) .{ }^{13} \mathrm{C}-\mathrm{NMR}\left(150 \mathrm{MHz}, \mathrm{DMSO}-\mathrm{d}_{6}\right): \delta=$ $170.98,161.43,152.46,146.95,131.40,129.89,129.57,125.08,123.44,122.74$, 119.78, 115.63, 114.88, 65.69, 61.25, 60.81, 50.64, 30.28, 20.68. ESI-MS (m/z): calculated for $\left[\mathrm{C}_{20} \mathrm{H}_{25} \mathrm{~N}_{3} \mathrm{O}_{3} \mathrm{H}\right]^{+}:: 356.19$, found: 356.19 .

\subsubsection{Methods}

Zwitterionic supramolecular nanoparticle assembly

For the preparation of the zwitterionic supramolecular nanoparticles (ZSNPs), aqueous or PBS solutions of $\mathrm{CB}[8], \mathrm{MV}-\mathrm{PEI}$ and different concentrations of Azo-Zwit were prepared before mixing. DMSO was used to dissolve different concentrations of Azo8-PAMAM. For example, for preparing a solution having 30\% Azo entities derived from the aliphatic $\mathrm{AzO}_{8}$-PAMAM dendrimer, $1000 \mu \mathrm{L}$ MV-PEI $(0.31 \mu \mathrm{M})$ was added to a previously prepared solution of $500 \mu \mathrm{L}$ Azo-Zwit $(1.96 \mu \mathrm{M}), 20 \mu \mathrm{L}$ Azo ${ }_{8}$-PAMAM $(2.65 \mu \mathrm{M})$ and $500 \mu \mathrm{L}$ CB[8] $(2.8 \mu \mathrm{M})$. The sample was mixed and kept at room temperature for 2 days before DLS and SEM analysis. 
Control experiments for zwitterionic supramolecular nanoparticle formation

To evaluate whether ZSNP formation is triggered by the formation of the ternary complex between $\mathrm{CB}[8], \mathrm{MV}$ and Azo, different control experiments were carried out. ZSNP formation using $30 \%$ Azo from $\mathrm{AzO}_{8}$-PAMAM was carried out without $\mathrm{CB}[8]$, without MVPEI or in the presence of of the cucurbit[n]uril homolog $\mathrm{CB}[7]$. Hereto, the ZSNP solutions were prepared with $500 \mu \mathrm{L}$ water or $500 \mu \mathrm{L} \mathrm{CB}$ [7] $(2.8 \mu \mathrm{M})$ instead of $500 \mu \mathrm{L}$ $\mathrm{CB}[8](2.8 \mu \mathrm{M})$. Additionally, ZSNP formation was evaluated in the absence of MV-PEI.

Supramolecular nanoparticle formation with Azo-tri(ethylene glycol)

To get a better understanding of the stabilizing role of Azo-Zwit in the formation of ZSNPs, SNP assembly was attempted in PBS using Azo-tri(ethylene glycol) as the shellforming component. Therefore different formulations, containing $10 \%, 20 \%$ and $30 \%$ Azo from $\mathrm{AzO}_{8}$-PAMAM, were tested according to the procedure described for ZSNP preparation and the formation of SNPs reported in Chapter 5.

\section{Formation and stability of ZSNPs in PBS with different salt concentrations}

In order to investigate the ZSNP formation in PBS at different ionic strengths, solutions of MV-PEI $(0.31 \mu \mathrm{M})$, Azo-Zwit $(1.96 \mu \mathrm{M})$ and $\mathrm{CB}[8](2.8 \mu \mathrm{M})$ were prepared in PBS $(\mathrm{pH} 7.4)$ containing $50 \mathrm{mM}, 100 \mathrm{mM}, 140 \mathrm{mM}, 300 \mathrm{mM}$ and $700 \mathrm{mM} \mathrm{KCl}$. These solutions were mixed according the recipe given for the preparation of the ZSNPs in water (containing $30 \%$ Azo from $\mathrm{AzO}_{8}$-PAMAM). ZSNP formation was analyzed by DLS after 2 days at RT and by SEM 7 days after preparation.

Stability of ZSNPs in PBS containing BSA to imitate protein concentration in human body To verify the stability of the ZSNPs in the presence of proteins, ZSNPs were formed in PBS using an overall concentration of $14 \mu \mathrm{M}$ of the supramolecular host-guest binding partners and $30 \%$ Azo from Azo $_{8}$-PAMAM. This mixture was kept $2 \mathrm{~d}$ at room temperature to ensure stable ZSNP formation. In addition, a $5 \mathrm{wt} \%$ dispersion of BSA in water was prepared. Stability of the ZSNPs was controlled after mixing $500 \mu \mathrm{L}$ of the BSA solution with $250 \mu \mathrm{L}$ of the ZSNP solution. DLS analysis was carried out for the ZSNPs, BSA and a mixture of both. Additionally SEM was carried out 1d after mixing the two solutions.

\section{Light response of supramolecular nanoparticles}

To evaluate the photoresponsive properties of the particles, ZSNPs containing 30\% Azo from $\mathrm{Azo}_{8}$-PAMAM were prepared and analyzed. Samples were irradiated with UV light at $\lambda<400 \mathrm{~nm}$ for different times. DLS analysis was performed immediately after exposure, while keeping the SNP samples in the dark. Representative DLS scattering graphs are given in Figure 7.8, although the polydispersity of the irradiated samples were very high and these are not absolute values. 


\section{pH-induced aggregation of the ZSNPs}

In order to investigate the ZSNP formation in PBS, solutions of MV-PEI $(0.31 \mu \mathrm{M})$, AzoZwit $(1.96 \mu \mathrm{M})$ and $\mathrm{CB}[8](2.8 \mu \mathrm{M})$ were prepared in PBS at $\mathrm{pH}$ 5.6, 6.2, 6.8 and 7.4. ZSNP formation (30\% Azo from $\mathrm{AzO}_{8}$-PAMAM) at these $\mathrm{pH}$ values was carried out as described for ZSNP preparation in water by mixing the aqueous solutions with $\mathrm{AzO}_{8}{ }^{-}$ PAMAM in DMSO $(2.62 \mu \mathrm{M})$. ZSNP formation was analyzed by DLS after 2 days at RT and by SEM 5 days after preparation.

\subsubsection{Equipment}

\section{Dynamic light scattering (DLS)}

DLS experiments were performed with a Nanotrac by Anaspec operating with a Microtrac FLEX Operating Software at $25^{\circ} \mathrm{C}$ using a laser wavelength of $780 \mathrm{~nm}$ at a scattering angle of $90^{\circ}$. The observed sizes and standard derivations of the ZSNPs were calculated by taking an average of 5 measurements. In total, particles with sizes ranging from 0.8 to $6400 \mathrm{~nm}$ were detected. The DLS curves shown in this chapter are representative for the individual measurements.

\section{Scanning electron microscopy (SEM)}

All SEM images were taken with a 1500 high resolution scanning electron microscope. Therefore the aqueous SNP solutions were drop-cast on a Formvar coated copper TEM grid. The solution was dried and analyzed without further treatment of the samples.

\section{UV irradiation}

UV light irradiation experiments were carried out with a Hönle bluepoint 2 easycure using a shortpass filter $\lambda \leq 400 \mathrm{~nm}$. After formation, the SNPs were treated with UV light for different periods. Therefore the samples were filled in a quartz cuvette and exposed to light with $\lambda \leq 400 \mathrm{~nm}$. To analyze the ongoing disassembly process, the samples were analyzed by DLS in dark and UV irradiation was continued.

To evaluate the SNP re-assembly, the SNP solution was kept at visible light for $8 \mathrm{~h}$ and analyzed afterwards.

\section{$\underline{\text { UV/Vis spectroscopy }}$}

UV/Vis absorption spectra were recorded using a Perkin Elmer Lambda 850 UV-Vis spectrometer. Supramolecular encapsulation of Azo-Zwit in presence of $\mathrm{CB}[8]$ and in presence of paraquat (MV) and $\mathrm{CB}[8]$ was evaluated by UV/Vis spectroscopy at room temperature. Stock solutions of Azo-Zwit, $\mathrm{CB}[8]$ and paraquat were prepared in water. The stock solutions were mixed to result in solutions with Azo-Zwit (12.5 $\mu \mathrm{M})$, AzoZwit/paraquat (12.5 $\mu \mathrm{M} / 12.5 \mu \mathrm{M})$, Azo-Zwit/CB[8] (12.5 $\mu \mathrm{M} / 6.25 \mu \mathrm{M})$, AzoZwit/paraquat/CB[8] (12.5 $\mu \mathrm{M} / 12.5 \mu \mathrm{M} / 6.25 \mu \mathrm{M})$, Azo-Zwit/CB[8] $(12.5 \mu \mathrm{M} / 12.5 \mu \mathrm{M})$, Azo-Zwit/paraquat/CB[8] $(12.5 \mu \mathrm{M} / 12.5 \mu \mathrm{M} / 12.5 \mu \mathrm{M})$. The UV/Vis absorbance spectra of 
these solutions were measured at visible light conditions and after irradiation with UV light to evaluate the encapsulation efficiencies of the two photoisomers of Azo-Zwit by $\mathrm{CB}[8]$ in the absence and presence of paraquat.

\section{NMR spectroscopy}

${ }^{1} \mathrm{H}$ and ${ }^{13} \mathrm{C}$ NMR spectra were recorded on Bruker $400 \mathrm{MHz}$ and $600 \mathrm{MHz}$ spectrometers. ${ }^{1} \mathrm{H}$ and ${ }^{13} \mathrm{C}$ chemical shift values, measured at $400 \mathrm{MHz}$ and $150 \mathrm{MHz}$, respectively, are reported as $\delta$ using the residual solvent signal as internal standard.

\section{Mass spectrometry}

Mass analysis was performed by electrospray ionization using micromass LCT from Waters/Micromass.

\subsection{References}

1. C.-Y. Lai, B. G. Trewyn, D. M. Jeftinija, K. Jeftinija, S. Xu, S. Jeftinija and V. S. Y. Lin, Journal of the American Chemical Society, 2003, 125, 4451-4459.

2. P. Huang, D. Wang, Y. Su, W. Huang, Y. Zhou, D. Cui, X. Zhu and D. Yan, Journal of the American Chemical Society, 2014, 136, 11748-11756.

3. L. Brannon-Peppas and J. O. Blanchette, Advanced Drug Delivery Reviews, 2012, 64, Supplement, 206-212.

4. B. E. Rolfe, I. Blakey, O. Squires, H. Peng, N. R. B. Boase, C. Alexander, P. G. Parsons, G. M. Boyle, A. K. Whittaker and K. J. Thurecht, Journal of the American Chemical Society, 2014, 136, 2413-2419.

5. S. M. Janib, A. S. Moses and J. A. MacKay, Advanced Drug Delivery Reviews, 2010, 62, 1052-1063.

6. J. Nam, N. Won, H. Jin, H. Chung and S. Kim, Journal of the American Chemical Society, 2009, 131, 13639-13645.

7. M. A. Dobrovolskaia, P. Aggarwal, J. B. Hall and S. E. McNeil, Molecular Pharmaceutics, 2008, 5, 487-495.

8. S.-D. Li and L. Huang, Molecular Pharmaceutics, 2008, 5, 496-504.

9. R. Gref, M. Lück, P. Quellec, M. Marchand, E. Dellacherie, S. Harnisch, T. Blunk and R. H. Müller, Colloids and Surfaces B: Biointerfaces, 2000, 18, 301-313.

10. D. E. Owens Iii and N. A. Peppas, International Journal of Pharmaceutics, 2006, 307, 93102.

11. E. Ostuni, R. G. Chapman, R. E. Holmlin, S. Takayama and G. M. Whitesides, Langmuir, 2001, 17, 5605-5620.

12. L. Li, S. Chen and S. Jiang, Journal of Biomaterials Science, Polymer Edition, 2007, 18, 1415-1427.

13. L. Xu, P. Ma, B. Yuan, Q. Chen, S. Lin, X. Chen, Z. Hua and J. Shen, RSC Advances, 2014, 4, 15030-15035.

14. R. S. Smith, Z. Zhang, M. Bouchard, J. Li, H. S. Lapp, G. R. Brotske, D. L. Lucchino, D. Weaver, L. A. Roth, A. Coury, J. Biggerstaff, S. Sukavaneshvar, R. Langer and C. Loose, Science Translational Medicine, 2012, 4, 153 ra132. 
15. K. Glasmästar, C. Larsson, F. Höök and B. Kasemo, Journal of Colloid and Interface Science, 2002, 246, 40-47.

16. Z. Cao, Q. Yu, H. Xue, G. Cheng and S. Jiang, Angewandte Chemie International Edition, 2010, 49, 3771-3776.

17. L. Zhang, H. Xue, C. Gao, L. Carr, J. Wang, B. Chu and S. Jiang, Biomaterials, 2010, 31, 6582-6588.

18. Y. Zhu, H. S. Sundaram, S. Liu, L. Zhang, X. Xu, Q. Yu, J. Xu and S. Jiang, Biomacromolecules, 2014, 15, 1845-1851.

19. S. Eliasof, D. Lazarus, C. G. Peters, R. I. Case, R. O. Cole, J. Hwang, T. Schluep, J. Chao, J. Lin and Y. Yen, Proceedings of the National Academy of Sciences, 2013, 110, 1512715132.

20. H. Fan, Q.-D. Hu, F.-J. Xu, W.-Q. Liang, G.-P. Tang and W.-T. Yang, Biomaterials, 2012, 33, 1428-1436.

21. M. E. Davis, J. E. Zuckerman, C. H. J. Choi, D. Seligson, A. Tolcher, C. A. Alabi, Y. Yen, J.

D. Heidel and A. Ribas, Nature, 2010, 464, 1067-1070.

22. H. Wang, S. Wang, H. Su, K.-J. Chen, A. L. Armijo, W.-Y. Lin, Y. Wang, J. Sun, K.-i. Kamei, J. Czernin, C. G. Radu and H.-R. Tseng, Angewandte Chemie International Edition, 2009, 48, 4344-4348.

23. C. Stoffelen and J. Huskens, Chemical Communications, 2013, 49, 6740-6742.

24. L. Grana Suarez, W. Verboom and J. Huskens, Chemical Communications, 2014, 50, 7280-7282.

25. Y. Lan, Y. Wu, A. Karas and O. A. Scherman, Angewandte Chemie International Edition, 2014, 53, 2166-2169.

26. J. del Barrio, P. N. Horton, D. Lairez, G. O. Lloyd, C. Toprakcioglu and O. A. Scherman, Journal of the American Chemical Society, 2013, 135, 11760-11763.

27. F. Tian, D. Jiao, F. Biedermann and O. A. Scherman, Nature Communications, 2012, 3, 1207.

28. X. Liu, Y. Chen, H. Li, N. Huang, Q. Jin, K. Ren and J. Ji, ACS Nano, 2013, 7, 6244-6257.

29. S. K. M. Nalluri, J. Voskuhl, J. B. Bultema, E. J. Boekema and B. J. Ravoo, Angewandte Chemie International Edition, 2011, 50, 9747-9751. 


\section{Chapter 8}

\section{Self-Assembly of Size-Tunable Supramolecular Nanoparticle Clusters in a Microfluidic Channel}

Clusters of nanoparticles, also called network aggregates, form an important class of materials in the development of multifunctional materials using a toolbox approach. Microfluidics has emerged as a powerful technology in various fields such as chemical synthesis, medical diagnostics and high-throughput biological assays. Only few studies have described the merging of microfluidic technology with supramolecular host-guest chemistry for the fabrication of large self-assembled structures. In this chapter, supramolecular nanoparticle clusters (SNPCS) have been formed in a microfluidic device by controlling the diffusive mixing of the constituting supramolecular building blocks. Cluster formation between ligand-functionalized silica nanoparticles, dendrimers, and poly(ethylene glycol) (PEG) stopper molecules is induced by the ternary charge-transfer complex formation between cucurbit[8]uril, methyl viologen and naphthol. The resulting SNPC size depends strongly on the stoichiometry of the host and guest binding partners, the competition between multivalent and monovalent naphthol entities, and the microfluidic flow conditions. Variation of the PEG length leads to modulation of its diffusion rate and thus to an additional kinetic control parameter of the SNPC formation process. 


\subsection{Introduction}

Self-assembly is the process by which individual components organize into ordered structures spontaneously, and it is a central theme of nanoscience and nanotechnology. ${ }^{1,2}$ Self-assembly enables the formation of functional materials in nano- or micrometer dimensions, based exclusively on non-covalent forces such as Van der Waals interactions, hydrogen bonding, electrostatic interactions, magnetic interactions and coordination chemistry. ${ }^{3,4}$ Supramolecular host-guest chemistry offers the ability to engineer desired structures based on a blend of different non-covalent interactions, which thus enables the formation of stable, yet reversible constructs. Inclusion complexes formed by the interaction of cucurbit[8]uril or cucurbit[10]uril with appropriately sized guest molecules are known to lead to high binding affinities, and their size allows the inclusion of two guests leading to ternary complexes. ${ }^{5}$ Besides supramolecular binding motifs, polymers such as poly(ethylene glycol) (PEG) can be used as structure-directing agents that support the formation of self-assembled functional materials. ${ }^{6}$ Using guest-modified PEG, we have demonstrated the formation of size-tunable (Chapter 3 ) and dual responsive (Chapter 8) supramolecular nanoparticles (SNPs) mediated by the heteroternary interaction of $\mathrm{CB}[8]$, methyl viologen (MV), and naphthol (Np), or of $\mathrm{CB}[8]$, MV, and azobenzene, respectively.

The modification of inorganic nanoparticles with host and guest moieties has been an established strategy to fabricate nanoparticle networks in aqueous solution. ${ }^{7-9}$ For example, the clustering of $\beta$-cyclodextrin-functionalized silver nanoparticles with aromatic guest molecules provides a sensitive detection method of different isomers by eye. ${ }^{10}$ The optical, magnetic and biological properties of clustered materials have been shown to depend strongly on their size and conformation. ${ }^{11}$

The ultimate goal of chemists is to control the properties of a structure prior to synthesis. This requires superior control over the fabrication of the resulting structures, especially in self-assembly processes, because the formation of supramolecular host-guest structures is strongly dependent on thermodynamic and kinetic parameters. We have recently shown that the assembly kinetics of adamantane/cyclodextrin nanoparticle network aggregates can be varied by the flow conditions in a multi-inlet vortex mixer. ${ }^{12}$ In contrast to the turbulent flow conditions in such a mixer, uniform and well-defined laminar flow is observed within microfluidic reactors.

Microfluidics deals with the manipulation of small volumes of liquids in a microscale channel, and its large applicability and versatility have allowed it to grow into a powerful interdisciplinary technology. ${ }^{13}$ The micrometer device dimensions provide a large surfaceto-volume ratio, rapid heat transfer, low Reynolds numbers and unique mass transport properties which are beneficial in applications such as chemical synthesis, ${ }^{14}$ medical 
diagnostics, ${ }^{15}$ high throughput biological assays, ${ }^{16}$ controlled synthesis of nano-materials ${ }^{17}$ and the controlled self-assembly of various nanostructures. ${ }^{18-24}$ In microfluidic devices, liquid streams from different inlets flow in parallel, and the mixing of the interacting components in those streams is driven by lateral molecular diffusion. This diffusive mixing is strongly dependent on the diffusion properties of the building blocks and the residence time in the microfluidic device, and can therefore be directly regulated by the flow rates of the ingoing fluid streams. For electrostatic interactions, the formation of soft NPs was controlled using hydrodynamic flow focusing, by tuning the residence time, temperature and pressure. ${ }^{25,26}$ The assembly of gold and iron oxide nanoparticles onto silica nanoparticles has been controlled by varying the flow rates within a multistep microfluidic device. ${ }^{27}$ The self-assembly of porphyrin architectures has been shown recently to be regulated by the flow field in a micro-flow environment. ${ }^{28}$

Only few studies have been described in literature in which microfluidic technology is merged with the field of supramolecular host-guest chemistry for the fabrication of large self-assembled structures. Tseng and co-workers have used a digital microreactor to prepare an assortment of cell-targeting and DNA encapsulated SNPs based on the interaction of $\beta$-cyclodextrin and adamantane, providing a better dispersity and higher reproducibility than in batch processes. ${ }^{29,30}$ Using the same host-guest chemistry, Thompson and co-workers have described the variation of polymeric supramolecular nanoparticle properties (diameter, polydispersity and zeta-potential) by controlling the velocity of the two mixing inlet streams within a microfluidic reactor. ${ }^{31}$ The groups of Scherman and Abell have shown the formation of monodisperse supramolecular microcapsules self-assembled within microfluidic droplets using the ternary interaction between $\mathrm{CB}[8]$, and $\mathrm{MV}$ and $\mathrm{Np}$ building blocks. ${ }^{32}$ Nevertheless, the diffusive mixing omnipresent in microfluidics has not been used so far to control the formation of hostguest nanoparticle network aggregates.

In this chapter, we present the size-controlled self-assembly of aggregates of nanoparticles, so-called supramolecular nanoparticle clusters (SNPCs) (Scheme 8.1), mediated by multiple heteroternary host-guest interactions of $\mathrm{CB}[8]$, MV and Np. Cluster formation is induced by multivalent interactions forming between the MV-modified silica nanoparticles $\left(\mathrm{SiO}_{2}-\mathrm{MV}\right)$, a multivalent $\mathrm{Np}$-modified dendrimer, and $\mathrm{CB}[8]$, whereas $\mathrm{Np}$ PEG terminates the aggregation by monovalent interaction at the surrounding cluster shell. Aggregate formation was carried out comparing mixing in batch conditions and diffusive mixing within a microfluidic device. Only under diffusive mixing conditions, controlled SNCP formation and size tunability was observed by competition between the mono- and multivalent Np-bearing components, by changing the mixing time of the interacting host and guest binding partners in the microreactor. 
a)

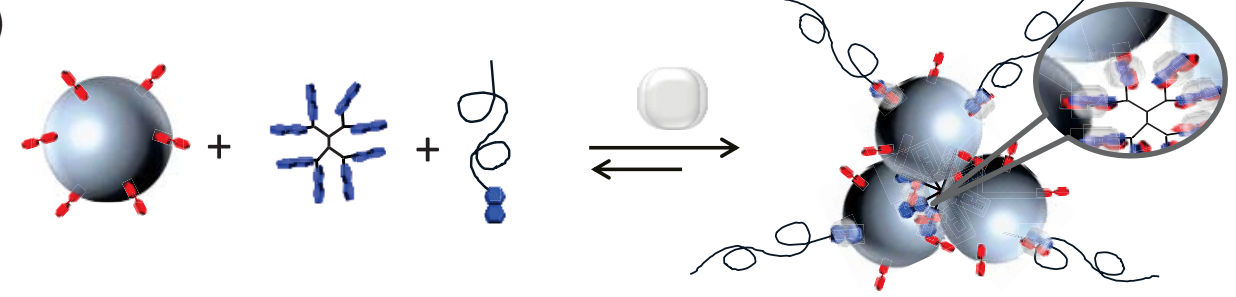

b)

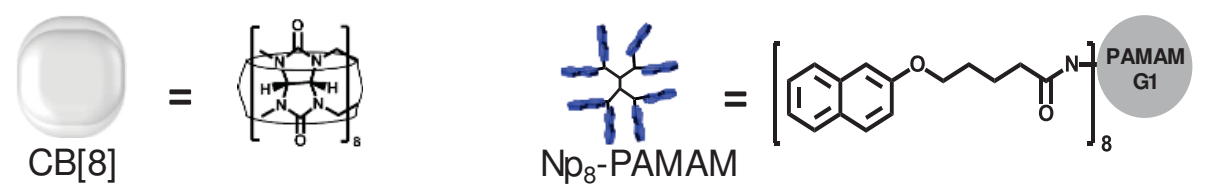

c)
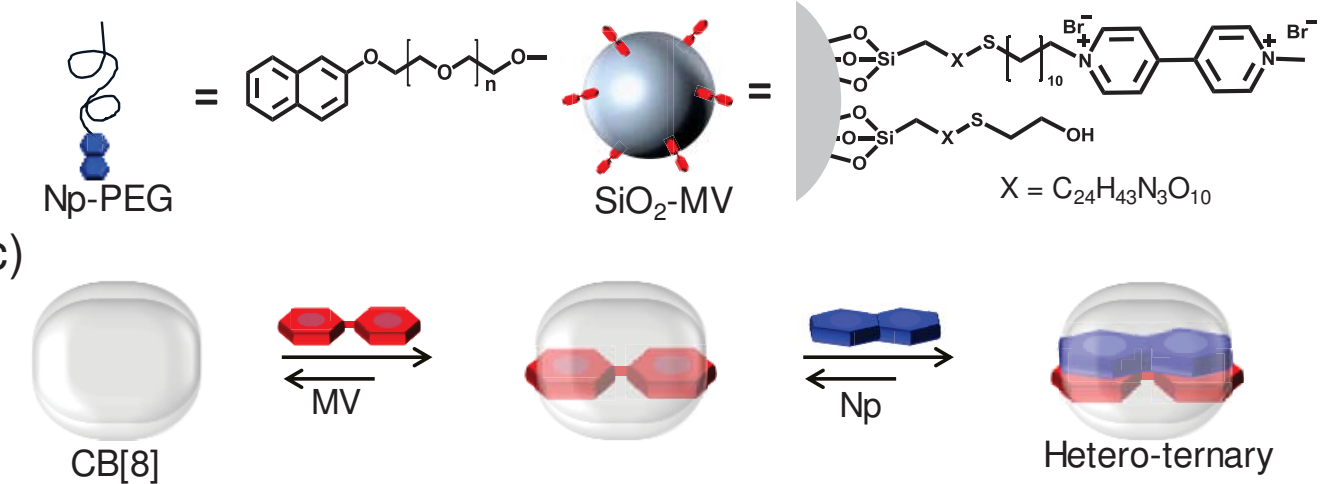

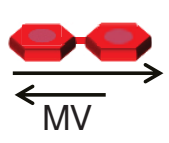

$\mathrm{CB}[8]$
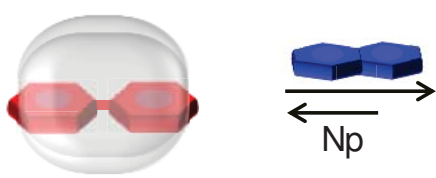

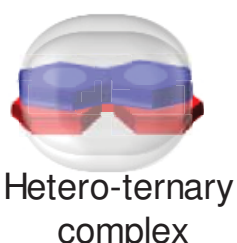

Scheme 8.1: a) Schematic illustration of supramolecular network aggregation of silica nanoparticles, dendrimers and PEG stopper molecules, mediated by the ternary complex formation between MV, $N p$, and CB[8]. b) Supramolecular building blocks involved in SNPC formation: CB[8], Np-PEG (Mw 1000 or $5000 \mathrm{~g} / \mathrm{mol}), \mathrm{Np}_{8}-\mathrm{PAMAM}$, and $\mathrm{SiO}_{2}-\mathrm{MV}$. c) Ternary complex formation by inclusion of MV in $C B[8]$, followed by inclusion of $N p$.

\subsection{Results and Discussion}

\subsubsection{Supramolecular building blocks for SNPC formation}

As interacting guest-modified building blocks, we used silica nanoparticles functionalized with $\mathrm{MV}\left(\mathrm{SiO}_{2}-\mathrm{MV}\right)$, Np-terminated poly(amido amine) $\mathrm{G} 1$ dendrimer ( $\left.\mathrm{Np}_{8}-\mathrm{PAMAM}\right)$, and Np-terminated poly(ethylene glycol) monomethyl ether (Np-PEG) (Scheme 8.1b). The Npbearing building blocks, Np-PEG $\left(\mathrm{M}_{\mathrm{w}}=5000 / 1000 \mathrm{~g} / \mathrm{mol}\right)$ and $\mathrm{Np}_{8}$-PAMAM, were prepared as described in Chapters 3 and 4 . Silica nanoparticles functionalized with methyl viologen $\left(\mathrm{SiO}_{2}-\mathrm{MV}\right)$ were synthesized by adapting a literature procedure for the functionalization of transferrin-decorated NPs (Scheme 8.2). ${ }^{33}$ Bare silica $\mathrm{NPs}^{34}$ were aminated using 3-(aminopropyl)-triethoxysilane (APTES), followed by reaction with an excess of bifunctional succinimide-maleimide hexa(ethylene glycol) linker. The terminal maleimide group was used to functionalize the particles with 1-(1-undecyl-11-thiol)-1methyl-4,4-bipyridinium, ${ }^{35}$ followed by backfilling the unreacted maleimide groups with mercaptoethanol. 

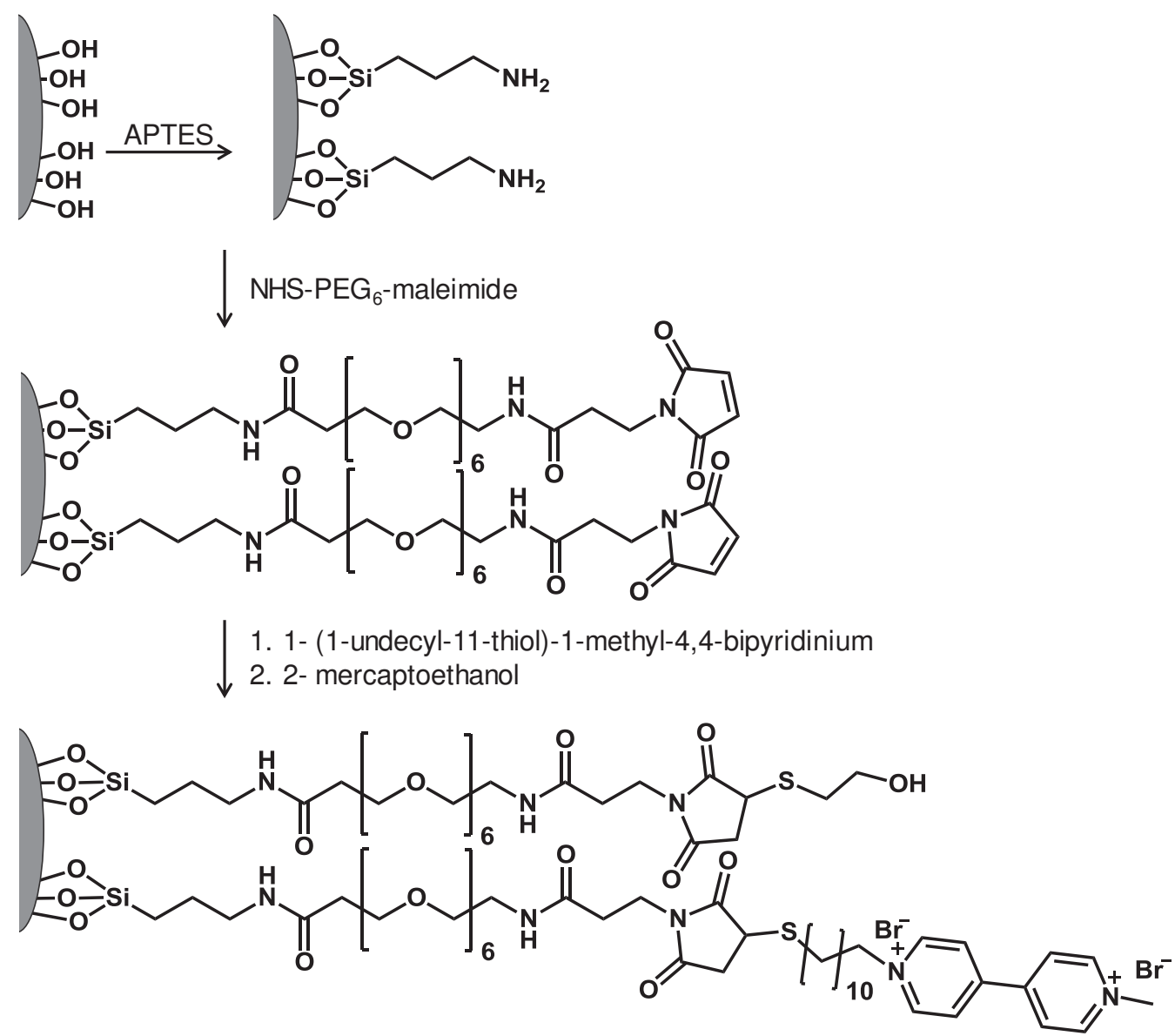

Scheme 8.2: Synthesis of methyl viologen-conjugated silica NPS $\left(\mathrm{SiO}_{2}-\mathrm{MV}\right)$.

Dynamic light scattering (DLS) and scanning electron microscopy (SEM) showed an average NP size of $61 \mathrm{~nm}$ and $52 \mathrm{~nm}$, respectively (Figure 8.1a and b). The density of guest moieties attached onto the particle surface, important for stoichiometry control during supramolecular assembly, was determined by UV/Vis spectroscopy to be $5.3 \mu \mathrm{mol}$ MV per $\mathrm{g}$ of NPs ( 900 MV/NP, approx. $1 \mathrm{MV}$ per $12 \mathrm{~nm}^{2}$ ) (Figure 8.1c). This coverage is 3.6 times higher than reported for the transferrin-functionalized silica nanoparticles, which might be attributed to the bulkier transferrin protein compared to MV. This density ensures that the guest moieties have sufficient space for supramolecular host-guest complexation with $\mathrm{CB}[8]$ and $\mathrm{Np}$.
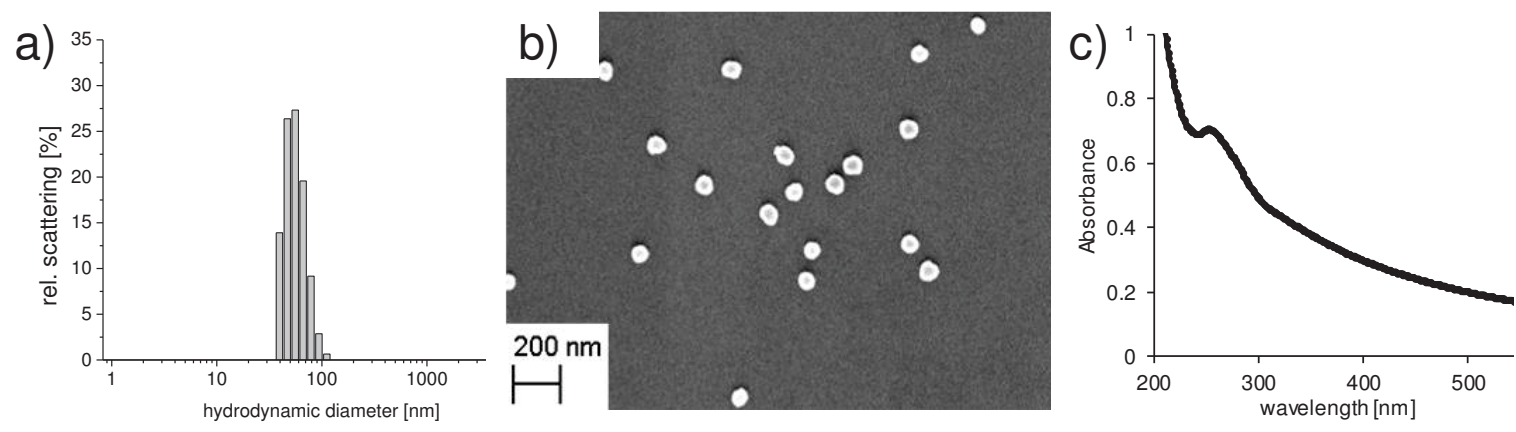

Figure 8.1: Characterization of $\mathrm{SiO}_{2}-\mathrm{MV}$ by a) DLS analysis. b) SEM analysis. c) UV/Vis spectroscopy. 


\subsubsection{Formation of SNPCs in bulk}

The formation of SNPCs in solution was studied using a $2 \mu \mathrm{M}$ concentration of CB[8], MV and $\mathrm{Np}$, while keeping the molecular recognition moieties in an equimolar 1:1:1 ratio. The relative concentrations of the monovalent $\mathrm{Np}$-PEG and the multivalent $\mathrm{Np}_{8}$-PAMAM were varied during self-assembly, while keeping the overall concentration of $\mathrm{Np}$ moieties constant. As schematically shown in Figure 8.2, SNPC formation was tested in bulk by mixing an aqueous $\mathrm{SiO}_{2}-\mathrm{MV}$ dispersion with a previously prepared aqueous solution of $\mathrm{CB}[8]$, Np-PEG $\left(\mathrm{M}_{\mathrm{w}}=5000 \mathrm{~g} / \mathrm{mol}\right)$ and $\mathrm{Np}_{8}$-PAMAM, using $25 \%, 37.5 \%$ or $50 \% \mathrm{~Np}$ from $\mathrm{Np}_{8}$-PAMAM.

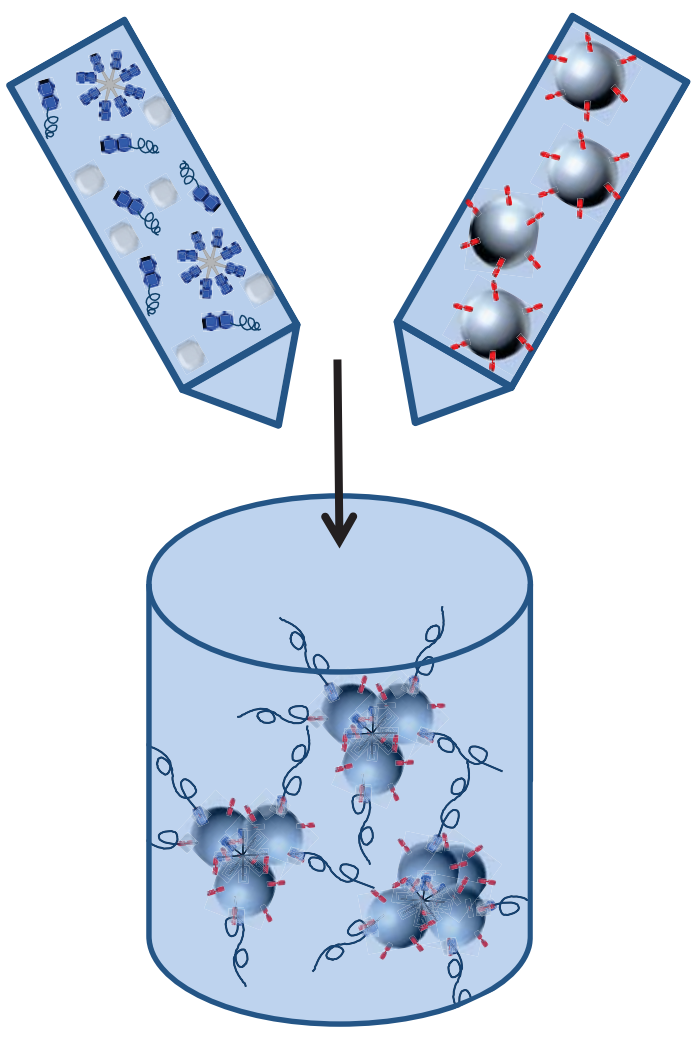

Figure 8.2: Schematic illustration of the formation of supramolecular nanoparticle clusters in bulk.

Instantaneous SNPC formation was observed $10 \mathrm{~min}$ after mixing as observed by a drastic increase of the average hydrodynamic cluster size by DLS (Figure 8.3). At the same time, the size distributions of the SNPCs were very broad (ranging from $143 \mathrm{~nm}$ to $441 \mathrm{~nm}$ and from $776 \mathrm{~nm}$ to $1813 \mathrm{~nm}$ for the $25 \%$ and $50 \% \mathrm{~Np}_{8}$-PAMAM samples, respectively). As size focusing of soft SNPs formed by the same heteroternary supramolecular complex has been observed over the course of days (Chapter 3), the size of the SNPCs was monitored in time. In contrast to soft SNPs, the batch-formed SNPCs showed complete cluster sedimentation after $14 \mathrm{~h}$. Apparently, the turbulence and fluid motion from stirring have led to uncontrolled and irreproducible NP aggregation. ${ }^{36} \mathrm{We}$ assume that, in contrast to the fully molecular SNPS, the SNPCs targeted here cannot 
equilibrate to a thermodynamic minimum because of the higher valency and the slower diffusion of the $\mathrm{SiO}_{2}-\mathrm{MV}$ NPs compared to the MV-polymer used in the soft SNPs. Therefore, kinetic control is deemed mandatory to achieve stable SNPCs.

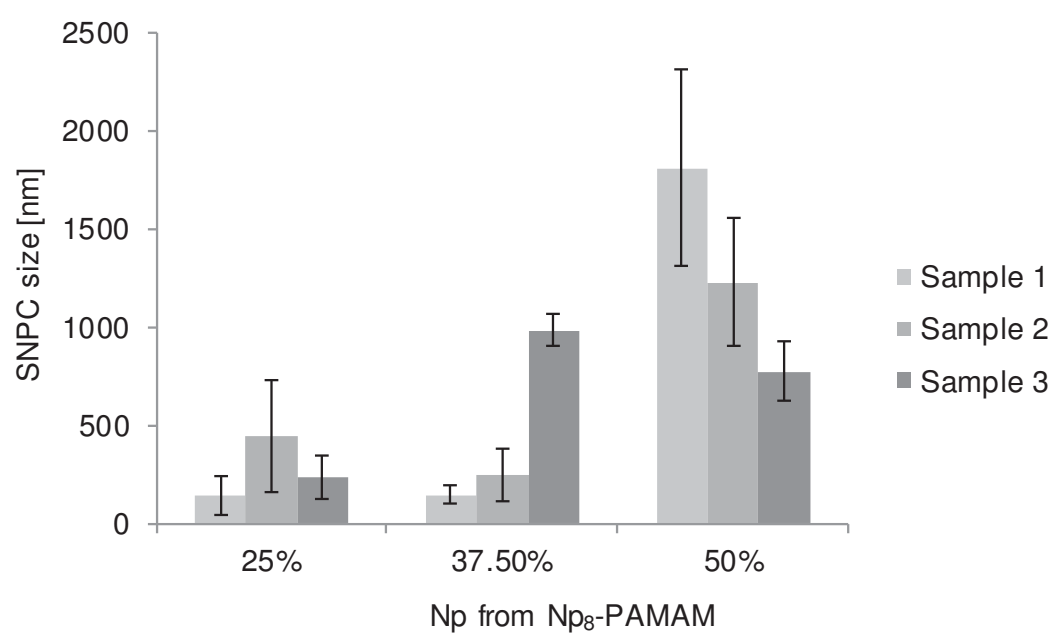

Figure 8.3: Averaged DLS sizes of SNPCS prepared in batch at a constant ratio MV:CB[8]:Np of 1:1:1, as a function of $N p$ derived from $N p_{8}$-PAMAM dendrimers used during SNPC self-assembly.

\subsubsection{Formation of SNPCs in a microreactor}

Subsequently, network aggregation of $\mathrm{SiO}_{2}-\mathrm{MV}$ was evaluated in the confined environment of a microreactor. To this end, the dispersion of $\mathrm{SiO}_{2}-\mathrm{MV}$ was injected in one of the two inlets of the microreactor, while a premixed solution of $\mathrm{CB}[8], \mathrm{Np}-\mathrm{PEG}$, and $\mathrm{Np}_{8}$-PAMAM was injected in the second inlet, and the two streams were put in laminar flow with an equal flow rate (Figure 8.4). The diffusion coefficients and the time required for the different SNPC components to diffuse over the distance of the microreactor were evaluated by the Stokes-Einstein $\left(D=\frac{k T}{6 \pi \eta R}\right)$ and the Einstein-Smoluchowski equations $\left(t=\frac{d^{2}}{2 D}\right)$, respectively (Table 8.1 ), assuming absence of complexation. 


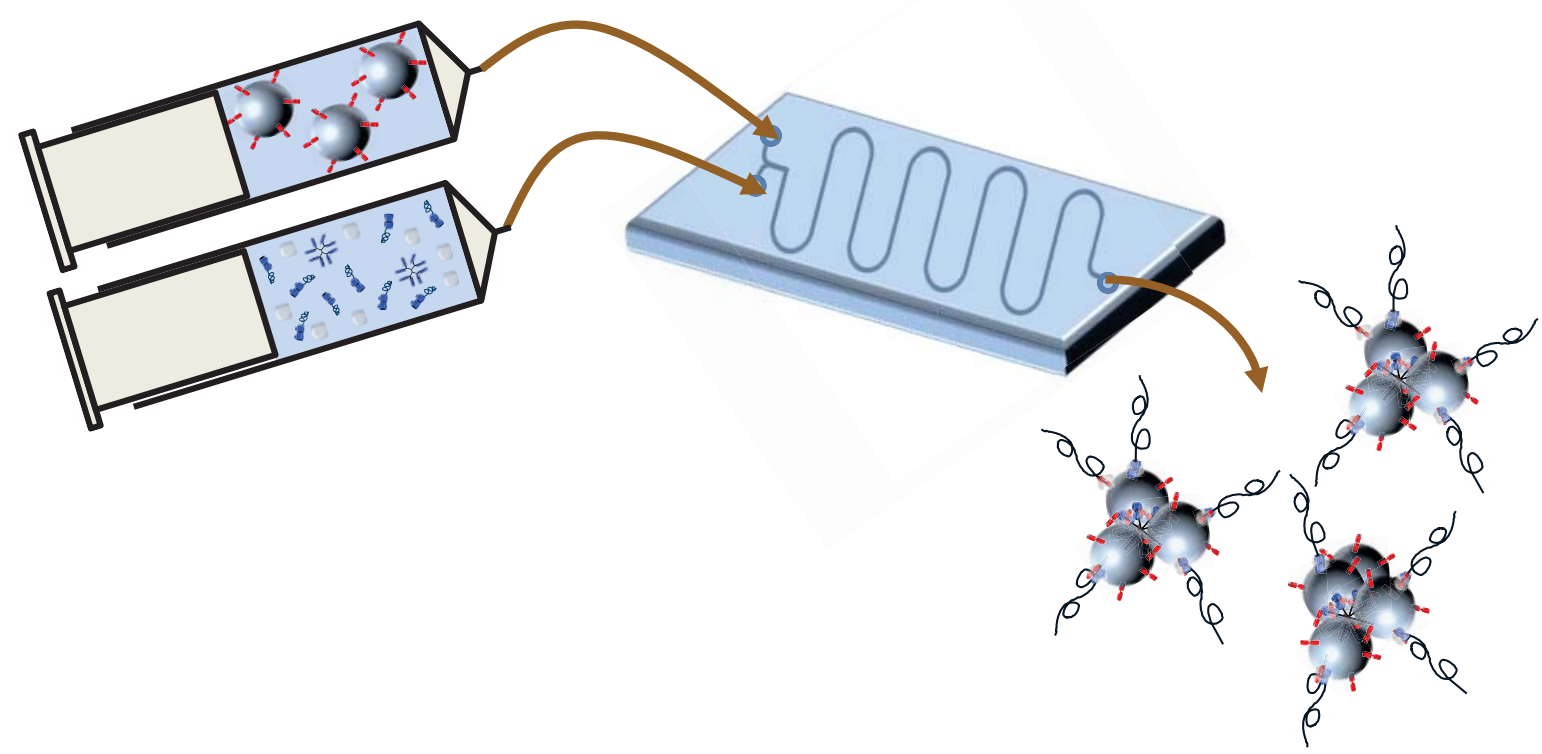

Figure 8.4: Schematic illustration of the microfluidic assisted supramolecular network aggregation of silica particles, dendrimers and PEG stopper molecules, mediated by the ternary interaction of MV and $\mathrm{Np}$ in the presence of $C B[8]$.

Table 8.1: Diffusion coefficients and diffusion times of the supramolecular building blocks used in SNPC formation in a microfluidic device.

\begin{tabular}{|c|c|c|c|}
\hline Supramolecular building block & $R(\mathrm{~m})$ & $D\left(\mathrm{~m}^{2} / \mathrm{s}\right)^{\mathrm{a}}$ & $t(\mathrm{~s})^{\mathrm{b}}$ \\
\hline $\mathrm{SiO}_{2}-\mathrm{MV}$ & $2.5 \times 10^{-8}$ & $8.6 \times 10^{-12}$ & 911 \\
\hline $\mathrm{CB}[8]^{37}$ & $8.8 \times 10^{-10}$ & $2.5 \times 10^{-10}$ & 32 \\
\hline PAMAM G1 & $9.3 \times 10^{-10}$ & $2.3 \times 10^{-10}$ & 34 \\
\hline Np-PEG $(1000 \mathrm{~g} / \mathrm{mol})^{39}$ & $9.7 \times 10^{-10}$ & $2.2 \times 10^{-10}$ & 35 \\
\hline Np-PEG $(5000 \mathrm{~g} / \mathrm{mol})^{40}$ & $2.3 \times 10^{-9}$ & $9.4 \times 10^{-11}$ & 83 \\
\hline
\end{tabular}

a $\left.D=\frac{k T}{6 \pi \eta R}\right)$, where, $k$ is the Boltzmann constant $\left(1.38 \times 10^{-23} \mathrm{~m}^{2} \mathrm{~kg} \mathrm{~s}^{-2} \mathrm{~K}^{-1}\right), T$ is the temperature $(293.15 \mathrm{~K}), \eta$ is the viscosity of water $\left(1.002 \times 10^{-3} \mathrm{~kg} \mathrm{~s}^{-1} \mathrm{~m}^{-1}\right), R$ is the radius of the building blocks. ${ }^{\mathrm{b}} t=\frac{d^{2}}{2 D^{2}}$, where $d$ indicates half of the microchannel width.

Given the dimensions of the device and the flow rates used here, the diffusion rate of $\mathrm{SiO}_{2}-\mathrm{MV}$ is too small to expect appreciable penetration of the particles into the other stream. In contrast, the diffusion of $\mathrm{Np}_{8}$-PAMAM, Np-PEG ( $\left.\mathrm{M}_{\mathrm{w}} 1000 \mathrm{~g} / \mathrm{mol}\right)$, and CB[8] are comparable, allowing these molecules to diffuse into the complete NP flow within approximately $35 \mathrm{~s}$ (Figure 8.5). The diffusion rate of $\mathrm{Np}-\mathrm{PEG}\left(\mathrm{M}_{\mathrm{w}} 5000 \mathrm{~g} / \mathrm{mol}\right)$ is calculated to be somewhat lower. The assembly formation in a microreactor is controlled by the diffusion of the molecular components into the nanoparticle stream, as exemplified by the diffusion profiles assumed in the absence of complexation. Based on these results, we decided to start clustering experiments with a residence time of $60 \mathrm{~s}$. 


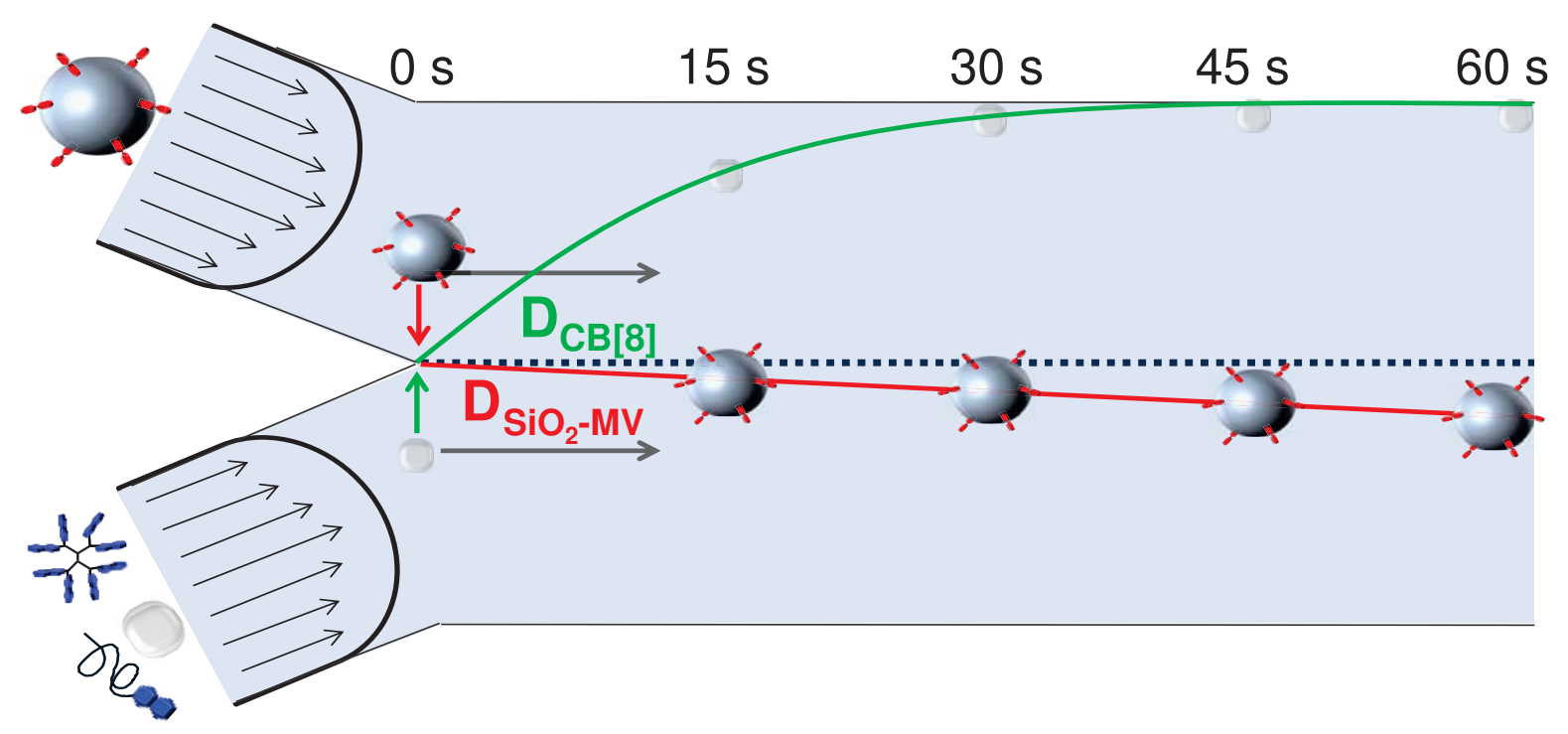

Figure 8.5: Schematic illustration of the particle $\left(\mathrm{SiO}_{2}-\mathrm{MV}\right.$, red) and molecular (here shown for $\mathrm{CB}[8]$ in green, similar for $\mathrm{NP}-\mathrm{PEG}$ and $\mathrm{Np}_{8}-\mathrm{PAMAM}$ ) diffusion profiles in time over the width of the microfluidic reactor (top view).

\subsubsection{Size tuning of SNPCs by varying the stoichiometry}

In contrast to the experiments performed in bulk conditions, distinct SNPCs were observed at a residence time of $60 \mathrm{~s}$. DLS and SEM show clearly that the resulting cluster size can be tuned over a large dynamic range by varying the ratio of the two $\mathrm{Np}$ components while keeping the overall stoichiometry of $\mathrm{CB}[8] / \mathrm{MV} / \mathrm{Np}$ constant (Figure 8.6). By increasing the amount of $\mathrm{Np}$ from $\mathrm{Np}_{8}$-PAMAM from $25 \%$ to $62.5 \%$, while decreasing the amount of Np from Np-PEG $\left(M_{w} 5000 \mathrm{~g} / \mathrm{mol}\right)$ from $75 \%$ to $37.5 \%$, an increase in SNPC size from $125 \pm 10 \mathrm{~nm}$ to $370 \pm 100 \mathrm{~nm}$ is observed by DLS, while SEM indicates a similar tuning range from $65 \pm 20 \mathrm{~nm}$ to $256 \pm 146 \mathrm{~nm}$. 


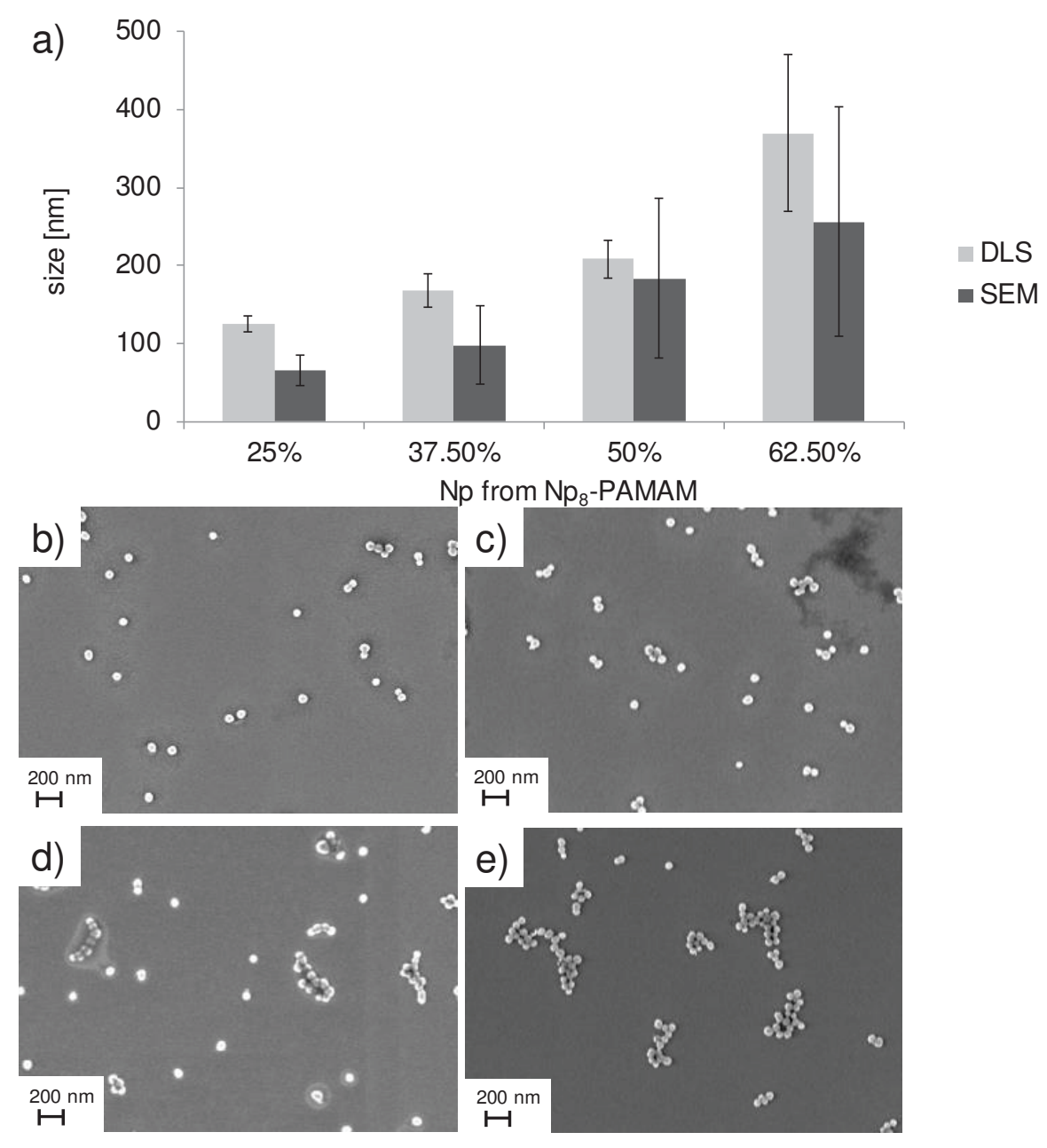

Figure 8.6: Size determination of SNPCS induced by ternary host-guest interactions ( $2 \mu M$ of interacting moieties, 60 s residence time). a) Average NP cluster diameter by DLS (light grey bars) and SEM (dark grey bars). SEM images (b-e) of the resulting SNPCS, as a function of the Np content derived from $\mathrm{Np}_{8}-\mathrm{PAMAM}$ (b: $25 \%, \mathrm{c:} 37.5 \%$, d: $50 \%$, e: $62.5 \%$ ) used during microfluidic assisted NP network aggregation.

Furthermore, the ratio of the monovalent and multivalent binding partners does not significantly influence the uniformity of the formed aggregates: DLS shows polydispersity indices (PDI) of the observed SNPCs between 0.30 and 0.42 irrespective of the sample (Table 8.2).

Table 8.2: PDI observed by DLS of the SNPCS prepared with different concentrations of the Npbearing guest components.

\begin{tabular}{|c|c|}
\hline Np from $\mathrm{Np}_{8}$-PAMAM & PDI \\
\hline $25.0 \%$ & $0.30-0.40$ \\
\hline $37.5 \%$ & $0.35-0.42$ \\
\hline $50.0 \%$ & $0.30-0.42$ \\
\hline $62.5 \%$ & $0.30-0.40$ \\
\hline
\end{tabular}


Generally, the clustering solution was collected from the outlet of the microreactor for 40 min to obtain sufficient sample for DLS studies. In contrast, all SEM samples were prepared by collecting the as-assembled SNPC solution directly from the microreactor onto the substrate. To assess the validity of the DLS results, SEM control experiments were carried out on samples collected for $40 \mathrm{~min}$. These showed that the observed cluster sizes do not vary between the instantaneous measurements and the measurements prepared from collecting over $40 \mathrm{~min}$ (Figure 8.7). The good correlation between the DLS and SEM data, as well as this control, confirm therefore the strong kinetic trapping already mentioned above. In contrast, all SNPCs showed sedimentation from the suspension overnight. This is not unexpected as even the initial, nonaggregated $\mathrm{SiO}_{2}-\mathrm{MV}$ NPs start to sediment within a few hours.
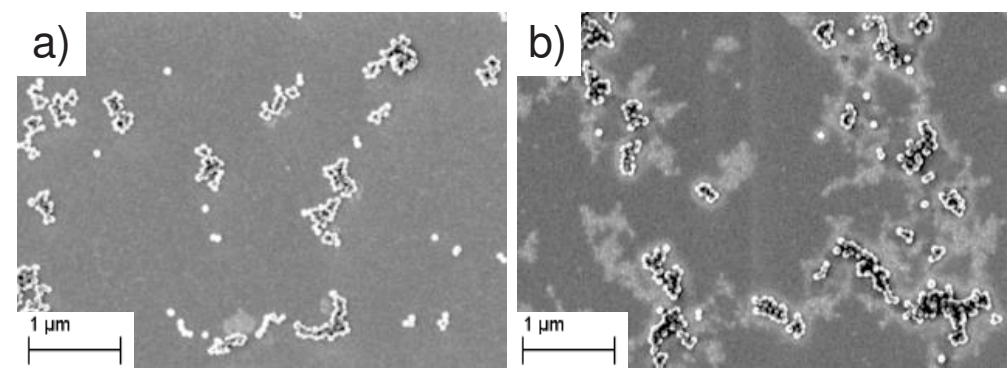

Figure 8.7: SEM images of SNPC solution with $62.5 \%$ of the Np content derived from N $p_{8}$-PAMAM: a) obtained directly from the microfluidic reactor and b) obtained 40 min after starting the supramolecular self-assembly. Residence time: $60 \mathrm{~s}$.

To evaluate the effect of the Np-bearing components on microfluidic assisted selfassembly of SNPCs, SNPC formation was carried out using either $100 \% \mathrm{~Np}$ from Np-PEG or $100 \% \mathrm{~Np}$ from $\mathrm{Np}_{8}$-PAMAM. In contrast to the previously shown data, individual nonaggregated particles were observed by carrying out SNPC formation in the absence of $\mathrm{Np}_{8}$-PAMAM (Figure $8.8 \mathrm{a}$ and $\mathrm{b}$ ), whereas large aggregates were observed by SEM (Figure 8.8c) upon mixing $\mathrm{SiO}_{2}-\mathrm{MV}, \mathrm{CB}[8]$ and $\mathrm{Np}_{8}$-PAMAM in the absence of Np-PEG. These results show that $\mathrm{Np}_{8}$-PAMAM is required to crosslink the $\mathrm{SiO}_{2}-\mathrm{MV}$, whereas uncontrolled aggregation is prohibited by competition with the monovalently interactiing Np-PEG.

a)

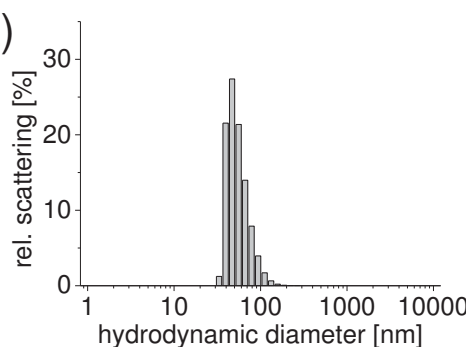

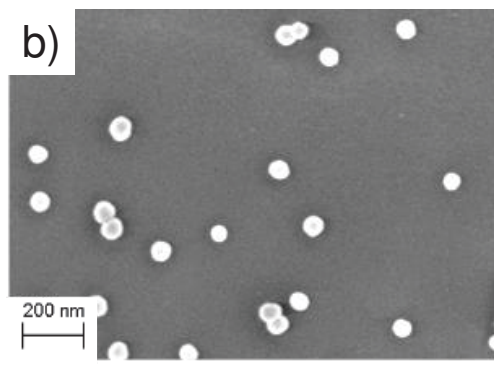

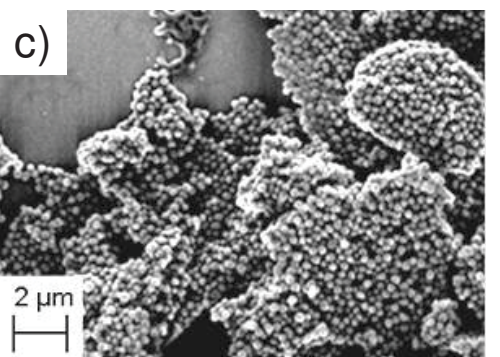

Figure 8.8: DLS and SEM images of self-assembly experiments: a) DLS and b) SEM of $\mathrm{SiO}_{2}-M V$, $C B[8]$, and Np-PEG $\left(M_{w}=5000 \mathrm{~g} / \mathrm{mol}\right)$ in the absence of $\mathrm{Np}_{8}-$ PAMAM. c) SEM of $\mathrm{SiO}_{2}-\mathrm{MV}, \mathrm{CB}[8]$, and $\mathrm{Np}_{8}$-PAMAM in the absence of $\mathrm{Np}$-PEG. Residence time: $60 \mathrm{~s}$. 
In another control experiment, $\mathrm{SiO}_{2}-\mathrm{MV}$ and both $\mathrm{Np}$-bearing components $(62.5 \% \mathrm{~Np}$ from $\mathrm{Np}_{8}$-PAMAM) were used, but with $\mathrm{CB}[7]$ instead of $\mathrm{CB}[8]$. The smaller $\mathrm{CB}[7]$ is too small to include both the MV and Np guest moieties in its cavity, and consequently the heteroternary complex cannot be formed. Indeed, no SNPC formation was observed by DLS or SEM (Figure 8.9a and b). A similar control was performed using bare silica nanoparticles instead of the MV-functionalized ones. Also here (Figure 8.9c and d), only non-aggregated NPs were observed.

a)

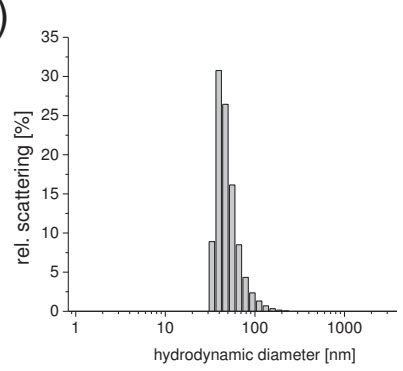

c)

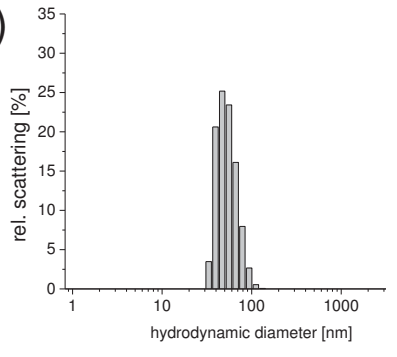

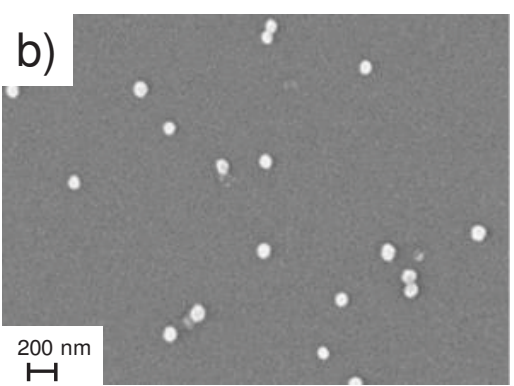

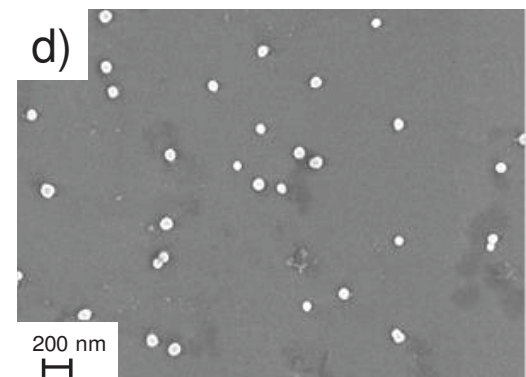

Figure 8.9: DLS and SEM images of clustering experiments: a) and b) with $\mathrm{SiO}_{2}-\mathrm{MV}, \mathrm{CB}$ [7] (instead of $\mathrm{CB}[8]), 62.5 \% \mathrm{~Np}$ from $\mathrm{Np}_{8}-\mathrm{PAMAM}$, and $37.5 \% \mathrm{~Np}$ from $\mathrm{Np}-\mathrm{PEG}$; c) and d) with $\mathrm{SiO}_{2}-\mathrm{OH}, \mathrm{CB}[8]$, $62.5 \% \mathrm{~Np}$ from $\mathrm{Np}_{8}-\mathrm{PAMAM}$, and $37.5 \% \mathrm{~Np}$ from $\mathrm{Np}$-PEG. Residence time: $60 \mathrm{~s}$.

In summary, SNPCs can be formed within a microfluidic reactor in which diffusive mixing leads to controllable aggregation. Their formation is based on specific ternary complex formation, and the size of the resulting clusters is strongly dependent on the ratio between the mono- and multivalent Np guest species. The dependence of the resulting SNPC size on the fraction of $\mathrm{Np}_{8}$-PAMAM in the $\mathrm{Np}$-bearing building blocks can be ascribed to the competition between monovalent and multivalent host-guest interactions in the presence of $\mathrm{CB}[8]$ and $\mathrm{SiO}_{2}-\mathrm{MV}$. The amount of inter- and intramolecular binding events increases with the increased content of multivalent dendrimers, which leads to larger SNPC structures. The monovalent Np-PEG is essential to terminate the network formation and the accompanying uncontrolled aggregation as it blocks interaction sites and shields the crosslinked cores by the sterics of the PEG chains.

\subsubsection{Size tuning of the SNPCs by varying the residence time}

To investigate the effect of residence time and thus the diffusion time of the components on the size and kinetics of the assembly formation, clustering experiments were carried 
out with $\mathrm{SiO}_{2}-\mathrm{MV}, \mathrm{CB}[8], \mathrm{Np}_{8}-\mathrm{PAMAM}$, and Np-PEG $\left(\mathrm{M}_{\mathrm{w}}=5000 \mathrm{~g} / \mathrm{mol}\right)$ at a constant $62.5 \% \mathrm{~Np}$ derived from $\mathrm{Np}_{8}$-PAMAM. As seen in Figure 8.10, the cluster sizes observed by DLS varied strongly and in a non-trivial manner: a maximum in cluster size was observed at a $30 \mathrm{~s}$ residence time. The non-linear trend is tentatively explained by the calculated diffusion times of the different molecular building blocks. The diffusion time of Np-PEG $\left(\mathrm{M}_{\mathrm{w}}=5000 \mathrm{~g} / \mathrm{mol}\right)$ is about 2.5 times slower than the diffusion time of $\mathrm{Np}_{8}$-PAMAM and $\mathrm{CB}$ [8] (Table 8.1), which may result in less controlled cluster formation at the shorter residence times due to faster diffusion of the crosslinking $\mathrm{Np}_{8}$-PAMAM compared to the cluster-stabilizing Np-PEG. For example, by using a residence time of $30 \mathrm{~s}, \mathrm{CB}[8]$ and the dendrimer can diffuse over almost the complete width of the NP stream, whereas the monovalent Np-PEG $\left(M_{w}=5000 \mathrm{~g} / \mathrm{mol}\right)$ can only penetrate approximately $25 \%$ into the other fluid stream. In contrast, at $60 \mathrm{~s}$ residence time, all components are able to penetrate the full NP stream, thereby explaining the observed size control at this residence time.

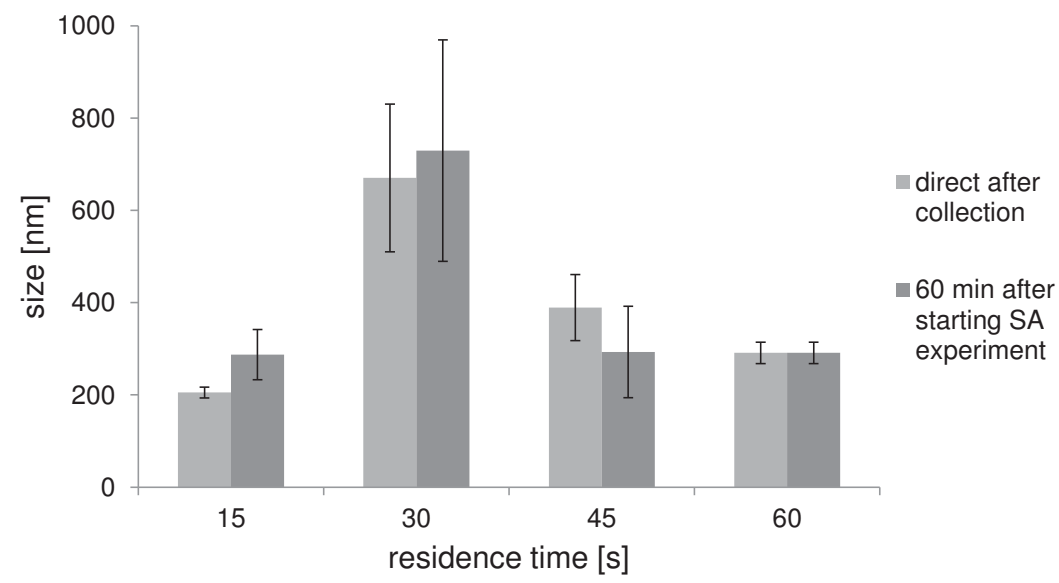

Figure 8.10: SNPC size by DLS upon assembly of $\mathrm{SiO}_{2}-M V, C B[8], N p_{8}-P A M A M$, and $N p-P E G\left(M_{w}=\right.$ $5000 \mathrm{~g} / \mathrm{mol}$ ) at $2 \mu \mathrm{M}$ 1:1:1 CB[8]/MV/Np (62.5\% $\mathrm{Np}$ from $\left.\mathrm{Np}_{8}-\mathrm{PAMAM}\right)$ as a function of residence time directly after SNPC assembly (light grey) or 60 min after starting SNPC formation (dark grey).

In order to eliminate this diffusion effect, we used Np-PEG with a $M_{w}$ of 1000 instead of the earlier used 5000. The shorter Np-PEG polymer has a diffusion time that is very similar to $\mathrm{Np}_{8}$-PAMAM and $\mathrm{CB}[8]$ (Table 8.1). Upon cluster formation using Np-PEG (1000 $\mathrm{g} / \mathrm{mol}$ ), $\mathrm{Np}_{8}-\mathrm{PAMAM}, \mathrm{CB}[8]$ and $\mathrm{SiO}_{2}-\mathrm{MV}$, a monotonous dependence of SNPC size with residence time is observed (Figure 8.11a and $c-f$ ), by both DLS and SEM. This confirms that the equal diffusion times of the molecular components govern the simultaneous assembly and termination of the clusters. Furthermore, DLS shows that the PDI strongly depends on the mixing time and decreases upon increase of the overall residence time of the components within the microreactor (Table 8.3). Nevertheless, the DLS size measurements only provide averages of cluster sizes, whereas the SEM images provide more detail on the size distribution. Many images show the presence of non-aggregated particles in the SNPC samples, which prompted us to evaluate their fraction as a function 
of residence time (Figure 8.11b). At short residence times, the diffusion of the molecular components is insufficient to pass the whole width of the microfluidic channel. Consequently, not all $\mathrm{SiO}_{2}-\mathrm{MV}$ NPs are integrated in SNPCs. This is confirmed by the results shown in Figure $8.11 \mathrm{~b}$, whereby the fraction of non-aggregated NPs decreases strongly from 30 to $5 \%$ upon increasing the residence time from 15 to $60 \mathrm{~s}$. By excluding the non-assembled NPs from the formed SNPCs, correction of the size determinations by SEM is possible. As shown in Figure 8.11a, a clear SNPC size increase and a decrease in the SNPC size distribution is observed by excluding the unbound $\mathrm{SiO}_{2}-\mathrm{MV}$. These effects are more pronounced for the samples prepared with lower residence times, since the fractions of unbound NPs are higher in this case.
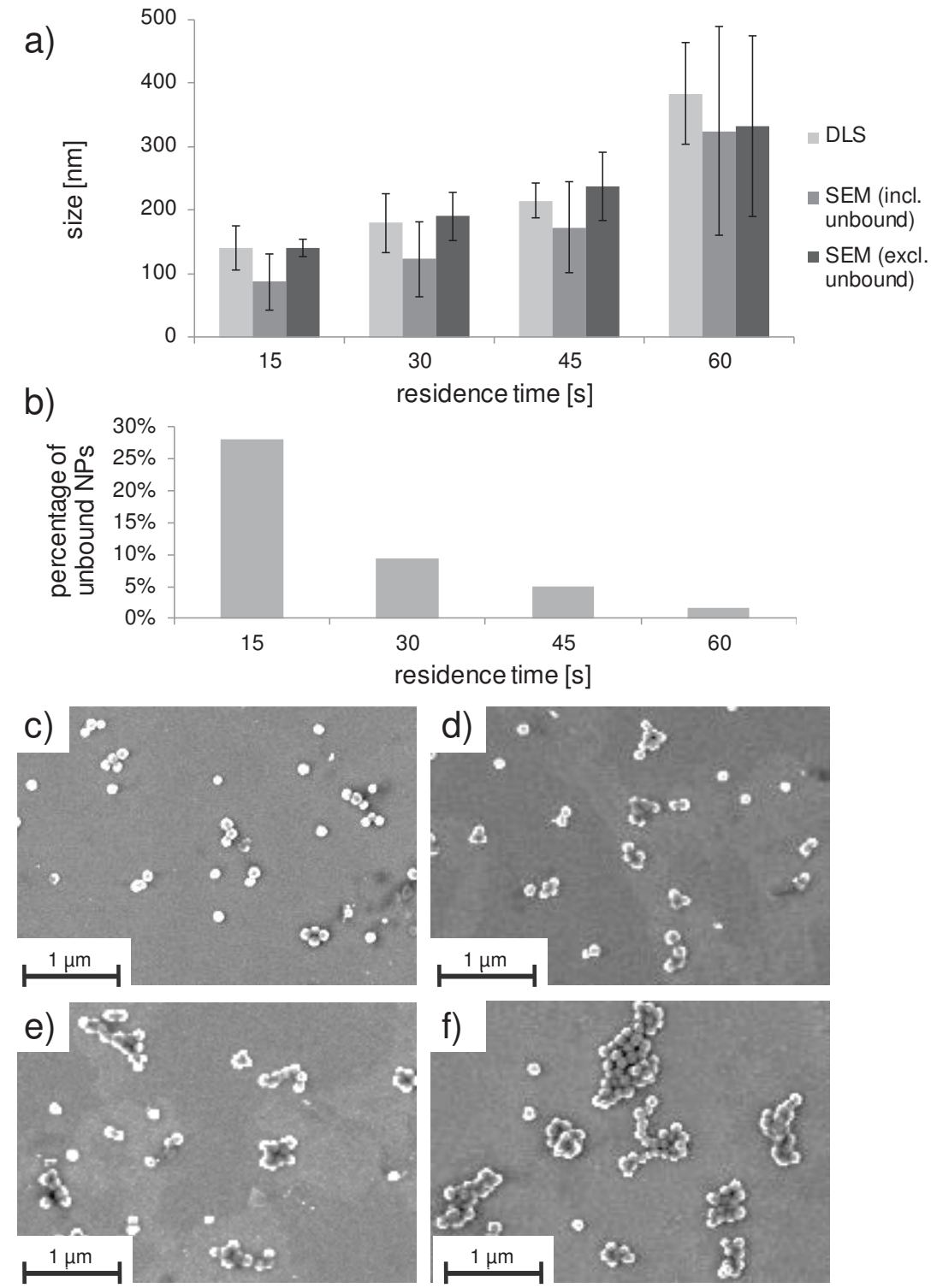

Figure 8.11: a) SNPC size and b) fraction of unbound $\mathrm{SiO}_{2}-\mathrm{MV}$ NPS, by assembly of $\mathrm{SiO}_{2}-\mathrm{MV}, \mathrm{CB}[8]$,

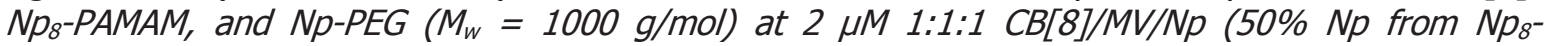
$P A M A M)$ as a function of residence time, as measured by DLS (light grey) and SEM (grey bars including all observed $\mathrm{SiO}_{2}-\mathrm{MV}$ NPS and dark grey bars excluding unbound $\mathrm{SiO}_{2}-\mathrm{MV} N \mathrm{NS}$ ). SEM images (c-f) of the resulting SNPCS prepared within a microfluidic device at different residence times of the two interacting streams (c: $15 \mathrm{~s}, \mathrm{~d}: 30 \mathrm{~s}, \mathrm{e}: 45 \mathrm{~s}, \mathrm{f}: 60 \mathrm{~s}$ ). 
Table 8.3: PDI observed by DLS of the SNPCS prepared at different residence times.

\begin{tabular}{|c|c|}
\hline Residence time (s) & PDI \\
\hline 15 & $0.58-0.72$ \\
\hline 30 & $0.51-0.72$ \\
\hline 45 & $0.23-0.36$ \\
\hline 60 & $0.28-0.35$ \\
\hline
\end{tabular}

\subsubsection{Size tuning of the SNPCs by varying the concentrations of the building blocks}

Because SNPC formation is dependent on the diffusion of the molecular components into the NP stream, we decided to investigate the effect of the concentration of these components on the assembly process. Therefore, the stoichiometry of CB[8] and Np with respect to MV was varied under constant flow conditions (residence time $30 \mathrm{~s}$ ), while keeping the content of $\mathrm{Np}$ from $\mathrm{Np}_{8}$-PAMAM constant at $50 \%$. By increasing the $\mathrm{CB}[8] / \mathrm{MV} / \mathrm{Np}$ ratio from $1: 1: 1$ to $3: 1: 3$, the overall observed size of the SNPCs increased from $180 \pm 46 \mathrm{~nm}$ to $354 \pm 60 \mathrm{~nm}$ and from $123 \pm 58 \mathrm{~nm}$ to $205 \pm 70 \mathrm{~nm}$ by DLS (Figure 8.12a) and SEM (Figure 8.12b-d), respectively. Further increase of the $\mathrm{CB}[8] / \mathrm{MV} / \mathrm{Np}$ ratio to $5: 1: 5$ led to large SNPCs and visible precipitation (Figure 8.12e). The PDI of the samples increased upon increasing ratio of $\mathrm{CB}[8]$ and $\mathrm{Np}$ with respect to MV (Table 8.4). Irrespective of the composition, the samples showed the presence of $10 \%$ of unbound $\mathrm{SiO}_{2}-\mathrm{MV}$ NPs. The latter result confirms that the extent of nonaggregated NPs is solely dependent on the diffusion rates of the molecular components and thus on the penetration depth of the molecular components into the NP stream (Figure 8.13a). On the other hand, this result and the observed increase of SNPC size upon increasing concentrations of the molecular components (Figure 8.13b) indicate that the molecular components are not fully incorporated into the SNPCs, since complete use at 1:1:1 stoichiometry should have been accompanied by an independence of aggregate size upon further increase of the concentration and by a depletion of the molecular components and thus a lower penetration depth (and higher fraction of unbound $\mathrm{SiO}_{2}-$ MV NPs) at lower concentrations. 

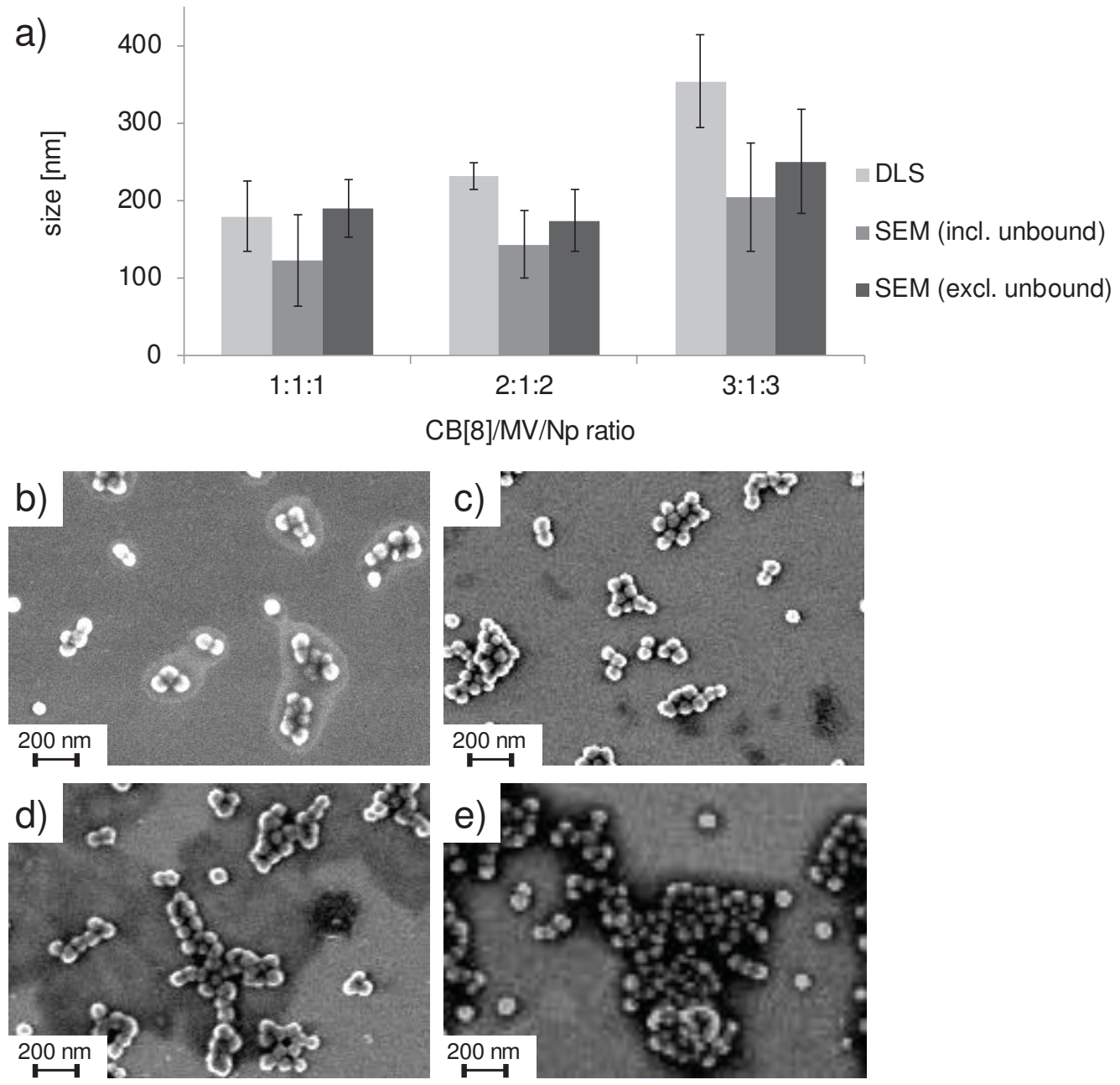

Figure 8.12: a) SNPC size by assembly of $\mathrm{SiO}_{2}-\mathrm{MV}, \mathrm{CB}[8], \mathrm{Np} \mathrm{p}_{8}$-PAMAM, and Np-PEG (M $\mathrm{M}_{w}=1000$ $\mathrm{g} / \mathrm{mol}$ ) at different $\mathrm{CB}[8] / \mathrm{MV} / \mathrm{Np}$ ratios (50\% $\mathrm{Np}$ from $\mathrm{Np}_{8}$-PAMAM) as measured by DLS (light grey bars) and SEM (grey bars including all observed $\mathrm{SiO}_{2}-M V$ NPS and dark grey bars excluding unbound $\mathrm{SiO}_{2}-M V$ NPS). SEM images (b-e) of the resulting SNPCS prepared within a microfluidic device at different $C B[8] / M V / N p$ ratios (b: 1:1:1; c: 2:1:2; d: 3:1:3; e: 5:1:5). Residence time: 60 s.

Table 8.4: PDI observed by DLS of the SNPCs prepared with different host-guest stoichiometries.

\begin{tabular}{|c|c|}
\hline $\mathrm{CB}[8] / \mathrm{MV} / \mathrm{Np}$ & $\mathrm{PDI}$ \\
\hline $1: 1: 1$ & $0.53-0.68$ \\
\hline $2: 1: 2$ & $0.54-0.89$ \\
\hline $3: 1: 3$ & $0.80-0.97$ \\
\hline $5: 1: 5$ & $1.25-1.54$ \\
\hline
\end{tabular}


a)
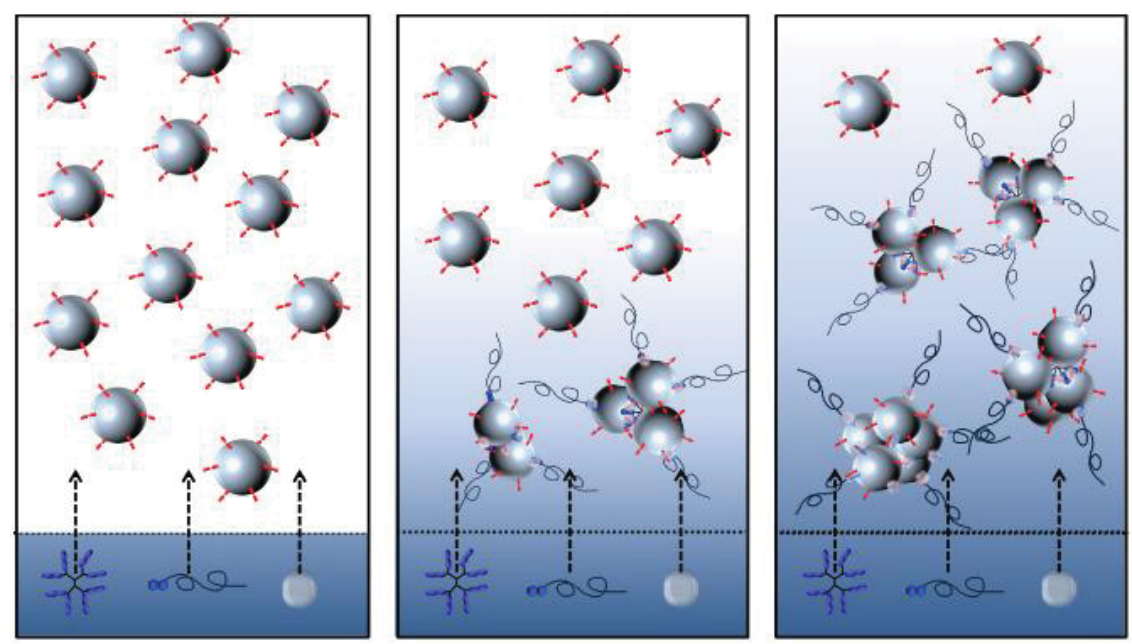

b)

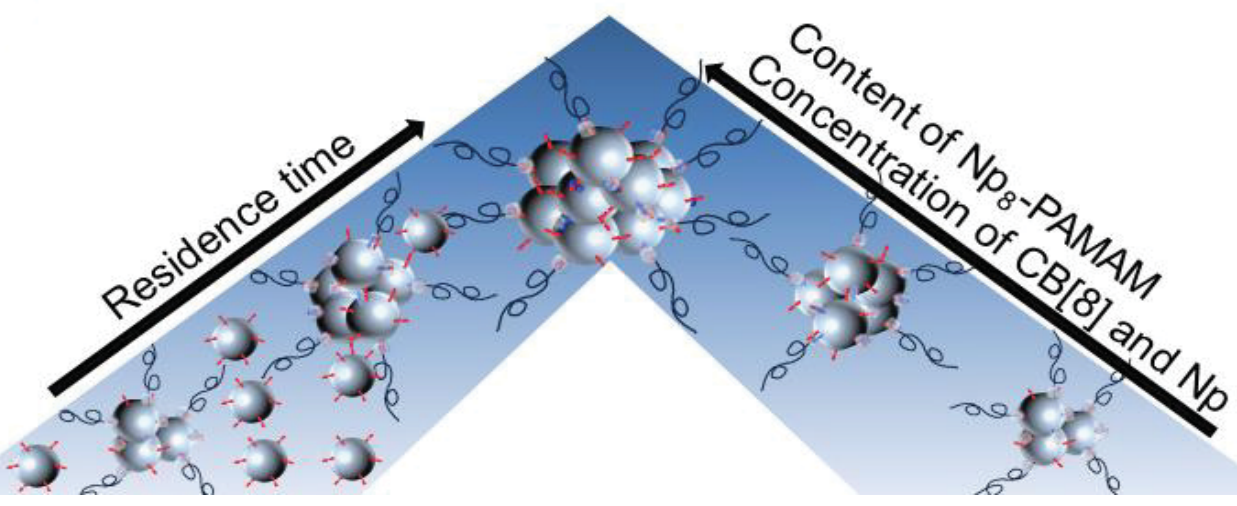

Figure 8.13: a) Schematic illustration of SNPC formation inside the microfluidic reactor depending on the diffusion of $\mathrm{Np}_{8}-\mathrm{PAMAM}, \mathrm{Np}-\mathrm{PEG}$, and $\mathrm{CB}[8]$ within the $\mathrm{SiO}_{2}-\mathrm{MV}$ stream. (b) Dependence of SNPC size and of the fraction of unbound $\mathrm{SiO}_{2}-\mathrm{MV}$ on the residence time, the relative fraction of multivalent $\mathrm{Np}_{8}-\mathrm{PAMAM}$, and the concentrations of the molecular components.

\subsection{Conclusions}

In conclusion, the formation of SNPCs driven by ternary host-guest interactions of CB[8], $\mathrm{MV}$, and $\mathrm{Np}$ has been demonstrated within a microfluidic device. As schematically shown in Figure $8.13 a$, the diffusive mixing is strongly determined by the diffusion profile of the molecular components $\mathrm{CB}[8], \mathrm{Np}_{8}$-PAMAM, and Np-PEG into the $\mathrm{SiO}_{2}-\mathrm{MV} \mathrm{NP}$ stream. The size of the resulting SNPCs is strongly depending on the content of multivalently interacting Np dendrimers, the residence time of the interacting building blocks within the microchannel, and the stoichiometry of the ternary host-guest binding partners (Figure 8.13b). The residence time variation as well as the discrepancy between clustering experiments carried out in batch and in the microfluidic reactor show that the supramolecular host-guest assembly, is to a large extent, kinetically controlled. Interestingly, the molecular weight of the PEG stopper provides an additional kinetic control parameter to the assembly process because a longer length leads to slower diffusion into the NP stream and a concomitantly later termination of the clusters. 
Overall, the combination of microfluidic technology with the design and control parameters provided by supramolecular chemistry provides an advanced platform for the tunable assembly of NP network aggregates, which may prove beneficial for the preparation of functional nanostructures for materials engineering.

\subsection{Acknowledgements}

Rajesh Munirathinam is gratefully acknowledged for the collaboration on the assembly experiments in the microfluidic devices. Jan Eijkel is gratefully acknowledged for fruitful discussions. Mark Smithers is acknowledged for the SEM images.

\subsection{Experimental Section}

\subsubsection{Materials}

Starting materials for particle synthesis were obtained from Sigma-Aldrich and used as received. The bifunctional linker $\mathrm{NHS}-(\mathrm{PEG})_{6}$-maleimide was purchased from Fisher Scientific. 1-(1-Undecyl-11-thiol)-1-methyl-4,4-bipyridinium was prepared according to a literature procedure. ${ }^{35}$ Cucurbit[8]uril (CB[8]) was purchased from Strem Chemicals and its purity was assessed by microcalorimetric titration against paraquat. In all experiments, the solutions required for SNPC formation were prepared in distilled water purified by MilliQ Advantage A10, Millipore, $R=18.2 \mathrm{M} \Omega \mathrm{cm}^{-1}$, before usage. Single-side polished silicon(100) wafers required for the preparation of scanning electron microscopy (SEM) samples were purchased from OKMETIC.

\subsubsection{Synthetic procedures}

Preparation of methyl viologen-functionalized silica nanoparticles (SiO2-MV)

In accordance to a literature procedure, ${ }^{40}$ bare silicon nanoparticles were prepared by adding $3.8 \mathrm{~mL}$ tetraethyl orthosilicate to a mixture of $5.7 \mathrm{~mL} \mathrm{NH} \mathrm{NH}_{4} \mathrm{OH} 114 \mathrm{~mL}$ ethanol under stirring. This resulted in nanoparticles of approximately $60 \mathrm{~nm}$. Under argon, 2.3 $\mathrm{mL}$ (3-aminopropyl)triethoxysilane (APTES) was added under vigorous stirring to $120 \mathrm{~mL}$ of the previously prepared silica nanoparticles in ethanol. The dispersion was stirred overnight and the excess of APTES removed by repeated NP centrifugation and redispersion in $60 \mathrm{~mL}$ ethanol. The particles were dried under reduced pressure and stored at $4{ }^{\circ} \mathrm{C}$ as white solid. The amino-functionalized $\mathrm{SiO}_{2} \mathrm{NPs}$ were dispersed in degassed HEPES $20 \mathrm{mM}$ buffer ( $\mathrm{pH}$ 7.4) to prepare a dispersion with a concentration of $10 \mathrm{mg} / \mathrm{mL}$. $10 \mathrm{~mL}$ of the particle solution was added to an equal volume of $23 \mathrm{mM}$ of NHS-PEG 6 -maleimide in 20 mM HEPES buffer ( $\mathrm{pH}$ 7.4). The dispersion was sonicated for $5 \mathrm{~min}$ and further shaken overnight. The opaque dispersion was purified by centrifugation and subsequent redispersion of the NPs, twice in HEPES buffer $(\mathrm{pH} 7.4)$ and twice in HEPES buffer ( $\mathrm{pH} \mathrm{6.8)}$ to remove the bivalent linker. Subsequently, the $\mathrm{SiO}_{2}$-maleimide 
NPs were dispersed in degassed HEPES buffer ( $\mathrm{pH}$ 6.8) to obtain a concentration of 5 $\mathrm{mg} / \mathrm{mL}$. 1-(1-Undecyl-11-thiol)-1-methyl-4,4-bipyridinium dibromide (1.7 mg, $3.1 \mu \mathrm{mol}$ ) was dissolved in $3 \mathrm{~mL}$ degassed HEPES buffer ( $\mathrm{pH} \mathrm{6.8)}$ and added to $15 \mathrm{~mL}$ of the $\mathrm{SiO}_{2}-$ maleimide dispersion while stirring. After shaking the dispersion for $2 \mathrm{~h}$, the unfunctionalized terminal maleimide groups were capped by reaction with $10 \mu \mathrm{L}$ of 2mercaptoethanol for $5 \mathrm{~min}$. The resulting $\mathrm{SiO}_{2}-\mathrm{MV} \mathrm{NPs}$ were purified by repeated centrifugation, once in HEPES buffer ( $\mathrm{pH}$ 6.8), twice in ethanol and twice in water. Finally, the nanoparticles were freeze-dried and kept as a white solid in the fridge.

\subsubsection{Self-assembly procedures}

Supramolecular cluster formation in batch reaction

For the preparation of size-tunable supramolecular nanoparticle clusters (SNPCs), the $\mathrm{SiO}_{2}-\mathrm{MV}$ nanoparticles were dispersed in MilliQ water to obtain a concentration of $0.8 \mathrm{mg}$ $\mathrm{NP} / \mathrm{mL}$. The solution was sonicated until only individual NPs were observed by DLS. In addition, $8 \mu \mathrm{M}$ solution of $\mathrm{CB}[8]$ and various ratios of aqueous solutions of Np-PEG (5000 $\mathrm{g} / \mathrm{mol}$ ) and $\mathrm{Np}_{8}$-PAMAM in DMSO were prepared, maintaining the $\mathrm{MV}: \mathrm{CB}[8]: \mathrm{Np}$ ratio at $1: 1: 1$. For example, to form the SNPCs containing $50 \% \mathrm{~Np}$ derived from dendrimer, 500 $\mu \mathrm{L} \mathrm{SiO}_{2}-\mathrm{MV}$ was added to a previously prepared solution of $250 \mu \mathrm{L} \mathrm{Np}-\mathrm{PEG}(4 \mu \mathrm{M}), 10 \mu \mathrm{L}$ $\mathrm{Np}_{8}$-PAMAM $(12.5 \mu \mathrm{M})$ and $250 \mu \mathrm{L} \mathrm{CB}[8](8 \mu \mathrm{M})$. The sample was mixed using a vortex mixer and analyzed by DLS at $10 \mathrm{~min}$ and $14 \mathrm{~h}$ after mixing.

\section{Supramolecular cluster formation in a microreactor}

A dispersion of $\mathrm{SiO}_{2}-\mathrm{MV}$ in water and a solution containing $\mathrm{CB}[8]$, Np-PEG $\left(\mathrm{M}_{\mathrm{w}}=5000\right.$ or $1000 \mathrm{~g} \mathrm{~mol}^{-1}$ ), and $\mathrm{Np}_{8}$-PAMAM in water were prepared as mentioned above. The concentrations of the different components were set such that all three binding motifs $\mathrm{CB}[8], \mathrm{MV}$, and $\mathrm{Np}$ were present in equimolar amounts and at a $2 \mu \mathrm{M}$ concentration after mixing. For different residence times, the flow rates were adjusted accordingly.

Formation of SNPCs by varying the ratio of Np-functionalized components derived from $\mathrm{Np}_{8}$-PAMAM and Np-PEG

Different concentrations of Nps derived from Np$p_{8}$-PAMAM (25, 37.5, 50 and 62.5\%) and Np-PEG $\left(M_{w}=5000 \mathrm{~g} / \mathrm{mol} ; 75,62.5,50\right.$ and 37.5\%), respectively, were prepared before mixing, maintaining the $\mathrm{MV}: \mathrm{CB}[8]: \mathrm{Np}$ ratio at $1: 1: 1$. The dispersion of $\mathrm{SiO}_{2}-\mathrm{MV}$ was injected in one of the two inlets of a microchannel, while the premixed solution of $\mathrm{CB}[8]$ and Np-bearing components was injected in the second inlet. The two fluid streams were flowed in parallel with an equal flow rate of $4 \mu \mathrm{L} / \mathrm{min}$, which corresponds to a residence time of $60 \mathrm{~s}$. The SNPC solution was collected until a volume of $350 \mu \mathrm{L}$ required for DLS analysis was obtained. SEM samples were prepared by collecting the SNPC solution directly from the outlet of the microreactor. 


\section{Formation of SNPCs by varying the residence time}

The experiments carried out with variable residence times of the mixing solutions inside the microreactor, were carried out using a steady-state origin of the Np-bearing components (either $50 \%$ or $62.5 \% \mathrm{~Np}$ from $\mathrm{Np}_{8}$-PAMAM). Therefore, the $\mathrm{SiO}_{2}-\mathrm{MV}$ dispersion and the mixture of $\mathrm{CB}[8]$, Np-PEG $(5000 \mathrm{~g} / \mathrm{mol}$ and $1000 \mathrm{~g} / \mathrm{mol})$ and $\mathrm{Np}_{8}$ PAMAM were prepared and mixed within the microfluidic reactor. The flow rates of two inlets were equal and were varied from $16.0,8.0,5.2$ to $4.0 \mu \mathrm{L} / \mathrm{min}$, which resulted in residence times of $15,30,45$, and 60 s, respectively.

\section{Formation of SNPCs by varying the stoichiometry of the building blocks}

A constant content of $\mathrm{MV}$ and $50 \%$ of $\mathrm{Np}$ derived from $\mathrm{Np}_{8}$-PAMAM dendrimer were used in all the experiments, while increasing the amount of $\mathrm{CB}[8]$ and Np stoichiometrically. For example, to obtain an overall $\mathrm{CB}[8] / \mathrm{MV} / \mathrm{Np}$ ratio of $2: 1: 2$, a $0.8 \mathrm{mg} / \mathrm{mL}$ dispersion of $\mathrm{SiO}_{2}-\mathrm{MV}$ in water was used in one inlet, and a premixed solution of $\mathrm{CB}[8](8 \mu \mathrm{M}), \mathrm{Np}-$ PEG $\left(\mathrm{M}_{\mathrm{w}}=1000 \mathrm{~g} / \mathrm{mol}, 4 \mu \mathrm{M}\right)$, and Np $\mathrm{N}_{8}$-PAMAM $(0.5 \mu \mathrm{M})$ in water was used in the other inlet. The other ratios (3:1:3 and 5:1:5) were made similarly, using accordingly higher concentrations of $\mathrm{CB}[8]$ and the Np components. All experiments were carried out with a residence time of $60 \mathrm{~s}$.

\section{SNPC formation in the absence of multivalent $\mathrm{Np}_{8}$-PAMAM}

A dispersion of $\mathrm{SiO}_{2}-\mathrm{MV}(0.8 \mathrm{mg} / \mathrm{mL})$ in water was injected into one of the inlets and a mixture of $\mathrm{CB}[8](4 \mu \mathrm{M})$ and Np-PEG $(4 \mu \mathrm{M})$ in water was injected into the second inlet using an overall residence time of $60 \mathrm{~s}$.

\section{SNPC formation in the absence of Np-PEG}

A dispersion of $\mathrm{SiO}_{2}-\mathrm{MV}(0.8 \mathrm{mg} / \mathrm{mL})$ in water was injected into one of the inlets and a mixture of $\mathrm{CB}[8](4 \mu \mathrm{M})$ and $\mathrm{Np}_{8}$-PAMAM $(0.5 \mu \mathrm{M})$ in water was injected into the other inlet. The clustering was carried out using an overall residence time of 60 s.

\section{SNPC formation in the presence of $\mathrm{CB}[7]$ instead of $\mathrm{CB}[8]$}

A dispersion of $\mathrm{SiO}_{2}-\mathrm{MV}(0.8 \mathrm{mg} / \mathrm{mL})$ in water was injected into one of the inlets and a mixture of $\mathrm{CB}$ [7] $(4 \mu \mathrm{M})$, Np-PEG $(1.5 \mu \mathrm{M})$, and $\mathrm{Np}_{8}$-PAMAM $(0.313 \mu \mathrm{M})$ in water was injected into the second inlet using an overall residence time of $60 \mathrm{~s}$.

\section{SNPC formation in presence of $\mathrm{SiO}_{2}-\mathrm{OH}$ instead of $\mathrm{SiO}_{2}-\mathrm{MV}$}

A dispersion of $\mathrm{SiO}_{2}-\mathrm{OH}(0.8 \mathrm{mg} / \mathrm{mL})$ in water was injected into one of the inlets and a pre-mixed solution of Np-PEG $(1.5 \mu \mathrm{M}), \mathrm{CB}[8](4 \mu \mathrm{M})$ and $\mathrm{Np}_{8}$-PAMAM $(0.313 \mu \mathrm{M})$ in water was injected into the second inlet with an overall residence time of $60 \mathrm{~s}$ 


\subsubsection{Equipment}

\section{DLS analysis (DLS)}

DLS experiments were performed with a Nanotrac wave by Anaspec operating with a Microtrac FLEX Operating Software at $25^{\circ} \mathrm{C}$ using a laser wavelength of $780 \mathrm{~nm}$ at a scattering angle of $90^{\circ}$. The observed sizes and standard deviations of the SNPCs were based on the average number distributions of minimum five individual measurements per sample. Three samples were measured for each reported NP formulation. In total, particles and SNPCs with sizes ranging from 0.8 to $6400 \mathrm{~nm}$ were detected.

\section{Scanning electron microscopy (SEM)}

All SEM images were taken with a Carl Zeiss Merlin scanning electron microscope. Samples were prepared by drop-casting an approximately 10 times diluted SNPC dispersion onto a cleaned silicon wafer. This was done by adding $2 \mu \mathrm{L}$ of water followed by collecting a small droplet directly from the outlet of the microreactor. The dispersion was completely removed $10 \mathrm{~s}$ after addition and analyzed without further treatment. The sizes of at least 150 individual clusters were used to determine the average size of the SNPCs. Additionally, another average SNPC size was determined by excluding all unbound NPs.

\section{$\underline{\text { UV/Vis spectroscopy }}$}

UV/Vis absorption spectra were recorded using a Perkin Elmer Lambda 850 UV-Vis spectrometer. The content of $\mathrm{MV}$ on the $\mathrm{SiO}_{2}-\mathrm{MV}$ NPs was determined using the characteristic UV adsorption band of MV at $259 \mathrm{~nm}$. Therefore a dispersion of $\mathrm{SiO}_{2}-\mathrm{MV}$ in water $(0.5 \mathrm{mg} / \mathrm{mL})$ was prepared and analyzed by UV spectroscopy (Figure $8.1 \mathrm{c})$. The content of MV was determined using a calibration curve of 1-(1-undecyl-11-thiol)-1methyl-4,4-bipyridinium dibromide in water, after subtracting the enhanced background signal induced by NP scattering. ${ }^{41}$

\section{Microreactor set-up}

In all microreactor experiments, the sample solutions were mobilized by means of a PHD 22/2000 series syringe pump (Harvard Apparatus, United Kingdom) equipped with $250 \mu \mathrm{L}$ flat tip syringes (Hamilton). Syringes were connected to fused silica capillaries (100 $\mu \mathrm{m}$ i.d., $362 \mu \mathrm{m}$ o.d., Polymicro Technologies) by means of Upchurch Nanoport ${ }^{\mathrm{TM}}$ assembly parts (i.e., Nano-Tight ${ }^{\mathrm{TM}}$ unions and fittings, Upchurch Scientific Inc. USA). During the experiments the microreactor was placed in a home-built chip holder designed for fitting fused silica fibers into the inlet/outlet chip reservoirs by means of commercially available Upchurch NanoportTM assembly parts. A glass microreactor with dimensions of $645.6 \mathrm{~mm}$ length, $52 \mu \mathrm{m}$ depth, $254 \mu \mathrm{m}$ channel top width, and $150 \mu \mathrm{m}$ channel bottom width, and a residual volume of $13 \mu \mathrm{L}$ were purchased from Micronit Microfluidics (Enschede, The Netherlands). 


\subsection{References}

1. B. A. Grzybowski, C. E. Wilmer, J. Kim, K. P. Browne and K. J. M. Bishop, Soft Matter, 2009, 5, 1110-1128.

2. M. Grzelczak, J. Vermant, E. M. Furst and L. M. Liz-Marzán, ACS Nano, 2010, 4, 35913605.

3. K. J. M. Bishop, C. E. Wilmer, S. Soh and B. A. Grzybowski, Small, 2009, 5, 1600-1630.

4. M. D. Yilmaz and J. Huskens, Soft Matter, 2012, 8, 11768-11780.

5. E. Masson, X. Ling, R. Joseph, L. Kyeremeh-Mensah and X. Lu, RSC Advances, 2012, 2, 1213-1247.

6. F. Cheng, Z. Tao, J. Liang and J. Chen, Chemistry of Materials, 2007, 20, 667-681.

7. D. Patra, F. Ozdemir, O. R. Miranda, B. Samanta, A. Sanyal and V. M. Rotello, Langmuir, 2009, 25, 13852-13854.

8. A. B. Descalzo, R. Martínez-Máñez, F. Sancenón, K. Hoffmann and K. Rurack, Angewandte Chemie International Edition, 2006, 45, 5924-5948.

9. H. Li, D.-X. Chen, Y.-L. Sun, Y. B. Zheng, L.-L. Tan, P. S. Weiss and Y.-W. Yang, Journal of the American Chemical Society, 2012, 135, 1570-1576.

10. X. Chen, S. G. Parker, G. Zou, W. Su and Q. Zhang, ACS Nano, 2010, 4, 6387-6394.

11. M.-C. Daniel and D. Astruc, Chemical Reviews, 2003, 104, 293-346.

12. R. Mejia-Ariza and J. Huskens, Journal of Materials Chemistry B, 2014, 2, 210-216.

13. G. M. Whitesides, Nature, 2006, 442, 368-373.

14. K. S. Elvira, X. C. i Solvas, R. C. R. Wootton and A. J. deMello, Nature Chemistry, 2013, 5, 905-915.

15. P. Yager, T. Edwards, E. Fu, K. Helton, K. Nelson, M. R. Tam and B. H.Weigl, Nature, 2006, 442, 412-418.

16. B. Zheng, J. D. Tice and R. F. Ismagilov, Analytical Chemistry, 2004, 76, 4977-4982.

17. Y. Song, J. Hormes and C. S. S. R. Kumar, Small, 2008, 4, 698-711.

18. M. Numata and T. Kozawa, Chemistry - A European Journal, 2013, 19, 12629-12634.

19. F. S. Majedi, M. M. Hasani-Sadrabadi, S. Hojjati Emami, M. A. Shokrgozar, J. J. VanDersarl, E. Dashtimoghadam, A. Bertsch and P. Renaud, Lab on a Chip, 2013, 13, 204-207.

20. M. Lu, Y.-P. Ho, C. L. Grigsby, A. A. Nawaz, K. W. Leong and T. J. Huang, ACS Nano, 2013, 8, 332-339.

21. J. He, L. Wang, Z. Wei, Y. Yang, C. Wang, X. Han and Z. Nie, ACS Applied Materials \& Interfaces, 2013, 5, 9746-9751.

22. Z. Yu, C.-F. Wang, L. Ling, L. Chen and S. Chen, Angewandte Chemie International Edition, 2012, 51, 2375-2378.

23. C.-W. Wang, D. Sinton and M. G. Moffitt, Journal of the American Chemical Society, 2011, 133, 18853-18864.

24. G. Schabas, H. Yusuf, M. G. Moffitt and D. Sinton, Langmuir, 2008, 24, 637-643.

25. R. Karnik, F. Gu, P. Basto, C. Cannizzaro, L. Dean, W. Kyei-Manu, R. Langer and O. C. Farokhzad, Nano Letters, 2008, 8, 2906-2912.

26. G. Tresset, C. Marculescu, A. Salonen, M. Ni and C. Iliescu, Analytical Chemistry, 2013, $85,5850-5856$. 
27. N. Hassan, V. Cabuil and A. Abou-Hassan, Angewandte Chemie International Edition, 2013, 52, 1994-1997.

28. M. Numata and T. Kozawa, Chemistry- $A$ European Journal, 2014, 20,6234-6240.

29. K. Liu, H. Wang, K.-J. Chen, F. Guo, W.-Y. Lin, Y.-C. Chen, D. L. Phung, H.-R. Tseng and C. K.-F. Shen, Nanotechnology, 2010, 21, 445603.

30. H. Wang, K. Liu, K.-J. Chen, Y. Lu, S. Wang, W.-Y. Lin, F. Guo, K.-i. Kamei, Y.-C. Chen, M. Ohashi, M. Wang, M. A. Garcia, X.-Z. Zhao, C. K. F. Shen and H.-R. Tseng, ACS Nano, 2010, 4, 6235-6243.

31. A. Kulkarni, R. VerHeul, K. DeFrees, C. J. Collins, R. A. Schuldt, A. Vlahu and D. H. Thompson, Biomaterials Science, 2013, 1, 1029-1033.

32. J. Zhang, R. J. Coulston, S. T. Jones, J. Geng, O. A. Scherman and C. Abell, Science, 2012, 335, 690-694.

33. A. Salvati, A. S. Pitek, M. P. Monopoli, K. Prapainop, F. B. Bombelli, D. R. Hristov, P. M. Kelly, C. Aberg, E. Mahon and K. A. Dawson, Nature Nanotechnology, 2013, 8, 137-143.

34. V. Mahalingam, S. Onclin, M. Péter, B. J. Ravoo, J. Huskens and D. N. Reinhoudt, Langmuir, 2004, 20, 11756-11762.

35. H. Kotani, K. Ohkubo, Y. Takai and S. Fukuzumi, The Journal of Physical Chemistry B, 2006, 110, 24047-24053.

36. M. Elimelech, J. Gregory, X. Jia, R. A. Williams, J. Gregory, X. Jia and R. A. Williams, in Particle Deposition \& Aggregation, eds. M. Elimelech, J. Gregory, X. Jia, R. A. Williams, J. Gregory, X. Jia and R. A. Williams, Butterworth-Heinemann, Woburn, 1995, pp. 157-202.

37. J. Kim, I.-S. Jung, S.-Y. Kim, E. Lee, J.-K. Kang, S. Sakamoto, K. Yamaguchi and K. Kim, Journal of the American Chemical Society, 2000, 122, 540-541.

38. V. Jiménez, J. Gavín and J. Alderete, Structural Chemistry, 2012, 23, 123-128.

39. M. P. J. Dohmen, A. M. Pereira, J. M. K. Timmer, N. E. Benes and J. T. F. Keurentjes, Journal of Chemical \& Engineering Data, 2007, 53, 63-65.

40. J. K. Armstrong, R. B. Wenby, H. J. Meiselman and T. C. Fisher, Biophysical Journal, 87, 4259-4270.

41. S. J. Leach and H. A. Scherag, Journal of the American Chemical Society, 1960, 47904792. 


\section{Summary}

Supramolecular nanoparticles (SNPS) have gained significant interest in nanoscience because stable, but reversible three-dimensional assemblies can be used for a variety of applications, ranging from stimuli-responsive and self-healing materials to biomedical applications. In particular, soft SNPs are promising candidates in biomedicine for drug delivery and diagnosis. The versatility of the SNP toolbox concept enables the easy decoration of SNPs with specific celltargeting molecules and the incorporation of several small molecules, such as drugs or imaging agents, into the SNPs. Based on this strategy, drug and gene delivery vehicles have been developed, some of which are currently being tested in clinical studies as cancer therapeutics. At the same time, many of the underlying self-assembly and colloidal properties of these particles remain unclear, as well as the integration of stimulus-responsive properties for controlled disassembly and release. The work described in this thesis aims at the development of novel strategies for the formation of soft SNPs, while specific attention is paid to the understanding of SNP formation, stabilization, size tunability and triggered disassembly.

In the first part of this thesis (Chapters 3 and 4), a novel fabrication method for soft SNPs has been reported. Stabilized by multiple host-guest interactions between cucurbit[8]uril, methyl viologen and naphthol, thermodynamically controlled and time and temperature dependent SNP assembly was observed. These SNPs showed size tuning by the interplay between multivalent core and monovalent or divalent (Chapter 4) shell-forming stopper molecules. The length of the hydrophilic shell-forming polymer does not have a significant effect on the SNP size, as long the polymer is long enough to provide colloidal stability. Using a divalent stopper instead of a monovalent one slowed down the assembly kinetics significantly.

In the second part of this thesis (Chapters 5-7) stimuli-responsive SNPs have been developed based on the photoswitchable, heteroternary complex of cucurbit[8]uril, methyl viologen and azobenzene. Photoisomerization of azobenzene led to rupture of the supramolecular complex and thereby to UV-triggered SNP disintegration. In combination with the naphthol building blocks reported in Chapter 3, the SNP assembly strategy allowed SNP assembly in a toolbox approach, whereby the photoswitchable Azo was placed selectively in the core, the shell or both. Furthermore, by using a non-fouling azobenzene-conjugated zwitterion as the stopper, long PEG 
stoppers could be avoided and colloidal stability was observed at high salt and high protein concentrations. A remarkable $\mathrm{pH}$-dependent and reversible SNP aggregation was observed.

Finally, Chapter 8 describes the kinetically controlled self-assembly of supramolecular nanoparticle clusters (SNPCs) mediated by heteroternary host-guest interaction of methyl viologen-functionalized silica nanoparticles, cucurbit[8]uril and naphthol building blocks by diffusive mixing in a microreactor. The resulting SNPC size was shown to depend strongly on the residence time, the competition between multivalent and monovalent naphthol entities, and the stoichiometry of the host and guest binding partners.

In summary, the work described in this thesis provides a general strategy for the development of supramolecular nanoparticles mediated by cucurbit[8]uril host-guest interactions. Based on the heteroternary inclusion of guest molecules into cucurbit[8]uril, the formation of SNPs with highly controllable sizes, diverse shell functionalities and different responses to stimuli have been reported. The time and temperature dependent SNP self-assembly, as well as the different disassembly pathways give new insight towards the formation and disintegration of SNPs and their dynamics. This is essential for the development of effective carriers that can potentially be used in biomedical applications and meet the stringent and multifaceted demands of living systems. We expect that, upon inclusion of active compounds, these SNPs can provide a new generation of functional, biomedically relevant nanodevices. 


\section{Samenvatting}

Supramoleculaire nanodeeltjes (SND's) zijn van groot belang in de nanowetenschap omdat deze als stabiele en reversibele driedimensionale structuren gebruikt kunnen worden voor verschillende toepassingen zoals in schakelbare en zelf-helende materialen en in biomedische toepassingen. Met name zachte SND's zijn veelbelovende kandidaten voor medicijnafgifte en diagnostiek. De veelzijdigheid van het concept van SND's maakt de functionalisatie mogelijk van SND's met celherkennende moleculen of het insluiten van verschillende kleine moleculen, zoals medicijnen of contrastreagentia. Met behulp van deze strategie zijn verschillende systemen ontwikkeld voor medicinale en gentherapie, waarvan sommige op dit moment klinisch getest worden voor toepassing in het behandelen van kanker. Tegelijkertijd zijn vele van de onderliggende zelf-assemblerende en colloïdale eigenschappen van SND's onopgehelderd, evenals het implementeren van schakelbare eigenschappen voor het gestuurd afbreken van SND's en het daarmee vrijlaten van hun lading. Het werk beschreven in dit proefschrift richt zich op de ontwikkeling van nieuwe strategieën voor de vorming van zachte SND's, terwijl vooral aandacht is geschonken aan het begrip van de zelf-assemblage van de SND's, hun stabilisatie, het sturen van hun grootte en de gestuurde desintegratie.

In het eerste deel van dit proefschrift (hoofdstukken 3 en 4) is een nieuwe methode voor de vorming van zachte SND's beschreven. De vorming van SND's, die gestabiliseerd worden door meervoudige gastheer-gast-interacties tussen cucurbit[8]uril, methylviologeen en naftol, bleek thermodynamisch gecontroleerd te zijn en tevens afhankelijk van de temperatuur. De grootte van deze SND's kon gestuurd worden door middel van een samenspel tussen de multivalente kern en de monovalente of divalente (hoofdstuk 4) schilvormende stoppermoleculen. De lengte van het hydrofiele stopper-polymeer heeft geen significant effect op de grootte van de SND's, zolang het polymeer voldoende lengte heeft om voor colloïdale stabiliteit te zorgen. Het gebruik van de divalente in plaats van de monovalente stopper vertraagde de assemblagesnelheid aanzienlijk.

In het tweede gedeelte van dit proefschrift (hoofdstukken 5-7) zijn schakelbare SNDs ontwikkeld, gebaseerd op de lichtgevoelige, heteroternaire complexen van cucurbit[8]uril, methylviologeen en azobenzeen. Fotoisomerisatie van het azobenzeen onder UV-licht leidde tot 
de afbraak van het supramoleculaire complex en daarmee tot de desintegratie van de SND's. In combinatie met de naftolbouwstenen beschreven in hoofdstuk 3 leidde het SND-concept tot een veelzijdige SND-assemblage, waarbij het lichtgevoelige azobenzeen selectief in de kern, in de schil of in beide ingebouwd kon worden. Bovendien konden lange PEG-stoppers vermeden worden indien gebruik werd gemaakt van een anti-fouling azobenzeen-geconjugeerd zwitterion als stoppermolecuul, waarbij colloïdale stabiliteit waargenomen werd bij hoge zout- en eiwitconcentraties. Tevens is er een opmerkelijke $\mathrm{pH}$-afhankelijke en reversibele SND-aggregatie waargenomen.

Hoofdstuk 8 tenslotte beschrijft de kinetisch gecontroleerde zelf-assemblage van supramoleculaire clusters van nanodeeltjes (SCND's) gedreven door heteroternaire gastheergast-interacties tussen methylviologeen-gefunctionaliseerde silica-nanodeeltjes, cucubit[8]uril en naftol-bouwstenen bij het diffusief mengen in een microreactor. De resulterende grootte van de SCND's bleek sterk afhankelijk te zijn van de verblijftijd in de microreactor, de competitie tussen de multivalente en monovalente naftol-moleculen en de stoichiometrie van de complementaire gastheer- en gast-eenheden.

Samenvattend biedt het werk beschreven in dit proefschrift een algemene strategie voor de ontwikkeling van SND's gedreven door gastheer-gast-interacties met cucurbit[8]uril. De vorming van SND's, gebaseerd op de heteroternaire interactie van gastmoleculen met cucurbit[8]uril, heeft geleid tot met een goed controleerbare grootte, tot verschillende functionalisaties van de schil en tot verschillende manieren van schakelen van de assemblage. De tijd- en temperatuurafhankelijke assemblage van SND's en de verschillende routes voor hun desintegratie geven nieuwe inzichten in de vorming en afbraak van SND's en hun dynamiek. Dit is essentieel voor de ontwikkeling van effectieve medicijnafgiftesystemen voor mogelijke biomedische toepassingen, die moeten voldoen aan de strenge en veelzijdige eisen van levende systemen. We verwachten dat deze SND's, bij het insluiten van actieve componenten, verder ontwikkeld kunnen worden tot een nieuwe generatie van functionele en biomedisch relevante nanosystemen. 


\section{Acknowledgements}

Last but not least, it is time to acknowledge all the people who have supported me not just during my PhD studies but also during the whole time I have spent in Enschede. During this time I have met so many people, which were not just scientifically but also personally very important for me.

First and foremost, I would like to express my gratitude and appreciation to my promotor Jurriaan. Thanks for convincing me to start my PhD. After four years I am sure that this was the right decision, the perfect project and you have been the best supervisor I could have imagined for me. The open and free atmosphere gave me the opportunity to work on my own ideas and become an independent researcher. I will never forget when you were saying: "Ik ben niet getrouwd met $\mathrm{CB}$, dat was jouw keuze!" I truly appreciate how you taught me to ask the right questions (and hope I do so now) and guide me as smoothly as possible throughout all the challenges passing along the way. I really enjoyed our inspiring and mostly funny work meetings and your calmness to get me back on the track when I was again a bit overreacting. Finally I have to thank you particularly for the last year. I was not that productive as normal and struggling with my not favourite task- THE WRITING, but you kept motivating me with your full guidance and never put me under pressure. Jurriaan, hartelijk bedankt!

But many more people were involved to finish this thesis, successfully. Pascal, I did my Master project with you and you have been involved in the dual-responsive SNP project, but besides, your door was always open for any other question I had. Wim, after my Bachelor project I didn't want to work with microfluidics anymore, but in the end I could not get rid of it completely as it is visible in Chapter 8. Thanks for all the funny chats we had and the countless times you opened me the door to the office/lab. Melissa, thanks for all the nice (not only baby) chats and for carefully correcting my thesis. I hope you will enjoy your new job and get your family together in Mainz, as soon as possible. Although not directly involved in the research presented in this book, I would like to express my gratitude to the other scientific Sans staff members Tibor, Jeroen and Nathalie. 
Furthermore, in my opinion the groups would not run without our technicians. Richard, Marcel, Regine, Bianca and Tieme-thanks for all the help and support during the daily laboratory life and all your struggles to provide "clean water". Especially I have to name Richard, for all the technical and scientific support starting from my Bachelor project on. I'm sure you have been one reason why I was continuing with my Master after finishing my Bachelor degree. I have to thank as well Clemens Padberg, who was always willing to help me with any request I had. Additionally, I would like to express my gratitude to the Sans secretaries Nicole and Izabel.

During my PhD period, I had the opportunity to work with great people on exciting projects. Jens, massive thanks for synthesizing the Azobenzene dendrimer. This molecule was the start for a fruitful collaboration with great scientific discussions. I'm sure your motivation and encouragement will help you to find a good position. I would like to thank Rajesh, for his patience and hard work to perform the microfluidic experiments presented in Chapter 8. Without your endurance, this project would have never been successful. Thank you Mark Smithers for all SEM images provided and the nice chats we had during our countless SEM measurements.

During my PhD, I had the pleasure to supervise a couple of students. Diana, Karabed and Eugenio thanks for your efforts and contributions you have put into my assignments.

I have to thank my paranymphs Wies and Sarah. It's great to have both of you next to me at the end of this journey. Wies, we have had two great years together and became really good friends: Drinking thousand cappuccinos, having countless funny chats, running a few kilometers and had just 3 Tequillas $:$. Our WhatsApp chats are legendary and should never stop. I'm happy that you didn't went to Singapore and wish you so much getting "your" appropriate job soon. Sarah, vielen Dank für die unzähligen Gespräche, die wir geführt haben. Durch deine offene Art hast Du mir oft dabei geholfen, meine eigene Sichtweise zu überprüfen, zu überdenken oder gar zu ändern. Dein Arbeitseifer und dein unbändiges Bedürfnis, den Sachen auf den Grund zu gehen, machen aus Dir die perfekte Wissenschaftlerin. Bleibe immer positiv, denn es wartet etwas ganz wundervolles auf Dich. Auch wenn du manchmal glauben magst, es geht nicht weiter, ich bin mir sicher, Du wirst es schaffen!

A lot of great moments and crazy travels I have had with Raluca. Sharing is caring- that's your way of living. You have been a real friend for me, listening to all my problems and kept motivating me in all situations. I hope you can settle down in Spain for real and we will enjoy many more MUSE moments together.

I would like to thank my Lab 3 mates Raquel, Carlo, Laura, Alejandro, Maarten, Timon, Ina, Gavin, Anna, Janine, Pengkun and Jiguang for all the good moments we had. Raquel, struggling with similar challenges- we have had great times together. Mimi, your Colombian temperament brought another spirit into our group. We could laugh and even cry together. I believe you will as well finish soon and enjoy life with Thomas. Carlo - paparazzi, thanks for your support, synthetic discussions and for keeping the lab organized. I really enjoyed working with you. I wish 
you and Mariska all the best in life... I still have to laugh about the fact, that you are getting married $:-$. Alejandro and Laura, thanks for all the great scientific discussions we had. All the best for your future. Maarten, your humor and German song translation skills are beyond words!

I would like to thank the Dutch community (Pieter, Rick, Jasper, Sven, Roald, Janneke, Wouter, Mark, Mark, Stan, Rik, Rianne and Tom) for all the nice and funny chats we have had. Pieter- ich weiß eigentlich nicht, ob Du hier eingruppiert gehörst. Allein die Tatsache, dass ich jetzt schon wieder Deutsch schreibe, spricht Bände... Wir haben über viele Jahre sehr viel Spaß und manchmal auch Leid miteinander geteilt. Ich wünsche Dir und Aike für die Zukunft vom Herzen alles erdenklich Gute. Rick, thanks for correcting my Dutch summary. I really look up to your positive and relaxed way to see life and the PhD. I hope you will finish soon and have a great time with Alex. Jasper, we have started together and I really enjoyed our funny chats and the time we spent in San Francisco. Svenneke, thanks for the inspiring scientific talks and all the fun we have had together. Roald, thanks for driving us through the north of Spain during our legendary road trip.

I would like to thank all the other people of the MnF and BNT groups that shared with me so many great moments and helped me during my daily lab life: Aijie, Alexander, Andrea, Andreas, Angel, Anne, Ben, Bettina, Chengfeng, Dodo, Erhan, Gülistan, He, Liang, Maike, Martijn, Nickolai, Nicole, Oya, Peter, Piotr, Rindia, Supaporn, Supitchaya, Tushar, Vijay, Xuemei, Yujie. I would like to especially name some people: Emanuela, with whom I really enjoyed working. We have had so many nice talks personally and scientifically. I wish you all the best with Mark and your PhD. Ben, thanks for all the fun you have brought into our labs. We have had countless great moments and conversations. I hope we will visit you (not just your apartment) in Paris quite soon. Jenny and Shirish, you are both such amiable persons struggling as well with the CB chemistry. I wish you all the best for your future and hope your dreams become true. Roberto, after a pretty bad start in Thailand, we did very well in the end. I really admire how you managed to finish your PhD, working with these incredible microreactors. Wilfried, we just shared the office in the last months, but I will always remember you singing at the computer and your face turning red when we told you crazy stories.

There are still a few people from the good old Langezijds period, who definitely have to be named here. Ok, some of them had physically moved to Carre, but for me they are still part of the Langezijds family. Jordi, thank you for the countless funny, happy but as well serious moments. You have been a great Master supervisor. I wish you and Danny all the best, hopefully in the Netherlands. Arancha, we were just living together for few months, never saw each other at home, but managed to become friends. I hope our small families will meet soon. Jealemy and Albert both great colleagues, with a lot of temperament and sometimes too little patience. All the best in living your dreams. Kim, let's call you the master of disaster... We have "shared" our lab items from the beginning, and after a couple of years we could even communicate. Shu-Han, it was such a pleasure to meet you. A little woman discovering and shaping the world. I will never 
forget your wedding in Thailand. Thanks for all the funny moments we have had together. Melanie and Alberto we have had great dialogues... enjoy your life in Austria. And last but not least Muddasir, my dear Mr Pough. After such a bad start (my goodness you told me I'm stupid) we became perfect colleagues with a lot of humor. Your "helicopter" managed to fly always safe and finished her PhD.

Zu guter letzt möchte ich meiner ganzen Familie für den Beistand und Ihre kontinuierliche Unterstützung auf eine ganz besondere Weise danken. Auch wenn Ihr manchmal nicht ganz nachvollziehen konntet, warum ich mich all diesen herausfordernden Strapazen ausgesetzt habe, so habt Ihr immer sehr viel Verständnis für mich gehabt und mich in meinem unbremsbaren Arbeitseifer sogar noch in vielfältiger Weise unterstützt. Ihr sollt wissen, wie unendlich wichtig Ihr für mich ward und seid. Ganz speziell möchte ich natürlich auch Dir, Jan, danken. Ich weiß, daß es für dich nicht der einfachste Moment war, als ich mir nach meinem Master nun noch meine Promotion als Ziel gesetzt habe. Ich erinnere mich noch sehr gut an unser gemeinsames „Entscheidungsgespräch" zurück. Danach hast Du mich aber immer bedingungslos unterstütztmehr noch, ohne Dich wäre es mir unmöglich gewesen, die Reise meines Doktortitels bis zu Ende zu machen. Du hast es immer wieder geschafft mich aufzubauen, besonders an den sehr schwierigen Tagen. Jetzt endlich und nach einer "kleinen aber wertvollen Verzögerung" können wir unser gemeinsames Glück Liesbeth ebenso in den Händen halten, wie auch diese Arbeit. 


\section{Curriculum Vitae}

Carmen Stoffelen was born on the $10^{\text {th }}$ of June 1983, in Kleve, Germany. After finishing her professional education (Chemielaborant) at Bayer in Uerdingen in 2005 she was working as research scientist synthesizing fine chemicals. In 2006 she started studying Chemistry at SaxionUniversity of Applied Sciences, the Netherlands, where she received her bachelor degree in July 2008 (cum laude). During her internship and bachelor project she was working on the development of a durable acid catalyst inside a microfluidic chip. This work was carried out in the Molecular Nanofabrication group at the University of Twente under the supervision of Dr. Wim Verboom and Prof. dr. ir. Jurriaan Huskens.

In August 2010 she finished her master Chemical Engineering at the University of Twente, the Netherlands. During her master project she was working on the supramolecular immobilization of bifunctional peptides. This work was carried out under the supervision of Prof. dr. ir. Pascal Jonkheijm and Prof. dr. ir. Jurriaan Huskens in the Molecular Nanofabrication group at the University of Twente.

Since October 2010, she was Ph.D candidate under the supervision of Prof. dr. ir. Jurriaan Huskens. The aim of the project was the formation of size-tunable supramolecular nanoparticles. The results of the research are described in this thesis. 
